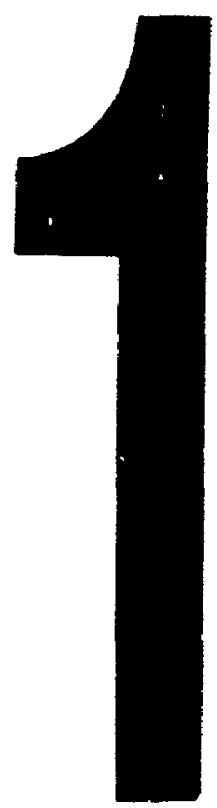

PM-1 3\%/2"x4" PHOTOGRAPHIC MICROCOPY TARGET , NBS 1010a AMSI/ISO \#2 EQUIVALENT

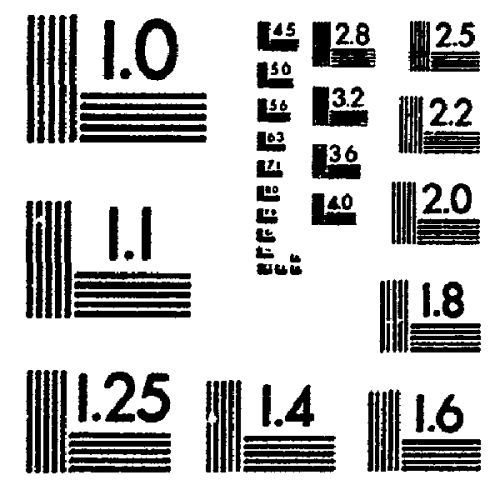


National Library

of Canada

Canadian Theses Service

Ontawa, Canada

KIA ON4
Bibliothèque nationale

du Canada

Service des thèses canadiennes

\section{NOTICE}

The quality of this microform is heavily dependent upor: the quality of the original thesis submitted for microfitming. Every effort has been made to ensure the highest quality of reproduction pussible.

If pages are missing, contact the university which granted the degree.

Some pages may have indistinct print especially if the original pages were typed with a poor typewriter ribbon or if the university sent us an inferior photocopy.

Reproduction in full or in part of this microtorm is; governed by the Canadian Copyright ACl, R.S.C. 1970, C C-30, and su'ssequent amendments.

\begin{abstract}
AVIS
La qualité de cette microforme dépend granderrient de la qualiné de la thèse soumise au microfímage. Nous avons tout fait pour assurer une qualité supérieure de reproduction.

S'il manque des pages, veuillez communiquer avec luniversité qui a conféré le grade.

La qualité d'impression de certaines pages peut laisser à désirer, surtout si les pages originales ont été dactylographiéec a l'aide d'un nuban usé ou si l'université nous a fait parvenir une photocopie de qualité inférieure.
\end{abstract}

La reproduction, méme partielle, de cefte microforme est soumise a la Loi canadienne sur le droit d'auteur, SRC 1970 , c. C.-30, et ses amendements subséquents. 


\title{
Coding and Optimum Baseband Combining for Wideband TDMA Indoor Wireless Channels
}

\author{
by \\ Charles L.B. Despins, B.Eng., M.Eng.
}

\author{
A thesis submitted to \\ the Faculty of Graduate Studies and Research \\ in partial fulfillment of \\ the requirements for the degree of \\ Doctor of Philosophy \\ Ottawa-Carleton Institute for Electrical Engineering \\ Faculty of Engineering \\ Department of Systems and Computer Engineering \\ Carleton University \\ Ottawa, Canada \\ August $20^{\text {th }}, 1991$ \\ (C) copyright \\ 1991, Charles L.B. Despins
}


Canadian Theses Service Service des thèses canadiennes

The author has granted an irrevocable nonexclusive licence allowing the National Library of Canada to reproduce, loan, distribute or sell copies of his/her thesis by any means and in any form or format, making this thesis available to interested persons.

The author retains ownership of the copyright in his/her thesis. Neither the thesis nor substantial extracts from it may be printed or otherwise reproduced without his/her per. mission.
L'auteur a accordé une licence irrévocable et non exclusive permettant à la Bibliothèque nationale du Canada de reproduire, prêter. distribuer ou vendre des copies de sa thèse de quelque manière et sous quelque forme que ce soit pour mettre des exemplaires de cette thèse à la disposition des personnes intéressées.

L'auteir conserve la propriété du droit d'auteur qui protège sa thèse. Ni la thèse ni des extraits substantiels de celle-ci ne doivent être imprimés ou autrement reproduits sans son autorisation. 
The undersigned hereby recommend to the Faculty of Graduate Studies and Research acceptance of the thesis,

"Coding and Optimum Baseband Combining for

Wideband TDMA Indoor Wireless Channels" submitted by

Charles L.B. Despins, B.Eng., M.Eng.

in partial fulfillment of the requirements

for the degree of Doctor of Philosophy

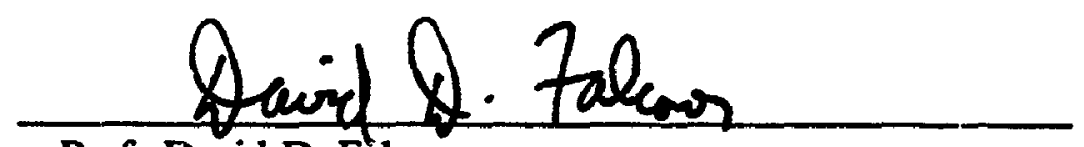

Prof. David D. Falconer

Thesis Co-Supervisor

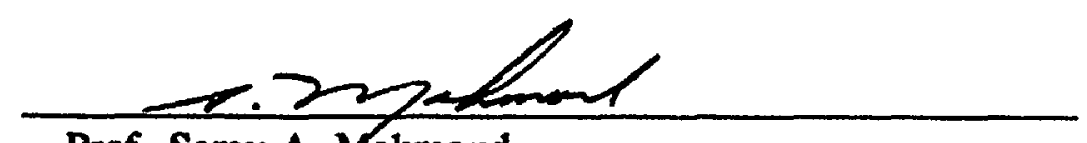

Prof. Samy A. Mahmoud

Thesis Co-Supervisor

Chair, Department of Systems and Computer Engineering

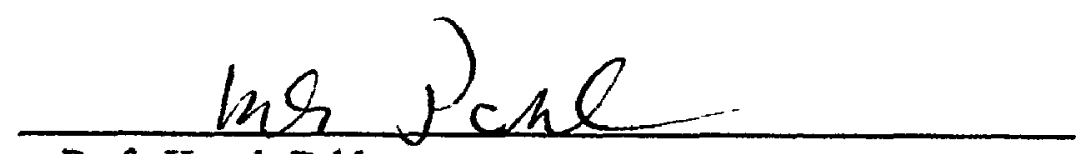

Prof. Kaveh Pahlavan

External Examiner

Worcester Polytechnic Institute, Worcester, Ma., U.S.A.

Carleton University

August $20^{\text {th }}, 1991$ 


\section{Abstract}

A wideband time-division multiple-access (TDMA) access mode is of interest for personal and portable tetherless communications as it provides users with a flexible combination of data rates to accommodate a variety of different applications. Compound strategies of equalization and space diversity in the form of a minimum mean-square error (MMSE) or optimum baseband combiner are attractive candidates to combat dispersive fading and cochannel interference on these radio links. As a space diversity order larger than two may be difficult to realize in a compact form, channel coding may be considered as an alternative to improve perfurmance. This thesis thus presents a comprehensive investigation of cptimum baseband combining in conjunction with convolutional channel coding over Rayleigh fading wideband TDMA indoor radio links perturbed by white noise and cochannel interference.

For this purpose, performance is evaluated using three different approaches. First, a fast analytical approach is employed; it involves the application of previous uncoded bit error rate bounds based on a non-fading gaussian characterization of interference and the development of corresponding novel bounds for the coded case with ideal interleaving. Second, a bit-by-bit simulation is performed in order to evaluate performance without interleaving. Such a simulation is also employed to assess the degree of cyclostationarity of cochannel interference. The third approach is semi-analytical and involves the calculation, via the method of moments, of optimum baseband combiner performance over a static channel and Monte Carlo averaging over the fading distribution. Expressions are developed for the moment-generating function of the decision variate with and without channel coding, and in the former case for both no interleaving and ideal interleaving which is difficult to realize in view of the quasi-static nature of the channel. This technique does not involve a gaussian characterization of interference which is not realistic as one interferer most often dominates. It is shown that as the signaling rate increases, exploiting the channel's implicit diversity effect can make the use of a simple, short constraint length convolutional code a viable altemative to increasing the space diversity order from two to three even the absence of interleaving. 


\section{Résumé}

L'élaboration d'un système de communication universel, personel et portatif suscite un intérêt grandissant pour des liaisons à bande large avec accès multiple à répartition dans le temps $(A M R T)$. De telles liaisons sont avantageuses en ce qu'elles permettent d'offrir aux usagers une combinaison flexible de débits pour la prestation de divers téléservices. Un égaliseur non linéaire muni de plusieurs filtres transversaux et d'un filtre recursif commun, realisant une combinaison optimale ou à erreur quadratique moyenne minimale $(E Q M M)$ en bande de base, s'avère ici un dispositif efficace pour contrer l'évanouissement du signal et l'interférence intersymbole et cocanal qui pertubent les liaisons entre l'abonné mobile et la station de base. Cette thèse présente done une étude détaillée des performances sur de telles liaisons de la combinaison optimale en bande de base associée au codage convolutif que l'on considère en tant qu'altenative à l'adjonction d'une troisième antenne lorsque la conception d'une structure compacte à plus de deux antennes devient problématique.

Cette évaluation comporte trois volets. En premier lieu, une nouvelle borne analytique sur le taux d'erreur du système codé est élaborée à partir d'une caractérisation gaussienne du brouillage et en supposant un entrelacement idéal ce qui est proscrit par les variations très lentes du canal. On évalue ensuite les performances en l'absence d'entrelacement à l'aide d'une símulation. Cette technique sert également à caractériser l'aspect cyclostationaire du brouillage cocanal. Enfin, on utilise un procéde semianalytique qui s'appuie sur la méthode des moments éliminant ainsi la caractérisation gaussienne du brouillage qui s'avère erronnée en raison de la prééminence d'un seul brouilleur. On détermine alors la fonction caractéristique de la variable de décision du système codé non seulement en supposant un entrelacement idéal mais aussi sans entrelacement. On montre que pour des débits suffisamment élevés, l'usage d'un code convolutif avec une courte longueur de contrainte constitue une alternative valable a l'adjonction d'une troisième antenne et ce, grâce à l'association de trajets multiples

par combinaison optimale en bande de base permettant de contrebalancer l'absence d'entrelacement. 


\section{Acknowledgments}

The support and guidance provided by professors David D. Falconer and Samy A. Mahmoud are gratefully acknowledged. Their innumerable suggestions and advice played a great role in bringing this research to fruition. The author would also like to thank current and former fellow graduate students Brent Petersen, Majeed Abdulrahman, Norm Lo, Wilf Leblanc, Steve Todd, Shahram Golestaneh, Dr. Stewart Crozier, Dr. Gary Boudreau as well as research associate Dr. Salim Hanna for many lengthy and enlightening discussions. Thanks are also due to Naren Mehta, Dave Sword and Danny Lemay whose steadfast maintenance work provided the tools necessary for this work and to Vivienne Gilchrist and other secretarial staff for their contribution to a harmonious working environment.

The author also acknowledges the contribution of R.C. Bernhardt of Bell Communications Research in Red Bank, N.J. for cochannel interference statistics provided by his simulations of macroscopic diversity architectures.

This research was supported by grants from the Natural Sciences and Engineering Research Council of Canada (NSERC) and the Telecommunications Research Institute of Ontario (TRIO).

Christine, mille mercis ... 


\section{Table of Contents}

Abstract $\ldots \ldots \ldots \ldots \ldots \ldots \ldots \ldots \ldots \ldots \ldots \ldots \ldots \ldots$ iii

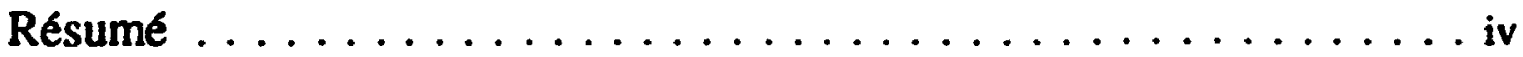

Acknowledgments $\ldots \ldots \ldots \ldots \ldots \ldots \ldots \ldots \ldots \ldots$

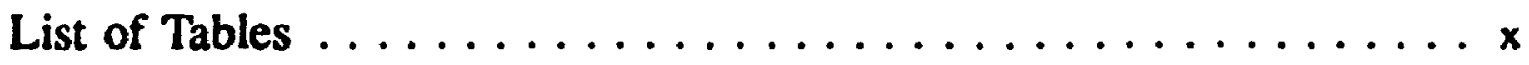

List of Figures $\ldots \ldots \ldots \ldots \ldots \ldots \ldots \ldots \ldots \ldots \ldots$

List of Acronyms and Abbreviations . . . . . . . . . . xx

List of Mathematical Symbols and Notation. . . . . . . . xxiii

Chapter 1 INTRODUCTION . . . . . . . . . . . . . . 1

1.1 Thesis organization $\ldots \ldots \ldots \ldots \ldots \ldots \ldots \ldots \ldots \ldots \ldots \ldots$

1.2 Thesis contributions $\ldots \ldots \ldots \ldots \ldots \ldots \ldots \ldots$

Chapter 2 BACKGROUND and LITERATURE SURVEY . . . . . . 15

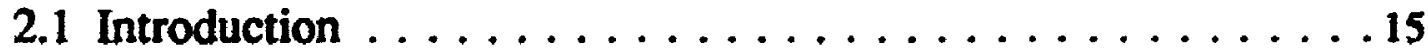

2.2 Characterization of wideband indoor wireless channel . . . . 15

2.2.1 Channel model . . . . . . . . . . . . . 16

2.2.2 Propagation measurements and their influence on channel modeling and system design . . . . . . . . . . . 23

2.2.3 Cochannel interference considerations . . . . . . . 28

2.2.4 Summary of indoor wideband channel characteristics . . 32

2.3 Countermeasure techniques for wideband incioor channel . . . 33

2.3.1 Techniques to combat dispersive fading $\ldots \ldots \ldots . \ldots 33$

2.3.1.1 Diversity. . . . . . . . . . . . . . 33

2.3.1.2 Equalization $\ldots \ldots \ldots \ldots \ldots \ldots$

2.3 .3 Coding . . . . . . . . . . . . . .41

2.3.2 Techniques to combat cochannel interference . . . . . 45 
2.4 Capacity issues $\ldots \ldots \ldots \ldots \ldots \ldots \ldots \ldots \ldots$

2.5 Summary . . . . . . . . . . . . . . . . 49

Chapter 3 ANALYTICAL PERFORMANCE EVALUATION . . . . . .51

3.1 Introduction $\ldots \ldots \ldots \ldots \ldots \ldots \ldots \ldots \ldots \ldots \ldots \ldots \ldots \ldots$

3.2 System description $\ldots \ldots \ldots \ldots \ldots \ldots \ldots \ldots$

3.3 Determination of output SINR . . . . . . . . . 58

3.4 SINR distribution and bit error rate performance $\ldots \ldots \ldots 64$

3.5 Bit error rate bound for coded system . . . . . . . . . .69

3.6 Summary of analytical assumptions $\ldots \ldots \ldots \ldots \ldots \ldots$

3.7 Results . . . . . . . . . . . . . . . . 74

3.7.1 Principle of optimum combining $\ldots \ldots \ldots \ldots 75$

3.7.2 Equalizer sampling phase $\ldots \ldots \ldots \ldots \ldots$

3.7.3 Number of forward filter taps . . . . . . . . . . 79

3.7.4 Delay spread (signaling rate) $\ldots \ldots \ldots \ldots \ldots \ldots$

3.7.5 Signal-to-interference ratio $\ldots \ldots \ldots \ldots \ldots \ldots$. . . 81

3.7.6 Coding vs. space diversity $\ldots \ldots \ldots \ldots \ldots \ldots$. . . 81

3.7.7 QPSK vs. BPSK . . . . . . . . . . . . . 82

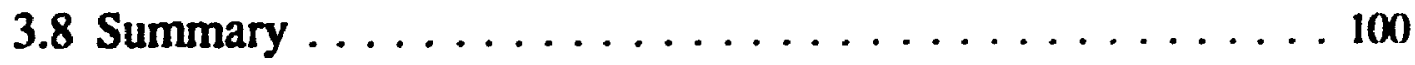

Chapter 4 PERFORMANCE EVALUATION VIA SIMULATION . . . 102

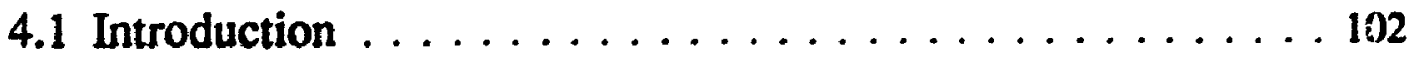

4.2 Channel model . . . . . . . . . . . . . . 103

4.3 Static channel approximation . . . . . . . . . . 106

4.4 Results $\ldots \ldots \ldots \ldots \ldots \ldots \ldots \ldots \ldots \ldots \ldots \ldots$

4.4.1 Cyclostationary nature of interference $\ldots \ldots \ldots \ldots 111$

4.4.2 Average BER and outage performance . . . . . 112

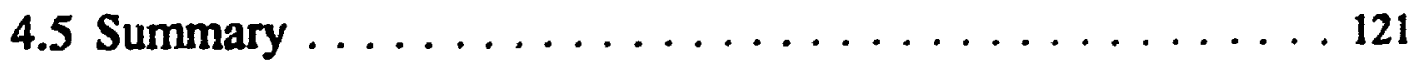


Chapter 5 SEMI-ANALYTICAL PERFORMANCE EVALUATION . 122

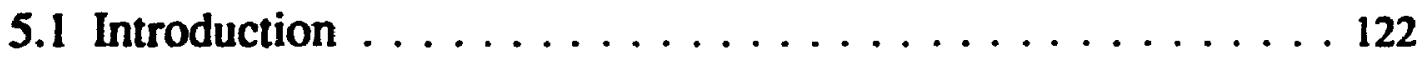

5.2 Description of technique . . . . . . . . . . 122

5.2.1 Uncoded BER . . . . . . . . . . . . . . . 125

5.2.2 Coded BER . . . . . . . . . . . . . . . . . 129

5.2.2.1 Ideal interleaving (random errors) . . . . . . 130

5.2.2.2 No interleaving (burst-error case) . . . . . . 132

5.3 Summary of analytical assumptions $\ldots \ldots \ldots \ldots \ldots$

5.4 Results . . . . . . . . . . . . . . . . . . . 148

5.4 .1 Coding vs. space diversity $\ldots \ldots \ldots \ldots \ldots \ldots \ldots$

5.4.1.1 Effect of cochannel interference . . . . . . . 157

5.4.1.2 Effect of a lower data rate . . . . . . . . 161

5.4.2 Channel model . . . . . . . . . . . . . 163

5.4 .3 Interference model . . . . . . . . . . . . . . 164

5.4 .4 Sampling phase $\ldots \ldots \ldots \ldots \ldots 7$

5.5 Summary . . . . . . . . . . . . . . 200

Chapter 6 CONCLUSION . . . . . . . . . . . . . . . . 202

6.1 Thesis summary $\ldots \ldots \ldots \ldots \ldots \ldots \ldots \ldots \ldots \ldots$

6.1.1 Analytical approach . . . . . . . . . . . 203

6.1.2 Bit-by-bit simulation . . . . . . . . . . . 204

6.1.3 Semi-analytical approach . . . . . . . . . . . 204

6.2 Recommendations for further research . . . . . . . . 207

6.2.1 Influence of diversity vs. coding trade-off on cochannel reuse ratio . . . . . . . . . . . . . . . . . . 207

6.2.2 Performance evaluation with partial interleaving $\ldots 207$

6.2.3 More powerful codes and simplified decoding algorithms . . . . . . . . . . . . . . . 208 
6.2.4 Trellis coding . . . . . . . . . . . . . . 208

6.2.5 Evaluations based on refined channel models . . . . . . 209

6.2.6 Capacity of indoor radio links . . . . . . . . . . 209

Bibliography

Appendix A Recursive computation of partial fraction expansion coefficients . . . . . . . . . . . . 226

Appendix B Bit error rate bounds in flat Rayleigh fading . . . . . 228

Appendix C Simulation documentation $\ldots \ldots \ldots \ldots \ldots \ldots \ldots$

Appendix D Capacity of indoor radio links

236

Appendix E Lagrange multipliers and the calculus of variations . . 241

ix 


\section{List of Tables}

Table 3.1

Sampling phase sensitivities in terms of $\frac{E_{b}}{N_{0}}$ required for $10^{-3}$ average BER. These results correspond to

Figures $3.4,3.5$ and $3.6 \ldots \ldots \ldots 8$

Table 4.1

Simulation results for $N=1, S /=10 \mathrm{~dB}, t_{1}=0.0 \mathrm{~T}$ a) Number of channel sets (out of 20 ) for which average BER $\geq 10^{-3}$ vs. Eb/NO. $t_{0}=0.375 T$. b) Number of channel sets (out of 20) for which average BER $\geq$ $10^{-3}$ vs. $E b / N O$. $t_{0}=0.0 \mathrm{~T}$. c) Average BER for 2 different sampling phases at $\mathrm{Eb} / \mathrm{NO}=70 \mathrm{~dB} . \ldots . .120$ 


\section{List of Figures}

Figure 2.1

Figure 3.1

Figure 3.2

Figure 3.3

Figure 3.4

Figure 3.5

Figure 3.6

Figure 3.7
Distribution of ratio of dominant interferer power (Id) to total interference power (Itot). Data obtained from Bernhardt from a simulation of a frequency reuse system with 4-branch macroscopic diversity, 20 active cochannel cells, path loss exponent $n=4$ and log-normal standard deviation $\xi=10 \mathrm{~dB} . \ldots \ldots \ldots$ i

System model (low-pass equivalent). . . . . . . . 53

MMSE decision feedback equalizer forward and feedback filters. . . . . . . . . . . . . . . . 57

BPSK BER vs. SINR $\Gamma$. Interferer-to-noise ratio $E_{S n} / N 0=1$ for $n=1,2, t_{0}=0.0 T, t_{1}=0.125 \mathrm{~T}, t_{2}=0.25 T$, $\sigma_{\mathrm{d}}=0.25 \mathrm{~T}$, rectangular pulse shape, $\mathrm{L}=3, \mathrm{Ll}=2$, L2 $=0$. 84

E.fect of fixed sampling phase to. $M=1, N=0, L=3$, $\mathrm{L} 1=2, \mathrm{~L} 2=0, \sigma_{\mathrm{d}}=0.25 \mathrm{~T}, \mathrm{BPSK} . \ldots \ldots . \ldots .85$

Effect of fixed sampling phase $t_{0} . M=2, L=3, L 1=2$, L2 $=0, \sigma_{d}=0.25 T$, BPSK. a) $\mathrm{N}=0$ b) $\mathrm{N}=1, \mathrm{~S} / \mathrm{l}=10 \mathrm{~dB}$,

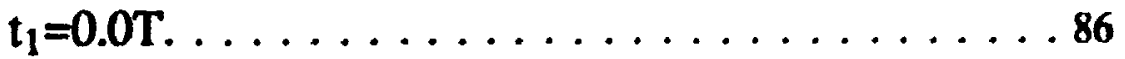

Effect of fixed sampling phase $t_{0} . M=3, L=3, L 1=2$, L2 $=0, \sigma_{d}=0.25 T$, BPSK. a) $\mathrm{N}=0$ b) $\mathrm{N}=1, \mathrm{~S} / \mathrm{I}=10 \mathrm{~dB}$, $\mathrm{t}_{1}=0.0 \mathrm{~T}$.

Effect of number of forward filter taps $L . M=2, N=1$, $\mathrm{L} 1=\mathrm{L}-1, \mathrm{~L} 2=0, \mathrm{t}_{0}=0.375^{\prime} \mathrm{T}, \mathrm{t}_{1}=0.0 \mathrm{~T}, \sigma_{\mathrm{d}}=0.25 \mathrm{~T}, \mathrm{BPSK}$.

a) Time-rectangular pulse b) $50 \%$ SRC pulse. . . . 89 
Figure 3.8

Figure 3.9

Figure 3.10

Figure 3.11

Figure 3.12

Figure 3.13

Figure 3.14

Figure 3.15

Effect of splitting forward filter taps into L1 precursor taps and L2 postcursor taps. $M=2, N=1, t_{0}=0.375 T$, $\mathrm{t}_{1}=0.0 \mathrm{~T}, \sigma_{\mathrm{d}}=0.25 \mathrm{~T}, 50 \%$ SRC pulse, BPSK. . . . 90

Effect of varying rms delay spread $\sigma_{\mathrm{d}} . \mathrm{M}=2, \mathrm{~N}=1$, $\mathrm{S} / \mathrm{I}=10 \mathrm{~dB}, \mathrm{t}_{1}=0.0 \mathrm{~T}$, time-rectangular pulse, $\mathrm{L}=3$, $\mathrm{L} 1=\mathrm{L}-1, \mathrm{~L} 2=0, \mathrm{t}_{0}=0.375 \mathrm{~T}$, BPSK. . . . . . . 91

Effect of varying rms delay spread $\sigma_{\mathrm{d}} . \mathrm{M}=3, \mathrm{~N}=1$, $S / I=10 \mathrm{~dB}, t_{1}=0.0 \mathrm{~T}$, time-rectangular pulse, $\mathrm{L}=3$, $\mathrm{L} 1=\mathrm{L}-1, \mathrm{~L} 2=0, \mathrm{t}_{0}=0.375 \mathrm{~T}$, BPSK. . . . . . . 92

Effect of varying $S / I$ ratio. $N=1, t_{1}=0.0 T, \sigma_{d}=0.25 T$, time-rectangular pulse, $\mathrm{L}=3, \mathrm{~L} 1=\mathrm{L}-1, \mathrm{~L} 2=0$, $t_{0}=0.375 T$, BPSK. a) $M=2$ b) $M=3$.

Effect of varying $S / I$ ratio for coded system. $M=2$, $\mathrm{N}=1, \mathrm{t}_{1}=0.0 \mathrm{~T}, \sigma_{\mathrm{d}}=0.25 \mathrm{~T}$, time-rectangular pulse, $\mathrm{L}=3$, $\mathrm{L} 1=\mathrm{L}-1, \mathrm{~L} 2=0, \mathrm{t}_{0}=0.375 \mathrm{~T}$, BPSK. . . . . . 94

Coding vs. space diversity. $\mathrm{N}=1, \mathrm{t}_{1}=0.0 \mathrm{~T}, \sigma_{\mathrm{d}}=0.25 \mathrm{~T}$, time-rectangular pulse, $L=3, L 1=L-1, L 2=0$, $t_{0}=0.375 T$. 95

Effect of varying rms delay spread $\sigma_{\mathrm{d}} . \mathrm{M}=2, \mathrm{~N}=1$, $S / I=10 \mathrm{~dB}, \mathrm{t}_{1}=0.0 \mathrm{~T}$, time-rectangular pulse, $\mathrm{L}=3$, $\mathrm{L} 1=\mathrm{L}-1, \mathrm{~L} 2=0, \mathrm{t}_{0}=0.375 \mathrm{~T}$, QPSK. . . . . . 96

Effect of varying rms delay spread $\sigma_{d} . M=3, N=1$, $S / I=10 \mathrm{~dB}, t_{1}=0.0 \mathrm{~T}$, time-rectangular pulse, $L=3$, $\mathrm{L} 1=\mathrm{L}-1, \mathrm{~L} 2=0, \mathrm{t}_{0}=0.375 \mathrm{~T}$, QPSK. . . . . . . 97 
Figure 3.16

QPSK vs. BPSK. a) $M=2$ b) $M=3$. $N=1, S / 1=10 \mathrm{~dB}$, $\mathrm{t}_{1}=0.0 \mathrm{~T}, \mathrm{~L}=3, \mathrm{~L} 1=\mathrm{L}-1, \mathrm{~L} 2=0, \mathrm{t}_{0}=0.375 \mathrm{~T}$. QPSK uses $100 \%$ SRC pulse; BPSK uses $0 \%$ SRC pulse. . . . . 98

Figure 3.17

QPSK vs. BPSK. a) $M=2$ b) $M=3 . N=0, L=3, L l=L-1$, $\mathrm{L} 2=0, \mathrm{t}_{0}=0.375 \mathrm{~T}$. QPSK uses $100 \%$ SRC pulse; BPSK uses $0 \%$ SRC pulse. . . . . . . . . . . . . 99

Figure 4.1

Exponential delay profile $\phi(t)$ (dotted line) with rms delay spread equal to one symbol period $\mathrm{T}$. The bar chart (solid line) shows the path variance approximation (normalized to half the delay spread) as specified by equation $4.4 \ldots \ldots \ldots \ldots$. . . . . 105

Figure 4.2

Mean of complex magnitude squared channel output (sum of complex magnitude squared of the 9 complex channel tap coefficients for a given portable to base-station-antenna path) versus number of channel realizations.

Figure 4.3

Distribution of channel output magnitude. a) Probability density function for 100 and 10000 realizations. b) Histogram for 20 realizations. . . . 110

Figure 4.4

Average power (normalized to unity) of the sum of two independent in-phase BPSK signals passed through five different pairs of independent static dispersive Rayleigh fading channel realizations. . . 115

Figure 4.5

BPSK average $B E R$ vs. $E b / N O . ~ M=2, N=0$, $t_{0}=0.0 \mathrm{~T} \ldots \ldots \ldots \ldots \ldots \ldots \ldots \ldots \ldots$ 
Figure 4.6

BPSK average BER vs. SINR for $\mathrm{t} 0=0.0 \mathrm{~T}$. a) analysis vs. simulation for $M=2, N=1, E s 1 / N 0=1, t 1=0.125 T$. b) simulation results for $\mathrm{N}=0$ and $\mathrm{N}=1$ (Es1/NO=1, $\mathrm{t} 1=0.125 \mathrm{~T}) \ldots \ldots \ldots \ldots \ldots \ldots \ldots$

Figure 4.7 Analysis vs. simulation for BPSK average BER vs. Eb/N0. $N=1, S / I=10 \mathrm{~dB}, t 1=0.0 \mathrm{~T}$. a) $\mathrm{t}_{0}=0.375 \mathrm{~T}$. b) $t_{0}=0.0 \mathrm{~T} . \ldots \ldots \ldots \ldots \ldots \ldots \ldots \ldots \ldots \ldots \ldots \ldots$

Figure 4.8 Simulation results for average BER vs. Eb/NO, $N=1$, $\mathrm{S} / \mathrm{I}=10 \mathrm{~dB}, \mathrm{t} 1=0.0 \mathrm{~T}$. a) $\mathrm{t}_{0}=0.375 \mathrm{~T}$. b) $\mathrm{t}_{0}=0.0 \mathrm{~T}$. . . 119

Figure 5.1 Probability distribution function of rms delay spread over 10000 impulse response realizations for two different mean cluster interarrival times. $(1 / \lambda)=5 \mathrm{~ns}$, $\Gamma=60$ ns, $\gamma=20$ ns. $\ldots \ldots \ldots \ldots \ldots \ldots$

Figure 5.2

Test cases with BPSK, $\mathrm{N}=0, \mathrm{~L}=1$, time-rectangular pulse shape and flat Rayleigh fading $\left(\sigma_{\mathrm{d}}=10^{-6} \mathrm{~T}\right.$ with 9-tap channel model). $\mathbf{M}=$ \# of receiver antennas. Coded curves with ideal interleaving. . . . . . . 171

Figure 5.3

Test cases with $M=1, N=0, L=1$, time-rectangular pulse shape and flat Rayleigh fading $\left(\sigma_{d}=10^{-6} \mathrm{~T}\right.$ with 9-tap channel model). Hanna et al. results from a) Average BER b) Outage rate . . . . . . . . 172

Figure 5.4

Test cases with $M=1, N=0, L=1, S R C$ (50\%) pulse shape and flat Rayleigh fading $\left(\sigma_{\mathrm{d}}=10^{-6} \mathrm{~T}\right.$ with 9-tap channel model). a) Average BER b) Outage rate . 173 
Figure 5.5

Average BER vs. outage rate at same value of $\mathrm{Eb} / \mathrm{N} 0$. $N=0, S R C(50 \%)$ pulse shape. a) $M=1, L=1$, flat fading $b$ ) $M=2, L=3$, cluster channel model ( 10 Mbits/s data rate). . . . . . . . . . . . . . . 174

Figure 5.6

Results for no cochannel interference $(\mathrm{N}=0) . \mathrm{L}=3$, cluster channel model (10 Mbits/s data rate), SRC (50\%) puise shape. a) Average BER b) Outage rate 175

Figure 5.7

Results for $\mathrm{N}=1$ interferer. $S I R=10 \mathrm{~dB}, \mathrm{~L}=3$, cluster channel model (10 Mbits/s data rate), SRC (50\%) pulse shape. a) Average BER b) Outage rate . . . . 176

Figure 5.8

Results for $\mathrm{N}=1$ interferer. $E b / N 0=30 \mathrm{~dB}, \mathrm{~L}=3$, cluster channel model (10 Mbits/s data rate), SRC (50\%) pulse shape. . . . . . . . . . . . . . 177

Figure 5.9

Results for $\mathrm{N}=2$ equal-power interferers. $\mathrm{SIR}_{\mathrm{tot}}=10 \mathrm{~dB}$, $L=3$, cluster channel model ( $10 \mathrm{Mbits} / \mathrm{s}$ data rate), $S R C$ (50\%) pulse shape. a) Average BER b) Outage rate 178

Figure 5.10

Results for $\mathrm{N}=2$ equal-power interferers. $\mathrm{Eb} / \mathrm{N} 0=30 \mathrm{~dB}$, $L=3$, cluster channel model (10 Mbits/s data rate), SRC (50\%) pulse shape. a) Average BER b) Outage rate 179

Figure 5.11

Results for $\mathbf{N}=2$ interferers with different power distributions. $\operatorname{SIR}_{\mathrm{tot}}=10 \mathrm{~dB}, M=2, L=3$, cluster channel model (10 Mbits/s data rate), SRC (50\%) pulse shape. Coded results with no interleaving. a) Average BER b) Outage rate . . . . . . . . . . . . . 180 
Figure 5.12

Results for $\mathrm{N}=\mathbf{2}$ interferers with different power distributions. SIR tot $=10 \mathrm{~dB}, M=2, L=3$, cluster channel model (10 Mbits/s data rate), SRC (50\%) pulse shape. Coded results with no interleaving. a) Average BER b) Outage rate

Figure 5.13

Results for $\mathbf{N}=\mathbf{2}$ interferers with different power distributions. $E b / N 0=30 \mathrm{~dB}, \mathrm{M}=2, \mathrm{~L}=3$, cluster channel model (10 Mbits/s data rate), SRC (50\%) pulse shape. Coded results with no interleaving. a) Average BER b) Outage rate . . . . . . . . . 182

Figure 5.14

Results for $\mathbf{N}=\mathbf{2}$ interferers with different power distributions. $E b / N 0=30 \mathrm{~dB}, M=2, L=3$, cluster channel model (10 Mbits/s data rate), SRC (50\%) pulse shape. Coded results with no interleaving. a) Average BER b) Outage rate . . . . . . . . . . 183

Figure 5.15

Results for $N=1$ interferer. $L=3$, cluster channel model (10 Mbits/s data rate), SRC (50\%) pulse shape. a) Average BER b) Outage rate . . . . . . . . . . . 184

Figure 5.16

Results for $\mathrm{N}=1$ interferer. $E b / N 0=30 \mathrm{~dB}, \mathrm{~L}=3$, cluster channel model (10 Mbits/s data rate), SRC (50\%) pulse shape. Coded QPSK curve is the same as in Figure 5.8 but shifted by $6 \mathrm{~dB}$ to account for bandwidth expansion. . . . . . . . . . . . 185

Figure 5.17

BPSK vs. QPSK with $\mathbf{N}=0$ interferer. $\mathrm{L}=3$, cluster channel model (10 Mbits/s data rate), SRC (50\%) pulse shape. a) Average BER b) Outage rate . . . 186 
Figure 5.18

Figure 5.19

Figure 5.20

Figure 5.21

Figure 5.22

Figure 5.23

Figure 5.24
BPSK vs. QPSK with $N=1$ interfeter. $E b / N 0=30 \mathrm{~dB}$, $\mathrm{L}=3$, cluster channel model (10 Mbits/s data rate), SRC (50\%) pulse shape. . . . . . . . . . . . 187

Results at a lower data rate with $\mathrm{L}=3$ forward filter taps. $M=2, N=1$, cluster channel model (1 Mbits/s), SRC (50\%) pulse shape. a) Average BER b) Outage rate 188

Results at a lower data rate with $\mathrm{L}=1$ forward filter tap. $S I R=10 \mathrm{~dB}, M=2, N=1$, cluster channel model ( 1 Mbits/s), SRC (50\%) pulse shape. a) Average BER b) Outage rate . . . . . . . . . . . . . 189

Results at a lower data rate with $L=1$ and $L=3$ forward filter taps. $E b / N O=30 \mathrm{~dB}, M=2, N=1$, cluster channel model (1 Mbits/s), SRC (50\%) pulse shape. . . . . 190

Results for different channel models. Equal-power interferers $(N=2), S_{\text {tot }}=10 \mathrm{~dB}, M=2, L=3, \sigma_{d}=0.45 T$, BPSK, SRC (50\%) pulse shape. a) Average BER b) Outage rate . . . . . . . . . . . . . . 191

Results for different channel models. Equal-power interferers $(\mathrm{N}=2), \mathrm{Eb} / \mathrm{NO}=30 \mathrm{~dB}, \mathrm{M}=2, \mathrm{~L}=3$, $\sigma_{\mathrm{d}}=0.45 \mathrm{~T}, \mathrm{BPSK}, \mathrm{SRC}(50 \%)$ pulse shape. 192

Method of moments vs. analysis. $M=2, N=0, L=3$, $\sigma_{\mathrm{d}}=0.45 \mathrm{~T}, \mathrm{BPSK}, \mathrm{SRC}$ (50\%) pulse shape, 9-tap channel model for method of moments. a) Average BER b) SINR P.D.F.'s at Eb/N0=30dB. . . . . . . 193 
Figure 5.25

Method of moments vs. analysis. $M=2, N=1, S I R=10$ $\mathrm{dB}, \mathrm{L}=3, \sigma_{\mathrm{d}}=0.45 \mathrm{~T}, \mathrm{BPSK}, \mathrm{SRC}(50 \%)$ pulse shape, 9-tap channel model for method of moments. a) Average BER b) SINR P.D.F.'s at Eb/NO=30dB. . 194

Figure 5.26

Method of moments vs. analysis. $M=2, N=2, S I R_{t o t}=10$ $\mathrm{dB}, \mathrm{L}=3, \sigma_{\mathrm{d}}=0.45 \mathrm{~T}, \mathrm{BPSK}, \mathrm{SRC}(50 \%)$ pulse shape, 9-tap channel model for method of moments. a) Average BER b) SINR P.D.F.'s at Eb/NO=30dB. . 195

Figure 5.27

Fixed vs. continuously optimized sampling phase. $M=1, N=0, L=3, B P S K, 9-t a p$ channel model, $\sigma_{\mathrm{d}}=0.25 \mathrm{~T}$, time-rectangular pulse shape. a) Average BER b) Outage rate . . . . . . . . . . . 196

Figure 5.28

Fixed vs. continuously optimized sampling phase. $\mathrm{M}=1, \mathrm{~N}=0, \mathrm{~L}=3$, BPSK, 9-tap channel model, $\sigma_{d}=0.50 \mathrm{~T}$, time-rectangular pulse shape. a) Average BER b) Outage rate . . . . . . . . . . . . . . . . 197

Figure 5.29

Fixed vs. continuously optimized sampling phase. $M=1, N=0, L=3, B P S K, 9$-tap channel model, $\sigma_{d}=0.25 T$, SP.C (50\%) pulse shape. a) Average BER b) Outage rate . . . . . . . . . . . . . . . 198

Figure 5.30 Fixed vs. continuously optimized sampling phase. $\mathrm{M}=1, \mathrm{~N}=0, \mathrm{~L}=3$, BPSK, 9-tap channel model, $\sigma_{d}=0.50 \mathrm{~T}, \mathrm{SRC}(50 \%)$ pulse shape. a) Average BER b) Outage rate . . . . . . . . . . . . . . . . . . . 199 
Figure B.1

Theoretical BER bounds from [71]. $N=0, L=1$, fiat Rayleigh fading, time-rectangular pulse shape. a) Uncoded, $\mathbf{M}=$ \# of antennas $\mathbf{b}$ ) Coded with ideal interleaving, $M=1$ (rate $1 / 2$ convolutional code, $\mathrm{d}_{\text {free }}=5$, constraint length 3 ). . . . . . . 230

Figure C.1 BOSS module for the two receiver antenna system with coding. . . . . . . . . . . . 232

Figure C.2 BOSS module for the (coded) transmitter and channel. Corresponds to the "M=2_CODED Tx \& CHANNEL (STATIC)" module in Figure C.1. . . . . . . . 233

Figure C.3 Example of a parameter set for the system in Figure C.1. . . . . . . . . . . . . . . . . . 234

Figure C.4 Example of a results set for the system in Figure C.1. . . . . . . . . . . . . . . 235

Figure D.1 Multi-input, multi-output system model for $(\mathrm{N}+1)$ portables and a base station with $\mathbf{M}$ antennas. Channel matrix is $\mathrm{M} \times(\mathrm{N}+1)$ while transmitter matrix is $(\mathrm{N}+1) \times(\mathrm{N}+1)$. . 


\section{List of Acronyms and Abbreviations}

AGC - automatic gain control

AMPS - Advanced Mobile Phone Service

AWGN - additive white gaussian noise

BCH - Bose Chaudhuri Hocquenghem

BER - bit error rate

BOSS - Block Oriented Systems Simulator ${ }^{\mathrm{m}}$

BPSK - binary phase-shift keying

CB - citizens' band

CCI - cochannel interference

CDMA - code division multiple access

CRI - cross-rail interference

$\mathrm{CW}$ - continuous wave

dB - decibel

DECT - Digital European Cordless Telephone

DFE - decision feedback equalizer

DPSK - differential phase-shift keying

FDMA - frequency division multiple access

FSE - fractionally spaced equalizer

FSK - frequency-shift keying

$\mathrm{GHz}$ - gigahertz $=10^{9} \mathrm{~Hz}$

GMSK - gaussian minimum shift keying

GQR - gaussian quadrature algorithm

GSM - Groupe Spécial Mobile

GWSSUS - gaussian wide-sense stationary uncorrelated scattering

HCMTS - High Capacity Mobile Telephone Service

HF - high frequency

$\mathrm{Hz}$ - hertz (cycles per second) 
IF - intermediate frequency

ISDN - Integrated Services Digital Network

ISI - intersymbol interference

Kbits/s $-10^{3}$ bits per second

Ksymbols/s $-10^{3}$ symbols per second

$\mathrm{KHz}$ - kilohertz $=10^{3} \mathrm{~Hz}$

$\mathrm{km} / \mathrm{hr}$ - kilometers per hour

LMS - least mean square

Mbits/s - $10^{6}$ bits per second

mgf - moment-generating function

$\mathrm{MHz}$ - megahertz $=10^{6}$ hertz

Msymbols/s - $10^{6}$ symbols per second

MLSE - maximum likelihood sequence estimation

MMSE - minimum mean squared error

MSE - mean squared error

MSK - minimum shift keying

MPSK - M-ary phase-shift keying

NMT - Nordic Mobile Telephone

$\mathrm{ms}$ - milliseconds $=10^{-3}$ seconds

ns - nanoseconds $=10^{-9}$ seconds

PAM - pulse amplitude modulation

PBX - private branch exchange

pdf - probability density function

PCN - Personal Communications Network

QAM - quadrature amplitude modulation

QPSK - quadrature phase-shift keying

RF - radio frequency

RLS - recursive least squares

RMS - root mean square 
RS - Reed-Solomon

$\mathbf{R x}$ - receiver

sec - second

SHF - super high frequency

SINR - signal to interference plus noise ratio

SIR - signal to interference ratio

$S_{\text {IRt }}$ - signal to total interference ratio

SNR - signal to noise ratio

SRC - spectral raised cosine

TACS - Total Access Communications System

TDMA - time division multiple access

TFM - Tamed frequency modulation

TIA - Telecommunications Industry Association

Tx - transmitter

TV - television

UDPC - Universal Digital Portable Communications

UHF - ultra high frequency

$\mu \mathrm{s}-$ microsecond $=10^{-6}$ second

U.S. - United States (of America)

VA - Viterbi algorithm

VHF - very high frequency

VLSI - very large scale integration 


\section{List of Mathematical Symbols and Notation}

A - general matrix

$A^{*}$ - complex conjugate of $A$

$A^{-1}$ - matrix inverse of $A$

$\mathbf{A}^{\mathbf{T}}$ - transpose of $\mathbf{A}$

$A_{i j}$ - partial fraction expansion coefficients

$a_{k n}-$ in-phase $k^{\text {th }}$ transmitted bit from $n^{\text {th }}$ portable

$B$ - number of feedback filter taps

$B_{c}$ - coherence bandwidth

$B_{d}$ - Doppler spread

$b_{k n}$ - quadrature $k^{\text {th }}$ transmitted bit from $n^{\text {th }}$ portable

C - channel capacity (bits/sec)

$\mathrm{C}_{\mathrm{W}}$ - normalized channel capacity (bits/sec/Hz)

$C-(M \times L) \times(M \times L)$ interference plus noise short-term covariance matrix

$C_{0}-(M \times L) \times(M \times L)$ additive white gaussian noise short-term covariance matrix

$C_{1}-(M \times L) \times(M \times L)$ interference short-term covariance matrix

$\cosh ($.$) - hyperbolic cosine of term$

$d_{\text {free }}-$ minimum free distance of code

$\mathbf{E}[$.$] - short-term average$

$E_{b}$ - energy per bit

$E_{s}$ - energy per symbol (from desired signal)

$E_{5 n}$ - energy per symbol from $n^{\text {th }}$ cochannel interferer

$\operatorname{erfc}($.$) - complementary error function$

$\operatorname{exp(.)}$ - natural exponent function

$f_{c}$ - carrier frequency

$f(t)$ - transmit/receive pulse shaping function 
$G_{X}(s)$ - Laplace transform of probability densiiy function of $\mathbf{X}$

$g(s)$ - moment-generating function of decision variable

$g_{\eta}(s)$ - moment-generating function of interference component of decision variable

$\mathrm{g}_{\xi}(\mathrm{s})$ - moment-generating function of AWGN component of decision variable

$\mathbf{g}_{\lambda}(s)$ - moment-generating function of desired signal component of decision variable

$g(t)$ - transmitter pulse shape

$g(-t)$ - receiver pulse shape

$h(t ; \tau)$ - low-pass equivalent time-variant channel response at time $t$ to an impulse applied at $\mathbf{t}-\tau$.

$H(f ; t)$ - Fourier transform of $h(t ; \tau)$

I - identity matrix

$I_{i}()-.i^{i^{\text {th }}}$-order modified Bessel function of the first kind

Im(.) - imaginary part of complex number

$I_{n}-$ mean power from $n^{\text {th }}$ cochannel interferer

$j$ - square root of negative one; also an integer index

$K$ - significant duration (in symbol intervals $T$ ) of pulse function $q(t)$

$L$ - number of forward filter taps

$\mathrm{Ll}$ - number of forward filter precursor taps

L2 - number of forward filter postcursor taps

$\ln ($.$) - natural logarithm$

$\log ($.$) - base 10$ logarithm

$M$ - number of receiver antennas

n - propagation power law exponent

$n_{i}$ - multiplicity of $i^{\text {th }}$ eigenvalue

$n_{i}(t)$ - complex additive white gaussian noise process at $i^{\text {th }}$ receiver antenna $\mathrm{N}$ - number of cochannel interferers 
$\mathrm{N}_{0}$ - single-sided noise power spectral density

$\mathrm{N}_{\mathrm{p}}$ - mean received noise power

$P_{2}(d)$ - pairwise error probability fol sequences that differ in $d$ bits

$P_{b}$ - bit error probability

$P_{s}$ - symbol error probability

$\mathrm{q}_{\mathrm{ni}}(\mathrm{t})$ - convolution of transmit/receive pulse shape and impulse response between $\mathrm{n}^{\text {th }}$ portable and $\mathrm{i}^{\text {th }}$ receiver antenna

$q_{k n}-(M \times L)$-element combined sampled filter/channel response vector from $n^{\text {th }}$ portable at $t_{0}-k T$

$\mathbf{R}$ - short-term desired symbol covariance matrix

$R_{\mathrm{c}}$ - code rate (in information bits per coded bits)

$\operatorname{Re}($.$) - real part of complex number$

$r_{i}(t)$ - input to $i^{\text {th }}$ forward filter

$S$ - mean power from desired portable

S/H - sample and hold

$S_{H}($.$) - Doppler power spectrum$

$s_{k n}-k^{\text {th }}$ transmitted symbol fron $n^{\text {th }}$ portable

so - saddlepoint search starting point

sech(.) - hyperbolic secant of term

$\operatorname{sum}(A)$ - sum of elements of vector A

$t$ - continuous time variable

$\mathrm{T}$ - symbol interval

$T_{c}$ - coherence time

$T_{1}$ - arrival time of $t^{\text {th }}$ cluster

$t_{0}$ - receiver sampling phase

$t_{n}-n^{\text {th }}$ interferer time shift with respect to desired signal

tanh(.) - hyperbolic tangent of term

$U^{(r)}-$ Viterbi decoder metric at $r^{\text {th }}$ node 
$V-(M \times L)$-element sampled additive white gaussian noise vector

$v$ - velocity

var(.) variance of term

W - bandwidth

$W-(M \times L)$-element vector of forward filter tap weights

$W_{\text {opt }}-(M \times L)$-element vector of forward filter tap weights minimizing mean-square error

$w_{i l}-1^{\text {th }}$ tap in $i^{\text {th }}$ forward filter

$x_{b}-$ sum of forward filter outputs sampled at $t_{0}+b T$

$z$ - input to decision device

$\alpha(t ; \tau)$ - continuous time multipath response attenuation function

$\alpha_{\mathrm{r}}$ - magnitude of $\mathrm{r}^{\text {th }}$ complex channel tap coefficient

$\beta_{d}-$ coefícients of first derivative of code transfer function

$\beta_{\mathbf{k l}}-$ gain of $\mathbf{k}^{\text {th }}$ ray within $\mathbf{l}^{\text {th }}$ cluster

$x$ - Doppler frequency variable

di $(i)$ - Dirac, delta or unit impulse function

$\eta$ - interference component of decision variable

$\Gamma$ - signal to interference plus noise ratio; also used for cluster power-delay time constant

$\gamma$ - standard deviation of gaussian interfering symbols; also used for ray power-delay time constant

$\lambda$ - wavelength; also used for ray arrival rate

$\lambda_{\mathrm{i}}-\mathbf{i}^{\text {th }}$ eigenvalue

$\Lambda$ - total number of distinct eigenvalues; also used for cluster arrival rate

$\nu_{i}(t)$ - complex filtered additive white gaussian noise process at $i^{\text {th }}$ receiver antenna

$\phi_{h}(t)$ - multipath delay profile for a particular response $h(t)$

$\phi(t)$ - common multipath delay profile expression

$\phi(\mathrm{s})$ - natural logarithm of moment-generating function of decision variable $\mathrm{g}(\mathrm{s})$ 
multiplied by $\mathrm{s}^{-1}$

$\pi$ - constant $3.141592654 \ldots$

$\rho$ - instantaneous (short-term) signal to interference plus noise ratio at decision device input

$\rho_{\mathrm{d}}-$ instantaneous signal to interference plus noise ratio per sequence of $\mathrm{d}$ transmitted bits

$\rho_{\text {opt }}$ - optimized instantaneous (short-term) signal to interference plus noise ratio at decision device input

$\sigma^{2}$ - variance of gaussian process

$\sigma_{\mathrm{d}}-\mathrm{rms}$ delay spread

$\sigma_{\mathrm{d} t}-$ total delay spread

$\theta(t ; \tau)$ - continuous time multipath response phase function

$\tau$ - time delay variable

$\tau_{\mathrm{kl}}$ - arrival time of $\mathbf{k}^{\text {th }}$ ray within $1^{\text {th }}$ cluster

$\tau_{\mathrm{s}}$ - forward filter tap spacing

$\xi$ - standard deviation of large scale shadowing distribution; also used for path arrival rate in channel models; also used to denote AWGN component of decision variable

$\mathbf{x} !-x$ factorial

$[$.$] - long-term average$

[ . $]^{\prime}$ - first derivative

[. $]^{\prime \prime}$ - second derivative

1. 1 - absolute value or magnitude of a complex term

$\int_{a}^{b}-$ integral from $a$ to $b$

$\stackrel{b}{\prod}$ - product from $a$ to $b$

$\sum_{a}^{b}$ - summation from $a$ to $b$ 


\section{Chapter 1}

\section{INTRODUCTION}

In the past decade, the growth of wireless communications has been extraordinary. The great popularity of cordless telephones and cellular mobile radio telephones clearly demonstrates an intense and increasing demand. By 1987, 20 million [1, 2] cordless phones had already been sold in the U.S. while the number of U.S. cellular subscribers has been growing by $70 \%$ a year [3] over the past three years. This trend is expected to continue; 22 million U.S. cellular subscribers are forecast for $1995[3,4]$ while a European market of $\mathbf{5 0}$ million users of mobile communications services is predicted for the year 2000 [5]. This mass-market can only be satisfied if two conditions are met.

1. A shift is required from the present-day individual communication networks to networks giving integrated access to cellular, cordless, satellite and wireline (e.g. the emerging Integrated Services Digital Network) services. Combined with suitable intelligent network databases and personal numbering, this will usher in the era of universal personal and portable communications. As a service concept, personal communications $[6,7,8,9,10,11,12,13,14,15,16]$ represents the ability to initiate or receive calls, i.e. voice or other information such as data, facsimile, video etc., anywhere and to maintain one's identity throughout tie network. Information will therefore be routed to individuals via their own personal number regardless of the device used. This will contrast sharply with the current situation where information is routed to devices as is the case with today's wireline or mobile radio telephones.

2. To cope with the expected teletraffic demand, a shift must also be made from the present network infrastructures towards truly high capacity networks such as can be provided by microcellular system engineering techriques [17].

Such cellular systems [18], as have been devised and used in the last decade for vehicular mobile radio e.g. in the AMPS (Advanced Mobile Phone System) system 
$[19,20,21]$ in North America, TACS (Total Access Communication System) [20, 21] in the United Kingdom, NMT (Nordic Mobile Telephone) [20, 21] in Scandinavia and HCMTS (High Capacity Mobile Telephone System) [20 ] in Japan, are based on the concepts of frequency reuse and cell splitting. Frequency reuse refers to the use of radio channels on the same carrier frequency to cover different areas which are separated from one another by sufficient distances so that cochannel interference is not objectionable. Instead of covering an entire local area from one transmitter site with relatively high power, i.e. a centralized approach, the service provider can distribute transmitters of lower power throughout the coverage area. Each site then primarily covers some nearby subarea, or zone, or cell. In principle, the spacing of transmitter sites does not need in be regular and the cells need not have any particular shape. Through frequency reuse, a cellular mobile telephone system in a given coverage area can thus handle a number of simultaneous calls greatly exceeding the total number of channel frequencies. The factor by which the system capacity in simultaneous calls exceeds the number of allocated channels depends on several factors, particularly on the total number of cells. The latter can be increased by cell splitting, i.e. an area formerly regarded as a single cell is made to contain several smaller cells and can now use all the initial cell's channel complements. In this way, the system can be adjusted to a growing spatial traffic demand density (simultaneous calls per unit area) without any increase in the spectrum allocation. However, the price paid for the increased capacity obtained from cell splitting is a degradation of transmission quality resulting from a modification of the cochannel interference statistics and more frequent handoff.

Current cellular mobile radio systems were designed to work with vehicular radio equipment. These designs have inherent characteristics that limit their suitability for portable hand-held usage in an indoor environment such as an office building, factory, hospital or university. An efficiently designed, high-capacity indoor portable radio system is fundamentally different in many respects from a vehicular mobile radio system, even though it shares a similar frequency-reusing architecture. Some of the major distinguishing features of both systems are $[22,23]$ :

1. Users and traffic intensity: Vehicular systems cater to nigh-speed users moving in 
vehicles whereas portable systems provide service to slowly moving pedestrians. In addition, because vehicles are typically occupied only a fraction of the day, both average traffic intensity and total traffic per day are lower than for personal portables by up to a factor of ten.

2. Power: Since both a source of dc power (and its recharging) and a substantial stable mounting surface are available in a vehicle, its equipment typically can transmit at levels ranging from 1 to 10 watts. A portable unit with a lightweight, low-volume battery is not compatible with these power levels, and frequent recharging of the battery is not convenient. With the additional constraint $G$ ? health and safety with respect to the radiation hazard in a restricted multi-user environment, power levels of 1 to 10 milliwatts are more realistic for indoor wireless communications.

3. Coverage and cell size: The shorter range resulting from lower power and the increased traffic intensity implies a reduction in cell size for indoor communications. Very small microcells or picocells are being considered [24]; these might encompass a room, an office or a house and its immediate environment.

4. Fixed radio sites: These are typically expensive (cost of land and building) for vehicular mobile communications with base station antennas mounted on top of tall buildings. Radio sites are much cheaper for personal portable communications with base stations antennas mounted on ceilings or lamp poste.

5. Channel characteristics and impairments: The portable radio environment is qualitatively similar to the vehicular radio environment in that propagation .s dominated by large-scale shadowing effects and small-scale multipath fading effects. However, significant quantitative differences must be noted. As a portable system caters essentially to pedestrians, the channel variations are far slower than for a vehicular application. The portable system's ability to overcome very long and deep fades is therefore a key design constraint and adaptation issues are thus not as crucial as in a vehicular context. In addition, multipath dispersion within a building tends be less severe than in a vehicular application which affects the signaling rate at which equalization is required. Consideration must also be given to the excess attenuation factor for radio propagation into or within buildings and the larger mean cochannel 
interference levels resulting from the larger shadowing standard deviation [25].

The cordless phone is currently a popular means of wireless access in and around buildings. However, today's cordless phones do not use a cellular architecture and are simply cordless extensions of wireline mainstations. As such they also have significant limitations ${ }^{1}[1,2]$. Cordless phones do not save the investment in distribution facilities from the telephone network; the per-user wireline loop, including house/building wiring is still required. In addition, as they only have access to one radio channel, cordless phones are extremely vulnerable to interference, thus leading to a very limited user density.

Today's cordless telephones and cellular mobile radio systems are examples of firstgeneration technology [26], characterized by analog voice transmission and limited flexibility. In cellular systems such as AMPS, TACS and NMT, all communication between a mobile terminal and the network infrastructure is confined to a single channel. Network control messages, e.g. for handoff or power control, are carried on this channel, interrupting speech transmission and thus limiting system capacity. A number of second-generation systems [27] are currently on the drawing boards in several countries. All of them employ digital voice transmission. Second-generation vehicular mobile radio systems, such as the pan-european GSM (Groupe Spécial Mobile) system $[6,27,28,29,30,31,32,33]$ due to be launched in 1992 , the emerging dual-mode North American IS-54 standard ${ }^{2}[27,28]$ and a third upcoming Japanese standard ${ }^{3}$ $[27,28]$, will have dedicated channels for the exchange of network control information between mobile terminals and the network infrastructure. On the other hand, approaches to enhanced cordless telephony include CT2 $[6,27,28,21]$, a British standard and DECT, digital European cordless telephone [6, 27, 28, 34, 35, 36]. Second-generation cordless phone systems will now be cellular systems, i.e. they will have access to

Other current approaches to portable communications include radio paging, radio data systems, automatic call forwarding and citizens band (CB) radio. As with cordless phones, these schemes also have significant drawbacks [1, 2].

2 The Electronic Industry Association Interim Standard (IS-54) specifies dual-mode operation as it incorporates the first generation AMPS standard and adds a digital voice transmission capability to new subscriber equipment.

3 This standard will closely resemble $15-54$ with most variations due to the different spacing of carrier frequencies. 
many channels, automatically selecting, albeit without a dedicated control channel, the best available channel at the beginning of a call and switching to a new channel as conditions change during the call.

However, being optimized for voice transmission and with limited capacity for other kinds of information, second-generation systems will still satisfy only a limited number of portable communications needs. Moreover, it is expected [26] that, by the year 2000, demand for wireless services will overtake the capacity of second-generation technology. It is therefore the challenge of third-generation technology to provide universal, personal and wireless access to a variety of advanced information services. Such a network would serve urban areas as well as sparsely populated rural areas, operate efficiently indoors, outdoors or within vehicles and would serve stationary as well as high speed terminals.

In order to achieve this vision, a low-power tetherless access ${ }^{4}$ system must therefore be designed to complement current and future vehicular radio, mobile satellite, airto-ground systems as well as wireline networks in such a way that all of these can then be integrated into a unified access architecture. The design of a transceiver for such portable (indoor) radio links is the subject of this thesis. It should be noted that, as GSM provides roaming and authentication features necessary for personal communications [13], Britain has recently licensed a modified version of GSM, i.e. DCS 1800 [37], to provide low-power tetherless access. However, as such an approach involves modifying a vehicular design for portable use, it is not conducive to minimizing the mobiles and tase stations' cost, size, weight and power consumption which are all paramount criteria for portable communications. In this sense, it has been described as a "lost opportunity" [11]. A more reasonable approach [10] to providing ubiquitous personal communications is to continue to expand high-power vehicularoptimized mobile radio systems and to deploy separately (i.e. on dedicated frequencies) new tetherless access systems optimized for lightweight, pocket-size (e.g. 250 gr.)

Low-power tetherless access refers to portable radio links within as well as in the vicinity of buildings, i.e. in office buildings, in residences, shopping malls, airports, sidewalks, etc. As this represents communication primarily but not exclusively indoors, the term indoor wireless will be thus used interchangeably throughout this thesis with the temis low-power tetherless access and portable radio. 
personal communicators with maximum time of use before battery recharging or replacement. An integrated personal communications network (PCN) should therefore support, in addition to wireline communications, different tetherless communications systems optimized for their specific environments. This concept does not imply that all systems or subnetworks are owned or operated by the same business entity. However, the efficient interconnecting of subnetworks will require standardization of protocols and interfaces [38].

In such a network, the burden of call routing is placed on the overall network intelligence. As an example, the user's identity, i.e. his or her personal number, could be contained either in memory in the personal communicator or in a "smart card" [10] inserted into the set. When entering an automobile, the user could insert the communicator or the smart card into a receptacle in the vehicle's vehicular mobile unit. The latter would then initiate a data exchange with the vehicular mobile system indicating that the user is ready to receive calls at that unit. This information regarding the user's location would then also be passed on to the personal communication network intelligence in order that calls may be correctly routed. Hence, via automatic registration updating and personal numbering, personal communication networks ${ }^{5}$ will identify people instead of devices and will be able to switch circuits that are in use as a customer moves from one radio coverage area to another.

Within a given coverage area, one has to determine the process by which the common channel resource is shared. For indoor wireless communications, two different channel access schemes are currently being considered:

1. Time-division-multiple-access (TDMA): This technique is actually a combination of FDMA (frequency-division-multiple access) and TDMA; it is thus aiso referred to as FD-TDMA. The total frequency spectrum (typically 10 to $20 \mathrm{MHz}$ ) allocated to an arrangement of ports is divided into sets of time-division multiplexed channels. The TDM channels or sets are reused at ports separated in such a way to minimize cochannel interference. Such a frequency assignment scheme with TDM channels

5 In addition to routing calls via a personal number, network intelligence should also provide for call screening and altemate routing to voice storage, flexible integration of new services as well as for security and privacy. 
is already being used for vehicular mobile $[39,40]$. According to the bandwidth of the TDM channels, one may distinguish two sub-classes of this technique:

a. Narrowband TDMA: When the channel bandwidths are smaller than $1 \mathrm{MHz}$, propagation measurements ${ }^{6}$ indicate that the resulting fading process is essentially flat". Typically, narrowband TDMA schemes use a large number of channels ( 24 or 48 ) with data rates on each channel of 400 or 800 kbits per second (for a total bandwidth of 10 to $20 \mathrm{MHz}$ ). This technique has an advantage of simplified transceiver design (due to the flat fading); however, the relatively large number of channels complicates the channel allocation procedure and the transmission rates for each user are limited ardi typically fixed.

b. Wideband TDMA: When the channel bandwidths are larger than $1 \mathrm{MHz}$, the fading becomes frequency-selective which implies that equalization is now necessary ${ }^{8}$. However, the channel allocation scheme now involves fewer channels and users now have access to a flexible combination of transmission data rates to accommodate a variety of different applications (such as high quality voice, high resolution video, still pictures, facsimile, etc.).

2. Code-division-multiple-access (CDMA): This approach is based on direct sequence spread-spectrum modulation. Kavehrad et al. [43] have considered a centralized approach in which users communicate with a PBX through a common base station. Each user is assigned a unique spread-spectrum code sequence. These code sequences must have low (ideally zero) cross-correlation properties to ensure low interference between users' signals. More recently, it was proposed [44, 451 to embed such a scheme in a cellular hierarchy. Such a spread-sprectrum

6 As will be shown in Chapter 2, ms delay spreads rarely exceed 50 nanoseconds [41] in most indoor environments where small cells or larger cells with distributed antenna systems (leaky feeders) are used. The corresponding mean ms delay spread is typically 25 ns [41].

7 If the channel is considered flat when the ms delay spread, limited to 50 nanoseconds (with a mean value of 25 ns), is smaller than 5\% of the symbol interval $T$, and if the channel bandwidth is taken as $1 / T$, the channel can be said to exhibit flat or frequency-selective fading when the channel bandwidth is smaller or greater than $1 \mathrm{MHz}(2 \mathrm{MHz})$ respectively.

- The large irreducible error rates observed [42] when the ms delay spread, limited to $50 \mathrm{~ns}$ (with a mean value of $25 \mathrm{~ns}$ ) exceeds $5 \%$ of the symbol interval imply that equalization is necessary for signaling rates larger than $1 \mathrm{Mbits} / \mathrm{s}$ (or $2 \mathrm{Mbits} / \mathrm{s}$ ). 
cellular system would not however employ frequency-reuse; only separate bands for transmitting and receiving would be applied allowing the full (e.g. $25 \mathrm{MHz}$ ) band to be used in all cells with each having half this bandwidrh (e.g. 12.5 MHz) for transmitting and receiving. It has been claimed [45] that such a cellular CDMA system could achieve capacity improvements on the order of 4 to 6 over digital FD-TDMA and of nearly 20 over analog FD-TDMA.

At first glance, CDMA appears to hold an overwhelming capacity advantage. However, when it is noted that variations in power control with standand deviations of as little as 1 or $2 \mathrm{~dB}$ can result in capacity decreases of $30 \%$ or more [46], it is reasonable to assert that "the likelihood of realizing such high theoretical capacity estimates is small" [10]. Moreover, the use of multiple cell site and mobile set interaction, i.e. soft handoff, requires precise cell site synchronization [10]. Coupled with power control, these factors entail a complex, highly synchronized, multiple-feedback, dynamic system whose s:ability could be a concern. However, as spread-spectrum technology is very robust against interference, it may facilitate shared spectrum allocation [47]. Both channel access schemes (CDMA,FD-TDMA) are currently the focus of extensive research work and the jury is still out [48] as to which technique is the most desirable in order to provide personal communications service. This thesis contributes to this ongoing research effort by examining the TDMA option. In this case, wideband channels appear to be necessary in order to maximize system versatility and flexibility in an environment where data rates of up to $20 \mathrm{Mbits} / \mathrm{second}[23]$ are predicted for future applications. Hence, this thesis deals with a wideband TDMA approach to indoor wireless communication.

As will be detailed in Chapter 2, the main disturbances to be overcome in the wideband TDMA indoor channel are shadowing, frequency-selective fading and cochannel interference. With the aid of macroscopic diversity to alleviate the effects of shadowing, a receiver structure which conjugates post-detection space diversity and equalization into a scheme with multiple forward filters and a common feedback filter then represents an attractive technique to simultaneously combat signal fading, intersymbol interference and cochannel interference on the radio links between the portables and the base sta- 
tions. This equalizer configuration, first proposed by Monsen $[49,50]$ in the context of tropospheric scatter channels, provides an effective microscopic diversity order which is much larger than the explicit space diversity order. It cancels interference by exploiting the latter's short-term correlation (i.e. in terms of an average over a period smaller than the average fade duration) across both the explicit and implicit diversity paths which are uncorrelated in terms of their long-term means over the fading channel statistics. As minimum mean square error (MMSE) and maximum signal-to-interference-plus-noise ratio (SINR) adaptation criteria yield the same tap weights ${ }^{1}$ [50], this equalizer structure can be considered as the baseband counterpart of an optimum pre-detection combiner $[52,53]$ considered for narrowband channels. In the wideband case however, this optimum baseband combiner also combines the uncorrelated multipath returns thus helping to offset the explicit diversity sacrifice required for interference suppression.

Implementation of optimum baseband combining does not pose a problem at the base stations in terms of the spatial requirements for multiple antennas, but size constraints could make this impractical at the portables. Recently, compact built-in dual diversity antennas have been developed $[54,55]$ but the extension to the three-antenna case appears difficult considering the small volume (e.g. $300 \mathrm{cc}$ 's) of hand-held units. If a triple explicit diversity order is required to meet a given performance specification, current VLSI technology then makes channel coding a viable alternative to improve performance. However, the quasi-static nature of the portable channel precludes the use of interleaving to break up burst errors which can significantly reduce and even eliminate coding gains. This drawback can be alleviated if the diversity order is sufficiently large and if the code is sufficiently complex; e.g. for maximal-ratio combining of blockcoded FSK [56] on a flat-fading channel, an order-M non-interleaved coded system could then match the performance of an order- $(M+1)$ uncoded system. It is therefore of interest to determine if a similar conclusion can be held for the optimum baseband combiner used on a wideband TDMA indoor radio link. The main objective of this thesis thus consists of answering the following question:

1 This is true within a scaling factor [51]. 
On a wideband TDMA indoor radio link employing optimum baseband combining with explicit space diversity order 2, is channel coding a viable alternative to increasing the space diversity order to 3 even in the absence of interleaving?

Convolutional coding with Viterbi decoding is considered in view of its substantial performance / complexity advantage over block coding on both AWGN [57] and fading [58] channels. In addition, as complexity is a prime concern for low-power tetherless access, short constraint length codes are considered in order to minimize decoder complexity. With the effective diversity order now much larger than the explicit space diversity order of 2 , it is anticipated that even a simple code could yield positive coding gains. Finally, as conservation of portable set power is a key issue and in view of the quasi-static nature of the channel, coherent BPSK and QPSK are selected as modulation schemes.

\subsection{Thesis organization}

Chapter 2 provides a review of relevant background material and a thorough survey of the open technical literature. It consists of three major parts. First, the issue of characterizing the portable indoor channel is addressed. This involves reviewing the terminology used to describe most fading, time-dispersive channels and recalling the results of various propagation measurements in order to establish a realistic model. The influence of these particular characteristics on transceiver design is addressed. Comparison with the vehicular mobile radio channel's parameters and characteristics is emphasized. Cochannel interference statistics in a typical, high-capacity, frequencyreuse portable radio system are also examined. Second, the various countermeasures available to mitigate the channel's impairments, namely diversity, equalization and channel coding, are discussed. The principle of optimum baseband combining, which conjugates equalization and space diversity, is described. $A t^{t}$ id part highlights, in a brief manner, previous studies of the capacity of fading, tilı-dispersive channels. It is shown that the true potential of indoor radio links has not been determined. 
The following three Chapters each deal with a different technique to evaluate coded and uncoded optimum baseband combiner performance over wideband TDMA indoor radio links. In each Chapter, the method is first described after which results are presented.

- Following a description of the system model, Chapter 3 presents the derivation of both uncoded and coded optimum baseband combiner average bit error rate bounds. This analytical approach is based on a non-fading gaussian noise model of interference and on an assumption of ideal interleaving in the coded case. It quickly provides a large number of results without resorting to lengthy simulations.

- Chapter 4 then describes a corroborating bit-by-bit simulation where the effect of the non-interleaved channel is assessed with all analytical assumptions relaxed.

- Chapter 5 describes a semi-analytical approach involving the calculation, via the method of moments, of optimum baseband combiner performance over a static channel and Monte Carlo averaging over the fading distribution. In addition to providing results for both ideal interleaving and no interleaving, such a technique eliminates the non-fading gaussian noise characterization of interference inherent in the purely analytical approach of Chapter 3 while producing statistically significant results much faster than the simulation described in Chapter 4.

Finally, Chapter 6 contains the summary, conclusions and suggestions for further research.

\subsection{Thesis contributions}

This thesis presents a comprehensive investigation of the performance of compound strategies of channel coding, equalization and space diversity on frequency-selective fading indoor radio links subjected to cochannel interference and where ideal interleaving is not practically realizable. As will be discussed in Chapter 2, no such study has yet been undertaken, to the author's knowledge, for the indoor portable or yehicular mobile radio channels. This thesis thus provides the following contributions: 
1. The application of previous (uncoded) optimum baseband combiner derivations [50] for tropospheric scatter channels to frequency-selective, Rayleigh fading, indoor wireless radio links employing both BPSK and QPSK modulation (Sections 3.3 and 3.4).

2. The development of novel average bit error rate bounds for the optimum baseband combiner with convolutional coding and soft-decision Viterbi decoding on a frequency-selective, Rayleigh fading channel (Section 3.5).

3. A comprehensive set of analytical results is presented in terms of a variety of critical system parameters such as signal-to-interference ratio, interference-to-noise ratio, fixed receiver sampling phase, transmit/receive pulse shape, number of forward filter taps and signaling rate. The latter two parameters strongly influence the system's ability to exploit the channel's implicit diversity effect. BPSK and QPSK modulations are also compared based on a given rate of information throughput as well as in both interference-dominated and white noise-dominated situations. The option of using channel coding (with ideal interleaving) as an alternative to increasing the explicit space diversity order from two to three is also examined (Section 3.7).

4. A corroborating bit-by-bit simulation is performed to evaluate both uncoded and coded optimum baseband combiner performance. Coded performance is now evaluated with no interleaving. The performance of uncoded 2 and 3-antenna configurations is again compared to the 2-antenna coded situation. Results are also presented for different fixed receiver sampling phases and in both interferencedominated and white-noise dominated situations (Sections 4.2, 4.3 and 4.4).

5. Another bit-by-bit simulation is performed to evaluate the degree of cyclostationarity of the sum of two cochannel interfering sources received through independent realizations of the channel impulse response (Section 4.4.1). This effect can have a significant influence on performance sensitivity to receiver sampling phase.

6. A version of the method of moments, based on the numerical quadrature of a complex Laplace transform inversion integral and previously used [59] to evaluate the bit error rate of binary PAM on a simple binary symmetric channel, is extended 
to the case of BPSK or QPSK modulation with optimum baseband combining on an arbitrary channel. Monte Carlo averaging, over 10000 sets of portable-to-base station antenna realizations, is used to include the effects of fading (Section 5.2.1).

7. The method of moments, in conjunction with Monte Carlo averaging, is also employed to evaluate coded optimum baseband combiner performance under the assumption of either ideal interleaving or no interleaving. Novel analysis, although still based on the numerical quadrature of a complex Laplace transform inversion integral, is presented in order the evaluate the static channel error probability for both these coded cases. In this sense, expressions are derived for the momentgenerating function of the decision variate under the assumptions of both ideal interleaving and no interleaving. These results thus serve to establish upper and lower performance bounds for partial interleaving (Section 5.2.2).

8. The results of 6. and 7. are used to compare once more the performance of uncoded 2 and 3-antenna configurations to the coded 2-antenna situation. However, results are now expressed not only in terms of average bit error rate (as in 1. and 2.) but also in terms of outage rate which is the criterion of interest for portable communications. Average bit error rate and outage rate results are presented as a function of not only $\frac{E_{0}}{N_{0}}$ but also signal-to-interference ratio for one and two sources of cochannel interference (Section 5.4.1). The influence of continuously optimizing the receiver sampling phase, as opposed to a fixed sampling phase, is also assessed (Section 5.4.4).

9. Performance evaluations with the semi-analytical procedure (of 6., 7. and 8.) are carried out with a simple nine-tap channel model with fixed tap spacing and exponential delay profile as well as with a more complex cluster model based on measurements [60]. The comparison of results with both discrete models therefore provides an indication of how well the fast analytical procedure of 1 . and 2., which involves a simple continuous channel model with exponential delay profile, approximates a realistic channel (Section 5.4.2).

10. Comparison of the analytical results (of 1 . and 2.) with those of the semi-analytical procedure (of 6., 7. and 8.) yields an assessment of the influence of the selected 
interference model (e.g. non-fading gaussian noise) on performance predictions. This evaluation is crucial as such characterizations are common in order to make analysis tractable (Section 5.4.3).

11. As part of the suggestions for further research, a method to evaluate the informationtheoretic capacity bounds for indoor radio links is described (Section 6.2.4, appendices $D$ and $E$ ).

By evaluating the degree to which an optimum baseband combiner coupled with channel coding can mitigate the impairments and exploit the great potential provided by the implicit diversity of wideband TDMA indoor radio links, these results ${ }^{2}$ should therefore have a direct impact on the design of a wideband TDMA scheme for a highcapacity, portable, low power tetherless access communication system.

Contributions 1 to 5 are published in $[61,62]$ while 6 to 8 can be found in $[63,64]$. 


\section{Chapter 2}

\section{BACKGROUND and LITERATURE SURVEY}

\subsection{Introduction}

In this Chapter, a survey of relevant documents in the open literature is performed in order to ascertain the contribution of this thesis to the field. The performance of communication links is mainly limited by the properties of the transmission media. In order for system engineers to be able to optimize techniques to combat various impairments in the medium, it is imperative for the transmission channel to be satisfactorily characterized. Thus, although channel characterization and modeling are not the ultimate goals of this thesis, a survey of works on this subject is first done to determine and set bounds on the main disturbances to be overcome in the wideband indoor wireless channel. Subsequently, a thorough examination of countermeasures considered up to this point (for indoor and other related channels) is performed. Finally, the issue of channel capacity will be adiressed. It will be noted that the true potential of wideband indoor radio systems has not been completely determined.

\subsection{Characterixation of wideband indoor wireless channel}

Fundamental limitations on portable radio system parameters and on the application of radio link techniques result from the effects of radio propagation within and around houses and buildings. This is a very complex and difficult radio propagation environment because the shortest direct path between any pair of base station and portable set locations is usually blocked by walls, ceilings or other objects. Often, many attenuated propagation paths exist between any pair of lucations. The different propagation paths are produced by reflections from walls, ceilings and objects. Each path may have a 
different time delay and a different attenuation. The overall result is a complex and widely varying multipath and transmission channel between ports and portable sets. In the following pages, tha general model used to describe this channel will first be described. A review of various propagation measurements quantifying the parameters of this model as well as a brief discussion of the effects of cochannel interference will then follow.

\subsubsection{Channel model}

Prior to examining the specific characteristics of the indoor channel, it is worthwhile to briefly provide some background material relating to a commonly used model for a wide variety of well-known fading multipath channels [65], such as shortwave ionospheric HF (3 to $30 \mathrm{MHz}$ ), tropospheric scatter UHF (0.3 to $3 \mathrm{GHz}$ ) and SHF ( 3 to $30 \mathrm{GHz}$ ), and vehicular mobile radio $(800$ to $900 \mathrm{MHz}$ ). These dispersive radio channels have been described in terms of linear filters with randomly time-variant transmission characteristics $[66,65,67]$. A thorough treatment of time-varying channel characterization, detailing the various system functions, their inter-relationships and their physical representations is contained in [66]. Various symmetrical mathematical relationships between the channel system functions in the time and frequency domains were presented in Bello's work [66]. However, since the channel exhibits randomly time-variant characteristics, an exact description of its behavior is not possible without specifying the jrist multi-dimensional probability density functions for all random variables.

Certain s: ipli ications in the characterization can be effected, though, if the channel exhibits stacistical stationarity in the wide-sense. Fortunately, most radio channels possess stationarity over small spatial distances. This leads to a two-stage approach whereby the channel is characterized over a small distance (small-scale variations), where the char icl is considered stationary (at least in the wide sense), and then the large scale behavior (over difierent rooms, areas or buildings) is obtained by averaging over the small scale $\therefore .5 f$ if ors. This method was suggested by Bello

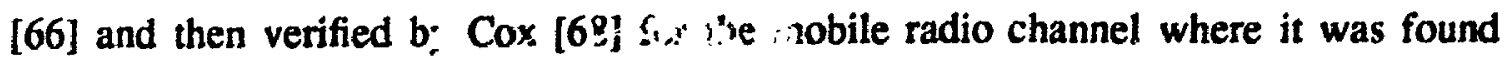


that power averages were approximately stationary over small-scale distances (i.e. for different colle:tions of data taken from the same area). In order to further simplify the characterization, the conditions that contributions from scatterers with different delays are uncorrelated anc that the fluctuations of the unresolved paths at each delay have Gaussian statistics, are also placed upon the channel. The Gaussian property results from the fact that the sum of a large number of signals with random amplitudes and uniformly distributed phases approximates a Gaussian process. By the central limit theorem [69], the approximation improves as the number of signals increases. The resulting channel model is thus called the Gaussian wide-sense stationary uncorrelated scattering (GWSSUS) model [66]. The low-pass equivalent time-variant response of the channel at time $t$ to an impulse applied at time $t-\tau$, is then a complex Gaussian process $h(t ; \tau)=\alpha(t ; \tau) e^{j \theta(t ; \tau)}=x(t ; \tau)+j y(t ; \tau)$ whose phase $\theta(t ; \tau)$ is uniformly distributed and whose envelope $\alpha(t ; \tau)$ is Rayleigh distributed when the independent gaussian processes $x$ and $y$ (each with variance $\sigma^{2}$ ) are assumed to have zero mean, which is realistic when scatterers are moving randomly and there is no direct path between transmitter and receiver. The Rayleigh probability density function (p.d.f.) of the envelope, conditioned by the large-scale variation, is $[70,71]$

$$
p\left(\alpha \mid \sigma^{2}\right)=\frac{\alpha}{\sigma^{2}} e^{-\frac{a^{2}}{2 \sigma^{2}}} \quad \text { for } \alpha \geq 0
$$

and has mean [71]

$$
\bar{\alpha} \mid \sigma^{2}=\sqrt{\frac{\pi \sigma^{2}}{2}}
$$

where the overbax denotes averaging over the channel (small-scale) ensemble statistics. This can be rewritten as

$$
p(\alpha \mid \bar{\alpha})=\frac{\pi \alpha}{2 \bar{\alpha}^{2}} e^{-\frac{\pi \alpha^{2}}{\bar{\sigma}^{2}}} \quad \text { for } \alpha \geq 0
$$

The distribution of $\bar{\alpha}$ represents the effect of shadowing, a large-scale variation of signal strength due to obstructions from large objects and terrain variations. Empirical observations $[72,1]$ have suggested modeling $\bar{\alpha}$ as a log-normally distributed random variable with a mean that varies inversely with a given power of distance between the 
transmit and receive antennas; i.e. the random variable $U=20 \log _{10}(\bar{\alpha})$ is normally distrikuted with probability density

$$
p(u)=\frac{1}{\xi \sqrt{2 \pi}} e^{-\frac{\left(u-m_{u}\right)^{2}}{2 \xi^{2}}}
$$

where the mean $m_{u}$ varies with distance $z$ as $m_{u} \propto z^{-n}$. Averaging the small-scale distribution of equation 2.3 over the large-scale distribution of equation 2.4 then yields the p.d.f. of the signal envelope with Rayleigh fading and log-normal shadowing as [73]

$$
p(\alpha)=\sqrt{\frac{\pi}{8 \xi^{2}}} \int_{-\infty}^{\infty} \frac{\alpha}{10^{0.1 u}} e^{\left.-\frac{\pi a^{2}}{4\left(11 u^{\prime 1} 1 u\right.}\right)} e^{-\frac{(u-m u)^{2}}{2 \xi^{2}}} d u
$$

When, in addition to randomly moving scatteners, there are fixed scatterers or signal reflectors in the medium, the small-scale distribution is more likely to be Rician which corresponds to the case where the two processes $x$ and $y$ now have non-zero means $m_{1}$ and $m_{2}$ respectively. The conditional small scale envelope p.d.f. is then $[74,71]$

$$
p\left(\alpha \mid \sigma^{2}\right)=\frac{\alpha}{\sigma^{2}} e^{-\frac{\left(\alpha^{2}+s^{2}\right)}{2 \sigma^{2}}} I_{0}\left(\frac{\alpha s}{\sigma^{2}}\right) \quad \text { for } \alpha \geq 0
$$

where $s^{2}=m_{1}^{2}+m_{2}^{2}$ and where $I_{0}(\cdot)$ is the zeroth-order modified Bessel function of the first kind. It should be noted if there is little or no motion in a building within which radio waves are transmitted between fixed locations, the fading distribution has been found [75] to differ substantially from the Rayleigh characteristic even without the presence of a dominant direct path. This is the result of the establishment of a physically stationary spatial pattern of field maxima and minima. In such a case, the absence of a dominant direct path implies that the fading distribution is not necessarily Rician but could be better described by other multipath fading characteristics such as the Nakagami (or $m$ ) distribution $[76,77]$ or even the Weibull distribution [76: As the perturbations due to movement of people and/or machinery increase, the distribution becomes Rayleigh (i.e. the mean ratio $\frac{s}{\sigma^{2}}$ of the specular to random multipath component decreases), even more so when the transmit and/or receive terminals are moving themselves. Throughout this work, analysis and simulations will be carried 
out under the assumption of Rayleigh fading. For a given median signal level, the probability of the signal being in a deep fade is stronger in Rayleigh than in Rician fading since the Rician probability distribution function has a much larger slope than the Rayleigh probability distribution function; for DPSK transmission on a slowlyvarying AWGN channel where the intersymbol interference caused by multipath and filtering is negligible, brief analysis [75] shows that this leads to lower average error rates in Rician fading. Hence, the assumption of Rayleigh fading generally represents the worst case (for average error rates in the absence of a strong specular component) and is therefore of greater concern in order to determine system performance.

The underlying assumptions in the GWSSUS model simplify the definition of a number of correlation and power spectral density functions used to characterize the multipath fading process. Since $h(t ; \tau)$ is wide-sense stationary and since the attenuation and phase shift associated with path delay $\tau_{\mathfrak{l}}$ is uncorrelated with the attenuation and phase shift associated with path delay $\tau_{2}$, the autocorrelation function of $h(t ; \tau)$ may be written as $[1,71]$

$$
\overline{h^{*}\left(\tau_{1} ; t\right) h\left(\tau_{2} ; t+\Delta t\right)}=\phi_{h}\left(\tau_{1}, \tau_{2} ; \Delta t\right)=\phi_{h}\left(\tau_{1} ; \Delta t\right) \delta\left(\tau_{1}-\tau_{2}\right)
$$

If we let $\Delta t=0$, the resulting autocorrelation function $\phi_{h}(\tau ; 0)=\phi_{h}(\tau)$ is simply the mean square value of the impulse response or the average power output of the channel as a function of the time delay $\tau$. For this reason, $\phi_{\mathrm{h}}(\tau)$ is called the multipath delay profile of the channel. In general, $\phi_{h}(\tau ; \Delta t)$ gives the average power output as a function of the time delay $\tau$ and the difference in observation time $\Delta t$.

The error rates for digital transmission through multipath channels, and particularly GWSSUS channels are most strongly dependent on the $\mathrm{ms}$ delay spread $\sigma_{d}$ or width of the power-delay profile defined as the square root of the normalized second central

3 As opposed to the ms delay spread, one may also define the total delay spread $\sigma_{d t}$ as the range of values of $r$ where $\phi_{h}(\tau)$ is essentially non-zero. 
moment of the impulse response functions, i.e. ${ }^{4}[66]$

$$
\sigma_{d}^{2}=\frac{\int_{0}^{\infty}(\tau-D)^{2} \phi_{h}(\tau) d \tau}{\int_{0}^{\infty} \phi_{h}(\tau) d \tau}
$$

where the average delay $D$ is the first central moment (mean) [66]

$$
D=\frac{\int_{0}^{\infty} \tau \phi_{h}(\tau) d \tau}{\int_{0}^{\infty} \phi_{h}(\tau) d \tau}
$$

At high signal-to-noise ratios where noise contribution to errors is negligible, delay spread results in irreducible error rates. This error rate depends somewhat on the shape of the delay profile (Gaussian, exponential, etc.) but it has been found, for a variety of modulation schemes and pulse shapes [42], to be far more dependent on the rms delay spread when the latter is smaller than a quarter of the symbol period. However, for larger values of $\sigma_{d}$ (relative to the symbol interval), the delay spectrum shape can have [79] a very significant influence on performance.

In an analogous fashion, the time-variant multipath channel can be characterized in the frequency domain. The autocorrelation function of $H(f ; t)$, the Fourier transform of $h(\tau ; t)$ with respect to $\tau$ in the frequency variable $f$, is referred to as the spacedtime spaced-frequency correlation function $[66,71]$ of the channel and is denoted by $\phi_{H}(\Delta f ; \Delta t)$. The assumptions of uncorrelated s;attering and wide sense stationarity respectively make $\phi_{H}(\Delta f ; \Delta t)$ dependent on frequency and observation time differences only. By setting $\Delta t$ to zero, the autocorrelation function in the frequency variable $\phi_{H}(\Delta f)$ is found to be $[66,71]$ the Fourier transform of the multipath delay profile $\phi_{h}(r)$. The width of $\phi_{H}(\Delta f)$ is thus proportional to the reciprocal of the total multipath delay spread $\sigma_{d t}$, i.e. the coherence bandwidth $B_{c}$ of the channel is given by [71]

$$
B_{c} \approx \frac{1}{\sigma_{d t}}
$$

4 Note that the moments are normalized to correspond to a unity area (i.e. unity total power) delay profile. The total average power received actually varies with the (large-scale) effects of shadowing, which is taken into account by the log-nomal distribution of the mean value of the received signal envelope as defined in equation 2.4 . 
The coherence bandwidth ${ }^{5}$ is thus a spectral measure of the correlation of the fading process. Just as two sinusoids with frequency separation greater than $B_{c}$ would be affected differently by the channel, a bandpass signal whose bandwidth is larger than the coherence bandwidth of the channel will undergo frequency-selective fading. Although the resulting combination of intersymbol interference and fading may make successful communication more difficult to achieve in a frequency-selective fading channel, the latter's significant delay spread (relative to the symbol interval) enables a properly designed receiver to exploit the dispersive channel's implicit diversity effect thus yielding improved performance. Hence, a signal bandwidth resulting in frequencyselective fading effectively makes it easier to exploit the true potential of the multipath channel, i.e. to approach its capacity.

The concepts of delay spread and coherence bandwidth deal with the variations in frequency of the channel characteristics (i.e. they were defined by setting the difference in observation time $\Delta t$ to zero). The time variations of the channel are evidenced as Doppler shifts. In order to relate the Doppler effects to the time variations of the channel, one may define the Doppler power spectrum $S_{H}(\chi)$ as the Fourier transform with respect to $\Delta t$ in the Doppler frequency variable $\chi$ of $\phi_{H}(\Delta f=0 ; \Delta t)$. If the channel is time-invariant, the spaced-time correlation function $\phi_{H}(\Delta t)$ has a constant value for any $\Delta t$ and $S_{H}(\chi)$ becomes a delta function $\delta(\chi)$. Therefore, when there are no time variations in the channel, there is no spectral broadening observed in the transmission of a pure frequency tone. The range of values of $\chi$ over which $S_{H}(\chi)$ is essentially non-zero is called the fading bandwidth or Doppler spread $[66,71] B_{d}$ of the channel. The latter's reciprocal is a measure of the width of $\phi_{H}(\Delta t)$ or of the coherence time $T_{c}$ of the channel, i.e. [71]

$$
T_{\varepsilon} \approx \frac{1}{B_{d}}
$$

5 The mathematical expression for coherence bandwidth depends on its exact definition. Defining the coherence vandwidth as the frequency separation leading to an envelope correlation of 0.5 [72] or yielding a given conditional outage probability [80], the measure $B_{e} \approx \frac{1}{2 \times \sigma_{f 1}}$ has been obtained. As such values can vary with the selected channel model (as in [80]), $B_{c} \approx \frac{f}{\sigma_{c t}}$ constitutes a "safe" upper bound. 
Clearly, a slowly changing channel such as the indoor channel, has a large coherence time or equivalently a small Doppler spread. The indoor radio channel varies slowly with time mainly because of people moving within the building, particularly in the vicinity of the transmit and receive antennas or in the open spaces, e.g. hallways between them. If people move very large metal objects or open or close metal doors in or near the path of the signal, the arrival times and strengths of various multipath rays can lead to the slow appearance and disappearance of deep fades. Most important however, is the motion of the portable unit itself. For stationary terminals with randomly moving scatterers, the Doppler spread has been found never to exceed $4 \mathrm{~Hz}$ [75]. This yields a coherence of at least $250 \mathrm{~ms}$; e.g. for a data rate of $1 \mathrm{Mbits} / \mathrm{s}$, the channel is time-invariant for at least 250000 bit intervals. Coherence time is reduced however when considering motion of the portable communications unit. A user walking at the regular pace of $5 \mathrm{~km} / \mathrm{h}$ through a medium-size office building has been found [41] to encounter a completely new channel every $72 \mathrm{~ms}$.

The dynamics of the indoor radio channel are therefore different in a fundamental way from those of the mobile radio channel where the mobile unit can be moving in and out of fades at tens of kilometers per hour. In the indoor channel, one can be stuck in a fade or, for that matter, get a strong signal, for a very long time, e.g. on the order of seconds or even minutes. However, one cannot rely on the channel always remaining static for such long periods, i.e. coherence times should not be assumed to exceed 100 $\mathrm{ms}$ and $\mathbf{2 5 0} \mathrm{ms}$ for moving and stationary users respectively. Although the channel dynamics are different, the portable radio environment is still qualitatively similar to the mobile (vehicular) radio environment in that propagation is dominated by the effects of shadowing and reflections from walls, objects and the ground. Therefore the GWSSUS model may be also be applied over small areas for indoor channels [81]. However, in the portable environment, antenna heights are lower, or antennas are located within buildings, and distances between transmitters and receivers are shorter than in the mobilk environment. These physical differences cause quantitative differences between the large scale characteristics of the portable (indoor) and mobile environments. In the indoor case, moving to a different room (or a different street or building) results 
in different dominant reflecting surfaces at longer delays and in different attenuations of shorter, more direct paths. Therefore, averaged power delay profiles and delay spreads in different rooms may be quite different. These observations point out that, just as the vehicular mobile radio environment, the multipath propagation process in the indoor medium is only (approximately) stationary over small areas and is highly non-stationary over large distances, from room to room, from building to building, etc. Hence, two-step modeling of the indoor channel is reasonable; small areas can be modeled as Rayleigh fading GWSSUS channels with log-normal shadowing and fixed delay profiles and delay spreads. Then distributions of delay spread which, as mentioned earlier, has a greater influence on error rate than delay profile (at least for $\sigma_{d} \leq 0.50 T$ ) can be used to determine performance cver a specified portion of the overall portable radio environment.

\subsubsection{Propagation measurements and their influence on channel modeling and system design}

Over the past six or seven years, various propagation measurements have been carried out to characterize the attenuation, fluciuation and impulse response characteristics of the indoor radio channel at various operating frequencies. Results regarding the attenuation of microwave CW signals propagating within $[82,83]$ or into [84] various buildings were reported first. The work of Alexander [83], which was performed at 900 $\mathrm{MHz}$, is particularly useful since it gives the power law $z^{-n}$ and standard deviation $\xi$ of log-normal shadowing (equation 2.4) representing the signal attenuation as a function of distance in a wide variety of rooms and offices with different construction materials within the partitions. The signal power was found to vary as much as the sixth power $(n=6)$ of distance in buildings with steel partitions and as little as the first $(n=1.2)$ power of distance in an open hangar. In the latter environment, the standard deviation was only $1 \mathrm{~dB}$ whereas it increased to $16 \mathrm{~dB}$ in the steel building. The average (over about 20 different building types) power law exponent and average standard deviation were in the range of $n=4.5$ and $\xi=10 \mathrm{~dB}$. The magnitude of the distance dependence exponent appears a little greater and the standard deviation somewhat larger in the 
(indoor) portable environment than in the (outdoor) vehicular environment [85]. The stronger distance dependence is probably a result of the lower port antenna heights [86] with respect to the ground and the deeper immersion of the port antennas within the buildings. The larger standard deviation undoubtedly results from the variability of the additional attenuation through walls into the interiors of houses and buildings.

The first multipath delay spread measurements were reported by Devasirvatham $[81,87]$ who made extensive impulse response measurements at $850 \mathrm{MHz}$. It was found that the rms delay spread could be as high as $420 \mathrm{~ns}$ (in a large building) when there was no direct line of sight between the transmit and receive antennas; however, when there was a good direct path, the mean Ims delay spread was found to vary from $25 \mathrm{~ns}$ in a medium size office building to about $125 \mathrm{~ns}$ in a large building. These measurements were made in buildings with wood studded plasterboard walls; if metallic partitions are present, Alexander and Pugliese [88] indicated that the multipath could be more severe. Building-dependent variations were also observed recently by Gutzeit [89] when investigating outdoor to indoor propagation at $900 \mathrm{MHz}$.

In addition, Saleh [60] found ms delay spreads of up to 50 ns (total delay spread of up to $200 \mathrm{~ns}$ ) at $1.5 \mathrm{GHz}$ in a two-story medium size building. As with Alexander's results, the signal attenuation seemed to obey an inverse distance power law with an exponent of 4 or 5 . Saleh's results were used to elaborate a model of the indoor channel where the received signal rays arrive in clusters. The rays have independent uniform phases and independent Rayleigh amplitudes that decay exponentially with cluster and ray delays. The clusters (formed by the building superstructure) and the rays (formed by objects close to the transmitter and receiver) within a cluster are modeled as Poisson processes with different but fixed rates. However, more recent measurements by Ganesh et al. $[90,91]$ on manufacturing floors indicate that the path ampliturles may follow a log-normal rather than a Rayleigh distribution and that the ray arrival process may be more accurately described by a modified Poisson process such as the one proposed by Suzuki [92] for urban radio channel modeling. In fact, modeling a channel in such a way, i.e. as a collection of discrete resolvable paths, has also been perfomed for the (vehicular) mobile radio channel [93] where discrete correlated rays have log-normal 
amplitudes. A discrete model such as Saleh and Valenzuela's cluster model [60] can be made to approach the continuous GWSSUS [66] model by letting the ray arrival rate approach infinity [94]. In general, this type of discrete modeling is convenient for simulation whereas continuous channel modeling is more suitable for mathematical analysis [94]. It should also be noted that, as opposed to time domain models, frequency domain models which closely match empirical data have also been proposed [95, 96].

The underlying vision in most initial measurements was that of an indoor radio communications system serving an entire building, or large Sections of building from a centrally located antenna. This is a desirable objective from the twin points of view of reducing the required number of base stations and minimizing the amount of wiring. However, one may identify three drawbacks in this approach:

1. The building-dependent variations of $\mathrm{ms}$ delay spread (at least a factor of 15 when comparing $25 \mathrm{~ns}$ to $420 \mathrm{~ns}$ ) complicates transceiver design.

2. The large propagation losses (e.g. in large buildings) would necessitate the radiation of an unreasonable amount of power to obtain satisfactory SNR. As noted in Chapter 1, this may create adverse health effects in a multi-user environment while the large power consumption may demand the use of an unreasonably bulky battery.

3. The signal from a central antenna serving all of a given building would surely penetrate adjacent buildings where different frequency bands would have to be employed. This would require a citywide coordination of the frequency assignment, a difficult task in view of variable radio communication needs in each building.

To overcome these problems, it was proposed 'y Saleh et al. [41] to either 1) divide the building into very small cells ${ }^{6}$ (such as picocells) with adjacent cells using different frequency bands or 2) continue using large cells but replace the single antenna of each central station by a distributed antenna system or leaky feeder that winds its way through the hallways of the building. It was found that, with these techniques, the rms delay spread becomes limited to $50 \mathrm{~ns}$, with a typical mean value of $25 \mathrm{~ns}$, even in large buildings. The choice between these two techniques is essentially a question of

6 Cell size can be effectively controlled not only by changing the transmitted power but also by modifying the base station antenna height [97]. 
user density. The picocell approach has the advantage of reusing the same frequency band in several widely separated cells thereby increasing spectral efficiency and system capacity [17]. On the other hand, the leaky feeder approach may be selected if the area covered by each cell is geographically too small to have a sufficient number of users to justify 1 ) the cost of its central base station and 2) the more complex channel allocation procedure resulting from more frequent handoff [98] in small cell systems. A wideband TDMA channel access scheme tends to counterbalance (in terms of the Jemands on channel allocation) the effect of cell size reduction and as the numbers of users (and their needs) grow, the picocell approach appears feasible and necessary in order to provide flexible and versatile indoor personal communications. Future cellular systems will therefore contain large cells, microcells and picocells, the latter two being used where user-density is highest.

The reduction in delay spread variability resulting from the picocell and/or leaky feeder approaches implies that reasonable estimates of system performance over a number of different indoor environments can be obtained with a fixed value of $\sigma_{d}$, i.e. without averaging over a distribution of delay spread as suggested at the end of Section 2.2.1. As its influence on performance can be significant when the delay spread is large, one must also determine the shape of the average delay profile to be used in simulations or analytical studies. Attempts to synthesize power-delay profiles from the geometry of a location have not been very profitable [87]. The danger that purely analytical studies could grossly underestimate the size of the impulse response profiles at any location is very real, due to the frequent unexpected reflections encountered. Consequently, a number of simple profile functions have been proposed. Normalized to unity area, these are

$$
\begin{aligned}
& \frac{1}{\sqrt{2 \pi \sigma_{d}}} e^{-\frac{1}{2}\left(\frac{r}{\sigma_{d}}\right)^{2}} \quad(\text { Gaussian })
\end{aligned}
$$

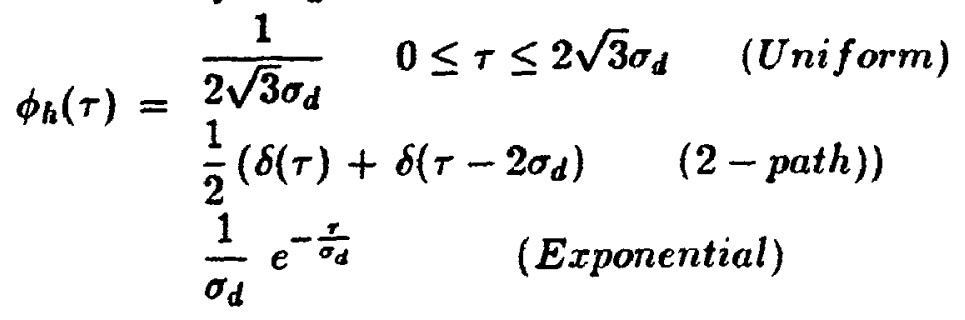


Bello and Nellin [99] first used the Gaussian shape, Bailey and Lindenlaub [100] then introduced the uniform shape and based on measurements [101] with short mobileto-base distances, the 2-path and exponential were later proposed. Such delay profiles shapes have been used in studies of both vehicular mobile radio [79] and portable indoor wireless [102]. Various measurements and an examination of more than 1400 profiles [87] have shown that profiles that occur in reality are most often a mixture of these cases.

The various parameters characterizing the indoor wideband channel are aiso dependent on the operating frequency of the proposed system. At this time, no portion of the radio spectrum has been definitely allocated for universal portable communications as described in this work. The major factors affecting a choice of operating frequencies from the technical standpoint are [1]

1. Antenna efficiency: The efficiency of handset size antennas, i.e. the ratio of radiated power to the sum of radiated power and the power of antenna losses, will be less than $50 \%$ [103] for frequencies below 300 or $400 \mathrm{MHz}$.

2. Signal decorrelation: If space antenna diversity is used [55, 104], an antenna separation of a quarter wavelength for signal decorrelation within a 15 to $20 \mathrm{~cm}$ set requires frequencies above $500 \mathrm{MHz}$.

3. Signal attenuation: For a given effective radiated power, mean signal levels for indoor radio environments may drop by as much as $5 \mathrm{~dB}$ from $500 \mathrm{MHz}$ to 100 $\mathrm{MHz}$ and attenuation levels also increase with frequency above $1 \mathrm{GHz}[1]$. Above $10 \mathrm{GHz}$, indoor coverage outside of one room thus appears unlikely.

4. Fading dynamic range: Bultitude et al. [105] have found that the dynamic range of $\mathrm{CW}$ (continuous wave) envelope fading is about $10 \mathrm{~dB}$ greater at $1.7 \mathrm{GHz}$ than at $900 \mathrm{MHz}$. This results from the power of the scattered component of received signals being greater in the higher frequency band and is evidenced by more Rayleigh-like p.d.f. (with lower $s / \sigma^{2}$ in equation 2.6) as opposed to Rician. This indicates that the quality of indoor channels could be marginally better at 900 
$\mathrm{MHz}$ than at $1.7 \mathrm{GHz}$.

5. Delay spread variability: Again, Bultitude et al. $\{105]$ have found that the variability of $\mathrm{rms}$ delay spread is greater at $1.7 \mathrm{GHz}$ than at $900 \mathrm{MHz}$ in two different buildings. Theretore, coverage for an indoor wideband system could be less uniform at $1.7 \mathrm{GHz}$ than at $900 \mathrm{MHz}$. However, the mean value of rms delay spread has not been found to vary significantly [106] over this frequency range.

6. Man-made noise: Man-made noise decreases rapidly with increasing frequency [72], with a rate of change at least as rapid as $1 / \mathrm{f}^{2}$. Man-made noise is emitted from equipment such as arc welders, automobile ignitions and incandescent light dimmers. It is often produced by sparking or rapid switching of electric currents.

All these factors suggest a rather broad optimum range of operating frequencies [1] from about $500 \mathrm{MHz}$ to 1 or $2 \mathrm{SHz}$ with acceptable operation possible up to 4 or 5 GHz. This range should yield several possible candidates for reallocation to a portable communications service. For example, in the early 1990's, there are still many unused UHF television channels. With the rapid expansion of cable TV and the coming of satellite direct broadcast TV, the UHF TV channels are likely to be even less used and therefore could be made available for a low-power tetherless access system. However, the perspective of bit rate intensive future applications such as high resolution video is currently fostering interest in frequency bands of $20 \mathrm{GHz}$ to $60 \mathrm{GHz}$ where large bandwidths are readily available. These channels have not yet been characterized thoroughly and the channel models used in this thesis will therefore apply to carrier frequencies in the $900 \mathrm{MHz}$ to $2 \mathrm{GHz}$ range.

\subsubsection{Cochanr.el interference considerations}

When a cellular system is first established, i.e. when the number of users is very small, there is normally little frequency-reuse. Since each initial cell is relatively large, the total number of cells needed to span the desired coverage area does not greatly exceed the number of channel into which the total spectral allocation is partitioned. Hence, until a certain amount of growth in traffic occurs, the main channel impaiment to contend with is ambient noise, both inevitable receiver thermal noise and man-made 
environmental noise. As the traffic demand increases, smaller cells are used to increase system capacity and the amount of frequency reuse increases. Cochannel interference thus becomes a fundamental consideration for high-capacity small-cell systems in terms of borh user access [107] to the network and achievable performance on the radio links.

The radio links typically will fail if an adequate average signal-to-interference ratio $\frac{S}{T}$ is not maintained. Since the source of the cochannel interference impairing a radio link is (on average) at least as distant from the receiver as the channel reuse ratio [18] i.e. the ratio of the distance between the centers of neighboring cochannel cells to the cell radius, the average $\frac{S}{I}$ on a given link is highly dependent on the large scale signal variations. In fact, Muammar and Gupta [25] have established that users will encounter higher levels of cochannel interierence as the shadowing standard deviation $\xi$ increases. Since the latter and the average traffic intensity, i.e. the average number of active cochannel cells, are generally larger for indoor portable communications as opposed to vehicular mobile, it is therefore even more important for indoor wireless communications to find a radio link communication technique which can tolerate high cochannel interference levels.

The $\frac{S}{I}$ ratio can be improved slightly by changing the cell shape [108] or by using overlaid cells [109]. In addition, since portable sets often can be served from more than one port, particularly when located at nearly maximum distance from the nearest port, macroscopic diversity can be used to mitigate these large scale variations by selecting the port-to-portable path resulting in the best signal according to some quality measure [110]. In most studies [111, 112], it is generally assumed that the local mean signals ${ }^{7}$ (desired or interfering) 1 om different ports are independent due to the separation of the propagation paths. The effectiveness of this macroscopic diversity can however be reduced by partial correlation $[113,114]$ among the large scale variations for the different ports.

Many studies of macroscopic diversity architectures consider the sum of cochannel interference signal envelopes $y=\sum_{i=1}^{N} \alpha_{i}$ from multiple interfering ports as having gaussian characterisucs. However, the distribution of the sum of signal envelopes $\alpha_{i}$

7 l.e. the log-nomally distributed mean value $\bar{a}$ of the received signal envelope. 
from $N=6$ cochannel interfering cells, where the p.d.f. of each $\alpha_{\mathrm{s}}$ is given by equation 2.5 (i.e. Rayleigh fading and log-normal shadowing), has been found to be $[25,115$, 116] closely approximated by a gaussian distribution for $\xi=0 \mathrm{~dB}$ but more by a $\log$-normal distribution for $\xi>0 \mathrm{~dB}$. Hence, the sum of six signal envelopes is not necessarily a normal variate as might be concluded from the central limit theorem [69]. Furthermore, Bernhardt [117] has shown that the cochannel interference sum is most often dominated by a single interferer. Berni.ardt's data, reproduced in Figure 2.1. was obtained from a Monte Carlo simulation of a frequency reuse radio system with four-branch macroscopic diversity, twenty surrounding cochannel interfering diversity groups, a channel set size of 25 , a path loss exponent of $n=4$ and a log-normal standard deviation of $\xi=10 \mathrm{~dB}$. It can be seen that for $15 d B \leq S \leq 20 d B$, one interferer makes up at least $50 \%$ of the total interference power with probability 0.85 . The latter value increases to 0.90 and to 0.95 for $10 d B \leq \frac{S}{T} \leq 15 \mathrm{~dB}$ and $5 \mathrm{~dB} \leq \frac{S}{T} \leq 10 \mathrm{~dB}$ respectively. These considerations suggest that the gaussian assumption of cochannel interference signal envelope may therefore not be very realistic in the context of a high capacity frequency reuse portable radio system.

Adjacent channel interference can also be a significant impairment in cellular radio systems. It is usually controlled by selecting a modulation scheme with low far-out spectral energy as well as by appropriate pulse shaping and filtering. When adjacent channels are not used at the same fixed radio sites as should be the case for a low power tetherless access system [1], adjacent channel interference may also be reduced by maximizing the distance separation between adjacent channel sites. Under this assumption of adjacent channels not being used at the same radio sites, cochannel interference should be a far greater impairment than adjacent channel interference which is therefore not addressed in this thesis. It should be noted however that the techuiques discussed in Section 2.3.2 to mitigate the effects of cochannel interference can also be used to combat adjacent channel interference [118]. 


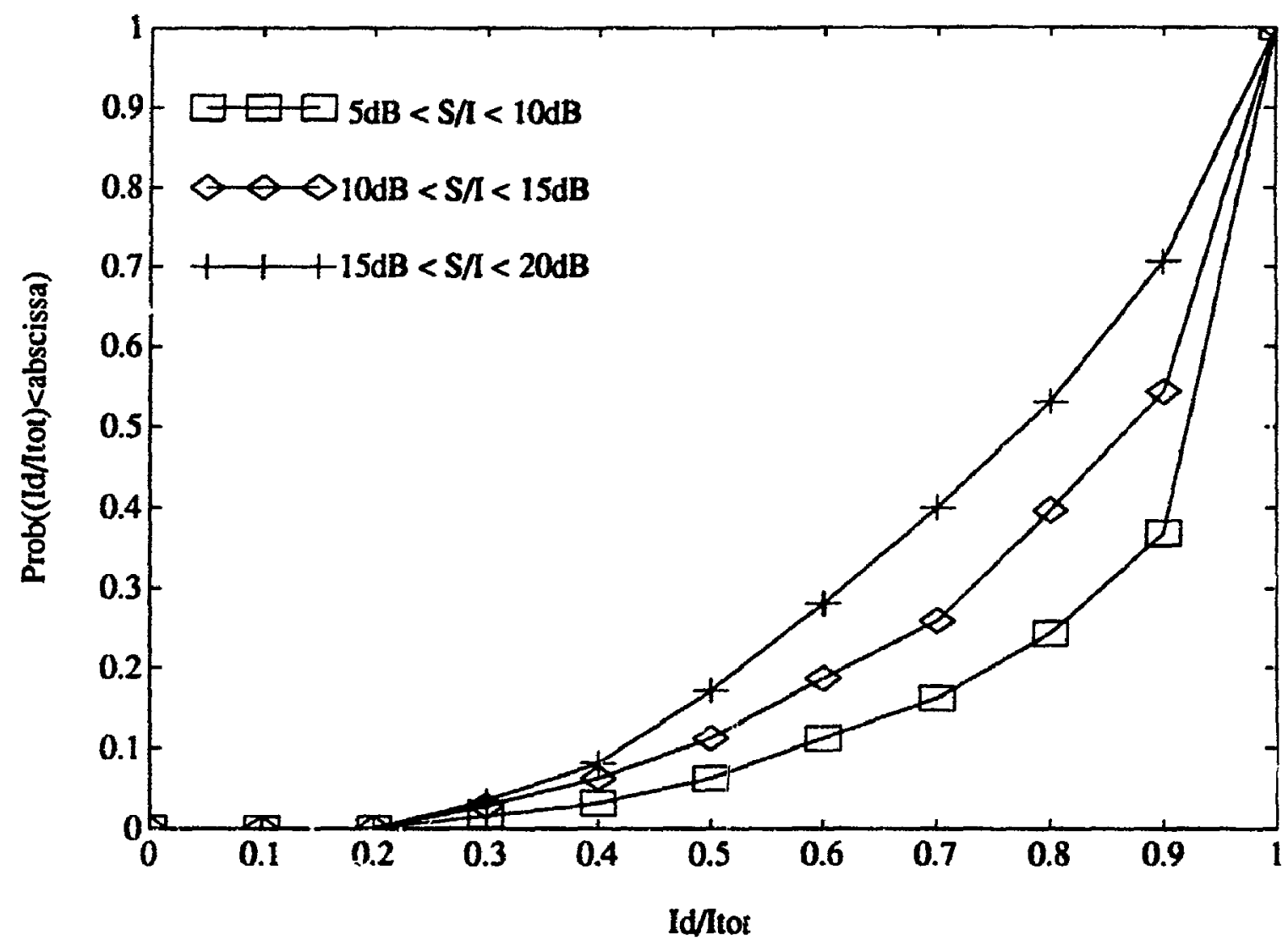

Figure 2.1 Distribution of ratio of dominant interferer power (Id) to total interference power (Itot). Data obtained from Bernhardt [117] from a simulatiun of a frequency reuse system with 4-branch macroscopic diversity, 20 active cochannel cells, path loss exponent $n=4$ and log-normal standard deviation $\xi=10 \mathrm{~dB}$. 


\subsubsection{Summary of indoor wideband channel characteristics}

From the preceding discussion, one may therefore identify three important impairments in the wideband indoor channel, i.e.

1. Shadowing: This is a large-scale variation in signal strength caused by obstructions from large objects or terrain variations. It is modeled using a log-normal distribution with a mean which a function of distance $z$ according to a power law $z^{-11}$ and standard deviation $\xi$. Measurements have shown that the values of $\mathrm{n}$ and $\boldsymbol{\xi}$ can be as large as $n=6$ and $\xi=16 \mathrm{~dB}$ in indoor environments, thus creating more severe shadowing than in the vehicular mobile radio environment.

2. Frequency-selective fading: Fading, a small-scale variation due to the random phase additions of multipath signals, can be Rayleigh or Rician depending on the availability of a direct path and the motion of the portable unit (and to a lesser degree, to the motion of random scatterers). The time dispersion characteristics of the indoor channel are described by rms delay spreads rarely exceeding 50 ns in a small-cell system and with a typical mean value of $25 \mathrm{~ns}$. As high data rates (and therefore large bandwidths) are being considered, the fading is frequencyselective resulting in intersymbol interference. The time variations of the fading characteristics are also far slower than in the vehicular environment, with coherence times in the order of $70 \mathrm{~ms}$ for a walking user and $250 \mathrm{~ms}$ for stationary terminals.

3. Cochannel interference: It constitutes a major impairment in high capacity smallcell systems and is highly dependent on the shadowing characteristics. Since the latter is more severe in the indoor medium, so will the effects of cochannel interference. It is therefore important for indoor wireless system designers to develop radio link techniques which can provide satisfactory performance at low $\frac{S}{T}$ ratios. 


\subsection{Countermeasure techniques for wideband indoor channel}

A high-capacity UDPC system must therefore counteract the effects of shadowing, frequency-selective fading and cochannel interference. A combination of macroscopic and microscopic diversity has been shown [119] to be effective against shadowing and signal fading and should aiso be desirable for the wideband indoor channel. As discussed earlier, macroscopic diversity alleviates the effects of shadowing by, in most applications, selection of the best portable-to-port path among a number ports or base stations. Using a parametric cost model [120] based on the variable $V$ and fixed $F \operatorname{costs}^{8}$ of base stations, it was found $[111,120]$, for a given set of propagation parameters and coverage criteria, that macroscopic diversity is an effective cost saving strategy (in terms of total cost per unit area) when the ratio $\frac{V}{F}$ is relatively small and the main channel limitations are desired signal strength and cochannel interference. However, in a wideband TDMA implementation, multipath delay spread also limits system performance. With the use of adaptive equalization, the $\frac{V}{F}$ ratio is increased, indicating that it may be more cost-effective to concentrate on microscopic diversity instead of increasing the macroscopic diversity order in a wideband TDMA scheme. Hence, the remainder of this work will deal with techniques to combat frequencyselective fading and cochannel interference on a given portable-to-port radio link, assuming fixed average signal and cochannel interference levels which are dependent on shadowing effects and the (fixed) order of macroscopic diversity.

\subsubsection{Techniques to combat dispersive fading}

\subsubsection{Diversity}

Let us now consider the use of microscopic diversity at the radio link level to reduce the effects of signal fading. The context of flat fading will be examined first, after which the limitations imposed by delay spread will be discussed. A variety of diversity

- Fixed costs include the housing, power supply, various RF transmit and receive amplifiers, connection to commercial ac power, etc. Variable costs refer to the transmitter modulator and RF stage, the receiver IF and demodulator, plus per-branch circuitry at the macroscopic diversity combiner. 
approaches have been considered for mobile radio [72] and for other fading radio channel environments [65]. Many of these approaches also have potential applicability for use in the portable radio environment [121]. Diversity techniques can be classified in many ways in addition to the macroscopic/microscopic classification.

One diversity classification is by the method used to provide the independently varying signal paths. These include the use of different frequencies, different times of transmission, or different antennas with a sufficiently large spatial separation or with different polarizatious or pattern orientations ${ }^{9}$. Frequency diversity (slow frequency hopping) in conjunction with burst error-correcting codes $[122,80]$ has recently been proposed for a flat fading ${ }^{10}$ TDMA indoor radio application. However, such a scheme can require excessive bandwidth to achieve decorrelation with the small delay spreads ${ }^{\text {" }}$ typically encountered in the indoor channel. As the number of users hopping through a preset cyclical sequence of frequencies and their needs (in terms of data rate) grow, the required total frequency allocation could be large. Frequency diversity can thus be inefficient in its use of the scarce frequency spectrum and time diversity would require excessive time separation to achieve deconelation in the quasi-static indoor channel; hence antenna diversity, and particularly space diversity in view of the availability of compact dual diversity antennas with gcod decorrelation characteristics [54, 55], is attractive for portable radio links. As the fading statistics of the signals receiven at two antennas are nearly independent when the spatial separation is at least a quanter wavelength [104], the required separation is about $8.3 \mathrm{~cm}$ at $900 \mathrm{MHz}$ and is smaller at higher frequencies.

Another classification involves the method used to combine the diversity signals; the most common combining techniques [72] are selection, switched, equal-gain or maximal ratio. These can be further classified into predetection and postdetection (baseband) combining techniques. Predetection techniques are most often preferred as,

9 Polarization and field diversity may be considered special cases of antenna diversity as more than one antenna is used.

10 This limits the signaling rate to approximately $1 \mathrm{Mbits} / \mathrm{s}$.

11 With a typical mean ms delay spread of $25 \mathrm{~ns}$, using $B_{c} \approx \frac{1}{2 \pi 0_{\Delta t}}$ implies frequency hops of at least $6.4 \mathrm{MHz}$. 
although cophasing is required, they do not involve multiple carrier recovery loops (for coherent detection) and repetitions of other receiver elements. With selection diversity, the receiver simply selects for reception the signal from the antenna with the highest signal power. Under the assumption of independent fading at each antenna, the outage probability for selection diversity with $M$ antennas is the outage probability of a single antenna raised to the $M^{\text {th }}$ power [72]. Since the probability, in Rayleigh fading, of receiving a signal $\chi \mathrm{dB}$ below the average signal power is approximately $10^{\frac{-x}{30}}$ :72], the requirer! average received signal power for a $10^{-4}$ outage probability at a given BER need only be 20 (or 10) $\mathrm{dB}$ higher for selection diversity with 2 (or 4) antennas than required for that BER without fading. This compares with $\mathbf{4 0} \mathbf{d B}$ with no diversity. Implementation of selection diversity implies the signal strength at each antenna must be continuously monitored, thus requiring $M$ receivers ${ }^{12}$. However, the outage probability of selection diversity can also be achieved with switched diversity, whereby a single receiver switches from one antenna to another when a given antenna is in a fade. Somewhat better performance can be obtained by using $M$ receivers with equal-gain combining where the diversity branch outputs are weighted equally before being summed or maximal-ratio combining where the branch gains are continuously and adaptively adjusted to produce the highest signal-to-noise ratio ${ }^{13}$. The analysis of Winters and Yeh [102] has shown that with maximal-ratio combining, the required average signal power for a $10^{-4}$ outage probability at a given BER need be 18 (or 6) $\mathrm{dB}$ higher with 2 (or 4 ) antennas than required for that BER without fading. The advantage of maximal-ratio combining over selection or switched diversity is seen to be fairly small with a diversity order of two, although the margin increases with an increase in diversity order. These considerations, along with the results of Cox [123], imply that the diversity order is far more significant than the type of combining strategy in terms of mitigating the effects of signal fading.

When the channel is frequency-selective, i.e. when the delay spread becomes significant with respect to the symbol interval, intersymbol interference places a lower

12 For predetection combining, this implies $M$ front-end mixers and IF amplifiers.

13 This involves weighting each branch in proportion to its signal-to-noise ratio. 
limit on the achievable average bit error rate. Unacceptable irreducible error rates, i.e. larger than $10^{-4}$ and as high as $10^{-2}$, have been observed [42] for different modulation schemes (BPSK, QPSK, GMSK) and SRC pulse excess bandwidths when the rms delay spread exceeds $5 \%$ of the symbol interval ${ }^{14}$. Studies $\{102\rceil$ of (predetection) maximal-ratio combining with coherently detected BPSK and QPSK in a slowly-varying frequency-selective environment without any cochannel interference, have shown that diversity can be used to raise the maximum permissible data rate. In addition, as QPSK transmits two bits per symbol, it provides a higher maximum data rate than BPSK. However, cross-rail interference is present in QPSK which causes the maximum data rate (for a $10^{-4}$ outage probability at a $10^{-4}$ BER) with QPSK to be somewhat less than twice that of BPSK, i.e. about $1.4^{15}$. But, based on the same bandwidth efficiency in terms of bits/sec/Hz i.e. by comparing 0\% SRC-BPSK with $100 \%$ SRCQPSK, QPSK then has approximately three times the maximum data rate of BPSK. The results of Winters and Yeh [102] also indicate that, using QPSK with 100\% roll-off SRC pulses and two-branch $(M=2)$ maximal ratio combining, the maximum data rate (for a $10^{-4}$ outage probability at a $10^{-4}$ BER) in a typical building with a mean $\mathrm{rms}$ delay spread of $25 \mathrm{~ns}$ is $8 \mathrm{Mbits} / \mathrm{s}$ corresponding to a $4 \mathrm{Mbits} / \mathrm{s}$ symbol rate. With $M=4$, this value increases to $16 \mathrm{Mbits} / \mathrm{s}$ and it reaches $22 \mathrm{Mbits} / \mathrm{s}$ with $M=6$. With $0 \%$ roll-off SRC pulses, the corresponding maximum data rates are $5.5 \mathrm{Mbits} / \mathrm{s}$ with $\mathrm{M}=2$, $8 \mathrm{Mbits} / \mathrm{s}$ with $\mathrm{M}=4$ and $13.2 \mathrm{Mbits} / \mathrm{s}$ with $M=6$. Increasing $M$ beyond 6 results in a very small and eventually negligible increment in maximum data rate. It should also be pointed out that these results were obtained by assuming fixed and arbitrary sampling time. Since the maximal ratio combiner weights and the output SNR both vary with sampling time in a frequency-selective channel, an ideal maximal ratio combiner would determine both the best sampling time and the weights for that sampling time and track both the best sampling time and weights. Hence maximal-ratio combining can be quite complex in wideband systems.

14 This corresponds to a symbol rate of $2 \mathrm{Mbits} / \mathrm{s}$ for a typical mean ms delay spread of $25 \mathrm{~ns}$ or 1 Mbits/s for a typical peak rms delay spread of 50 ns.

15 This value is based on $0 \%$ roll-ofr SRC pulses and represents an average value for different diversity orders $(2,4,6$ and 8$)$ of maximal ratio combining [102]. 
Selection diversity is not as complex and Valenzuela [124] studied its performance for coherently detected 4-QAM and a rms delay spread of 25 ns. Increasing the diversity order $M$ from 1 to 2 reduced the outage probability (for a BER of $10^{-4}$ ) from 0.012 to 0.0004 at $2 \mathrm{Mbits} / \mathrm{s}$ and from 0.15 to 0.05 at $8 \mathrm{Mbits} / \mathrm{s}$. A comparison with the results of Winters and Yeh for coherently detected QPSK with maximal-ratio combining of order $\mathrm{M}=2$ and $\mathrm{ms}$ delay spread of $25 \mathrm{~ns}$ yields a maximum data rate of $5.5 \mathrm{Mbits} / \mathrm{s}$. It can thus be concluded that one must accept at least a $50 \%$ to $70 \%$ reduction in data rate when using selection diversity instead of maximal-ratio combining.

\subsubsection{Equalization}

It thus becomes clear that, if data rates of $5 \mathrm{Mbits} / \mathrm{s}$ or $10 \mathrm{Mbits} / \mathrm{s}$ (or more) are to achieved with a reasonable order (i.e. 2) of space diversity, equalization will be necessary to cancel intersymbol interference. In contrast to the vehicular mobile radio application [125], the use of an equalizer structure for wideband indoor wireless communications constitutes a significant increase in system complexity, particularly for the hand-held portables. It is therefore important to carefully select the equalizer in order to obtain desired performance while limiting the growth in complexity. Important criteria are the ability to equalize deep fades, small sensitivity to sampling phase to reduce the effects of timing jitter caused by multipath [126] and the ability to track the dynamic fading process. The latter is however a lesser concern for indoor communications in view of the quasi-static nature of the channel.

Monsen has shown [127, 128, 129] that a decision feedback equalizer (DFE) yields performance superior to that of a linear equalizer on a slow fading channel under the assumption of correct previous decisions. This results from the fact that the DFE's forward filter mitigates only precursor ISI, i.e. ISI due to symbols for which decisions have yet to be made. The backward filter operating on the reconstructed data sequence thus acts to eliminate the postcursor ISI. Since, in the absence of decision errors, this can be done exactly with no noise enhancement, it follows that the DFE will exhibit superior ability to equalize deep fades given this premise. Under a minimum meansquare error criterion (MMSE), this has been established mathematically [127]. The superiority of the nonlinear structure has also been noted by Sari [130] and Lecler and 
Vandamme [131] who found that a DFE with a T-spaced (i.e. symbol-spaced) 6-tap forward fiter could provide satisfactory performance without the help of space diversity on a $140 \mathrm{Mbits} / \mathrm{s}$ microwave digital radio link employing 16 QAM modulation.

The sensitivity to sampling phase tends to increase with excess bandwidth. For an infinite $T$-spaced linear equalizer, it has been shown [132] that the excess bandwidth must be down to $1 \%$ or $2 \%$ before the effect of sampling phase becomes insignificant. The sensitivity to sampling phase can also be reduced by spacing the forward filter tap; by a fraction, most often $1 / 2$, of the symbol interval $T$. This property of fractionallyspaced equalizers (FSE) $[133,134]$ can be explained heuristically by noting that variations in the sampling phase have a much smaller effect on the amplitude and delay characteristics in the spectral overlap region of the sampled equalizer input ${ }^{16}$ when the tap spacing is smaller than $T$. This effect has been analyzed and quantified by Vandamme $e t$ al. [135] who showed that the mean square error sensitivity to the timing phase, though important for T-spaced equalizers, is a very fast decaying function of the fractional tap spacing; even for tap spacings close to $T$ (e.g. 0.9T), the MSE corresponding to the worse sampling phase was found to be far smaller than the corresponding value for a tap spacing of $T$. A number of comparative studies of fractionaily-spaced and $T$-spaced equalizers have also been carried out in the context of $140 \mathrm{Mbits} / \mathrm{s}$, high-level QAM, digital microwsve radio links [136, 137, 138]. For this channel, it was shown that the FSE can achieve decreased sensitivity to timing phase without compromising performance and with the same number of taps as the $T$. spaced -qualizer. Another advantage of fractional tap spacing results from the ability of an (infinite length) FSE to synthesize $[133,134]$ the optimum linear receiver for a digital sequence corrupted by ISI and AWGN. It is well known [139] that this receiver consists of the cascade of a filter matched to the actual channel with a transversal T-spaced equalizer. Therefore, a matched filter can be omitted with an FSE without sacrificing performance providing the number of taps is sufficiently large.

16 As a tap spacing smaller than $T$ reduces the spectral overlap (aliasing) in the sampled equalizer input's spectrum, nulls are thus less likely to occur in the spectrum which makes it casier (with less noise enhancement) for the equalizer to make the spectrum flat. 
Given the advantage of the DFE in terms of its ability to equalize deep fades and of the FSE in terms of its sampling phase sensitivity ${ }^{17}$, Monsen [50] and Gitlin and Weinstein [133] combined the two, i.e. they proposed a FSE-DFE with fractional (T/2) forward filter tap spacing and a $T$-spaced backwand filter. These works dealt with the tropospheric scattering and telephone channels respectively and only static performance was considered. Brunner and Weaver [141] later determined that this FSE-DFE structure has worse tracking ability than the DFE with the LMS (least meansquare) algorithm used to update the taps. In very fast fading, the RLS (recursive leastsquares) algorithm [142, 143] could be used to alleviate this effect. However, in the quasi-static indoor medium, LMS adaptation should provide satisfactory performance.

For all these equalizer structures, as long as the total delay spread is no larger than the forward filter length (number of taps * tap spacing), the resulting performance is found [127] to be superior to the flat fading case. This phenomenon is due to the equalizer's ability to resolve effectively the uncorrelated multiple paths and thus to take advantage of the implicit time diversity of the channel. It should be noted that one could choose some other criterion than MMSE for tap adjustment and arrive at a similar solution for the optimum tap weights. In fact, Baird and Zahm [51] have examined the more general problem of optimizing the tap weights of a space diversity predetection combiner under various performance criteria such as maximum signal-to-noise ratio and MMSE. It was found that the optimum solutions for the tap values differ only by a scalar gain factor, independent of the tap weights. In this sense, an equalizer can be considered as a diversity combiner with an implicit diversity order proportional to the number of forward filter taps. The diversity order can also be increased by adding explicit space diversity to the equalizer structure as in [50] where $M$ forward filters (each with $\mathrm{L}$ taps) are used to provide an effective diversity orier of $M * L$. The equivalence of the optimum tap weight solutions under MMSE and maximum SNR criteria was also established [50] specifically for this case.

17 It should be noted that a DFE with a T-spaced forward filter is also less sensitive to sampling phase variations than its linear T-spaced counterpart [140]. 
It should be noted that Monsen's paper [50] refers to "noise" as the combination of additive white gaussian noise and intersymbol interference. If "noise" is used to refer, as will be the case throughout this thesis, to AWGN only i.e. to impairments which are uncorrelated even in the short-term across both the explicit and implicit diversity branches ${ }^{18}$, Monsen's multiple forward filter equalizer effectively maximizes the signal-to-interference-plus-noise ratio (SINR). It implements a form of MMSE or optimum baseband combining as it not only provides protection against fading but also cancels interference by exploiting its short-term correlation across both the explicit and implicit branches. It is thus superior to maximal-ratio combining ${ }^{19}$ which, by maximizing the signal-to-noise ratio, only provides fading protection and considers all impairments as (both short- and long-term) uncorrelated across the diversity branches. It should also be noted that a sequence estimation technique, i.e. making decisions on sequences of symbols, could be made optimum in terms of probability of error (maximum-likelihood detector) and thus superior to this structure which, although it processes several symbols at a time, only makes decisions on a per-symbol basis. More on this in Section 2.3.2 dealing with cochannel interference.

In recent years: a few studies specifically geared to the use of adaptive equalizer schemes for indoor wireless communications have been performed. Valenzuela [145] has evaluated the potential performance improvements resulting from the use of adaptive equalization in a 4-QAM indoor radio link with coherent detection. Two theoretical bounds were considered. The MMSE equalizer bound [146] representing the optimum linear processor can be implemented in practice with a transversal filter with enough fractionally-spaced taps to achieve the desired response. The matched filter bound [146] representing the optimal nonlinear processor is obtained by assuming that one can detect each symbol optimally by a matched filter without incurring the penalty of ISI. Clearly, it is impossible for any equalizer to perform better than this fictional system but a DFE can be used to approach this goal in practice. With the MMSE

18 The explicit and implicit diversity bra. hes respectively provide a form of space and time diversity.

19 Maximal-ratio combining can reduce ISI [144, 79] if the diversity order is very large (i.e 6 or more). However, this is a marginal effect where the ISI from each branch tends to add with random phase. as with noise. 
bound, Valenzuela determined that, for an outage rate of $10^{-3}$ at a BER of $10^{-4}$, rates of up to $4 \mathrm{Mbits} / \mathrm{s}$ could be supported in buildings with rms delay spreads of $25 \mathrm{~ns}$. The ultimate performance limit of the matched filter bound suggests that rates of up to $64 \mathrm{Mbits} / \mathrm{s}$ could be approached under these conditions. In addition, performance analysis of a DFE based on a gaussian assumption of ISI and on an exponential delay profile [94] or on profiles [147] measured in [148], showed that signaling rates of 10 Mbits/s were obtainable with three forward filter taps. Hence, the use of postdetection diversity combining in the form of an equalizer could significandly increase achievable data rates in a wideband TDMA indoor wireless indoor communications system.

\subsubsection{Coding}

Channel coding provides another form of diversity in which the signal components carrying the same information are separated in time for bandwidth-expanding block and convolutional codes or are part of an expanded constellation set in the case of trellis codes. For example, trellis codes have been the subject of numerous investigations for use on fiat fading mobile satellite channels [149] and very recently in combination with space diversity (equal-gain combining) on flat fading indoor channels with cochannel interference [150] or in conjunction with a linear equalization on frequency-selective indoor channels corrupted strictly by AWGN (i.e. no cochannel interference)[151]. Although they avoid bandwidth expansion, trellis codes were found [150] to be far more sensitive to cochannel inierference than bandwidth-expanding convolutional codes in view of a more crowded constellation set. This is particularly true in the absence of interleaving [150] which, as will be discussed shortly, may not be practical on the indoor channel. Hence, trellis codes will not be considered for the frequency-selective fading application considered in this thesis as the performance of constellation sets ${ }^{20}$ with a large number of levels degrades substantially in highly dispersive multipath environments [152]. Block codes for fading channel applications are often selected [153] from a large sub-class of cyclic codes known as Bose-Chaudhuri-Hocquenghem

20 The relatively small trellis code constellation set of 8PSK used in conjunction with a three-tap lincar equalizer in [151] yielded acceptable performance even with no interleaving over an AWGN corrupled frequency-selective indoor channel. It remains to be seen how bandwidth-expanding codes compare in this situation and particularly in the presence of cochannel interference. 
( $\mathrm{BCH})$ codes [154]; however, long binary $\mathrm{BCH}$ codes tend to have poor distance properties, i.e. as the block length $n$ of these codes increases, their normalized minimum distance $\frac{d_{\text {min }}}{n}$ quickly approaches zero [71]. For this reason, a nonbinary sub-class of $\mathrm{BCH}$ codes with superior minimum distance characteristics, th: Reed-Solomon (RS) codes $[154,71,155,156]$ are most popular to combat random and bursty errors on fading channels. Both hard-decision (e.g. the Berlekamp-Massey-Forney algorithm [154]) and various simplified soft-decision decoding algorithms [157] have made it possible to implement such codes with relatively long block lengths. On the other hand, convolutional codes with soft-decision Viterbi decoding [158] have long been known to outperform block codes of the same order of complexity both on the simple memoryless AWGN channel [159] and on fading channels [58] where, in addition, channel state information in the form of the instantaneous fading depth, can be incorporated in the Viterbi decoder metric [160] to enhance performinace even more.

On fading channels, these coding techniques are generally employed in conjunction with symbol interleaving [161] in order to disperse burst errors to which the Viterbi decoder, in particular, is very sensitive. The simples: interleaving method is referred to as block iriterleaving where the encoded symbols are written into memory on a row-byrow basis and then read out on a column-by-column basis. Niore complex interleaving schemes, such as convolutional interleaving [162], can reduce storage costs and provide shorter delay which should not exceed 30 to $60 \mathrm{~ms}$ for voice communication. This constraint imposes an upper limit on the interleaving depth which should be of the same order as the coherence time of the channel in order to effectively randomize errors. Krishnamurthi et al. [163] have shown that, for a block-coded coherent BPSK transceiver operating on a slowly-varying isuloor flat fading channel with a mean fade duration of $60 \mathrm{~ms}, \mathrm{BCH}$ codes with the maximum acceptable (in terms of delay) interleaving depth, could provide significant performance gains over the uncoded case. However, these gains vanished when no interleaving was used. More recently, it also has been argued [164] that, for a given bandwidth allocation and capacity requirement in a cellular frequency-reuse system, the lower radio link signal-to-interference ratio (SIR) resulting from the bandwidth expansion makes convolutional coding with little or 
no interleaving unattractive in terms of spectral efficiency. Mean fade durations of 60 ms are typical for a walking user but for stationary terminals, fades can be far longer. In this case, the interleaving depth would have to $b$, increased substantially to yield a coding gain. Coupled with the increased storage costs required when signaling in the Mbits/s range, this implies that interleaving may not be practical on the wideband indoor channel. To overcome this problem, Saleh et al.[122, 80] proposed using slow frequency hopping to randomize errors in an indoor radio system employing two-stage Reed-Solomon coding. Variable coding rate schemes $[165]^{21}$, i.e. employing punctured codes, have also been studied to alleviate the effects of very long fades for which the channel can only be partially interleaved ${ }^{22}$. However, particularly for stationary terminals, the latter scheme could involve a significant throughput penalty whereas the frequency hopping scheme can, as discussed earlier, require large bandwidth for decorrelation in view of the small delay spreads in an indoor environment.

Given that interleaving cannot be used to randomize errors on the wideband indoor radio channel, coding gains can still be achieved by combining space diversity with coding. In general, coding provides much larger gains than conventional space diversity for "good" channels but suffers from a threshold effect [168] in the sense that the coding gain can vanish completely when the channel becomes too "poor". Hence, with diversity, the decoder can see an improved channel and can make better use of the code redundancy in order to yield positive coding gains. Miyagaki et al. [56] have performed a detailed study of the compound effect of block binary $(n, k, t) \mathrm{BCH}^{23}$ coding, interleaving and maximal-ratio combining on the performance of noncoherent FSK on a Rayleigh fading channel with a fading rate of $20 \mathrm{~Hz}$. The average bit error rate performance of nine different binary $\mathrm{BCH}$ codes with error correction capability $t$ of 1, 2 or 3 was compared ior various onders of space diversity. Miyagaki et al. [56]'s

21 This paper dealt with land mobile radio channel but the punctured code technique could in principle also be applied to the indoor medium.

22 Performance degradations due to partial interleaving have been assessed via analytical bounds [166] and by simulation [167]. The latter work has shown that RS block codes are slightly less sensitive to partial interleaving than convolutional codes of comparable complexity.

23 The parameter $t$ denotes the code's error correction capability. The binary BCH code's minimum distince is then $2 t+1[154]$. 
paper is in Japanese but some conclusions can be drawn from the English-language Figure captions. In particular, it was found that

1. With no space diversity, a non-inierleaved coded system performs worse (asymptotically) than the corresponding uncoded system except for very powerful (long block length and large minimum distance) codes. However, with a diversity order of 2 , the non-interleaved coded system can match or outperform the corresponding uncoded system.

2. A dual diversity system with no interleaving can outperform ${ }^{24}$ a single branch diversity system with nearly "full" interleaving, i.e. for which an increase in interleaving depth yields a negligible performance gain.

3. For the $t=1$ and the $t=2$ codes, the non-interleaved coded dual diversity system could not outperform the uncoded triple diversity system. To do so, a code with minimum distance corresponding to $t \geq 3$ is required. On the other hand, with full interleaving, a coded dual diversity system yielded significant performance gains over the uncoded triple diversity system for codes with $t=2$ and over the uncoded quadruple diversity system for codes with $t=3$.

Hence, it appears that for a given diversity order, space diversity can help to produce positive coding gains on a non-interleaved fading channel. By using codes with large block length and minimum distance, it also seems that, even without interleaving, channel coding could provide a viable alternative to increasing the space diversity order providing the latter is already larger than 1 . Of course, both the fading channel characteristics and the transceiver structure in Miyagaki et al.'s work are different than in the wideband indoor radio context. It remains to be seen if these conclusions could also apply in this case and for convolutional codes with Viterbi decoding. In addition, since adaptive equalization is required in view of the high signaling rates considered in this thesis, the effective diversity order will be far larger than in Miyagaki et a: $[56\}$ and this will probably influence the choice between increasing the explicit space diversity order of a optimum baseband combiner or using channel coding. It should be noted

24 This is true asymptotically and at most error rates of interest except at low $\frac{E_{4}}{N_{11}}$ for codes with $t=3$, i.e. with langer minimum distance. 
that, to the author's knowledge, no study of the compound effect of channel coding (with and without interleaving), decision feedback equalization and space diversity has been undertaken for the indoor portable or vehicular mobile radio channels. Cleverly et al. [169] have studied the performance of Reed-Solomon coding with a DFE for different interleaving depths on a rapidly-varying fading channel. Another recent paper by Uesugi et al. [170] has dealt with using convolutional codes (with Viterbi decoding) in conjunction with a DFE on the vehicular mobile radio channel but with a fixed interleaving depth and again without consideration of space diversity.

\subsubsection{Techniques to combat cochannel interference}

The problem of interference from other users ${ }^{25}$ can be reduced with techniques such as dynamic channel assignment [121] where assigned channels are continuously monitored for interference and the assignment is changed to a quiet channel when the interference becomes too strong or by transmit power control [121] to alleviate the large signal fluctuations due to shadowing. Space diversity can also be used to suppress interference and enhance desired signal reception via (predetection) optimum combining [53] whereby the signals received by multiple antennas are weighted and combined to maximize the signal-to-interference-plus-noise ratio (SINR) at the antenna array output. Such adaptive arrays have been studied extensively [171] ior non-fading channels and also for fading channels first by Bogachev and Kiselev [172] who provided analysis for the case of a single (gaussian) interferer and later via simulation by Winters [53]. Over fading channels, the advantage of optimum combining is based on the fact that, over a period smaller than the average fade duration (which is at least $10^{5}$ symbols when signaling in the Mbits/s range in the indoor medium), the interfering signals at the various receiver antennas are strongly correlated, even though they are uncorrelated in the long term, i.e. over a large number of fading channel realizations. This shortterm correlation can thus be exploited to achieve a higher SINR than maximal-ratio combining which assumes that all interference and noise are uncorrelated. Hence, the larger the proportion of the total interference and AWGN power occupied by the

25 As discussed earlier, the techniques discussed in this Section can be used to alleviate the effects of not only cochannel interference but also adjacent channel interference. 
interference, the larger the improvement of optimum combining will be over maximalratio combining. Optimum combining can be implemented either at the base station [53] or at the mobile [173] and adaptive retransmission with time division ${ }^{26}[174]$ can be used to obtain the same performance in both transmission directions with only one antenna at the other end of the radio link. In the same way as an equalizer, adaptation of the tap at each antenna can be performed with the LMS algorithm 1143]; although this algorithm takes longer to converge than some other algorithms, it is simple to implement and, for the slow fading of indoor radio, should still be able to track the desired and interfering signals as the propagation characteristics of the channel vary. The desired and interfering signals can be distinguished at the receiver by different pseudo-random codes [53]. Computer simulations of $\mathrm{M}$-antenna optimum combining in indoor radio [52] have shown that, based on a $10^{-2}$ outage probability at a $10^{-3} \mathrm{BER}$, up to $\mathrm{M}-\mathrm{I}$ interferers of large power could be tolerated provided the desired signal-to-noise ratio (SNR) was large enough. Recent works have also confirmed the superiority of optimum combining over other combining techniques for both the vehicular mobile radio [144] and indoor [175] environments.

The aforementioned studies of optimum combining all assumed a flat fading channel. However when the channel exhibits significant time dispersion, the optimum weights vary with the sampling time just as with maximal-ratio combining. Hence, at high data rates, implementation of predetection optimum combining becomes a multidimensional optimization problem which is probably too complex to be practical. As equalization is necessary in this case, it is worthwhile to consider the optimum baseband combiner discussed earlier as a means of simultaneously combatting signal fading and interference. In fact, both linear and decision-feedback equalizers have long been known to reject interference $[176,177,118]$. This can be understood by noting that from the point of view of receiver optimization, cochannel (as well as adjacent channel) interference can be treated [178] as a generalization of intersymbol

26 This involves multiplying the signal transmitted by each antenna by the magnitude of the receive weight for that antenna, but phase shifted by the negative of the receive weight, i.e. phase lag becomes phase lead and vice-versa. In this way, the transmit antenna pattem is the same as the receive patlem. 
interference. When an equalizer is used in conjunction with space diversity, i.e. by using multiple transversal filters with possibly a common feedback filter, cochannel irterference correlated between the various diversity branches can then be cancelled [49], thus maximizing the output signal-to-interference-plus-noise ratio (SINR). Of course, optimum baseband combining requires multiple antennas at both ends of the radio link and cannot tolerate high power interferers (i.e. stronger than the desired signal) unless some form of null steering is applied as in digital microwave radio links [49]. However as discussed earlier, in cellular mobile and portable radio systems, macroscopic diversity can be used to alleviate the effect of local peaks in the power of a cochannel interferer. In addition, the implicit diversity gain obtained from the combining of the uncorrelated multipath returns tends to offset the sacrifice of explicit diversity which is required to cancel interference, a feature not available with predetection optimum combining $[52,53]$ where for a given number of antennas, the effective diversity order is much smaller. Therefore, providing that macroscopic diversity is used to take care of high power interferers, optimum baseband combining is an attractive microscopic diversity technique for radio links in a wideband TDMA indoor communication system.

\subsection{Capacity issues}

The number of users that can simultaneously be handled in a frequency-reuse radio communication system is highly dependent on the radio links' capacity or maximum bit rate per unit of bandwidth for which error-free digital communication can theoretically be achieved. In 1949, Shannon [179]showed that the channel capacity $C$ of a bandlimited white gaussian noise channel is

$$
C=B \log _{2}\left(1+\frac{S}{N}\right) \quad \text { bits } / \text { sec } / H z
$$

where $B$ is the channel bandwidth, $S$ is the RF signal power and $N$ is the noise power within the bandwidth. It can be seen that the channel capacity goes to infinity when $\stackrel{S}{N}$ goes to infinity. However, the capacity remains finite even for infinite bandwidth as the noise power also increases with an increase in bandwidth. 
In a (flat) Rayleigh fading environment with gaussian noise, the channel capacity can be determined in an average sense as $\frac{S}{N}$ is no longer a constant but a variable following the fading statistics. It has been determined by Lee [180] that, for any value of $\frac{S}{N}$, the channel capacity in a Rayleigh fading environment is always lower than the capacity in a gaussian noise channel and the discrepancy increases as $\frac{S}{N}$ is reduced. However, by using M-branch antenna diversity with maximal-ratio combining ${ }^{27}$, the capacity on the Rayleigh fading channel approaches the capacity in gaussian noise as $M \rightarrow \infty$. For example, at $\frac{S}{N}=15 d B$, the capacity in gaussian noise is $5 \mathrm{bits} / \mathrm{s} / \mathrm{Hz}$. whereas in Rayleigh fading, it is 4 bits/s/Hz with $M=1,4.7$ bits/s/Hz with $M=2$, and $4.9 \mathrm{bits} / \mathrm{s} / \mathrm{Hz}$ with $\mathrm{M}=4$ [180]. This shows that a small order of space diversity can be an effective tool to mitiga'e the effects of signal fading on the maximum data rate of a fading radio link.

A more detailed study of the fundamental limits on the data rate of multiple antenna systems in a flat Rayleigh fading environment was performed by Winters [181]. The distribution of the efficiency index, i.e. the maximum data rate for a given average error rate, for both optimum linear and nonlinear processing ${ }^{28}$, was obtained for two radio link configurations applicable to the mobile and/or portable environments namely, 1) $N$ remotes each with one antenna transmitting to a base station with $M$ antennas and 2) communication between two users, each with $\mathbf{M}$ antennas. In both cases, results show that, based on a $10^{-3}$ outage probability at a $10^{-4}$ error rate and with optimum linear processing at the receiver, up to $M / 2$ channels can be established with approximately the same data rate as a single channel. With either nonlinear processing at the receiver in case 1 or optimum linear transmitter/receiver processing in case 2, up to $M$ channels can now be established with approximately the same data rate as a single channel. For both cases, the capacity bounds show the potential for high rate communication in bandwidth-limited situations. For example, in case 2 with optimum linear Tx/Rx processing, the maximum data rate (for a $10^{-3}$ outage probability at a $10^{-4}$ error rate)

27 As discussed earlier, this is the best combining technique when the only channel impairments are flat Rayleigh fading and white gaussian noise.

28 The optimum linear processor is considered optimum in the sense of minimizing mean square error. The optimum nonlinear processor minimizes probability of error (maximum likelihood detector) [181]. 
at a given SNR $\rho$ is the same as the capacity at $\rho-8.2 \mathrm{~dB}$. This implies that large gains could be achieved by using channel coding.

The analytical basis for Winters' work [181] is found in a series of papers [182, 183,184 ] dealing with theoretical performance bounds for digital communications over frequency-selective fading channels and particularly, digital microwave radio. In [182], the performance of linear and decision feedback equalization as well as maximum likelihood sequence estimation, is analyzed for the case of a single channel (i.e. $\mathrm{N}=\mathrm{M}=1$ ) using the probability distribution of the efficiency index as the performance measure. In [183], the analysis of [182] is extended to digital radio with dual polarization ( $\mathrm{N}=\mathrm{M}=2)$. The structure of the optimum transmitter and receiver matrix filters is studied, and the information-theoretic capacity and efficiency index with these filters is determined. The results of [183] are extended to a $N \times N$ channel matrix in [184] and to a $M \times N$ channel matrix in [185]. Hence, theoretical performance and capacity bounds have been obtained for frequency-selective fading radio channels. In [183] for example, it was shown that the combined channels' data rate associated with an optimum nonlinear technique differs from the capacity bound by at least 3 bits/sec/Hz which points to coding in order to recoup some of this loss.

It should be noted that the quantitative results of $[182,183,184]$ are all based on a channel model applicable to long distance terrestrial microwave radio links. This model uses a simple two-ray representation and is due to Rummler $[186,187]$ after measurements of a 26 mile hop in the $6 \mathrm{GHz}$ band. For wideband indoor radio channels, there is generally more than one significant reflected ray; hence, the influence of parameters such as delay spread and delay profile shape on channel capacity has not been evaluated. It may therefore be worthwhile, as done by Winters [181] in the flat fading case, to apply the analysis of $[182,183,184]$ to a model yielding a better representation of frequency-selective indoor radio channels.

\subsection{Summary}

In this Chapter, a survey of documents relevant to the characterization of the wideband TDMA indoor radio chunnel and to the various techniques available to counteract 
its impairments, has been performed. Optimum baseband combining, possibly used in conjunction with channel coding, was then proposed as an attractive technique to support high data rate indoor radio links, provided that it is used in conjunction with macroscopic diversity to alleviate severe shadowing effects. A survey of works dealing with channel capacity also showed that the effect of various wideband indoor channel parameters on such theoretical performance bounds has not been determined. 


\section{Chapter 3 \\ ANALYTICAL PERFORMANCE EVALUATION}

\subsection{Introduction}

Following a description of the system model, this Chapter discusses an analytical approach to evaluating the bit error rate performance of optimum baseband combining in conjunction with convolutional channel coding and soft-aecision Viterbi decoding on indoor radio links. For this purpose, previous (uncoded) optimum baseband combiner derivations [50] for tropospheric scatter channels and based on a non-fading gaussian characterization of interference are applied to the case of frequency-selective, Rayleigh-fading, indoor radio links employing BPSK or QPSK and impaired by cochannel interference. Novel average bit error rate bounds are then developed for the coded system under the assumption of ideal interleaving. Such an approach is advantageous as it quickly provides a large number of results without resorting to lengthy simulations. A comprehensive set of results is thus presented in terms of a variety of critical system parameters such as signal-to-interference ratio, receiver sampling phase, transmit/receive pulse shape, number of forward filter taps and signaling rate, i.e. delay spread normalized to the symbol interval. The latter two parameters strongly influence the system's ability to exploit the channel's implicit diversity effect. BPSK and QPSK modulations are also compared based on a given rate of information throughput as well as in both interference-dominated and white noise-dominated situations. The option of using convolutional channel coding with soft-decision Viterbi decoding as an altemative to increasing the explicit space diversity onder from two to three is also examined. 


\subsection{System description}

A functional block diagram of the system under study is shown in Figure 3.1. In this representation, $n$ indexes the portable $(n=0)$ whose signal the base station wishes to receive and the $N$ interfering portables $(n=1,2, \ldots, N)$ representing cochannel interference from other cells operating on the same frequency. Each portable's input data stream, which may be binary or complex binary (to represent four-phase signaling), is fed into a (bandwidth-expanding) convolutional channel coder producing the coded bit stream $\left\{s_{k}\right\}_{n}$. The transmitter output is

$$
s_{n}(t)=\sum_{k=-\infty}^{\infty} s_{k n} g(t-k \cdot T)
$$

where $T$ is the interval between successive symbol transmissions, i.e. symbol interval. The $\left\{s_{k}\right\}_{n}$ are infinitely long sequences of statistically independent binary or quat: rnary symbols selected from the set $(+/-1+/-j)$ for quatemary signaling or from the set $(+/-1)$ for binary signaling. The receive filter function is matched to the transmitter pulse shape $g(t)$; as well, their combination $f(t)=\int_{-\infty}^{\infty} g(t+u) g(u) d u$ is defined a unit energy real function and satisfies the Nyquist criterion for zero ISI at the sampling instant.

The list of modulation candidates can be narrowed down by noting that since adjacent channel energy can be reduced by distance separation, filtering and modulation spectrum shaping, it is not necessary to choose a constant envelope modulation scheme with extremely low far-out spectral energy (such as TFM or GMSK) for small coverage area, low-power, frequency-reuse digital portable communications. It is preferable to compromise more [1] on the side of conservation of portable set power and detection sensitivity at the expense of which a reduction in far-out spectral energy is usually accomplished. These low transmit power levels and the development of linear RF power amplifiers [188] thus make non-constant envelope schemes such as two- and four-level phase-shift keying (BPSK and QPSK) attractive candidates for the portable application. The same cannot be said of PSK modulation with a higher number of levels which requires less bandwidth but more power for a given error rate performance. In addition, even when compared on the basis of constant information throughput, these higher-level schemes such 8-PSK and 16-QAM produce relatively similar irreducible 
Portable $n(n=0,1, \ldots, N)$
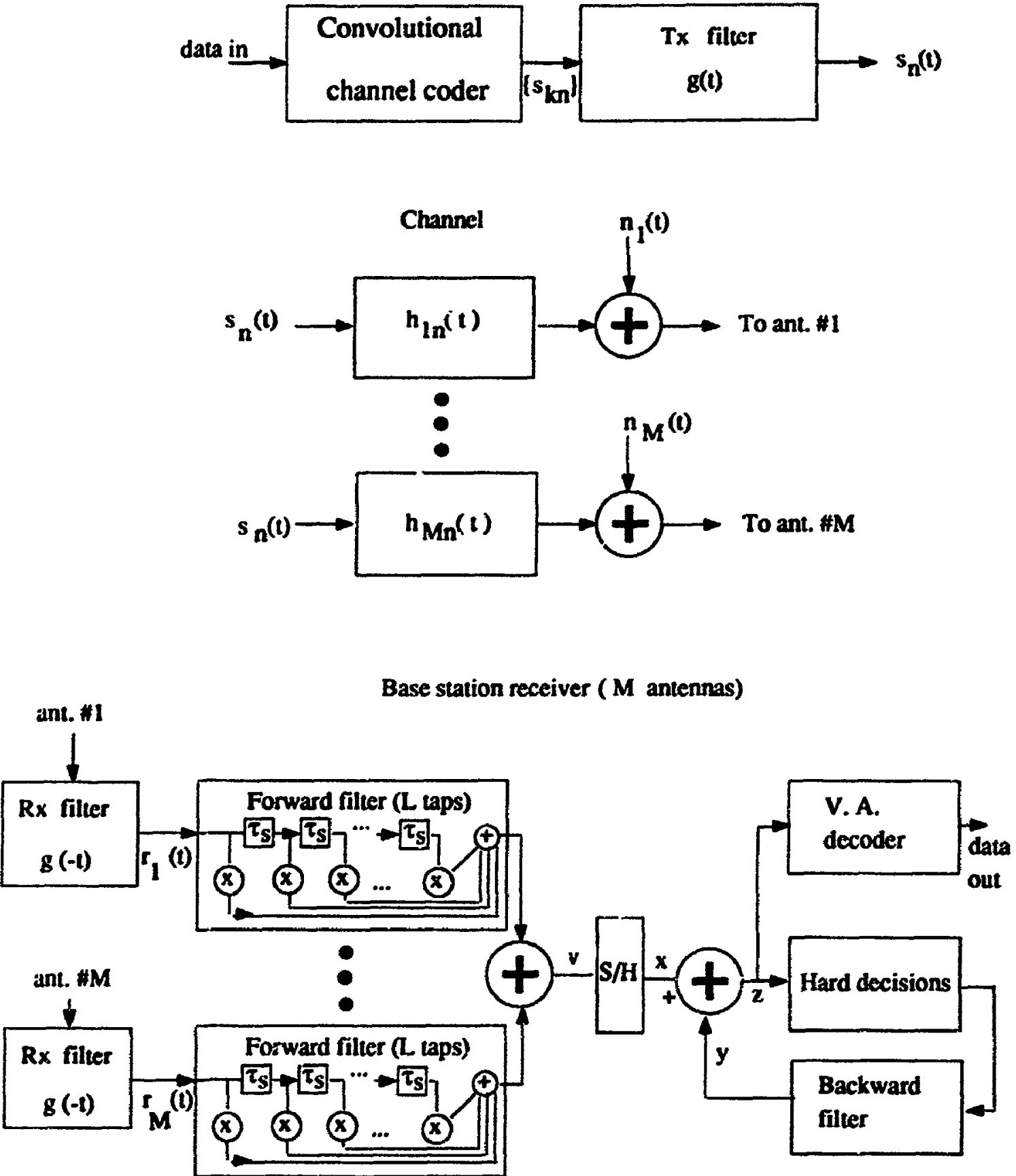

Figure 3.1 System model (low-pass equivalent). 
error rates to QPSK with pulse shapes of rolloff larger than $25 \%$ but much higher irreducible error rates than QPSK when the rolloff exceeds 25\% [189]. Coherent detection is also selected as it has been shown [190] to be about $2.4 \mathrm{~dB}$ more sensitive than differential detection in a typical portable radio channel where the slow portable-set movements result in slow channel phase variations (compared to conventional vehicular mobile) that the carrier recovery loop can follow. In addition, differential detection can be difficult to implement [191] in a receiver with equalization.

To evaluate the effect of frequency-selective fading caused by delay spread, the (Rayleigh) fading multipath channel between the $n^{\text {th }}$ portable $(n=0,1, \ldots, N)$ and the $i^{\text {th }}$ base station antenna $(i=1,2, \ldots, M)$ is characterized by an ensemble of zero-mean random functions with complex gaussian statistics, i.e.

$$
h_{n i}(t)=h_{c_{n t}}(t)+j h_{s_{n t}}(t)
$$

Although the various response functions of the ensemble, $h_{c_{n t}}(t)$ and $h_{s_{n t}}(t)$, change form as the portable moves (slowly) through the indoor radio environment, it is assumed that the impulse response does not change for time intervals corresponding to several thousand symbol periods. With coherence times upper bounded, as discussed in Chapter 2, by $100 \mathrm{~ms}$ and $250 \mathrm{~ms}$ for moving and stationary users respectively and with symbol periods of $1 \mu \mathrm{s}$ or less (i.e. signaling rites of $1 \mathrm{Msymbols} / \mathrm{s}$ or more), this assumption should be valid for both stationary and moving users respectively.

This quasi-static property of the various impulse functions implies they may be regarded as fixed functions taken from the ensemble and the actual time variations of these functions may be treated via the ensemble statistics. As discussed in Chapter 2 , each of the portable to base station antenna channel ensembles can be characterized by the mean-square magnitude or multipath delay profile of the impulse response functions. Specifically for the desired portable $(n=0)$ and the interfering portables $(n>0)$, one may write

$$
\begin{aligned}
\overline{h_{n i}(\tau) h_{m j}^{*}(\tau+x)} & = \begin{cases}\phi_{n i}(\tau) \delta(x) & \text { if } n=m \text { and } i=j \\
0 & \text { otherwise }\end{cases} \\
\text { where } \mathrm{n}, \mathrm{m} & =\mathbf{0}, 1, \ldots \mathrm{N} \text { and } \mathrm{i}, \mathrm{j}=1, \ldots \mathrm{M} .
\end{aligned}
$$


The overbar denotes averaging over the channel ensemble statistics and $\phi_{n i}(\tau)$ is the mean-square envelope of the impulse response or multipath delay profile between the $\mathrm{n}^{\text {th }}$ portable and the $\mathrm{i}^{\text {th }}$ base station antenna. The impulse responses are assumed to be scaled sich that the area of $\phi_{n i}(\tau)$ is unity for all $n$ and $i$. Note that equation 3.3 implies that the (Rayleigh) fading process is independent from one portable-to-base station antenna path to another.

Since the primary determinant of $\phi_{n i}(\tau)$ is the path geometry between the $\mathrm{n}^{\text {th }}$ portable and the $\mathrm{i}^{\text {th }}$ base station antenna, it follows that the delay profi's is virtually identical for all antennas for a given portable [79]. However, propagation geometries will differ from one portable to another and hence the delay profile will vary with $n$ accordingly. Nevertheless, to avoid complicating the analysis, a common average delay profile $\phi(r)$ is assumed for all $n$ and $i$. This assumption should not affect results to a great extent as previous studies [79] of diversity combining with cochannel inte:ference in frequency-selective fading have shown that the effect of the delay profile shape on system performance is negligible for ms delay spreads smaller than $50 \%$ of the symbol interval, i.e. for data rates of $20 \mathrm{Mbits} / \mathrm{s}$ or less with a typical $25 \mathrm{~ns}$ mean rms delay spread.

The complex additive white gaussian noise terms $n_{\mathfrak{t}}(t)$ are zero mean with second moments

$$
\mathrm{E}\left[n_{i}(t) n_{j}^{*}(t+r)\right]=N_{0} \hat{\delta}_{i j} \delta(\tau), \quad i, j=1,2, \ldots, M .
$$

where $N_{0}$ represents the single-sided white gaussian noise power spectral density (mean received noise power in one Hertz of bandwidth) over the frequency band of interest. The expectation operator $\mathrm{E}[\cdot]$ represents an average over the noise (or interfering or desired signal) statistics for a particular channel realization, i.e. for a given set of sample functions $h_{n i}(t)$ in a time interval smaller than the reciprocal of the fading rate (e.g. several thousand symbol periods). The analysis thus requires two different means $[52,49]$. As a res 1 t of the slow fading assumption, the equalizer can track the channel variations and from its viewpoint, the channel is locally time-invariant. The optimum DFE taps are a function of these (fixed) channel characteristics and the statistics of the noise and interfering signals. In the long term, the channel varies and 
the tap values as well as the resulting signal-to-interference-plus-noise ratio (SINR) are random variables with respect to the channel statistics. Hence, the short-term average $E[\cdot]$ will be referred to as local mean and the long-term average over the channel statistics $\overline{(T}$ ) simply as mean.

At the receiver, the demodulated signal in each space diversity branch is Scd to a forward filter whose detailed structure is shown in Figure 3.2 along with that of the feedback filter.

Each of these $M$ forward filters uses $L 1$ taps to reduce the effect of future digit intersymbol interference (i.e. precursors) from both the desired and interfering portables. They consist of a finite tapped delay line with tap spacing of $\tau_{\mathbf{s}}$ seconds and impulse response [50]

$$
u_{3}(t)=\sum_{l=-L_{l}}^{L_{2}} u_{\mathfrak{l}} \delta\left(t-l \tau_{s}\right) \quad i=1,2 . \ldots . M
$$

where the $L=L 1+L 2+1$ taps are split between $L 1$ "future" taps (operating on precursor ISI), L2 "past" taps (operating on postcursor ISI) and one center tap. As opposed to a structure using $L=L I+1$ forward filter taps, this structure, where $L 2$ forward filter taps operate in parallel with the backward filter, improves the rejection of cochannel interference [176] by processing postcursor samples in parallel with corresponding decisions. The sum of forward filter outputs $\nu$ is sampled at the symbol rate $1 / T$ and held for one symbol interval. The backward filter correction sample is then subtracted from the sample matched filter output to produce the detection variable $z$ which serves as input to the soft-decision Viterbi decoder. The signal-to-anterference-plus-noise ratio (SINR) at this point and its statistical distribution are thus crucial quantities for determining system performance.

In order to eliminate past digit intersymbol interference (i.e. postcursors), the backward filter weights previous decisions $\hat{s}_{k-1}$ to form the sample output $[50]$

$$
y=\sum_{i=1}^{B} b_{\imath} \hat{s}_{k-\imath}
$$

at the decision time for the $s_{k}$ symbol. The preliminary hard decision on $s_{k}$ is denoted as $\hat{s}_{k}$. In an uncoded system, the latter represents the symbol output. Note that with the 
$i^{\text {th }}$ FORWARD FILTER $(i=1, \ldots, M)$

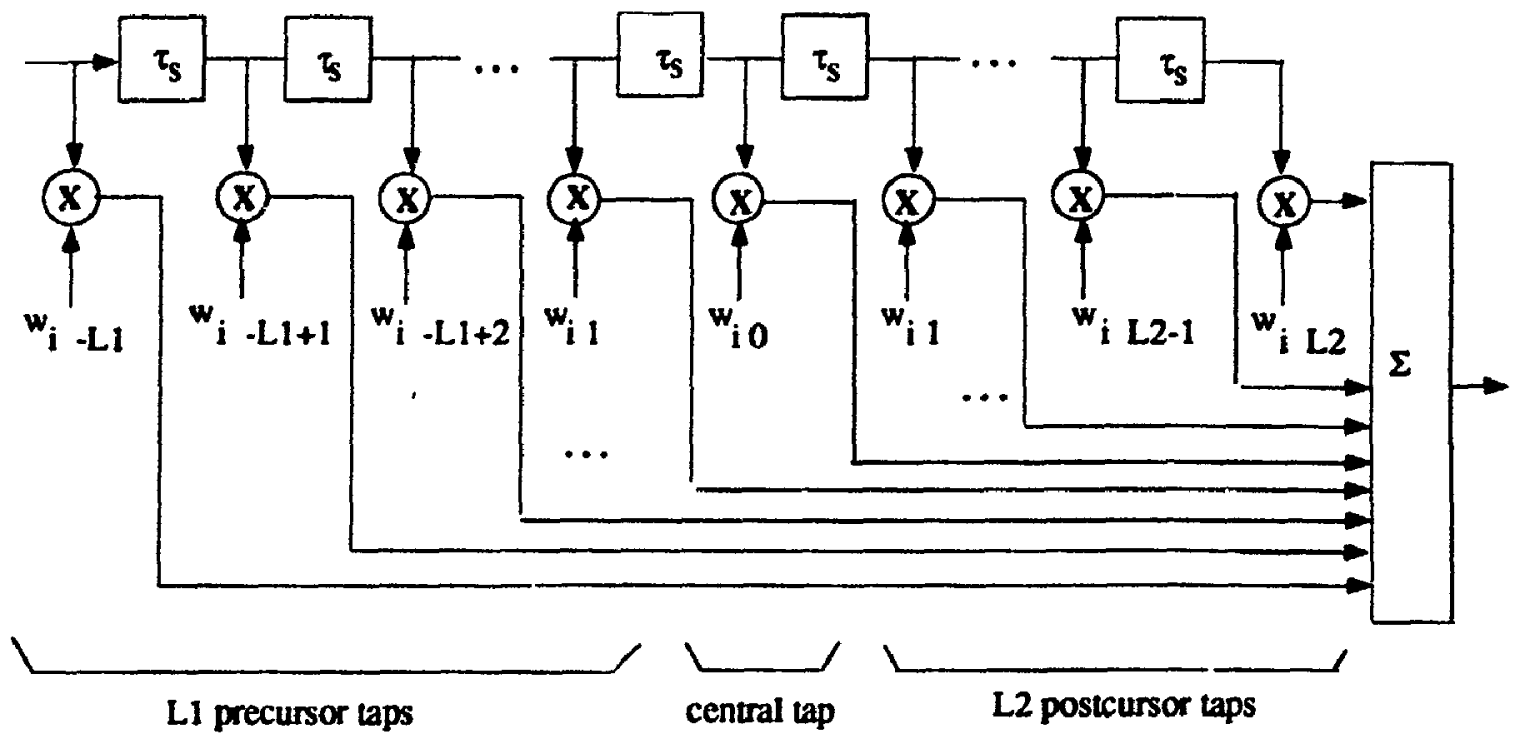

BACKWARD FILTER

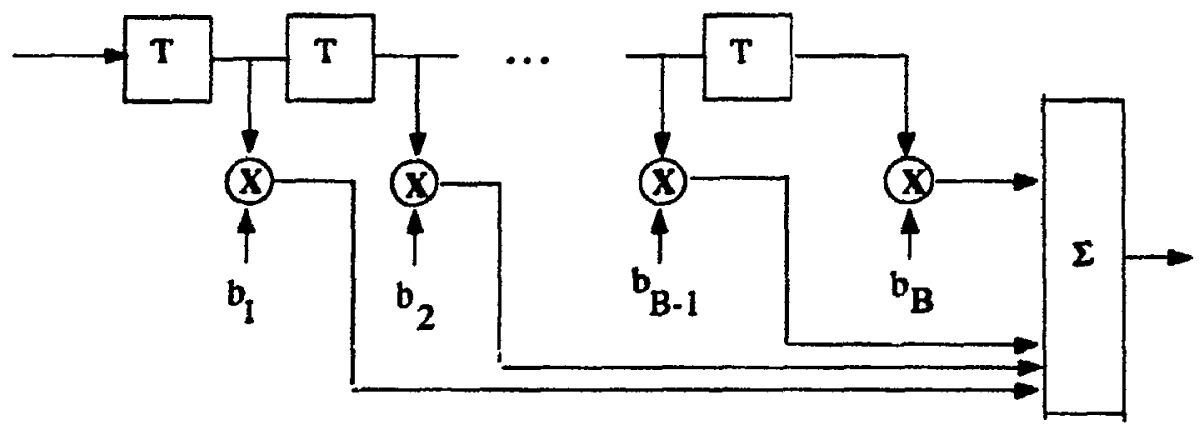

B feedback (postcursor) taps

Figure 3.2 MMSE decision feedback equalizer forward and feedback filters. 
slow time variations in the channel characteristics, all the forward and backward filter taps can be updated on a symbol-by-symbol basis by the LMS (least mean squares) algorithm [143].

\subsection{Determination of output SINR}

Initially, an expression for the set of optimum forward filter taps and the corresponding maximum SINR at the combiner output point $z$ in Figure 3.1 must be determined. Several parameters are involved and are defined as follows:

$S \triangleq$ mean received pou'er from desircd portable

$E_{S} \triangleq$ mean received energy per symbol from desired portable

$I_{n} \triangleq$ mean received pou'er from $n^{\text {th }}$ interfering portable

$E_{S n} \triangleq$ mean received energy per symbol

from $n^{\text {th }}$ interfering portable for $n=1, \ldots . N$.

$N_{p} \triangleq$ mean received noise power per antenna

$N_{0} \triangleq$ single - sided noise power spectral density

It is assumed tirat the front-end receive filter has approximately the same bandwidth as the transmitted (desired signal) which, for multiphase signaling, is simply the bandwidth [71] of the equivalent low-pass signal pulse $f(t)$. Expressing this bandwidth as $\frac{A}{T}$ where $A$ is a function of the excess bandwidth (i.e. $A=1$ for $0 \%$ rolloff, $A=1.5$ for $50 \%$ rolloff) and where $T$ is the symbol interval, the noise power and noise power spectral density are simply related by

$$
N_{p}=N_{0}\left(\frac{A}{T}\right)
$$

whereas the portable power and energy per symbol are

$$
\begin{aligned}
& S=E_{s}\left(\frac{1}{T}\right) \\
& I_{n}=E_{S n}\left(\frac{1}{T}\right) \quad \text { for } n=1, \ldots, N .
\end{aligned}
$$

where the energy per bit $E_{b}$ is equal to the energy per symbol $E_{s}$ for BPSK and equal to $\frac{E_{a}}{2}$ for QPSK. It should be noted that the different values of $S$ and $I_{n}, n=1, \ldots N$ (or 
of $E_{s}$ and $E_{S_{n}}$ ) are meant to represent large scale signal variations due to shadowing. In practice, these mean values are time-varying. However, they are herein assumed fixed as large scale signal variations are handled by macroscopic diversity and the primary goal of this work is to determine the small scale tolerance of a microscopic diversity radio link communication technique to given levels of cochannel interference induced partly by shadowing.

With these definitions, the mean SINR $\Gamma$ is now defined as an energy ratio, i.e.

$$
\Gamma=\frac{E_{s}}{N_{0}+\sum_{n=1}^{N} E_{S n}}=\frac{\left(\frac{E_{S}}{N_{i 1}}\right)}{1+\sum_{n=1}^{N}\left(\frac{E_{S_{n}}}{N_{11}}\right)}
$$

Since $\frac{E_{n n}}{N_{11}}=\left(\frac{E_{n}}{E_{s}}\right)\left(\frac{E_{2}}{N_{11}}\right)=\left(\frac{I_{n}}{S}\right)\left(\frac{E_{2}}{N_{11}}\right)$, this mean SINR can also be written as

$$
\Gamma=\frac{1}{\frac{N_{0}}{E_{s}}+\sum_{n=1}^{N} \frac{E_{S_{n}}}{E_{s}}}=\frac{1}{\frac{N_{n}}{E_{0}}+\sum_{n=1}^{N} \frac{l_{n}}{S}}
$$

where $\frac{L}{S}$ is the mean $n^{\text {th }}$ interferer to carrier power ratio. Note that, instead of an energy ratio, the mean SINR could also be defined as a power ratio in the form $S /\left[N_{p}+\sum_{n=1}^{N} I_{n}\right]$. The two definitions are equivalent only if the signal bandwidth is $1 / T$, i.e. if $A=1$ which is the case for a $0 \%$ SRC pulse. The definition of SINR as an energy ratio (equations 3.10 and 3.11 ) will however be used throughout this thesis.

The signals at the receiver forward filter inputs are ${ }^{29}$ :

$$
\begin{gathered}
r_{\imath}(t)=\sum_{k=-\infty}^{\infty} \int_{-\infty}^{\infty} s_{k 0} h_{0 i}(u) f(t-k T-u) d u \\
+\sum_{n=1}^{N} \sqrt{\frac{I_{n}}{S}} \sum_{k=-\infty}^{\infty} \int_{-\infty}^{\infty} s_{k n} h_{n i}(u) f\left(t-t_{n}-k T-u\right) d u \\
+\nu_{i}(t) \\
\text { where } n=1,2, \ldots, N \\
\text { and } i=1,2, \ldots, M .
\end{gathered}
$$

29 The $\sqrt{\frac{1}{5}}$ is used to account for different interferer power levels as all channel responses are normalized to unit energy. 
where

$$
\nu_{u}(t)=\int_{-\infty}^{\infty} n_{1}(t-u) g(u) d u \quad i=1, \ldots . . M
$$

and where $t_{n}$ represents the fixed difference in timing phase between the $n^{\text {th }}$ interfering portable $(n=1,2, \ldots, N)$ and the desired portable $(n=0)$. The more realistic case of random interferer timing phases ${ }^{30}$ will be considered in Chapter 5 . The convolution of the combined transmit/receive pulse shape with each of the $(N+1)^{*} M$ channel realizations is defined as:

$$
\begin{gathered}
q_{0 i}(t)=\int_{-\infty}^{\infty} f(t-u) h_{0 i}(u) d u \\
\text { where } i=1,2, \ldots, M
\end{gathered}
$$

and

$$
\begin{gathered}
q_{n i}(t)=\int_{\substack{-\infty \\
\text { where }}}^{\infty} f\left(t-t_{n}-u\right) h_{n i}(u) d u \\
\text { and } i=1, \ldots, N
\end{gathered}
$$

Assuming an equalizer sampling phase of $t_{0}$, the sum of forward filter outputs, sampled at $t=t_{0}+b T$, is

$$
\begin{gathered}
x_{b}=v(b T)=\sum_{i=1}^{M} \sum_{l=-L 1}^{L 2} w_{i l}^{*} l \sum_{k=-\infty}^{\infty} s_{k 0} q_{n_{i}}\left(b T-k T-\left(l \tau_{s}-t_{0}\right)\right) \\
+\sum_{n=1}^{N} \sqrt{\frac{I_{n}}{S}} \sum_{\substack{\left.k=-\infty \\
+\nu_{i}\left(b T-\left(l \tau_{s}-t_{0}\right)\right]\right)}}^{\infty} s_{k n} q_{n i}\left(b T-k T-\left(l \tau_{s}-t_{0}\right)\right)
\end{gathered}
$$

From equations 3.4 and 3.13 , the second moment of the white noise components of $\mathrm{x}_{\mathrm{b}}, \nu_{i}\left(b T-\left(l \tau_{s}-t_{0}\right)\right)$, can be written as

$$
\begin{gathered}
\mathrm{E}\left[\nu_{i}(b T-k \tau) \nu_{j}^{*}(b T-l \tau)\right]=N_{0} f\left((k-l) * \tau_{s}\right) \delta_{1 j} \\
\text { for } \quad i, j=1, \ldots, M ; \quad k, l=1, \ldots, L .
\end{gathered}
$$

30 This parameter will hosvever be shown to have a marginal influence on performance in view of the time-varying nature of the channel. 
i.e. the white noise is uncorrelated (even in the short term) from antenna to antenna.

The analysis to follow is readily amenable to vector and matrix notation. Using bold face characters to represent vectors and matrices, one may define the $M^{*} L$ element forward filter tap weight vector $\mathbf{W}$ as

$$
\mathbf{W}=\left[w_{11}, \ldots, w_{1 L}, w_{21}, \ldots, w_{2 L}, \ldots, w_{M 1}, \ldots w_{M L}\right]^{T}
$$

where $T$ denotes transpose. In addition, the $M^{*} L$-element sampled continuous filter response vectors are:

$$
\begin{gathered}
\mathrm{q}_{k n}=\left[q_{n 1}\left(b T-k T+L_{1} \tau_{s}-t_{0}\right), \ldots, q_{n 1}\left(b T-k T-L_{2} \tau_{s}-t_{0}\right), \ldots\right. \\
\left.q_{n M}\left(b T-k T+L_{1} \tau_{s}-t_{0}\right), \ldots, q_{n M}\left(b T-k T-L_{2} \tau_{s}-t_{0}\right)\right]^{T} \\
\text { where } k=-\infty, \ldots, \infty \text { and } n=0,1, \ldots, N
\end{gathered}
$$

and the $M^{*} L$-element thermal noise vector is:

$$
\begin{aligned}
\mathbf{V}= & {\left[\nu_{1}\left(b T+L_{1} \tau_{s}-t_{0}\right), \ldots, \nu_{1}\left(b T-L_{2} \tau_{s}-t_{0}\right),\right.} \\
& \left.\ldots, \nu_{M}\left(b T+L_{1} \tau_{s}-t_{0}\right), \ldots, \nu_{M}\left(b T-L_{2} \tau_{s}-t_{0}\right)\right]^{T}
\end{aligned}
$$

The sampled sum of forward filter outputs $x_{b}$ can thus be expressed in vector notation as:

$$
x_{b}=\mathbf{W}^{* \mathbf{T}}\left(\sum_{k=-\infty}^{\infty} s_{k 00} \mathbf{q}_{k \mathbf{0}}+\sum_{n=1}^{N} \sqrt{\frac{I_{n}}{S}} \sum_{k=-\infty}^{\infty} s_{k n} \mathbf{q}_{k n}+\mathrm{V}\right)
$$

where ${ }^{*} T$ denotes conjugate transpose. The thermal noise energy affecting the decision variable 2 in Figure 3.1 is:

$$
\mathrm{E}\left[\left|\mathbf{W}^{* T} \mathrm{~V}\right|^{2}\right]=N_{0} \quad \mathbf{W}^{* \mathbf{T}} \mathbf{C}_{0} \mathbf{W}
$$

where

$$
\mathbf{C}_{0}=\left(\frac{1}{N_{0}}\right) E\left[\mathbf{V}^{* \mathbf{T}} \mathbf{V}\right]
$$

is the $\left(\left(M^{*} L\right)^{*}\left(M^{*} L\right)\right.$ ) thermal noise identity covariance matrix whose elements are defined by equation 3.17. Assuming [50] that the backward filter cancels the postcursor 
interference contribution from the desired portable and setting the decision symbol as $s 00$, the decision variable $z$ (see Figure 3.1 ) becomes

$$
z=\mathbf{W}^{*} \mathrm{~T}\left(\sum_{k=0}^{K} s_{k 0} \mathbf{q}_{k 0}+\sum_{n=1}^{N} \sqrt{\frac{I_{n}}{S}} \sum_{k=-h}^{K} s_{k n} \mathbf{q}_{k n}+\mathrm{V}\right)
$$

where the sum over $k$ has been truncated to $K$ terms [50] in view of the finite duration of the pulse function $q(t)$. In matrix form, the decision variable is now

$$
z=W^{* T}\left(s_{00 q_{00}}+V_{x}+V\right)
$$

where the interference (i.e. excluding AWGN) vector is

$$
\mathbf{V}_{\mathbf{x}}=\sum_{k=1}^{K} s_{k 0} q_{k 0}+\sum_{n=1}^{N} \sqrt{\frac{I_{n}}{S}} \sum_{k=-h^{-}}^{K} s_{k n} q_{k n}
$$

and since the thermal noise contribution from $\nu$ is independent of the ISI and cochannel interference in $V_{x}$, the effective (noise+interference) energy for a decision on $s_{00}$ is

$$
\begin{gathered}
\mathrm{E}\left[\left|\mathbf{W}^{* \mathbf{T}}\left(\mathbf{V}_{\mathbf{x}}+\mathbf{V}\right)\right|^{2}\right] \\
=N_{0} \mathbf{W}^{* \mathbf{T}} \mathbf{C}_{\mathbf{0}} \mathbf{W}+\mathbf{W}^{* \mathbf{T}_{\mathrm{E}}}\left[\mathbf{V}_{\mathbf{x}}^{* \mathbf{T}} \mathbf{V}_{\mathbf{x}}\right] \mathbf{W}
\end{gathered}
$$

Assuming that the symbol sequences in the $(\mathrm{N}+1)$ portables are all uncorrelated, i.e.

$$
\mathrm{E}\left[s_{k n} s_{l m}^{*}\right]= \begin{cases}\mathrm{E}\left[\left|s_{k n}\right|^{2}\right]=E_{s} & \text { for } k=l \text { and } n=m . \\ 0 & \text { for } k l \text { or } n m .\end{cases}
$$

the expectation in the second term of equation 3.27 becomes

$$
\begin{array}{r}
\mathrm{E}\left[\mathbf{V}_{\mathrm{x}}^{* \mathbf{T}} \mathbf{V}_{\mathbf{x}}\right]=E_{s} \sum_{k=1}^{K} \mathbf{q}_{k 0} \mathbf{q}_{k \mathbf{0}}^{* \mathbf{T}} \\
+\sum_{i=1}^{N} E_{s} \frac{I_{n}}{S} \sum_{k=-K}^{K} \mathbf{q}_{k n} \mathbf{q}_{k \mathbf{n}}^{*} \mathbf{T}
\end{array}
$$

The "interference" (i.e. ISI and interfering portables ) covariance matrix can now be defined as

$$
C_{i}=\frac{E\left[V_{x}^{* T} V_{x}\right]}{E_{s}}=\sum_{k=1}^{K} q_{k 0} q_{k 0}^{* T}+\sum_{n=1}^{N} \frac{I_{n}}{S} \sum_{k=-K}^{K} q_{k u} q_{k n}^{* T}
$$


The (noise and interference) energy is then

$$
E\left[\left|W^{* T}\left(V_{X}+V\right)\right|^{2}\right]=N_{0} W^{*} \mathbf{T}_{\mathbf{C}_{0}} \mathbf{W}+E_{s} \mathbf{W}^{* T} \mathbf{C}_{\mathbf{i}} \mathbf{W}
$$

The instantaneous, i.e. based on local means, signal-to-interference-plus-noise ratio (SINR) affecting the symbol decision at the "combiner" output $z$ is therefore

$$
\begin{aligned}
& \rho=\frac{E_{s}\left|W^{*} T_{q_{00}}\right|^{2}}{N_{0} W^{*} \mathbf{T}_{0} \mathrm{~W}+E_{s} W^{* T} C_{\mathrm{i}} \mathbf{W}}
\end{aligned}
$$

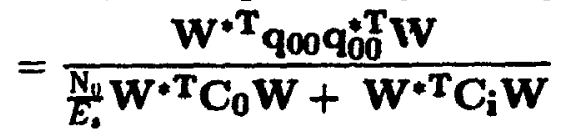

Since $C_{0}$ and $C_{i}$ are both Hermitian matrices, we may write

$$
\frac{N_{0}}{E_{s}} \mathbf{W}^{*} \mathbf{T} \mathbf{C}_{0} \mathbf{W}+\mathbf{W}^{*} \mathbf{T} \mathbf{C}_{\mathbf{i}} \mathbf{W}=\mathbf{W}^{*} \mathbf{T}\left(\frac{N_{0}}{E_{s}} \mathbf{C}_{0}+\mathbf{C}_{\mathbf{i}}\right) \mathbf{W}
$$

and thus define the interference-plus-noise (short-term average) covariance matrix $\mathbf{C}$ as

$$
\mathrm{C}=\frac{N_{0}}{E_{s}} \mathrm{C}_{0}+\mathbf{C}_{\mathbf{i}}
$$

The SINR now becomes

$$
\rho=\frac{W^{* T} q_{00} q_{00}^{* T} W}{W^{* T} \mathbf{C W}}=\frac{\left|W^{* T} q_{00}\right|^{2}}{W^{* T} \mathbf{C W}}=\frac{\left|W^{* T} \mathbf{C C}^{-1} q_{00}\right|^{2}}{W^{* T} \mathbf{C W}}
$$

This expression is to be maximized with respect to the forward filter weight vector W. Using a generalization of Schwartz's inequality on the last form of the preceding equation, it can then be shown [51,50] that the optimum set of forward filter taps is

$$
\mathbf{W}_{\text {opt }}=\mathbf{C}^{-1} \mathbf{q o o}_{00}
$$

thus yielding the optimized SINR

$$
\begin{aligned}
\rho_{\text {opt }} & =\frac{q_{00}^{*} \mathbf{C}^{-1} q_{00} q_{00}^{*} \mathbf{T}\left(C^{-1}\right)^{* T} q_{00}}{q_{00}^{* T}\left(C^{-1}\right)^{* T} \mathbf{T} C^{-1} q_{00}} \\
& =q_{00}^{* T} C^{-1} q_{00}
\end{aligned}
$$

Recall from Chapter 2 that one could choose some other optimality criterion (e.g. MMSE) than maximizing the SINR and obtain a result for $W_{\text {opt }}$ differing only by a scalar factor, thus yielding the same optimum SINR $\rho_{\text {opt }}$. One may then conclude that the set of forward filter weights (equation 3.36) maximizing the SINR also minimize the mean square error at the decision point $z$ in Figure 3.1, thus justifying the semantic equivalence of optimum baseband combining and minimum mean square error (MMSE) equalization of interference. 


\subsection{SINR distribution and bit error rate performance}

The SINR $q_{00}^{*} \mathbf{T} \mathbf{C}^{-1} \mathrm{q}_{00}$ in equation 3.37 is a Hermitian quadratic form in the complex gaussian variates of the random vector $q_{00}$ which is a function of the longterm fading channel statistics, i.e. equation $\rho_{o p t}$ is the optimum SINR for a particular channel realization. One must therefore average over the fading channel ensemble in order to determine average error or outage rates. A well-established technique [192] exists to determine the density function of such a quadratic form, via its characteristic function, provided that the matrix of the quadratic form $\mathbf{C}^{-1}$, is deterministic. However, this is only the case when there is no cochannel interference $(N=0)$ and no intersymbol interference (flat fading); the interference-plus-noise covariance matrix is then simply equal to the identity matrix $C_{0}$ as given by equation 3.23. In all other situations, a method of averaging over the quadratic form is not apparent because both the interference covariance matrix $\mathbf{C}$ and the vector $\mathbf{q}_{00}$ are functions of the long-term fading channel statistics. To circumvent this problem, Monsen has proposed [50] that the following two approximations be made:

1. Assume that the interfering symbols $s_{k n}$ are zero-mean gaussian distributed with mean-square value $\gamma^{2}$ as opposed to a (complex for four-level signaling) binary sequence. In this sense, the value of $\gamma^{2}$ should be twice as large for QPSK than for BPSK. This constant may be selected empirically or from theoretical considerations but should reflect the fact that a gaussian distribution exhibits a larger tail than a source sequence distribution. Hence, all disturbances (AWGN and interference from other portables) are now considered gaussian.

2. Approximate the random interterence (short-term average) covariance matrix components by their (long-term) means over the channel statistics, thus representing the interference as a non-fading disturbance and hence making $\mathbf{C}$ deterministic.

This approach is meaningful inasmuch as the error rate is affected more by signal fading than by intersymbol and cochannel interference. This is valid when the receiver is able to cancel cochannel interference and ISI created by filters and multipath delay 
spread to a level small compared with the decision thresholds. In this case, fading of the desired signal is the major impairment. On the other hand, when the cochannel interference level and the channel time dispersion are severe, these effects dominate and the non-fading gaussian noise representation for the ISI and the cochannel interference is not very realistic. However, such situations are of lesser interest, as they generally lead to poor error rate or outage performance.

With these assumptions, the interference covariance matrix becomes

$$
C_{i}=\gamma^{2} \sum_{k=1}^{K} \overline{q_{k 0} q_{k 0}^{* T}}+\sum_{n=1}^{N} \frac{I_{n}}{S} \gamma^{2} \sum_{k=-K}^{K} \overline{q_{k n} q_{k n}^{* T}}
$$

where, using equations $3.3,3.14,3.15$ and 3.19 , the elements of the $\left(\left(M^{*} L\right)^{*}\left(M^{*} L\right)\right)$ matrices $\overline{\mathbf{q k n}_{\mathbf{k}} \mathbf{q}_{\mathbf{k n}}^{* \mathbf{T}}}$ are given by

$$
\begin{aligned}
& \overline{\mathrm{q}_{\mathrm{kn}} \mathrm{q}_{\mathrm{kn}}^{\mathbf{T}}}\left((i-1) L+l+L_{1}+1,(j-1) L+r+L_{1}+1\right)= \\
& \int_{-\infty}^{\infty} f\left(t_{0}-t_{n}-k T-l \tau_{s}-u\right) f\left(t_{0}-t_{n}-k T-r \tau_{s}-u\right) \phi(u) d u \\
& \text { where } l=-L_{1}, \ldots, L_{2} ; \quad r=-L_{1}, \ldots, L_{2} \\
& \text { and } i=1,2, \ldots, M ; j=1,2, \ldots, M .
\end{aligned}
$$

The preceding reflects the fact that the signals received at the $M^{*} L$ diversity branches ${ }^{31}$ exhibit short-term correlation ( $C_{1}$ is a short-term covariance matrix); i.e. over the short-term interval where the channel can be considered as static, the multiple paths from a given signal pulse are correlated across the $M$ sets of $L$ diversity branches ${ }^{32}$. In the long term however, as specified by equation 3.3, the fading process is independent between the $M$ antennas and the multipath components at the $L$ forward filter taps are uncorrelated and thus independent as they are gaussian (GWSSUS model discussed in Chapter 2). Hence, whereas the effects of fading are alleviated by exploiting its

31 With an explicit space diversity order of $M$ and an implicit diversity order of $L$, the effective diversity order is $M^{*} L$.

32 An equalizer exploits this correlation across the $L$ taps in order to cancel interference, i.e. it cannot cancel a disturbance which is also white in the short-term (e.g. AWGN). 
long-term independence across the diversity branches, interference is suppressed by exploiting its short-term correlation across these same diversity branches.

The matrix $\mathbf{C}^{-1}$ is now a deterministic function of the pulse shaping characteristics and the channel statistics. Hence, one may now use Turin's method [192] to determine the probability density function of $\rho_{\text {opt }}$. In addition, as equation 3.39 makes $\mathrm{C}^{-1}$ Hermitian positive semi-definite, the characteristic function of $\rho_{o p t}$ is defined as a Laplace (as opposed to Fourier) transform of the probability density function of the quadratic form in equation 3.37. This yields [192, 193]

$$
G_{\rho_{o p t}}(s)=\int_{0}^{\infty} \exp \left(-s \rho_{o p t}\right) p\left(\rho_{o p t}\right) d \rho_{o p t}=\frac{1}{\left.\operatorname{det}_{1} \mathrm{I}+2 s \mathbf{R C ^ { - 1 }}\right)}
$$

where $I$ is the $\left(\left(M^{*} L\right)^{*}\left(M^{*} L\right)\right)$ identity matrix and

$$
\mathbf{R}=\frac{1}{2} \overline{\left(\mathbf{q}_{00}-\overline{\mathbf{q}_{00}}\right)\left(\mathbf{q}_{00}-\overline{\mathbf{q}_{00}}\right)^{* T}}
$$

is the covariance matrix of the complex gaussian variates of the quadratic form. Assuming Rayleigh fading, all the elements of $\overline{q 00}$ are equal to zero and

$$
G_{\rho_{o p t}(s)}=\frac{1}{\operatorname{det}\left(\mathbf{I}+s \widetilde{q_{00} \mathbf{q}_{00}^{* T}} \mathbf{C}^{-1}\right)}
$$

From equations 3.3 and 3.14, $\overline{\mathbf{q}_{00} \mathrm{q}_{00}^{* \mathrm{~T}}}$ is Hermitian (as well as block-diagonal). As $\mathrm{C}^{-1}$ is also Hermitian, and using the fact that the determinant of any Hermitian matrix is equal to the product of its eigenvalues,

$$
G_{\rho_{\text {opt }}(s)}=\prod_{t=1}^{M L}\left(1+s \lambda_{t}\right)^{-1}
$$

where the $\left\{\lambda_{i}\right\}$ are the eigenvalues of the $\left(\left(M^{*} L\right)^{*}\left(M^{*} L\right)\right)$ Hermitian matrix $\overline{\mathbf{q}_{00} \mathbf{q}_{00}^{*} \mathbf{T}} \mathbf{C}^{-1}$.

A closed-form expression for the probability density function (for a particular set of system parameters) can thus be obtained by inverting the partial fraction expansion of its Laplace transform, namely

$$
G_{\rho_{o p t}}(s)=\sum_{i=1}^{\Lambda} \sum_{j=1}^{n_{1}} \frac{A_{i j}}{\left(1+s \lambda_{\imath}\right)^{3}}
$$


where $\Lambda$ is the number of distinct eigenvalues $\lambda_{\mathfrak{i}}$ with multiplicity $n_{\mathfrak{i}}$. The partial fraction expansion coefficients $A_{i j}$ can be found recursively as shown in Appendix $A$.

As the effect of ISI and $\mathrm{CCI}$ has now been replaced by non-fading gaussian noise components in order to make the covariance matrix $\mathbf{C}$ deterministic, the average bit error rate for a particular modulation scheme can be found by taking the average of the error rate expression (for white gaussian noise) over the fading channel ensemble [50]. For BPSK, the SINR per symbol $\rho_{\text {opt }}$ is equal to the SINR per bit; hence the BER expression for coherently detected BPSK [71] is simply $\frac{1}{2} \operatorname{erfc}\left(\sqrt{\rho_{\text {opt }}}\right)$. The average error rate over Rayleigh fading is thus

$$
\overline{P_{b-b p s k}}=\int_{0}^{\infty} \frac{1}{2} \operatorname{erfc}\left(\sqrt{\rho_{o p t}}\right) p\left(\rho_{\text {opt }}\right) d \rho_{o p t}
$$

Expressing $\overline{P_{b-\text { dpsk }}}$ in terms of the eigenvalues $\lambda_{i}$ and the partial fraction expansion coefficients $A_{i j}$ is no trivial matter. It can be done by rearranging a simplifying formula [128] for an integral of the form $\int_{0}^{\infty} \exp (-x t) \operatorname{erf}(\sqrt{y t}) d t$. However, if one upper bounds the complementary error function by an exponential function ${ }^{33}$, i.e. $\operatorname{erf} c\left(\sqrt{\rho_{\text {opt }}}\right) \leq e^{-\rho_{\text {opt }}}$, the resulting expression for the average BER becomes

$$
\overline{P_{b-b p s k}} \leq \int_{0}^{\infty} \frac{1}{2} \exp \left(-\rho_{o p t}\right) p\left(\rho_{o p t}\right) d \rho_{o p t}
$$

which, from equations 3.3 .2 .40 and 3.3.2.41, is simply equal to

$$
\overline{P_{b-b p s k}} \leq \frac{1}{2} G_{\rho_{o p t}}(1)=\frac{1}{2 \operatorname{det}\left(\mathrm{I}+\overline{\mathbf{q}_{00} \mathbf{q}_{00}^{* T}} \mathbf{C}^{-1}\right)}
$$

Although it simplifies the anal; :s, the exponential bound will lead to pessimistic results with respect to the coherent detection case. However, if differential encoding is used in order to eliminate a phase ambiguity (of $180^{\circ}$ for BPSK and $90^{\circ}$ for CiSK)

33 It is shown in [194, p.83] that the $Q$ function $Q(\alpha)=\frac{1}{\sqrt{2 \pi}} \int_{\alpha}^{\infty} e^{-\frac{\alpha^{2}}{3}} d \alpha$ can be upper bounded by $Q(\alpha) \leq \frac{1}{2} e^{-\frac{\alpha^{2}}{2}}$ where the bound becomes tighter as $\alpha$ increases. In terms of the complementary

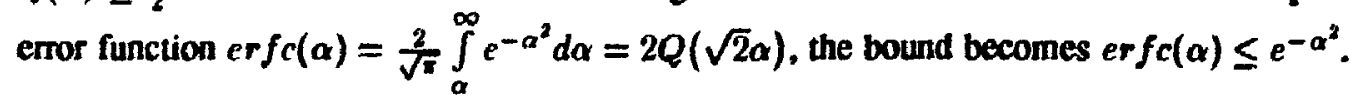


between transmitter and receiver ${ }^{34}$ and when practical degradation (e.g. imperfect carrier recovery) is taken into account, equation 3.47 should yield a reasonable estimate of realizable performance.

For QPSK, tie symbol error probability in gaussian noise is given by [71] (p.169)

$$
\begin{aligned}
P_{s-q p s k} & =\operatorname{erfc}\left(\sqrt{\frac{\rho_{o p t}}{2}}\right)\left[1-\frac{1}{4} \operatorname{erfc}\left(\sqrt{\frac{\rho_{\text {opt }}}{2}}\right)\right] \\
& \approx \operatorname{erfc}\left(\frac{\sqrt{2 \rho_{\text {opt }}}}{2}\right)
\end{aligned}
$$

where the latter approximation improves as the SINR per symbol, $\rho_{o p t}$ increases. If. as in the BPSK case, the complementary error function is bounded by an exponential function i.e. $\operatorname{erfc}\left(\frac{\sqrt{2 \rho_{\mathrm{opt}}}}{2}\right) \leq e^{-\frac{\rho_{\text {opp }}}{2}}$, the QPSK symbol error probability averaged over Rayleigh fading becomes

$$
\overline{P_{s-q p s k}} \leq \int_{0}^{\infty} \exp \left(-\frac{\rho_{o p t}}{2}\right) p\left(\rho_{o p t}\right) d \rho_{o p t}
$$

Finally, if a Gray code [71] is used to map the 2-bit symbols into signal phases, adjacent signal phases will differ only in a single bit. Since the majority of symb.jl ent $r s$ result in the erroneous selection of an adjacent phase to the true phase, most syniool errors will contain a single bit error and the average QPSK bit error probability can then be written as

$$
\begin{aligned}
\overline{P_{b-q p s k}} \leq & \frac{1}{2} \int_{0}^{\infty} \exp \left(-\frac{\rho_{o p t}}{2}\right) p\left(\rho_{o p t}\right) d \rho_{o p t}= \\
& \frac{1}{2} G_{\rho_{o p t}}(0.5)=\frac{1}{2 \operatorname{det}\left(\mathbf{I}+0.5 \overline{\mathbf{q}_{00 \mathbf{q}_{00}^{* \mathbf{T}}}} \mathbf{C}^{-1}\right)}
\end{aligned}
$$

Considering the limiting case of flat (Rayleigh) fading and no cochannel interference $(N=0)$ and no diversity $(M=L=1)$, equations 3.47 and 3.50 yield the same BER expression for QPSK and BPSK i.e.

$$
\begin{cases}\overline{P_{b-b p s k}} \leq \frac{1}{2\left(1+\frac{E_{a}}{N_{1}}\right)}=\frac{1}{2\left(1+\frac{E_{b}}{N_{10}}\right)} & \text { where } E_{s}=E_{b} \\ \overline{P_{b-q p s k}} \leq \frac{1}{2\left(1+\frac{19 E_{2}}{N_{i 1}}\right)}=\frac{1}{2\left(1+\frac{E_{b}}{N_{10}}\right)} & \text { where } E_{s}=2 E_{b}\end{cases}
$$

34 Differential encoding of the transmitted data tends to degrade performance slightly [50]. 


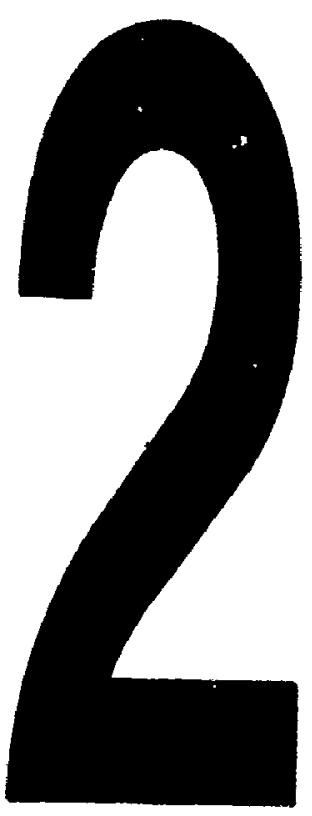

PM-1 31/2" $x 4$ " PHOTOGRAPHIC MICROCOPY TARGET NBS 1010a ANSI/ISO $\# 2$ EQUIVALENT

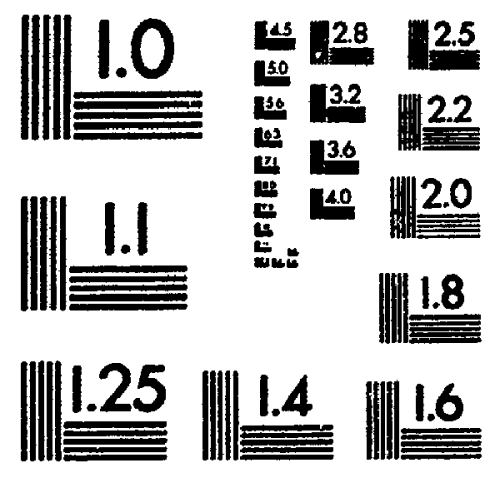


Equation 3.51 actually corresr onds to the binary DPSK BER expression in flat Rayleigh fading and gaussian noise [71, p.468]. Hence, in flat Rayleigh fading with no CCI, the BER performances of BPSK and QPSK, which as shown in Appendix B are identical, are approximated by the binary DPSK BER expression. It is by no means suggested however, to implement differential detection in a receiver with equalization as this could be difficult to realize. The exponential bound is simply used as a means of simplifying the analysis and of accounting for the degradations resulting from differential encoding or imperfections in carrier recovery. Finally, in the presence of cochannel or intersymbol interference, the BER performance of BPSK and QPSK will differ as for the latter, not only the desired symbol but also the interfering symbols have increased energy (recall equation 3.32). In this way, the model accounts for the effect of cross-rail interference which is absent in BPSK systems [195].

\subsection{Bit error rate bound for coded system}

The bit error rate expressions of equations 3.47 and 3.50 assume an uncoded system. Channel coding provides an additional form of diversity $[163,112,80]$ to combat the detrimental effects o a time-varying dispersive channel. It is well known $[71,57]$ that a simple upper bound on the error probability for soft-decision Viterbi decoding of a rate $\frac{1}{n}$ (i.e. bandwidth-expanding) convolutional code can be expressed as

$$
\overline{P_{b}} \leq \sum_{d=d_{\text {free }}}^{\infty} \beta_{d} \overline{P_{2}(d)}
$$

where $\overline{P_{2}(d)}$ is the pairwise error probability (averaged over the fading distribution) for sequences that differ in $d$ bits, $\beta_{\mathrm{d}}$ is obtained from the coefficients of the first derivative of the code's transfer function and $d_{\text {free }}$ is the minimum free distance of the convolutional code. On a time-varying (Rayleigh) fading channel, the potential diversity improvement of coding is best exploited by transmitting the signal components carrying the same information (i.e. the bits or symbols of a given codeword) in multiple time intervals mutually separated by an amount exceeding the coherence time of the channel. In other words, the codewords should be interleaved prior to modulation 
to breakup error bursts to which the Viterbi decoder is very sensitive. However, the indoor channel is a very slowly-varying or quasi-static medium. Thus, as discussed in Chapter 2, the interleaving depth required to "cover" an average fade duration would be unreasonably large, leading to huge memory requirements and causing excessive delay. In practice, interleaving cannot therefore eliminate the effect of $;$;rror bursts (i.e. randomize errors) completely. The receiver structure shown in Figure 3.1 does not use interleaving but, by exploiting the implicit diversity of the dispersive channel, provides a large effective diversity order $M^{*} L$ to maximize the SINR at the Viterbi decoder input. This will hopefully yield infrequent error bursts and thus small outage probabilities at error rates of interest. However, to make the analysis tractable, the signal waveforms corresponding to various coded bits will be assumed to undergo independent fading (as if interleaving of nearly infinite depth were used); the resulting curves will therefore represent a lower bound (in terms of BER) on realizable performance of the coded system. The bit-by-bit simulation of Chapter 4 and the semi-analytical technique with Monte Carlo averaging of Chapter 5 will later be used to assess the effect of error bursts when no interleaving is used. The instantaneous SINR per sequence of $d$ transmitted (coded) bits ${ }^{35}$

$$
\rho_{d}=\sum_{i=1}^{d} \rho_{c_{i}}
$$

is thus equal to the sum of $d$ statisticaily independent identically distributed random variables $\rho_{c_{i}} \quad(i=1, \ldots d)$ representing the instantaneous SINR per coded bit.

The Viterbi soft-decision decoder computes path metrics of the form [71, 57]

$$
U^{(r)}=\sum_{j=1}^{B} \sum_{m=1}^{n} z_{j m}\left(2 c_{j m}^{(r)}-1\right)
$$

where $r$ denotes any one of the competing paths at each node, $B$ is the number of branches in a path, $n$ is the number of bits per codeword, the $\left\{c_{j m}\right\}$ are the (transmitted) coded bits on the $j^{\text {th }}$ branch of the code and the $\left\{z_{j m}\right\}$ are the unquantized inputs to the decoder (refer to Figure 3.1). Since the code is linear, one may determine the 35 Only binary codes are considered herein. 
conditional (i.e. fixed SINR or time-invariant) pairwise error probability by comparing the all-zero path $(r=0)$ and another $(r=1)$ with $d$ ones, i.e.

$$
\begin{aligned}
P_{2}\left(d, \rho_{d}\right) & =\operatorname{Pr}\left(U^{(1)}-U^{(0)} \geq 0\right) \\
& =\operatorname{Pr}\left(\sum_{j=1}^{B} \sum_{m=1}^{n} z_{j m}\left(c_{j m}^{(1)}-c_{j m}^{(0)}\right) \geq 0\right)
\end{aligned}
$$

Since the coded bits in the two paths are identical except in the $d$ positions, this simplifies to

$$
P_{2}\left(d, \rho_{d}\right)=\operatorname{Pr}\left(\sum_{b=1}^{d} z_{b} \geq 0\right)
$$

where the index $b$ runs over the set of $d$ bits in which the paths differ and the set $\left\{z_{b}\right\}$ represents the input to the decoder for these $d$ bits.

The key to obtaining a coded BER bound lies in noticing that equation 3.56 also denotes the probability of error for $d$ time-invariant AWGN diversity channels differing only in attenuation and phase shift. Closed-form expressions can be obtained for various (binary) modulation schemes as special cases of the general quadratic form in complex gaussian random variables for multichannel signaling [71]. For the sake of analytical simplicity and consistency with the uncoded case, the exponential (i.e. differential detection) bound is once again used to approximate the practical performance of coherent BPSK and QPSK. Hence, for binary DPSK, this method yields after a lengthy derivation $^{36}$

$$
P_{2}\left(d, \rho_{t}\right)=\frac{1}{2^{2 d-1}} e^{-\rho_{d}} \sum_{k=0}^{d-1} b_{k} \rho_{d}^{k}
$$

where

$$
b_{k}=\frac{1}{k !} \sum_{n=0}^{d-1-k} \frac{(2 d-1) !}{(2 d-1-n) ! n !}
$$

In order to determine the probability density function (p.d.f.) of $\rho_{\mathrm{d}}$ (required to find the average pairwise error probability), one first obtains the p.d.f. of $\rho_{c}$ (equation 3.54) from the Laplace transform of this p.d.f., $G_{\rho_{c}}(s)$. The latter is determined in the

The reader is referred to [71, sec. 4.4 and appendix 4B] for the details. 
Same way as for the uncoded system (recall equation 3.42) with the exception of the interference-plus-noise covariance matrix (equation 3.34) which is now

$$
\mathrm{C}=\frac{N_{n}}{E_{s} R c} \mathrm{C}_{0}+\mathbf{C}_{\mathbf{i}}
$$

where $\boldsymbol{K}_{\boldsymbol{c}}$ denotes the code rate and where $E_{s}$ represents the energy per information symbol, i.e. per pair of information bits in QPSK. As for a given $E_{s}, E_{b_{B P S K}}=$ $2 E_{b_{Q P S K}}$, this procedure yields ${ }^{37}$

$$
G_{\rho_{c}}(s)= \begin{cases}\frac{1}{\operatorname{det}\left(\mathrm{I}+s \mathrm{q}_{\left.00 \mathrm{q}_{00}^{\circ \mathcal{T}} \mathrm{C}^{-1}\right)}\right.} & \text { for } B P S K \\ \frac{1}{\operatorname{det}\left(\mathrm{I}+0.5 s \mathrm{q}_{00} \mathrm{q}_{00}^{\circ \bar{T}} \mathrm{C}^{-1}\right)} & \text { for } Q P S K\end{cases}
$$

As $\rho_{\mathrm{d}}$ is assumed to be the sum of $d$ statistically independent and identically distributed random variables $\rho_{c_{t}}$, the characteristic function of $\rho_{d}$ is simply the characteristic function of the $\rho_{c}$ raised to the $d^{\text {h }}$ power. As $p_{P_{c}}\left(\rho_{c}\right)=0$ for $\rho<0$, the characteristic function is defined as a Laplace transform instead of a Fourier transform (with a negative sign in the exponential), yielding the p.d.f. of $\rho_{\mathrm{d}}$ as

$$
p_{P_{d}}\left(\rho_{d}\right)=\mathrm{L}^{-1}\left[\left(\mathrm{G}_{\rho_{\mathrm{c}}}(\mathrm{s})\right)^{\mathrm{d}}\right]
$$

where $\left.L^{-1} /.\right]$ denotes inverse Laplace transform.

From equation 3.57 , the average pairwise error probability $\overline{P_{2}(d)}$ is

$$
\overline{P_{2}(d)}=\int_{0}^{\infty} P_{2}\left(d, \rho_{d}\right) p_{P_{d}}\left(\rho_{d}\right) d \rho_{d}=\frac{1}{2^{2 d-1}} \sum_{k=0}^{d-1} b_{k} \int_{0}^{\infty} \rho_{d}^{k} e^{-\rho_{d}} p_{P_{d}}\left(\rho_{d}\right) d \rho_{d}
$$

where the $b_{k}$ are given by equation 3.58. Defining the Laplace transform of $p p_{d}\left(\rho_{d}\right)$ as $G_{\rho_{d}}(s)$, one may write (recall equation 3.40 )

$$
\frac{d^{k}}{d s^{k}} G_{\rho_{d}}(s)=(-1)^{k} \int_{0}^{\infty}\left(\rho_{d}\right)^{k} e^{-s \rho_{d}} p_{\mathrm{P}_{d}}\left(\rho_{d}\right) d \rho_{d}
$$

37 The distinction between the BPSK and QPSK cases is obtained as follows. Let $G_{X}(s)=$ $\int_{0}^{\infty} \exp (-s x) p_{X}(x) d x$ and $G_{Y}(s)=\int_{0}^{\infty} \exp (-s y) p_{Y}(y) d y$ be the Laplace transforms of the p.d.f.'s of the random variables $X$ and $Y$ where $Y=2 X$, i.e. $p_{Y}(y)=\frac{1}{2} p_{X}(x)$. Expressed in terms of $G_{Y}(s), G_{X}(s)$ is then simply $G_{X}(s)=\int_{0}^{\infty} \exp \left(-\frac{s y}{2}\right) 2 p_{Y}(y) \frac{d y}{2}=G_{Y}\left(\frac{\rho}{2}\right)$. 
which, upon substitution in equation 3.57 , yieids

$$
\begin{aligned}
\overline{P_{2}(d)} & =\frac{1}{2^{2 d-1}} \sum_{k=0}^{d-1} b_{k}(-1)^{k}\left(\left.\frac{d^{k}}{d s^{k}} G_{\rho_{d}}(s)\right|_{s=1}\right) \\
& =\frac{1}{2^{2 d-1}} \sum_{k=0}^{d-1} b_{k}(-1)^{k}\left(\left.\frac{d^{k}}{d s^{k}}\left(G_{\rho_{c}}(s)\right)^{d}\right|_{s=1}\right)
\end{aligned}
$$

The pairwise error probability $P_{2}(d)$ can be expressed in terms of the eigenvalues $\lambda_{i}$ of the marrix $\overline{\mathbf{q 0 0 q}_{00}^{* T}} \mathbf{C}^{-1}$ for BPSK or of the matrix $0.5 \overline{\mathbf{q 0 0}_{00}^{* T}} \mathbf{C}^{-1}$ for QPSK via the partial fraction expansion of $\left(G_{\rho_{c}}(s \grave{j})^{d}\right.$, i.e. (refer to equation 3.44)

$$
\left(G_{p_{c}}(s)\right)^{d}=\sum_{i=1}^{\Lambda} \sum_{j=1}^{n_{i} d} \frac{B_{i j}}{\left(1+s \lambda_{i}\right)^{j}}
$$

where the coefficients $B_{i j}$ can be obtained using the recursive procedure outlined in Appendix A. After some simple manipulations, it is quickly apparent that

$$
\frac{d^{k}}{d s^{k}}\left(\left(G_{\rho_{c}}(s)\right)^{d}\right)=(-1)^{k} \sum_{i=1}^{\Lambda} \sum_{j=1}^{n_{1} d} \frac{B_{i j}\left(\lambda_{i}\right)^{k}(j+k-1) !}{(j-1) !\left(1+s \lambda_{i}\right)^{j+k}}
$$

Finally, the average pairwise error probability becomes

$$
\overline{P_{2}(d)}=\frac{1}{2^{2 d-1}} \sum_{k=0}^{d-1} b_{k} \sum_{i=1}^{\Lambda} \sum_{j=1}^{n_{1} d} \frac{B_{i j}\left(\lambda_{i}\right)^{k}(j+k-1) !}{(j-1) !\left(1+\lambda_{i}\right)^{j+k}}
$$

and the bit error probability (averaged over the fading SINR distribution) is obtained from equation 3.52 .

Note that, as in the uncoded system, the BER performance in flat fading with no cochannel interference (and $M=L=1$ ) is identical for BPSK and QPSK; for this limiting case (with coding), there is one eigenvalue $\lambda=\left(\frac{E_{a}}{N_{11}}\right) R c=\left(\frac{E_{6}}{N_{11}}\right) R c$ for BPSK and $\lambda=0.5\left(\frac{E_{s}}{N_{0}}\right) R c=\left(\frac{E_{b}}{N_{0}}\right) R c$ for QPSK. The function $\left(G\left(\rho_{c}(s)\right)\right)^{d}$ has then one eigenvalue of multiplicity $d$, i.e. $\Lambda=1, n_{i} d=I$, and $B_{i j}=B_{1 j}=0 \quad(j=1, \ldots,(d-1))$, whereas $B_{1 d}=1$. Equation 3.67 then collapses to

$$
\overline{P_{2}(d)}=\left(\frac{1}{\left(2^{2 d-1}\right)(d-1) !\left(1+\frac{E_{b} R c}{N_{11}}\right)^{d}}\right) \sum_{k=0}^{(d-1)} b_{k}\left(\frac{\left(\frac{E_{b} R c}{N_{0}}\right)}{1+\left(\frac{E_{k} R c}{W_{11}}\right)}\right)(d+k-1) !
$$


which, as seen from Appendix B, is found to match the expression obtained by Proakis [71, chapter 7] for binary DPSK with convolutional coding and soft-decision Viterbi decoding over a flat Rayleigh fading channel.

\subsection{Summary of analytical assumptions}

Prior to presenting and discussing the analytical results, it is worthwhile to recap the underlying assumptions in the analysis. These are namely

1. The intersymbol and cochannel interference symbols are assumed to be zero-mean gaussian distributed with mean-square value $\gamma^{2}$. All results shown herein assume $\gamma^{2}=0.5$ for BPSK and $\gamma^{2}=1.0$ for QPSK.

2. The short-term average random interference covariance matrix components (i.e. interference power) are approximated by their long-term means over the fading channel statistics. This yields a non-fading characterization of interference.

3. The realizable performance of coherently detected BPSK and QPSK with differential encoding is approximated by an exponential curve.

4. Feedback decisions are assumed to be correct.

5. For the coded system, errors at the Viterbi decoder input are assumed to be perfectly randomized. For the indoor channel, this implies interleaving of very large depth.

6. The Viterbi decoder path memory is assumed infinite.

\subsection{Results}

The effects of various system parameters, such as the number of receiver antennas $M$, number of interferers $N$, signal-to-interference ratio $S / I$, equalizer sampling phase $t_{0}$, number of forward filter taps $L, \mathrm{~ms}$ delay spread $\sigma_{\mathrm{d}}$ and thus data rate, etc., are now evaluated based on the analysis just described. BPSK and QPSK modulations are compared on the basis of equal information throughput and bandwidth efficiency. The relative merit of increasing the space diversity order and using convolutional coding with Viterbi decoding is also compared. Most results deal with a target symbol rate of $10 \mathrm{Mbits} / \mathrm{s}$, i.e. $\sigma_{d}=0.25 T$ for a typical mean ms delay spread of $25 \mathrm{~ns}$. In 
addition, a forward filter tap spacing $\tau_{\mathrm{s}}$ of $\mathrm{T} / 2$ is used and a common exponential delay profile $\phi(\tau)=\frac{1}{\sigma_{d}} e^{-\frac{\tau}{\sigma_{d}}}$ is assumed for all portable to base station antenna paths. When convolutional coding with soft-decision Viterbi decoding is employed, a rate $1 / 2, d_{\text {free }}=5$, constraint length 3 code with code generators 5 and 7 is selected. This a maximum free distance code, i.e. it has the largest possible $d_{\text {free }}$ for a given rate and a given constraint length [71]. This simple, short constraint length code yields only four trellis states thus minimizing coder and decoder complexity. The analytical results of Figures 3.3 to 3.17 and table 3.1 can be found in pages 83 to 98 .

\subsubsection{Principle of optimum combining}

Figure 3.3 shows the performance improvement obtained as the fraction of the total interference-plus-noise ${ }^{38}$ power consisting of interference increases. For a given SINR $\Gamma$ and a set of interferer-to-noise ratios $\frac{E_{S_{n}}}{N_{11}}(n=1, \ldots N)$, the values of $\frac{E_{t}}{N_{11}}$ and $\frac{l_{n}}{S}$ required for the computation are found from equation 3.10 i.e. $\frac{E_{k}}{N_{11}}=\Gamma\left(1+\sum_{n=1}^{N} \frac{E_{n n}}{N_{11}}\right)$ after which $\frac{I_{n}}{S}$ is found from $\frac{E_{s n}}{N_{10}}=\left(\frac{E_{v_{n}}}{E_{s}}\right)\left(\frac{E_{2}}{N_{11}}\right)=\left(\frac{I_{n}}{S}\right)\left(\frac{E_{2}}{N_{11}}\right)$. For an average bit error rate of $10^{-3}$ and with $\frac{E_{S_{n}}}{N_{1}}=1,(n=1, \ldots N)$, the performance improvement over the case of no cochannel interference for $M=2(M=3)$ antennas is then found to be $1.4 \mathrm{~dB}(2.2 \mathrm{~dB})$ in the case of $\mathrm{N}=1$ interferer and $2.5 \mathrm{~dB}(3.1 \mathrm{~dB})$ in the case of $\mathrm{N}=2$ interferers. Hence, for a given set of $\frac{E_{S n}}{N_{n}},(n=1, \ldots . N)$, the performance improves as $M$ increases because of explicit space diversity and also as $N$ increases since the optimum baseband combiner effectively cancels the interference by exploiting its short-term correlation across both the explicit anc implicit diversity branches. This characieristic of optimum baseband combining was also observed by Winters [53] for optinum pre-detection combining albeit only with explicit space diversity. As opposed to the latter case however, the $\mathrm{N}=0$ case in Figure 3.3 dces not represent $100 \%$ white noise as there is ISI resulting from the rms delay spread of $0.25 \mathrm{~T}$. In this sense, this $\mathrm{N}=0$

38 Recall from Chapter 2 that noise refers to the additive white gaussian noise and interference to the sum of intersymbol interference and cochannel interference from other portables. In the analysis, both impairments are modeled as having gaussian statistics; however, whereas the interference exhibits short-term correlation between the diversity branches, the noise is uncorrelated even in the short-term. 
case cannot be considered as maximal-ratio combining as interference cancellation is still being performed. Furthermore, performance will improve, albeit to a lesser degree, with increasing $N$ even in the case of $M=1$ antenna because of the capability of the equalizer to reject interference. In the same way, the improvement would be observed with $L=1$ forward filter tap just as with an optimum pre-detection combiner.

\subsubsection{Equalizer sampling phase}

The results of Figure 3.3 were generated with $L=3$ taps in each of the $M$ forward filters. This number was kept small in order to minimize complexity but, as shown in Figures 3.4, 3.5 and 3.6 whose results are summarized in table 3.1, the sensitivity to equalizer sampling phase can be significant with such a small number of taps. It should be noted that, when referring to pulse shapes, the term rectangular denotes the case of no shaping (i.e. very wide bandwidth) and should not be confused with a pulse having a rectangular spectrum such as a spectrally raised cosine pilse with $0 \%$ rolloff. As discussed in Chapter 2, sampling phase dependency increases with excess bandwidth. This time-rectangular case, although of strictly theoretical interest, should thus yield an upper bound on sampling phase sensitivity.

For a single explicit space diversity branch and no cochannel interference (i.e. the simple DFE case with only ISI), the $\frac{E_{b}}{N_{a}}$ required for a $10^{-2}$ average BER is seen to vary with sampling phase by as much as $1.40 \mathrm{~dB}$ for a 50\% rolloff SRC pulse and in the limiting case of the very wide bandwidth time-rectangular pulse by $2.04 \mathrm{~dB}$. With $M=2$ antennas, Figure 3.5 shows that the sampling phase sensitivity is now reduced, for the $50 \%$ SRC pulse, to $0.37 \mathrm{~dB}$ and only increases to $0.39 \mathrm{~dB}$ when a cochannel interferer is added $(\mathrm{N}=1)$. With the time-rectangular pulse however, the sensitivity goes down only slightly to $1.98 \mathrm{~dB}$ with $\mathrm{M}=2$ antennas and increases significantly to 3.06 $\mathrm{dB}$ when the cochannel interferer is added. With $\mathrm{M}=3$ antennas, Figure 3.6 then shows sensitivities of only $0.17 \mathrm{~dB}$ and $0.18 \mathrm{~dB}$ for the $50 \%$ SRC pulse with $\mathrm{N}=0$ and $\mathrm{N}=1$ respectively while for the time-rectangular pulse, the corresponding values are 1.96 $\mathrm{dB}$ and $2.17 \mathrm{~dB}$. For the $50 \% \mathrm{SRC}$ pulse, diversity thus substantially reduces (i.e. by nearly a fator of four from $M=1$ to $M=2$ and more than a factor of two from $M=2$ 
to $M=3$ ) the sampling phase sensitivity while minimizing the influence of a cochannel interferer. However for the wide bandwidth time-rectangular pulse, diversity only marginally reduces sampling phase sensitivity while a cochannel interferer increases sampling phase sensitivity by more than $50 \%$ for $M=2$ and by about $10 \%$ for $M=3$. The ability of explicit space diversity to reduce sampling phase sensitivity and to minimize its increase when cochannel interference is added thus varies inversely with the pulse bandwidth. As increasing the explicit space diversity order $M$ effectively adds more taps, sampling phase sensitivity is alleviated just as when taps are added to a single forward filter. However, the diversity effect provided by the different shortterm impulse responses to each of the $M$ forward filters also plays a role in minimizing sampling phase sensitivity.

The cyclostationary nature of cochannel interference tends to exacerbate this dependency on sampling phase. This effect, which has been recently studied for the subscriber loop channel $[196,197,198,199]$ results from the interference statistics being periodically stationary with a period of one symbol interval. As will be shown in Chapter 4, the cyclostationary nature of cochannel interference has been verified by simulation for a channel model with an exponential delay profile. This result is of importance as the effect of interference is only relevant at the sampling instants in the receiver. By choosing the appropriate sampling phase, a performance improvement over the stationary noise case can be achieved. Hence, if the sampling phase is judiciously selected, the gain obtained in going from $\mathrm{N}=0$ to $\mathrm{N}>0$ in Figure 3.3 will be larger in the presence of cyclostationary interference than in the presence of stationary interference. An improvement in terms of SINR is still observed when going from $\mathrm{N}=0$ to $\mathrm{N}=1$ in the presence of stationary interference provided it exhibits (short-term) correlation between antennas and/or between forward filter taps, i.e. when the interference covariance matrix $\mathbf{C}_{\mathbf{i}}$ (equation 3.30) has off-diagonal elernents. Estimating performance with such colored stationary noise would involve averaging the covariance matrix elements over the symbol interval $T$ as in $[196,197]$. With the more practical $50 \%$ SRC pulse shape and with a number of antennas $M$ of two or more, the influence of interferer cyclostationarity is however somewhat marginal as sampling phase sen- 
sitivity is then limited to $0.4 \mathrm{~dB}$. Note that this dependency on sampling phase couid also be reduced by using a number of fractionally-spaced forward filter taps larger than 3 and also in a situation where a large number of equal-power interferers with random timing phases are present, thus resulting in interference with nearly stationary statistics. However, as discussed in Chapter 2, indoor measurements have indicated that a single interferer usually dominates, and thus sampling phase remains an imponant parameter, particularly if there is a single explicit space diversity branch, if the number of forward filter taps is small and if the pulse bandwidth is large.

It is observed that, for the most sensitive case of the wide bandwidth timerectangular pulse, the best sampling phase for an exponential delay profile with an rms delay spread of $0.25 \mathrm{~T}$ is $0.375 \mathrm{~T}$. A large number of the following analytical results are thus generated with this sampling phase. This value is optimum in an average sense; the best sampling phase will actually vary with each set of channel realizations on the various portable to base station antenna paths. Hence, the cyclostationary nature of interference could be better exploited by continuously optimizing the sampling phase as opposed to using the best fixed sampling phase. This issue will be examined in Chapter 5.

It should also be noted that the influence of the (fixed) timing phase of a single interferer with respect to the desired signal was found to be far smaller than the effect of sampling phase and even negligible. Of course, this is strictly of theoretical interest as the timing phase of cochannel interference will typically vary randomly ${ }^{39}$ in a cellular radio system. As an example, for $M=2, N=1, \frac{S}{I}=1.0 d B, t_{0}=0.375 T$, $L=3, L 1=2, L 2=0$ and $\sigma_{d}=0.25 T$, the variation in $\frac{E_{b}}{N_{0}}$ required for a $10^{-3}$ average BER was virtually nil over eight different timing phases for both a 50\% SRC and a timerectangular pulse. At $\frac{E_{p}}{N_{0}}=30 d B$ where the AWGN power is very small relative to the cochannel interference, timing phase still produced no significant performance variation for the 50\% SRC pulse and a variation of no more than $0.5 \mathrm{~dB}$ for the time-rectangular pulse. The negligible effect of interferes timing phase can be attributed to the fact that the interferer and the desired signal go through independent channel realizations

39 This will be reflected in the semi-analytical performance evaluation of Chapter 5. 
for which the short-term delay profile may be very different. For a given channel realization, interferer phase could thus be important. But, in contrast to the static channel $D S L$ application of $[196,197]$ where interferer timing phase has a significant effect, the time-varying nature of the indoor fading channel makes the effect of changing a fixed interferer phase negligible when averaged over a large number of channel realizations. However, performance could probably be improved if interferer timing phase could be controlled and continuously optimized.

\subsubsection{Number of forward filter taps}

Increasing the number $L$ of $\frac{T}{2}$-spaced forward filter taps will improve average BER performance until $L$ is such that the equalizer span starts exceeding the total symbol dispersion. With $L I=L-1$ precursor taps and one central tap, it is observed from Figure 3.7 that, in the presence of a single cochannel interferer at a $10 \mathrm{~dB}$ SIR and with the rms delay spread equal to $0.25 T$, performance at an average BER of $10^{-3}$ improves by $16.3 \mathrm{~dB}$ the time-rectangular pulse and by $14.5 \mathrm{~dB}$ for the $50 \%$ SRC pulse when going from $L=l$ to $L=3$. Increasing $L$ from 3 to 5 then yields a gain of $1 \mathrm{~dB}$ for the timerectangular pulse and only $0.2 \mathrm{~dB}$ for the $50 \%$ SRC pulse. For the latter pulse, another $0.4 \mathrm{~dB}$ is gained when going from $L=5$ to $L=I l$ while the corresponding improvement is negligible for the time-rectangular pulse. With $L=3$ and $L=5 \frac{T}{2}$-spaced taps, the equalizer span is respectively $T$ and $2 T$. With an rms delay spread $\sigma_{\mathrm{d}}$ equal to $0.25 \mathrm{~T}$ (i.e a signaling rate of $10 \mathrm{Mbits} / \mathrm{s}$ for a typical $25 \mathrm{~ns} \mathrm{rms}$ delay spread), the exponential delay profile $\phi(\tau)=\frac{1}{\sigma_{d}} e^{-\frac{\tau}{\sigma_{d}}}$ is down to $1.83 \%$ of its peak value (i.e. $\tau=0$ ) at $\tau=\tau$ and down to $0.03 \%$ of its peak value at $\tau=2 T$. It is thus reasonable to expect that using $L>3$ taps will only yield a marginal further improvement and as complexity is a prime concern, $L=3$ will be used throughout most of the remainder of this thesis.

Splitting the $L$ forward filter taps into $L I$ precursor taps and $L 2>0$ postcursor taps operating in parallel with the feedback taps has been shown [176] to improve rejection of cochannel interference. From Figure 3.8, it appears that, for the $L=3$ and $L=5$ cases, $L 1>2$ is required to avoid a performance degradation with respect to the $L 2=0$ case as $L I=1$ does not provide a sufficient number of precursor taps. However, splitting $L=11$ 
taps into $L I=5$ precursor taps and $L 2=5$ postcursor taps can yield modest improvement over the $L 2=0$ case, particularly for large $\frac{E_{*}}{N_{1}}$ where cochannel interference is a more important impairment than white gaussian noise. Hence, if complexity were not of prime concern, it could be attractive to use $L \gg 5$ forward filter taps with the extra taps used as postcursor taps, not only to improve cochannel interference rejection but also to reduce dependency on sampling phase.

\subsubsection{Delay spread (signaling rate)}

The performance gains that can be achieved (with $L=3$ taps) by exploiting the implicit diversily of a frequency-selective channel are shown in Figure 39 for $\mathbf{M = 2}$ antennas and in Figures 3.10 for $M=3$ antennas. At an average BER of $10^{-3}, 6.1 \mathrm{~dB}$ with $M=2$ and $2.9 \mathrm{~dB}$ with $\mathrm{M}=3$, can be gained by increasing the rms delay spread from $10^{-7} \mathrm{~T}$ to $0.25 \mathrm{~T}$. As a more practical example, note that by going from $\sigma_{d}=0.05 T$ to $\sigma_{d}=0.25 T$, gains (at $B E R=10^{-3}$ ) of $1.9 \mathrm{~dB}$ and $1.4 \mathrm{~dB}$ are obtained for $M=2$ and $M=3$ antennas respectively. For a typical mean rms delay spread of $\sigma_{d}=25 n s$, this corresponds to increasing the data rate from $2 \mathrm{Mbits} / \mathrm{s}$ to $10 \mathrm{Mbits} / \mathrm{s}$. Note, however, that most of the gain occurs in going from $\sigma_{d}=0.05 T$ to $\sigma_{d}=0.10 T$ which thus seems to be the minimum value of $\sigma_{d}$ (i.e $4 \mathrm{Mbits} / \mathrm{s}$ ) for which most of the implicit diversity gain is obtained. A very small loss, particularly for large $\frac{E_{b}}{N_{0}}$, is then incurred by increasing $\sigma_{d}$ to $0.50 \mathrm{~T}$ (i.e. $20 \mathrm{Mbits} / \mathrm{s}$ ). Hence, with $L=3$ forward filter taps, the optinnum range of data rates corresponds ${ }^{40}$ to $4 \mathrm{Mbits} / \mathrm{s}$ to $20 \mathrm{Mbits} / \mathrm{s}$. Beyond this point however, performance quickly degrades. In practice, the degradation is probably even more severe than predicted by Figures $3.9 \mathrm{~b}$ and $3.10 \mathrm{~b}$ as the assumption of correct feedback decisions may no longer hold for those larger values of $\sigma_{d}$ such as $T$ and 2T. This situation could be alleviated by using more forwand filter taps (i.e. greater complexity) in which case the optimum range of $\sigma_{d}$ will tend to increase. Operating in more severe delay spread may however be more demanding in the form of longer exyalizer training sequences.

40 A higher rms delay spread in sbsolute terms will reduce this optimum range e.g. for $\sigma_{d}=50$ ns, the range becomes 2 Mbits/s to 10 Mbits/s. 


\subsubsection{Signal-to-interference ratio}

The influence of varying the signal-to-interference ratio $\frac{S}{T}$ is shown in Figures 3.11 for an uncoded system with $M=2$ and $M=3$ antennas and in Figure 3.12 for an $M=2$ anten:a system with convolutional coding and soft-decision Viterbi decoding. For the uncoded systems, average BER performance is found to be virtually independent (even asymptotically) of $\frac{S}{T}$ as long as $\frac{S}{T}>20 \mathrm{~dB}$. When coding is used with $\mathrm{M}=2$ antennas, the same is true for $\frac{S}{T}>15 d B$ and decreasing the $\frac{S}{T}$ ratio to $10 \mathrm{~dB}$ then degrades performance only slightly. However, for lower $\frac{S}{T}$, the coded system then degrades more quickly, in relative terms, than the uncoded cases, particularly with $M=3$. It thus appears that the coded system can tolerate somewhat lower $\frac{S}{T}$ ratios before a non-negligible performance degradation occurs at which point however coded system performance then degrades more quickly. In addition, the most probable breakdown of the correct feedback decisions assumption at $\frac{S}{T}=5 d B$ will once again make the actual degradation more severe than predicted by this analysis.

\subsubsection{Coding vs. space diversity}

The relative merit of increasing the space diversity order from 2 to 3 or adding coding to the $M=2$ system is shown in Figure 3.13 for $\frac{S}{l}$ values of infinity (i.e. $N=0$ ), $10 \mathrm{~dB}$ and $5 \mathrm{~dB}$. In order to maintain the same rate of information throughput and the same bandwidth, rate $\frac{1}{2}$-coded QPSK is compared to uncoded BPSK. At an average BER of $10^{-3}$, the $M=2$ coded system outperforms the uncoded $M=3$ system by $1 \mathrm{~dB}$ for both $\frac{S}{T}=\infty$ and $\frac{S}{T}=10 d B$ and by $0.5 \mathrm{~dB}$ for $\frac{S}{T}=5 d B$. These differences increase asymptotically and it thus appears that convolutional coding with soft-decision Viterbi decoding can be a valid alternative to increasing the space diversity order, albeit somewhat less so as $\frac{S}{T}$ falls below $10 \mathrm{~dB}$. Of course, this is true under the condition that the channel could be fully interleaved. As mentioned earlier, the latter is impractical in terms of complexity and delay for the indoor channel. Simulation results in Chapters $\mathbf{4}$ and 5 will then determine if the absence of interleaving degrades coded performance sufficiently to make coding unattractive with respect to increasing the space diversity order. 


\subsubsection{QPSK vs. BPSK}

The effect of changing the rms delay spread on QPSK performance is shown in Figure 3.14 for $M=2$ antennas and in Figure 3.15 for $M=3$ antennas. Just as for BPSK, it appears that with QPSK, $\sigma_{d} \geq 0.10 T$ is required to get the most gain out of the implicit diversity effect with $L=3$ forward filter taps and that the performance degradation

resulting from increasing symbol dispersion becomes severe only for $\sigma_{d} \geq 0.50 T$. In comparing BPSK and QPSK modulations, one should keep in mind that, for a given rate of information throughput i.e. for a given bit rate, the symbol period $r$ for BPSK becomes $2 T$ for QPSK. An important consequence of this is that BPSK requires twice as much bandwidth per user channel and hence, yields half as much circuit capacity (i.e. user channels per total system bandwidth). Therefore, with respect to symbol rate $1 / T$, a given $\sigma_{d}$ for QPSK becomes $2 \sigma_{d}$ for BPSK. In addition, since QPSK has twice the bandwidth efficiency of BPSK, it is clear that, if the system is to meet a certain bits/sec/Hz requirement, one may use a 0\% rolloff SRC pulse for BPSK and a $100 \%$ rolloff SRC pulse for QPSK. These changes are incorporated in the comparisons of Figures 3.16 and 3.17 where a QPSK curve with rms delay spread $\sigma_{d}$ is to be compared with a corresponding BPSK curve with rms delay spread $2 \sigma_{d}$. On the basis of the typical mean ms delay spread of $25 \mathrm{~ns}$, Figure 3.16 shows that, at an average BER of $10^{-3}$, BPSK outperforms QPSK at $4 \mathrm{Mbits} / \mathrm{s}$ by $4.1 \mathrm{~dB}$ for $\mathrm{M}=2$ and by 1.3 $\mathrm{dB}$ for $\mathrm{M}=3$. At $20 \mathrm{Mbits} / \mathrm{s}, \mathrm{BPSK}$ is still superior to $\mathrm{QPSK}$ by about $2.0 \mathrm{~dB}$ for $M=2$ and $0.9 \mathrm{~dB}$ for $M=3$. The advantage of BPSK over QPSK is reduced to $1 \mathrm{~dB}$ at $40 \mathrm{Mbits} / \mathrm{s}$ for $M=2$ while for $M=3$, the two modulations perform identically. If the cochannel interference is now eliminated as in Figure 3.17, at an average BER of $10^{-3}$, BPSK and QPSK perform identically at 4 and $20 \mathrm{Mbits} / \mathrm{s}$ for both $M=2$ and $M=3$. At 2 Mbits/s however, BPSK is slightly superior to QPSK at high $\frac{E_{b}}{N_{0}}$. Finally, at $40 \mathrm{Mbits} / \mathrm{s}, \mathrm{QPSK}$ is better than BPSK by $0.5 \mathrm{~dB}$ (at $B E R=10^{-3}$ ) for both $\mathrm{M}=2$ and $M=3$. It should be noted that if these results were interpreted in terms of the typical peak rms delay spread of $50 \mathrm{~ns}$, the data rates of $4 \mathrm{Mbits} / \mathrm{s}, 20 \mathrm{Mbits} / \mathrm{s}$ and $40 \mathrm{Mbits} / \mathrm{s}$ would respectively become $2 \mathrm{Mbits} / \mathrm{s}, 10 \mathrm{Mbits} / \mathrm{s}$ and $20 \mathrm{Mbits} / \mathrm{s}$.

It is thus clear that for low data (bit) rates, BPSK is superior to QPSK because of 
the absence of cross-rail interference caused by intersymbol and cochannel interference. As the data rate increases, QPSK eventually outperforms BPSK as the latter's higher symbol rate makes it more susceptible to the degradation caused by symbol dispersion. The transition point in terms of increasing data rate, i.e. beyond which QPSK is better than BPSK, is however higher for large $\frac{E_{b}}{N_{0}}$ (where ISI and CCI dominate) than for small $\frac{E_{6}}{N_{10}}$ (where AWGN dominates). It is also higher for $M=2$ antennas than for $M=3$ antennas when cochannel interference is significant; however, if cochannel interference is negligible, the transition point is similar for $M=2$ and $M=3$. 


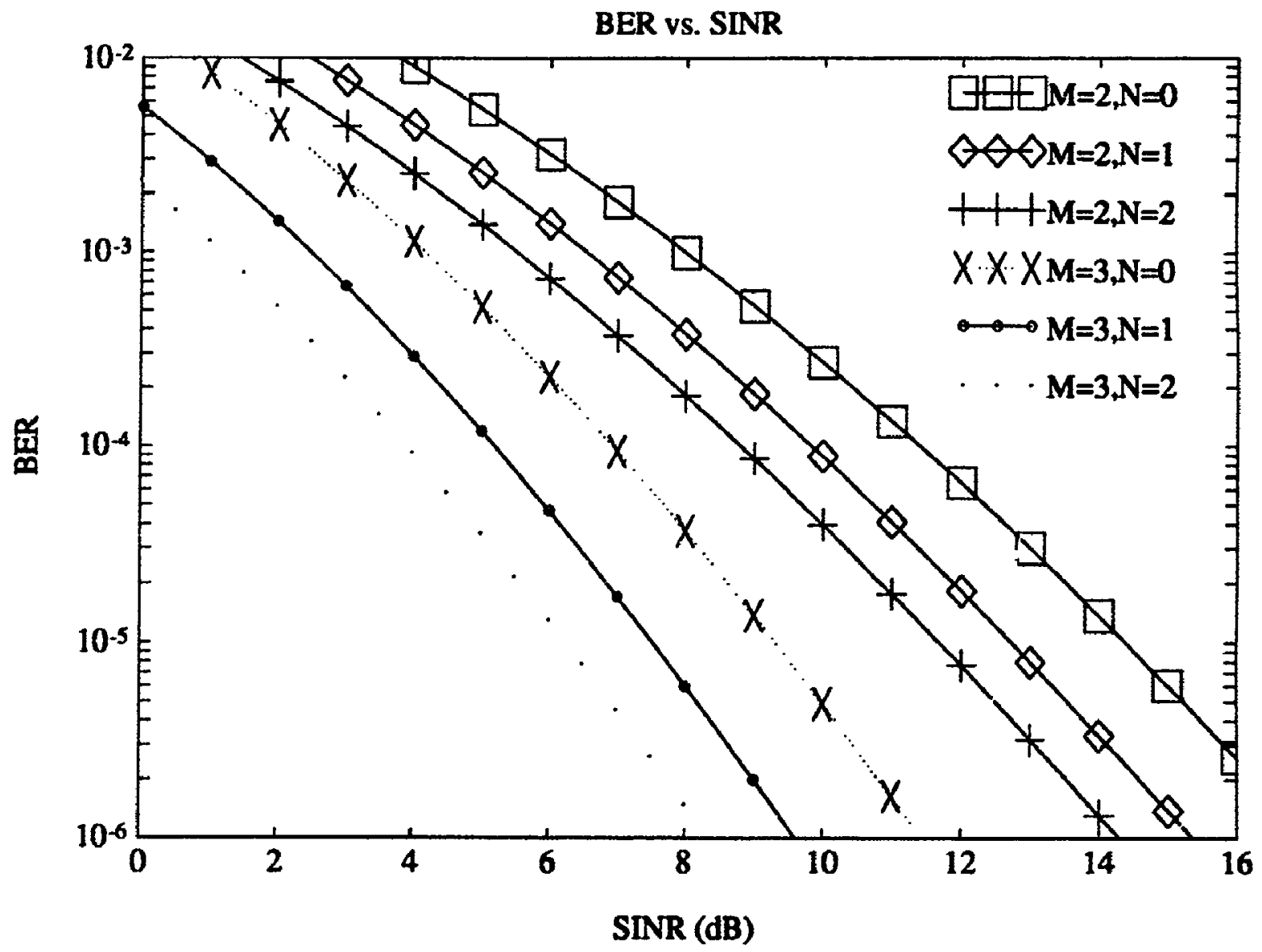

Figure 3.3 BPSK BER vs. SINR $\Gamma$. Interferer-to-noise ratio $\mathrm{E}_{\mathrm{Sn}} / \mathrm{NO}=1$ for $\mathrm{n}=1,2$, $t_{0}=0.0 T, t_{1}=0.125 T, t_{2}=0.25 T, \sigma_{d}=0.25 T$, rectangular pulse shape, $L=3, L 1=2, L 2=0$. 


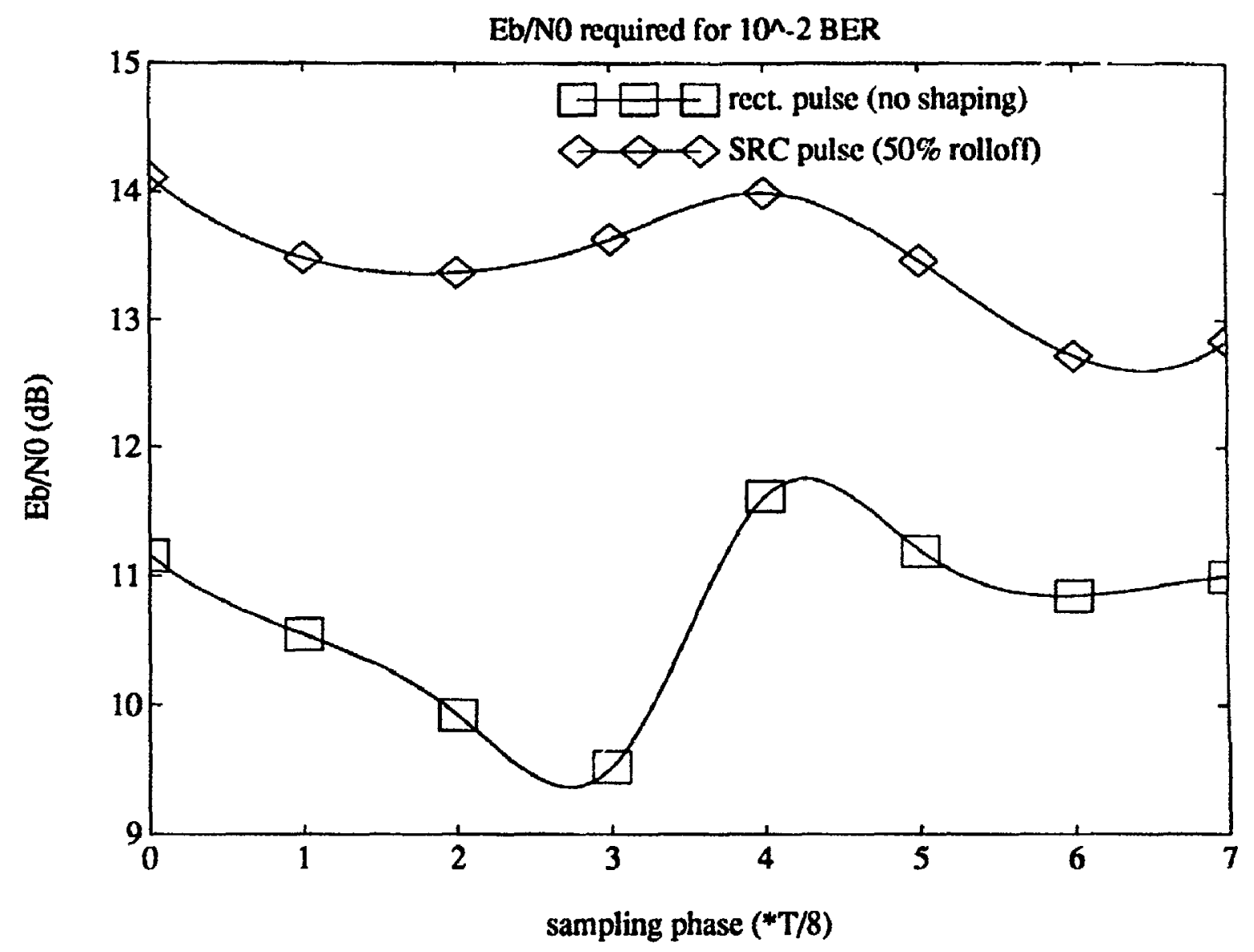

Figure 3.4 Effect of fixed sampling phase $t_{0}$. $M=1, N=0, L=3, L 1=2, L 2=0, \sigma_{d}=0.25 T$, BPSK. 
a)

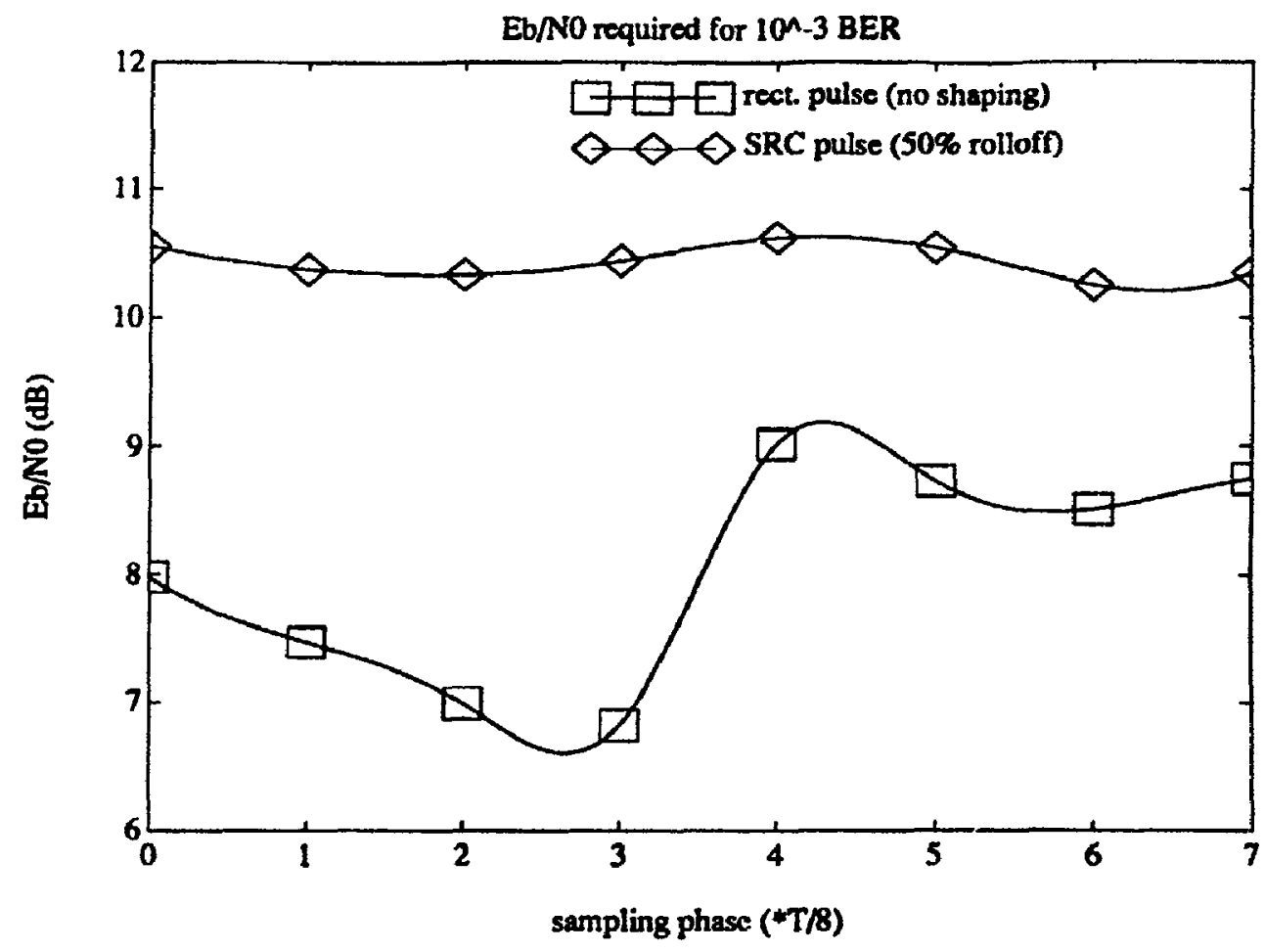

Eb/NO required for 10n-3 BER

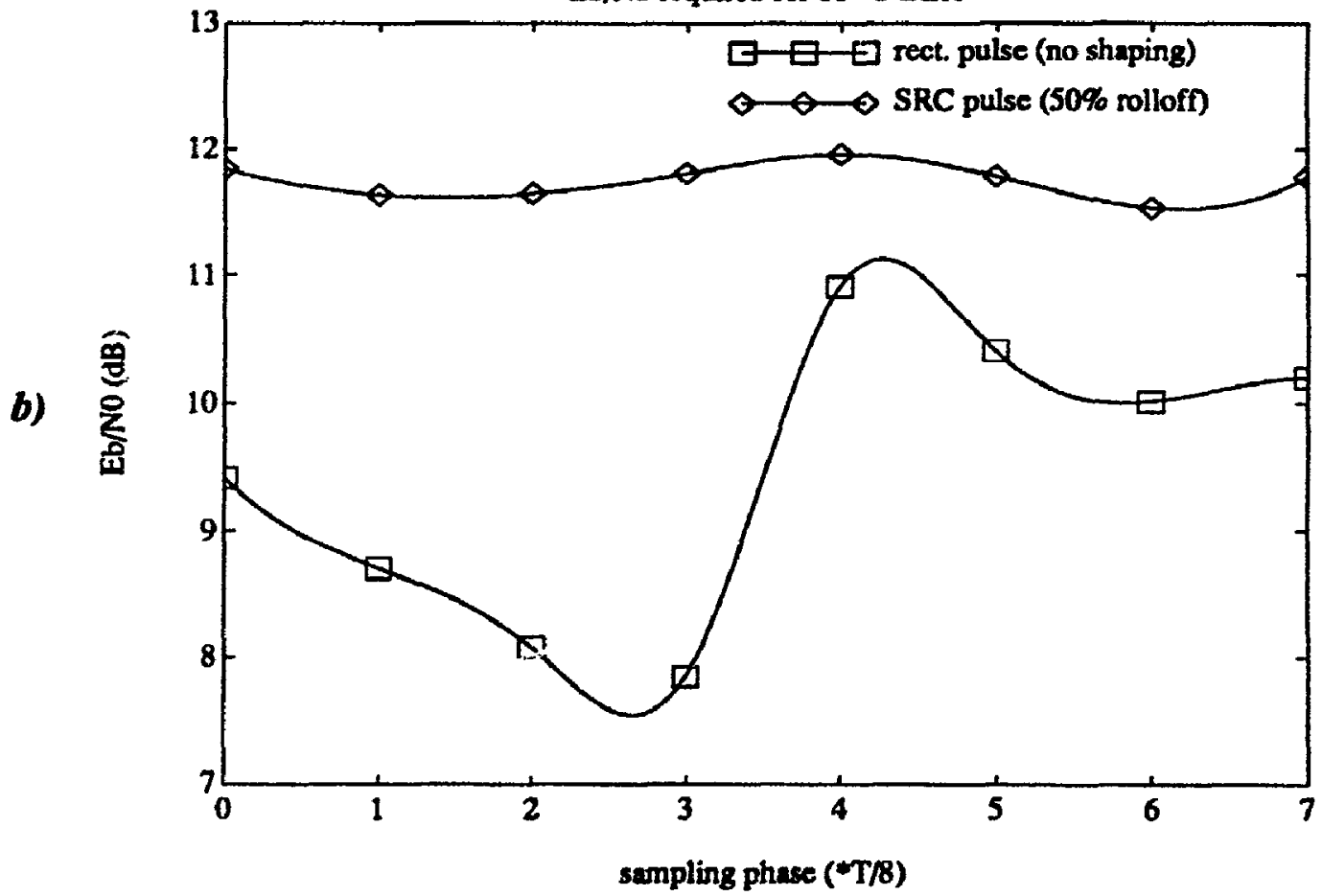

Figure 3.5 Effect of fixed sampling phase to.

$M=2, L=3, L 1=2, L 2=0, \sigma_{d}=0.25 T$, BPSK.

a) $N=0$ b) $N=1, S / I=10 \mathrm{~dB}, t_{1}=0.0 \mathrm{~T}$. 
$\mathrm{Eb} / \mathrm{NO}$ required for 10 . 3 BER

a)

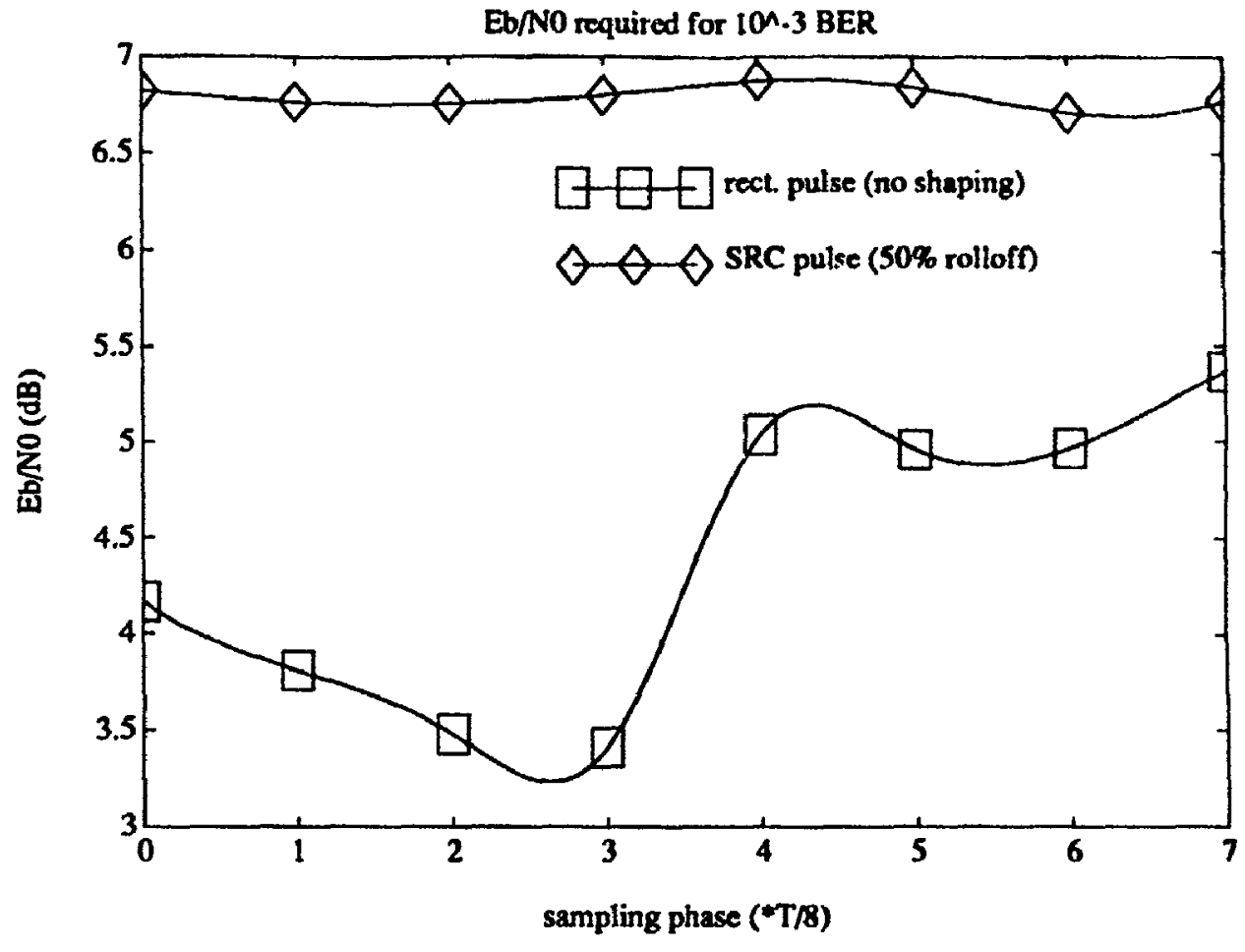

Eb/NO required for $10^{n}-3$ BER

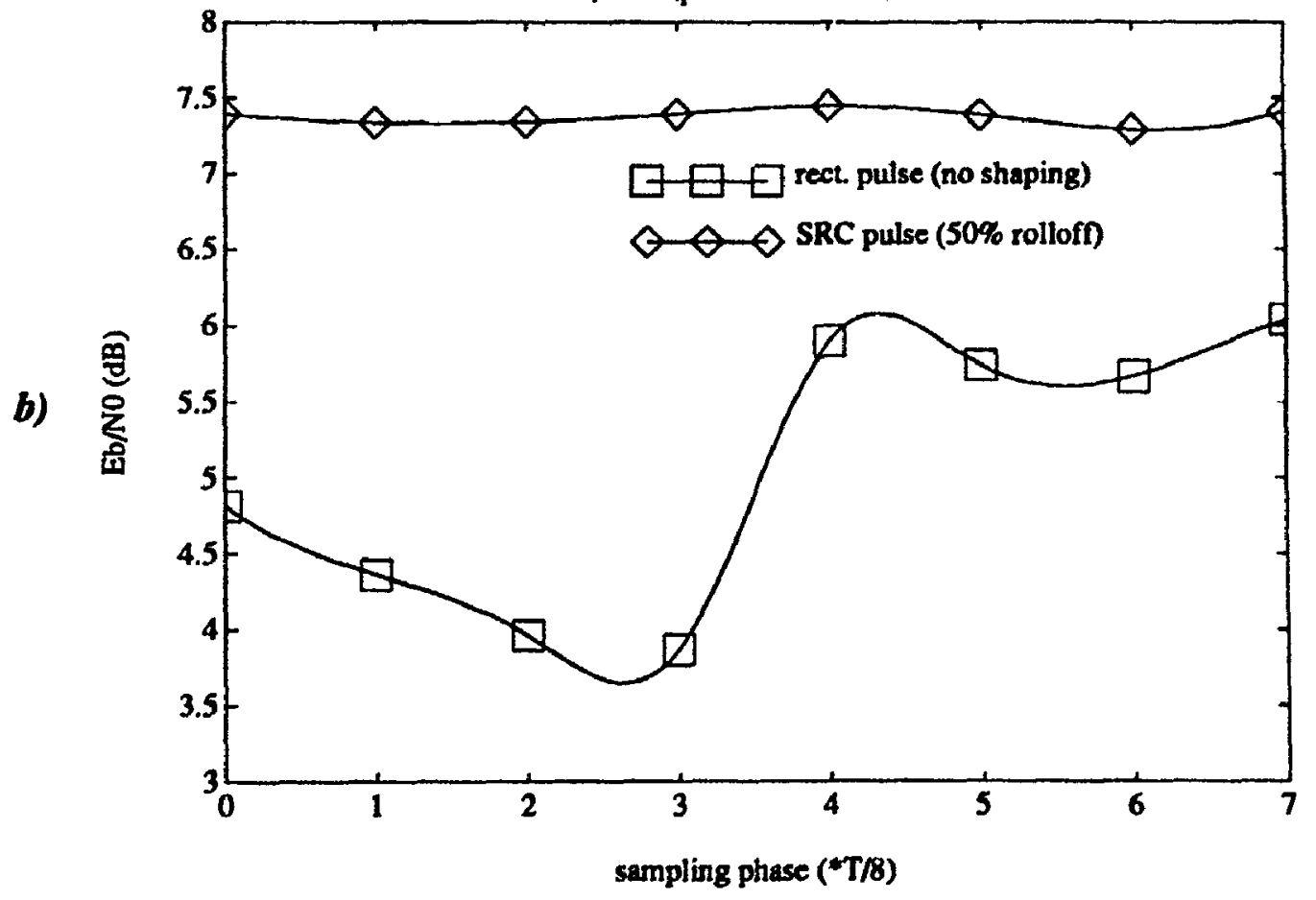

Figure 3.6 Effect of fixed sampling phase $t_{0}$. $M=3, L=3, L 1=2, L 2=0, \sigma_{d}=0.25 T$, BPSK.

a) $N=0$ b) $N=1, S / 1=10 \mathrm{~dB}, t_{1}=0.0 T$. 


\begin{tabular}{|c|c|c|}
\hline \multirow{2}{*}{$\begin{array}{c}\mathrm{M}: \text { \# of antennas } \\
\mathrm{N}: \# \text { of cochannel } \\
\text { inteferers }\end{array}$} & \multicolumn{2}{|c|}{ Pulse shape } \\
\hline & $50 \%$ SRC & Time-rectangular \\
\hline $\mathrm{M}=1, \mathrm{~N}=0^{*}$ & $1.40 \mathrm{~dB}$ & $2.04 d B$ \\
\hline$M=2, N=0$ & $0.37 d B$ & $1.98 \mathrm{~dB}$ \\
\hline $\mathrm{M}=2, \mathrm{~N}=1$ & $0.39 \mathrm{~dB}$ & $3.06 \mathrm{~dB}$ \\
\hline $\mathbf{M}=3, \mathbf{N}=\mathbf{0}$ & $0.17 \mathrm{~dB}$ & $1.96 \mathrm{~dB}$ \\
\hline $\mathbf{M}=3, \mathbf{N}=1$ & $0.18 \mathrm{~dB}$ & $2.17 \mathrm{~dB}$ \\
\hline
\end{tabular}

* This case (figure 3.4) corresponds to a $10^{-2}$ BER as irreducible BER's in the range of $10^{-3}$ to $10^{-4}$ were observed in this case for the worst sampling phase.

Table 3.1: Sampling phase sensitivities in terms of $\frac{E_{b}}{N_{\mathrm{v}}}$ required for $10^{-3}$ average BER. These results correspond to Figures 3.4, 3.5 and 3.6. 

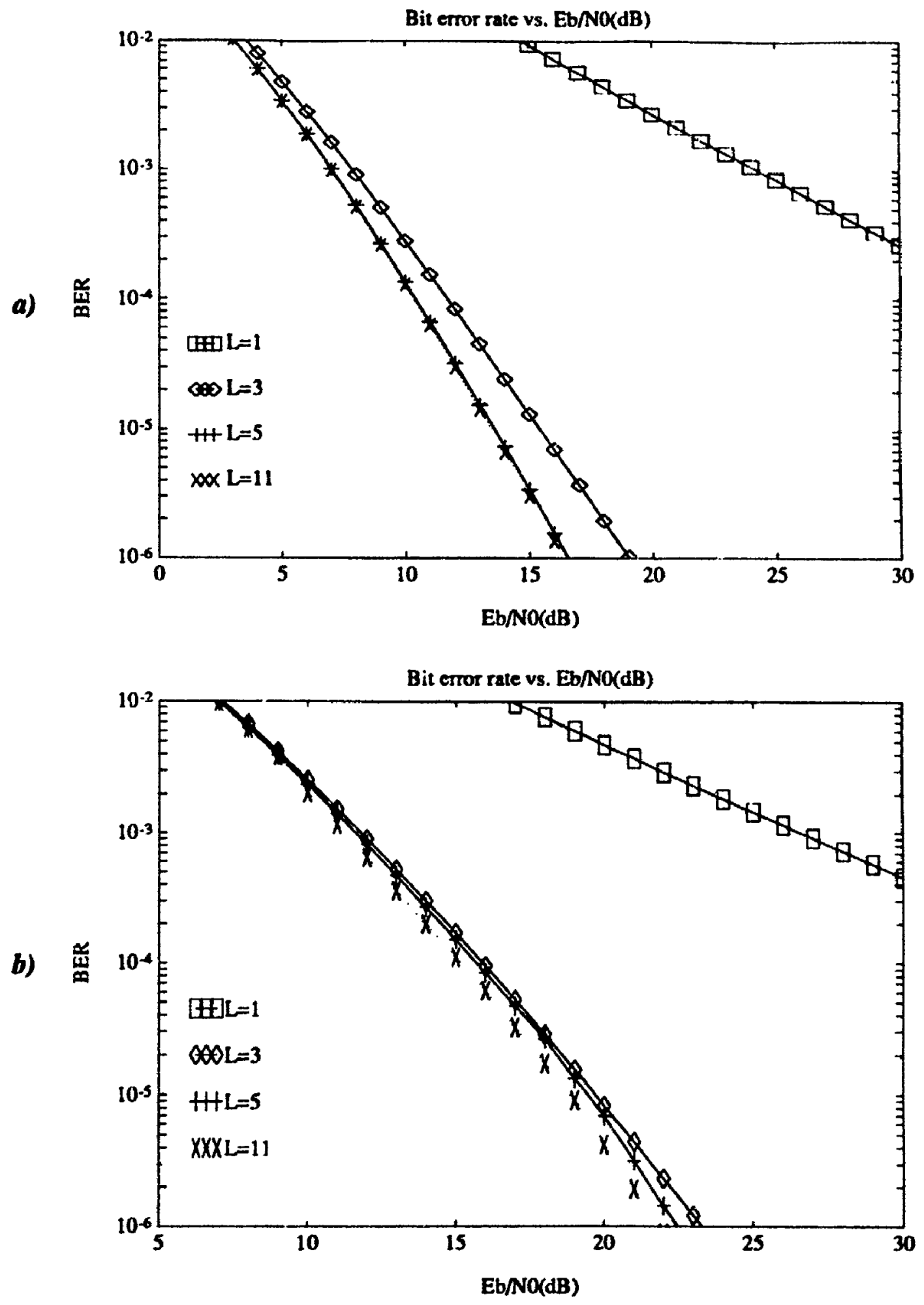

Figure 3.7 Effect of number of forward filter taps $L$. $M=2, N=1, L 1=L-1, L 2=0, t_{0}=0.375 T, t_{1}=0.0 T, \sigma_{d}=0.25 T$, BPSK.

a) Time-rectangular pulse b) $50 \%$ SRC pulse. 


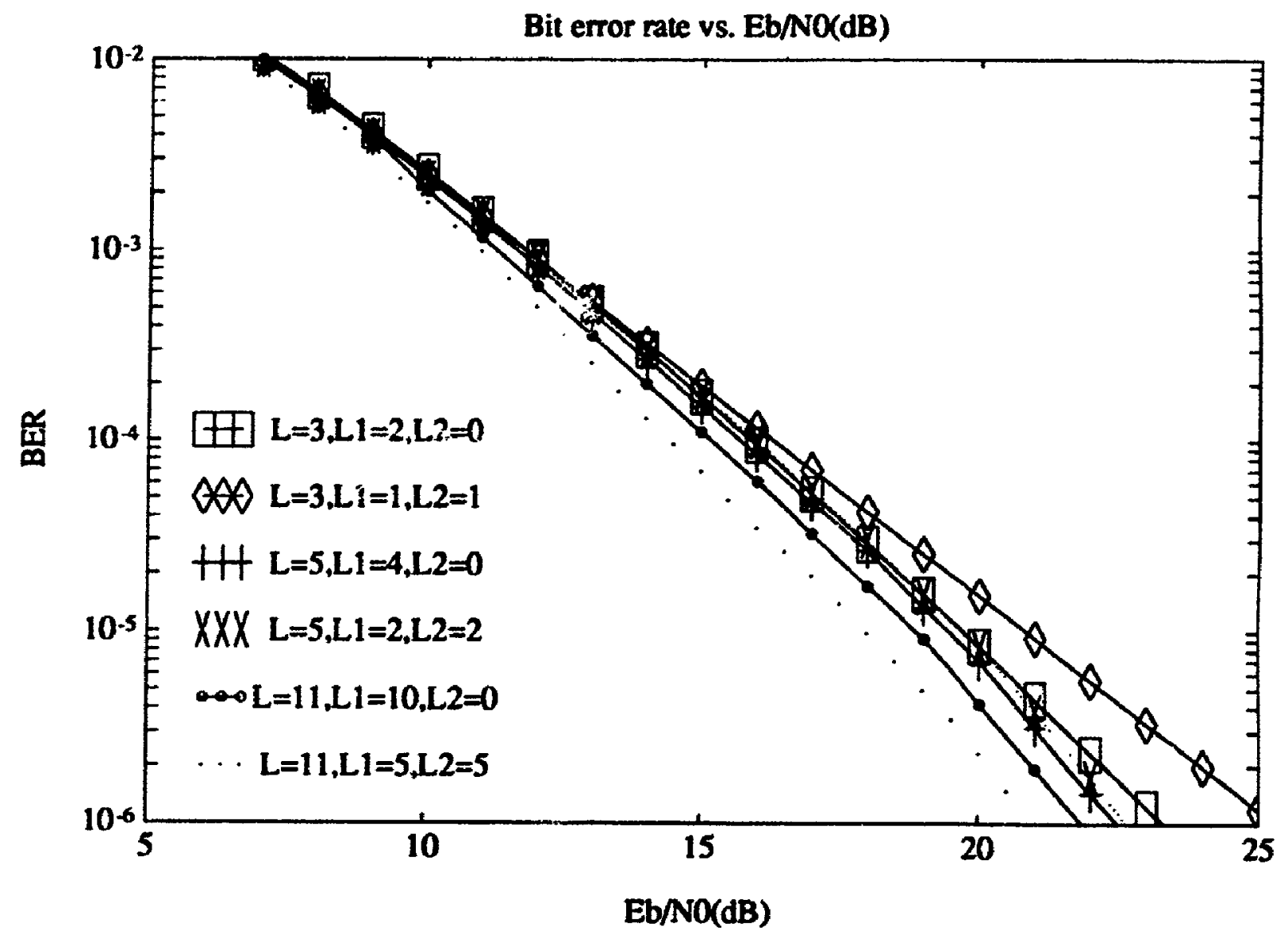

Figure 3.8 Efrect of splitting forward filter taps into $L I$ precursor taps and $L 2$ postcursor taps. $M=2, N=1, t_{0}=0.375 T, t_{1}=0.0 T, \sigma_{d}=0.25 T, 50 \%$ SRC pulse, BPSK. 

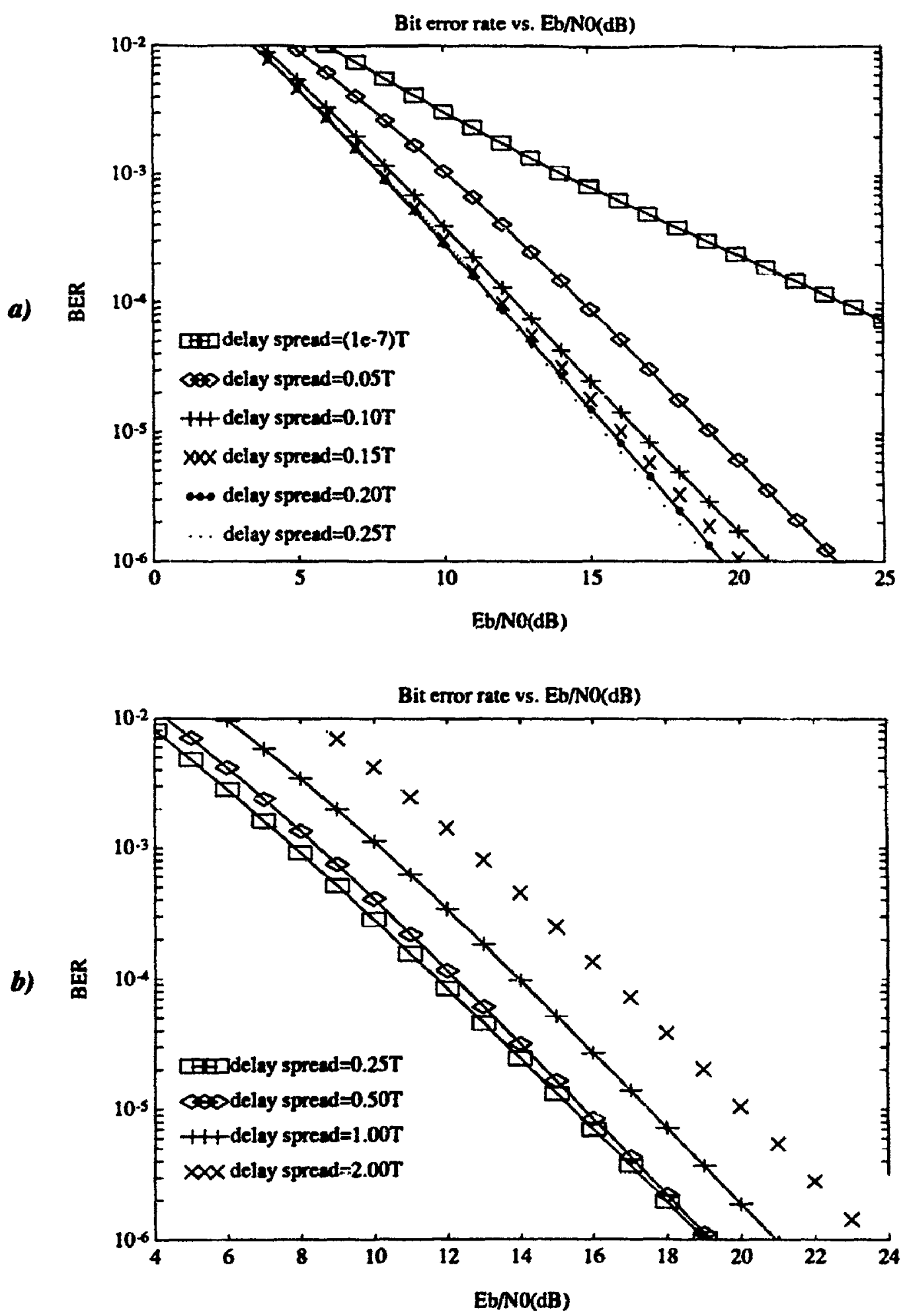

Figure 3.9 Effect of varying rms delay spread $\sigma_{\mathrm{d}}$. $M=2, N=1, S / I=10 \mathrm{~dB}, t_{1}=0.0 T$, time-rectangular pulse, $L=3, L 1=L-1, L 2=0, t_{0}=0.375 T$, BPSK. 

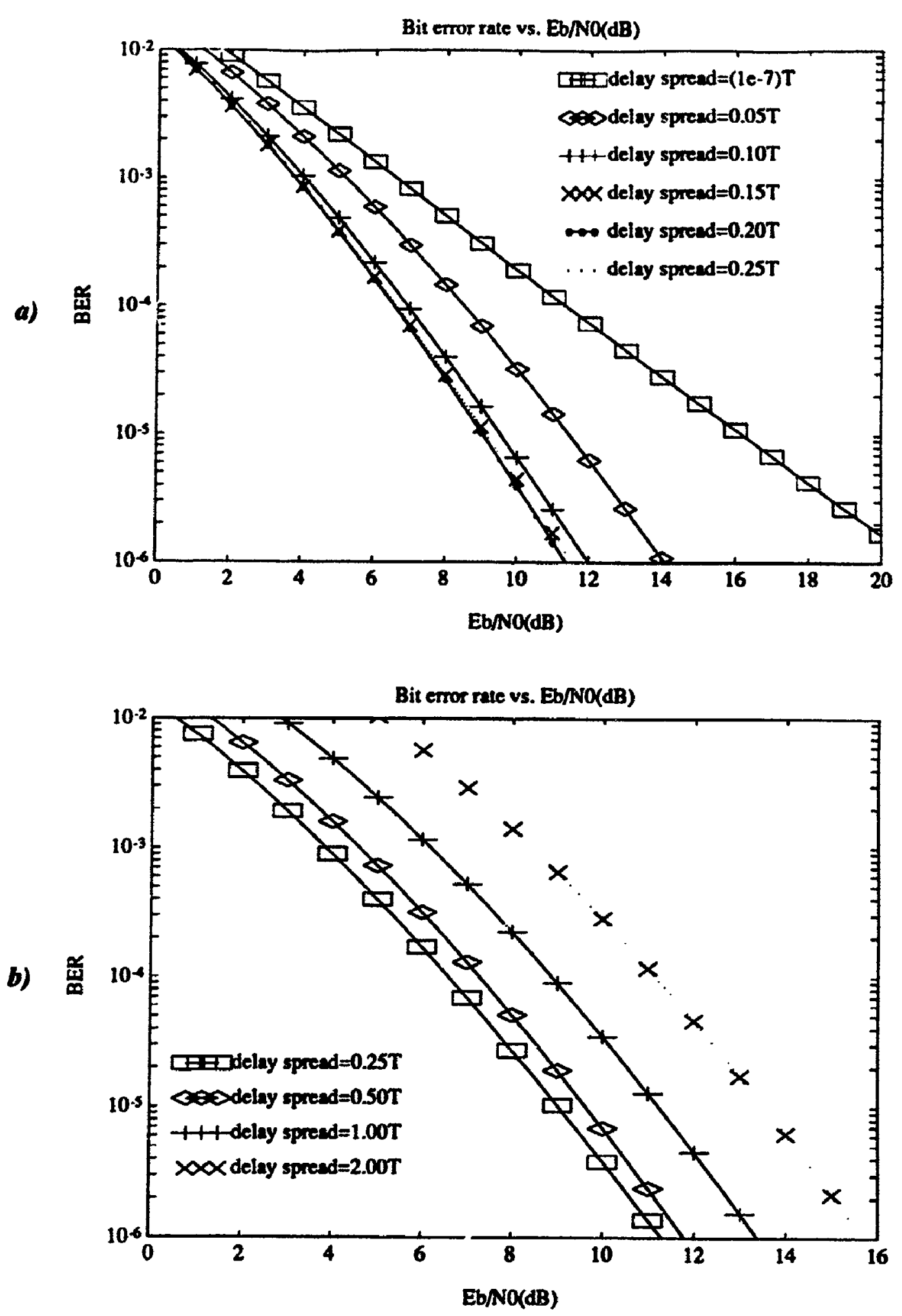

Figure 3.10 Effect of varying rms delay spread $\sigma_{\text {d }}$. $M=3, N=1, S / I=10 \mathrm{~dB}, t_{1}=0.0 \mathrm{r}$, time-rectangular pulse, $L=3, L 1=L-1, L 2=0, t_{0}=0.375 T$, BPSK. 

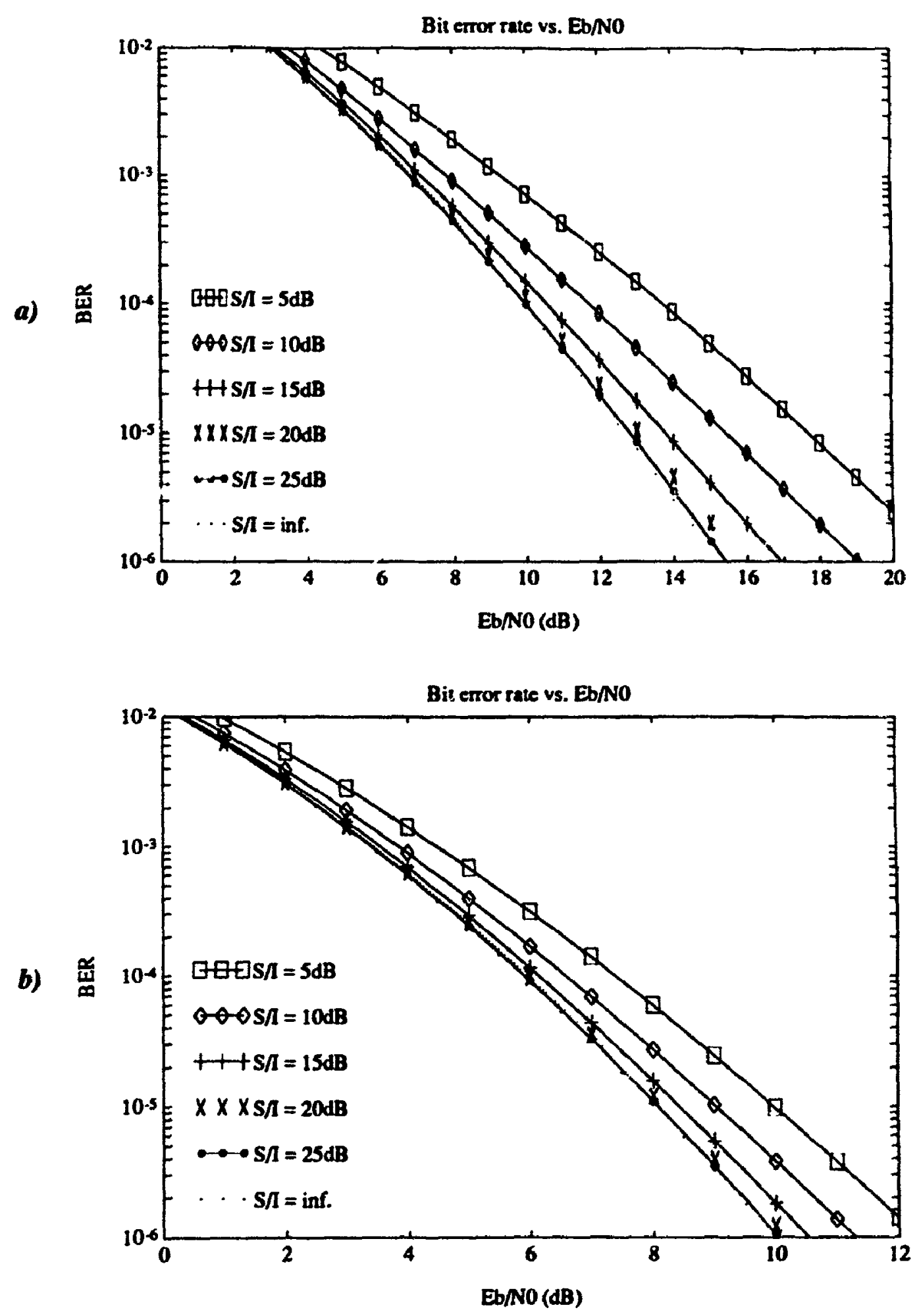

Figure 3.11 Effect of varying S/I ratio.

$N=1, t_{1}=0.0 T, \sigma_{d}=0.25 T$, time-rectangular pulse, $L=3$,

$L 1=L-1, L 2=0, t_{0}=0.375 T$, BPSK. a) $M=2$ b) $M=3$. 


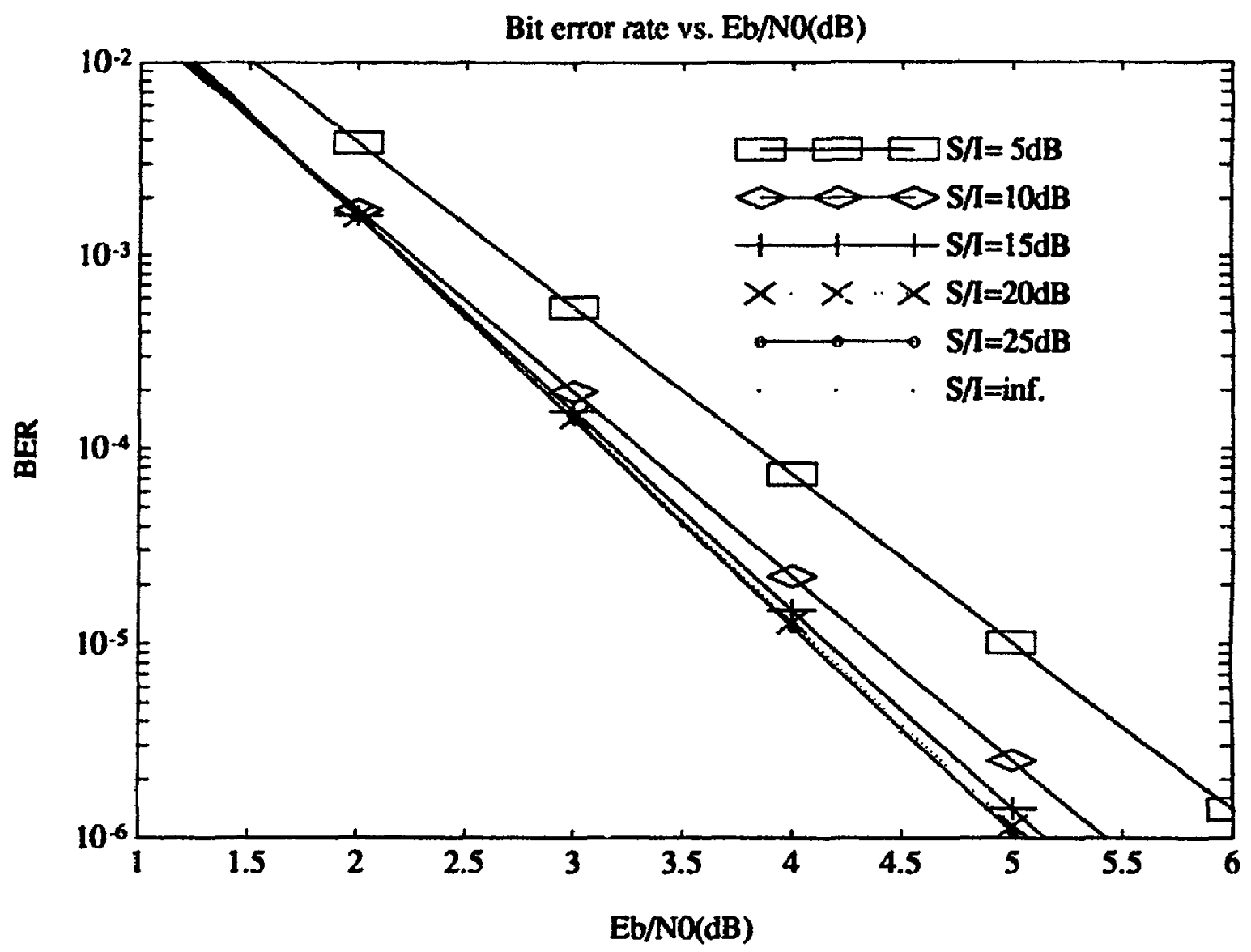

Figure 3.12 Effect of varying $\mathrm{S} / \mathrm{I}$ ratio for coded system. $M=2, N=1, t_{1}=0.0 T, \sigma_{d}=0.25 T$, time-rectangular pulse, $L=3, L 1=L-1, L 2=0$, $t_{0}=0.375 T$, BPSK. 


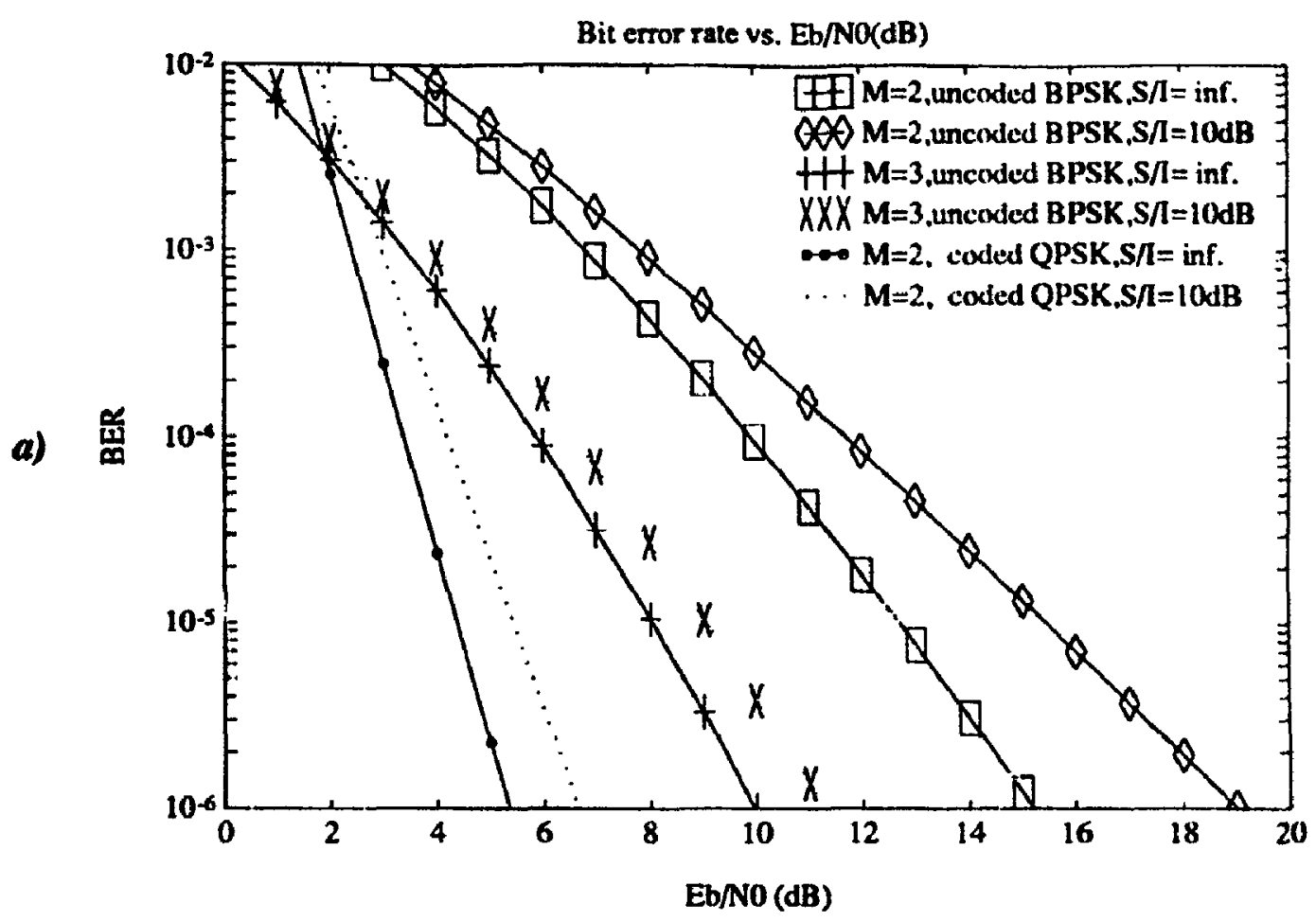

Bit error rate vs. Eb/NO(dB)

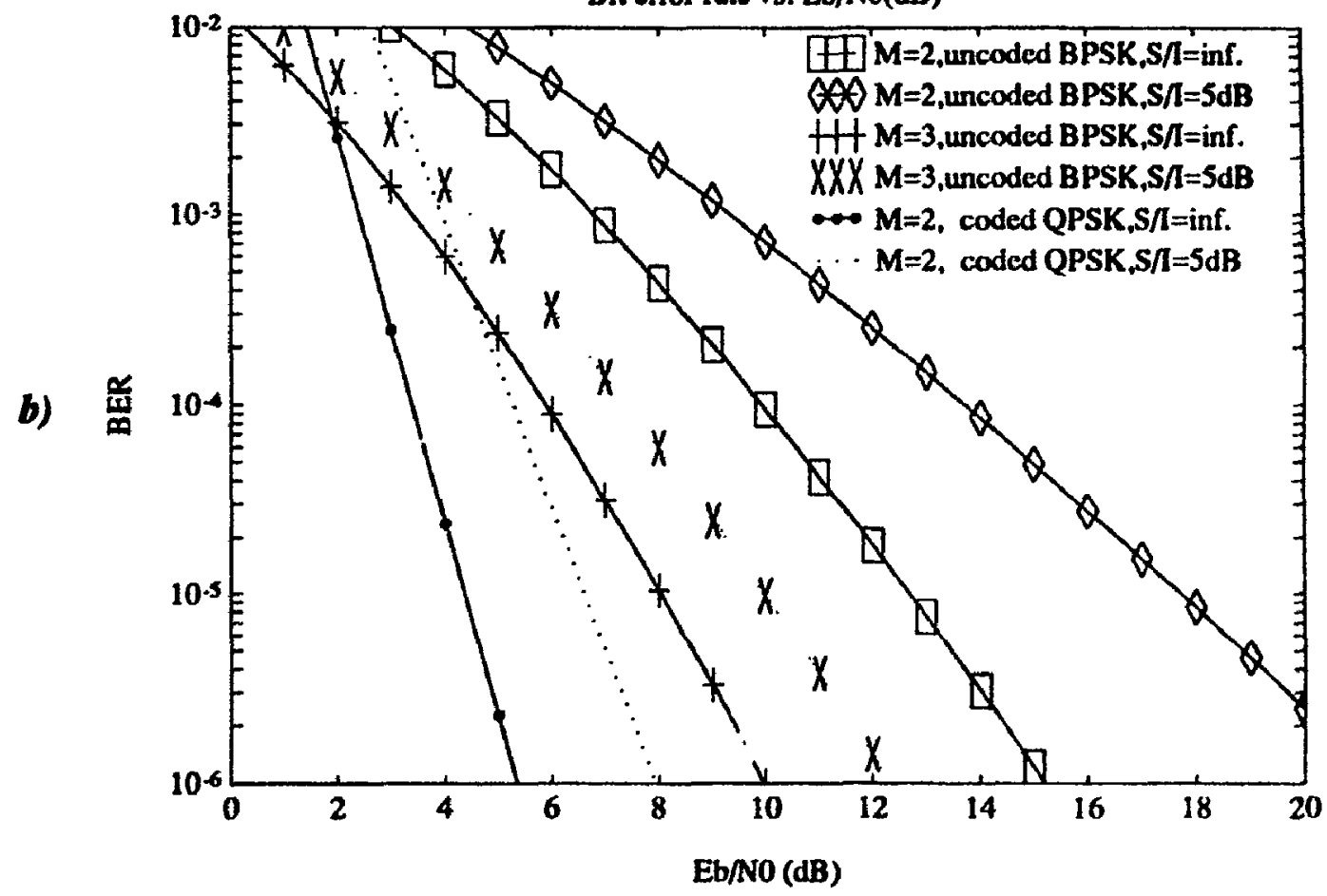

Figure 3.13 Coding vs. space diversity.

$N=1, t_{1}=0.0 T, \sigma_{d}=0.25 T$, time-rectangular pulse, $L=3, L 1=L-1, L 2=0, t_{0}=0.375 T$. 
Bit error rate vs. Eb/NO(dB)

a)
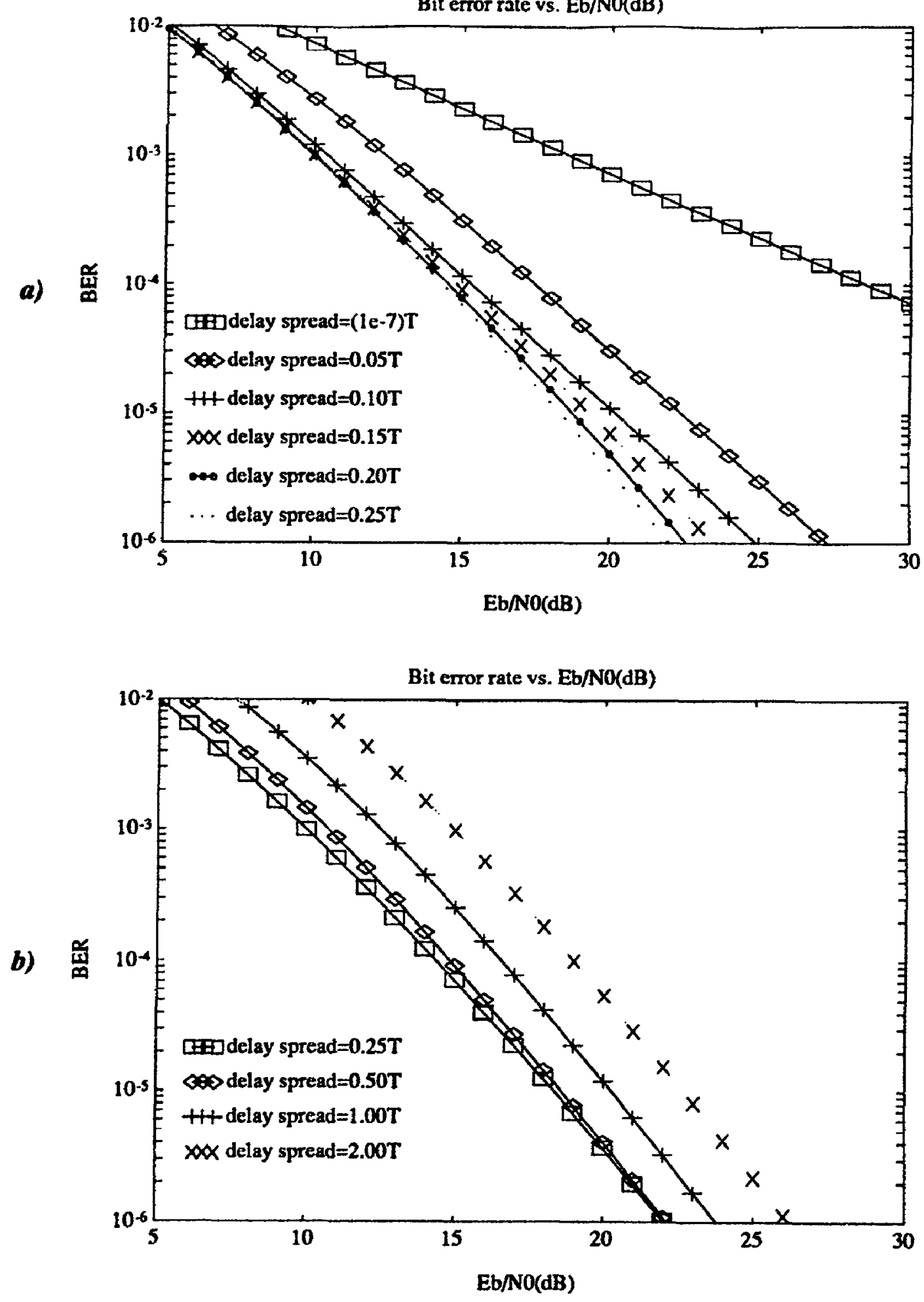

Figure 3.14 Effect of varying rms delay spread $\sigma_{\mathrm{d}}$.

$M=2, N=1, S / 1=10 \mathrm{~dB}, t_{1}=0.0 \mathrm{~T}$, time-rectangular pulse, $L=3, L 1=L-1, L 2=0, t_{0}=0.375 T$, QPSK. 
Bit error rate vs. Eb/NO(dB)
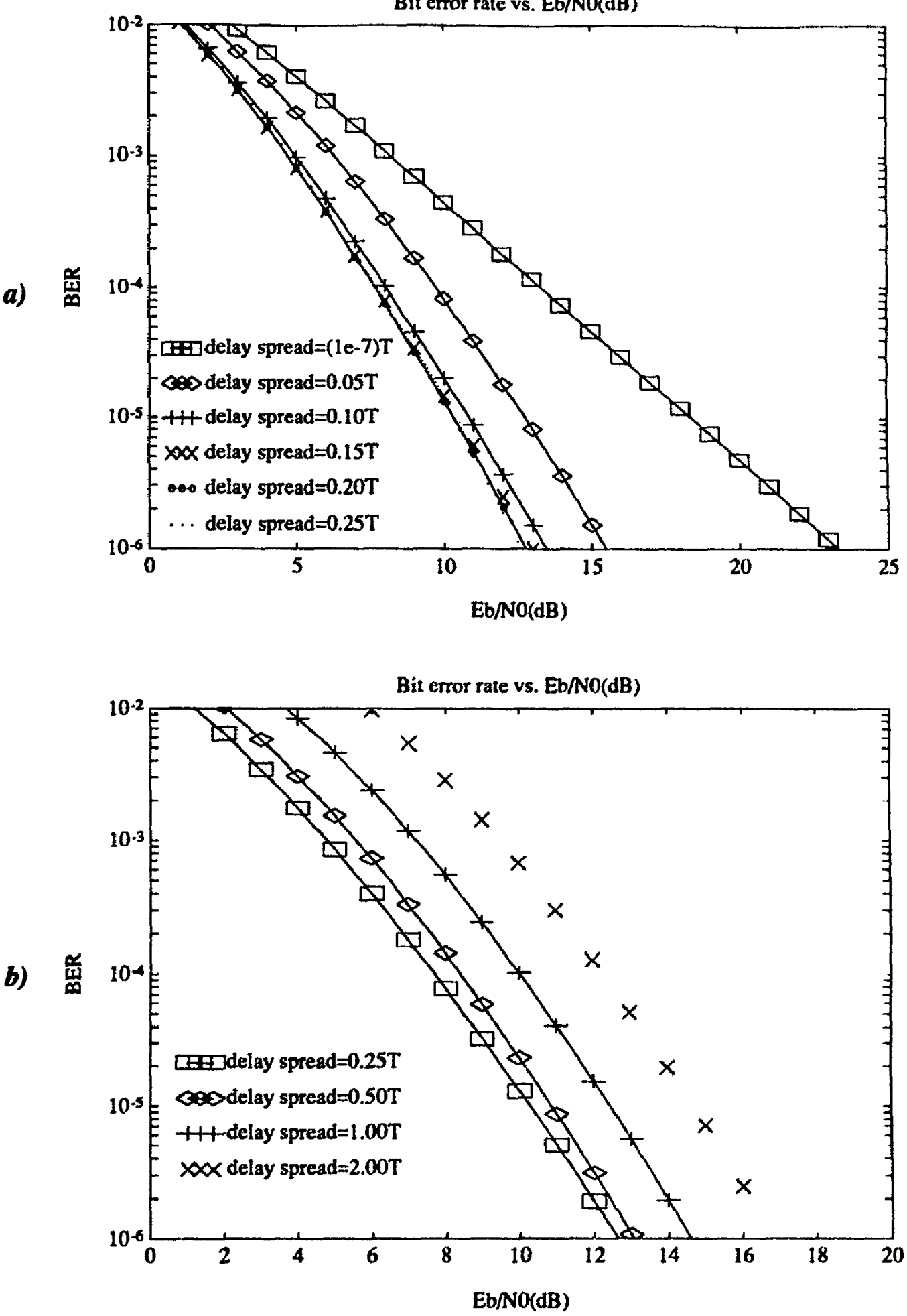

Figure 3.15 Effect of varying rms delay spread $\sigma_{\mathrm{d}}$.

$M=3, N=1, S / I=10 \mathrm{~dB}, t_{1}=0.0 T$, time-rectangular pulse, $L=3, L 1=L-1, L 2=0, t_{0}=0.375 T$, QPSK. 

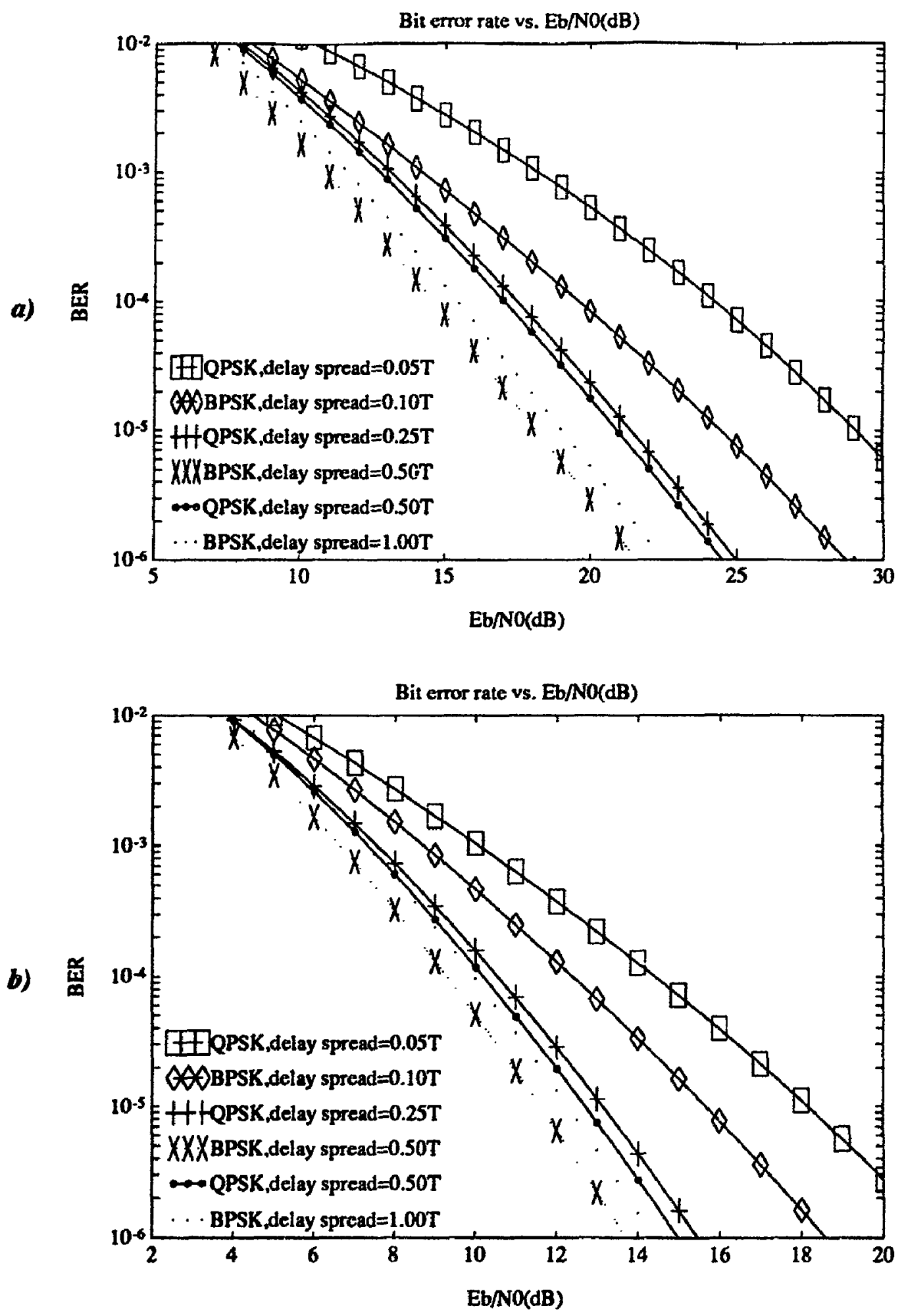

Figure 3.16 QPSK vs. BPSK. $\quad$ a) $M=2$ b) $M=3$. $N=1, S / L=10 d B, t_{1}=0.0 T, L=3, L 1=L-1, L 2=0, t_{0}=0.375 T$. QPSK uses 100\% SRC pulse; BPSK uses 0\% SRC pulse. 
Bit crror rate vs. Eb/NO(dB)
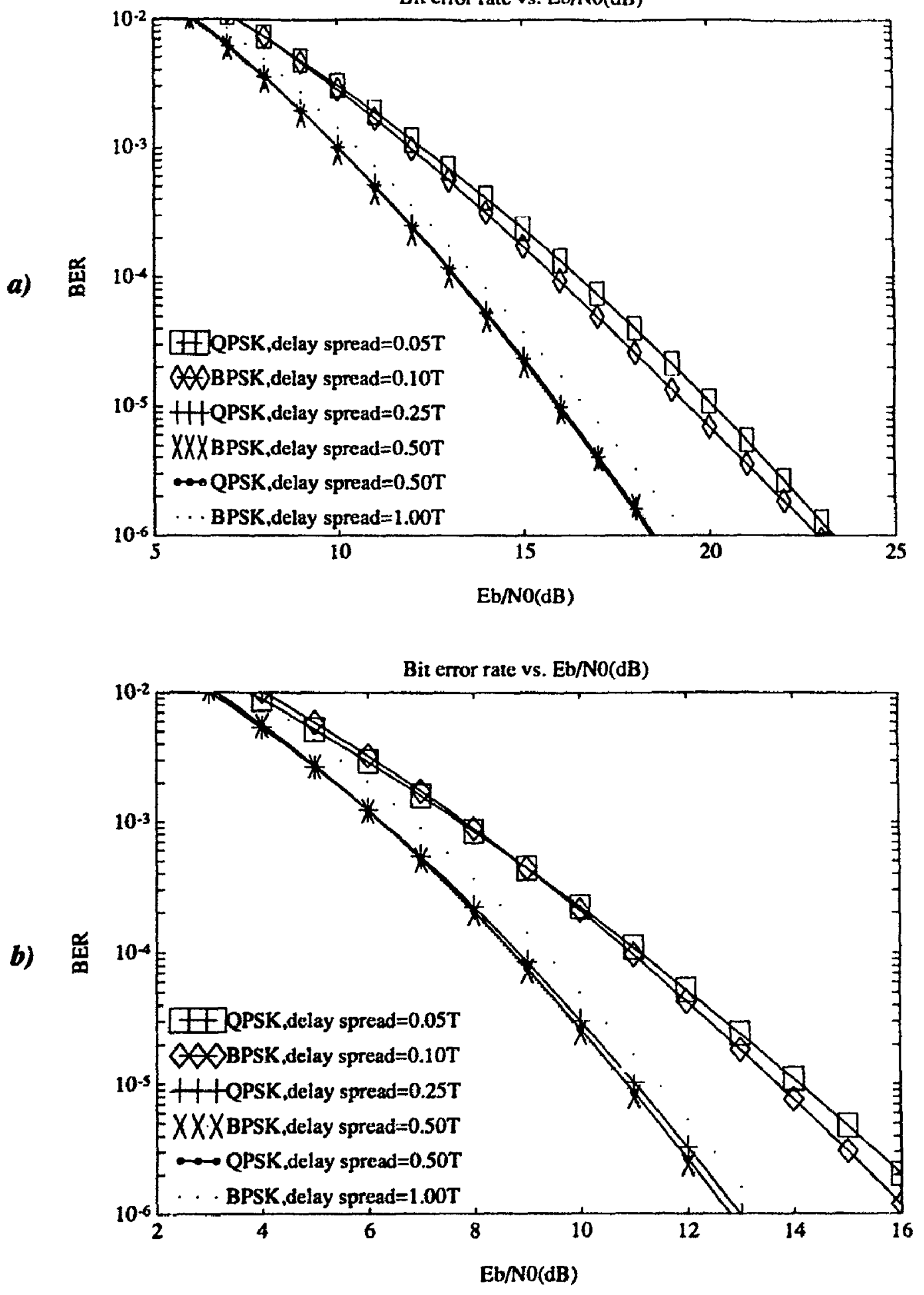

Figure 3.17 QPSK vs. BPSK. a) $M=2$ b) $M=3$.

$N=0, L=3, L 1=L-1, L 2=0, t_{0}=0.375 T$. QPSK uses $100 \%$ SRC pulse; BPSK uses 0\% SRC pulse. 


\subsection{Summary}

In this Chapter, the performance of optimum baseband diversity combining in conjunction with convolutional channel coding on a slowly-varying fading dispersive channel has been examined using a purely analytical approach. Some of the main conclusions are:

1. With a single receiver antenna $(\mathrm{M}=1)$, no cochannel interference $(\mathrm{N}=0)$, an $\mathrm{rms}$ delay spread $\sigma_{d}=0.25 T$ and only $L=3 \frac{T}{2}$-spaced forward filter taps, the $\frac{E_{b}}{N_{n}}$ required for an average BER of $10^{-3}$ is found to vary with a fixed receiver sampling phase by as much as $2 \mathrm{~dB}$ for a wide-bandwidth time-rectangular pulse and by 1.4 $\mathrm{dB}$ for a $50 \% \mathrm{SRC}$ pulse. The ability of an increased explicit space diversity order $M$ to reduce sampling phase sensitivity and to minimize its increase when cochannel interference is added is then found to be much stronger for the $30 \%$ SRC pulse than for the time-rectangular pulse. With $M=2$, sampling phase sensitivity only increases for the $50 \%$ SRC pulse from $0.37 \mathrm{~dB}$ with $\mathrm{N}=0$ to $0.39 \mathrm{~dB}$ with $\mathrm{N}=1$. However, the corresponding values for the time-rectangular pulse are 1.98 $\mathrm{dB}$ and $3.06 \mathrm{~dB}$.

2. For a given fixed receiver sampling phase and with $\mathrm{M}=2$ and $\sigma_{d}=0.25 T$, increasing the number of forward filter taps $L$ beyond 5 yields only a negligible Ferformance improvement unless some of the extra taps are used as postcursor taps, in which case modest gains are observed when interference is the dominant impairment. More than $90 \%$ of the improvement observed from $L=1$ to $L=5$ is obtained with $\mathrm{L}=3$.

3. With $L=3$ and $\frac{S}{I}=10 \mathrm{~dB}$, a data rate of at least $4 \mathrm{Mbits} / \mathrm{s}$ (based on a typical mean Ims delay spread of $\sigma_{d}=25 n s$ ) is required to significantly exploit the implicit diversity of the channel for both the $M=2$ and $M=3$ cases. Hence, performance degrades as the data rate is lowered from $4 \mathrm{Mbits} / \mathrm{s}$. Increasing the data rate from $4 \mathrm{Mbits} / \mathrm{s}$ then yields no significant degradation until it exceeds $20 \mathrm{Mbits} / \mathrm{s}$.

4. The $M=2$ coded system can tolerate lower $\frac{S}{I}$ values than the uncoded $M=2$ and $M=3$ systems before a non-negligible performance degradation occurs. However at 
very low $\frac{S}{T}$, coded system performance can degrade suddenly. In addition, under the assumption of ideal interleaving and with equal rates of information throughpur and equal bandwidths, the $M=2$ coded system is found to be superior to the $M=3$ uncoded system, albeit somewhat less so at lower $\frac{S}{I}$ ratios.

5. The transition point in terms of data rate beyond which QPSK is superior to BPSK is higher when interference dominates than when AWGN dominates. It is also higher with smaller $M$ when cochannel interference is significant. If cochannel interference is negligible, the transition point is similar for $M=2$ and $M=3$.

The analytical technique described in this Chapter is advantageous as it quickly yields a large number of results without resorting to any computation-intensive simulations. However, these analytical results are highly dependent on the selected meansquare value $\gamma^{2}$ of the gaussian interfering symbols. Coupled with the fact that in a high capacity frequency reuse portable radio system, cochannel interference is most often dominated by a single interfering source [117], this choice can affect the accuracy of the gaussian approximation of interference. In this sense, the selected value of $\gamma^{2}$ will affect the data rate beyond which QPSK is superior to BPSK. In addition, the results for low $S / I$ ratios (e.g. $5 \mathrm{~dB}$ ) are certainly optimistic as the assumption of correct feedback decisions may no longer hold in this case. As ideal interleaving is difficult to realize both in terms of complexity (for data rates in the Mbit/s range) and delay for a portable application, the coded results are also very optimistic and thus essentially represent a lower bound on achievable average bit error rate. Hence, while this fast analytical procedure is certainly useful in establishing trends or qualitative results, simulation techniques are required, albeit at the expense of increased computation time, to produce more reliable quantitative results. In addition, techniques which account for the short-term variations of the average delay profiles are required to evaluate performance accurately in terms of outage rate which is really the criterion of interest for portable applications. Such approaches will be discussed in the following two Chapters. 


\section{Chapter 4}

\section{PERFORMANCE EVALUATION VIA SIMULATION}

\subsection{Introduction}

In order to evaluate performance with all analytical assumptions relaxed and in particular to assess the effect of the non-interleaved channel, a bit-by-bit simulation was performed and is described in this Chapter. The simulations are implemented using the Block Oriented Systems Simulator (BOSS) ${ }^{T M 41}$, a software package providing an interactive environment for simulation-based analysis and design of communication systems which can be represented in a block diagram form where each block performs a signal processing operation. The main advantage of BOSS is that it assumes the responsibility of generating the simulation program, executing the simulation and displaying the results. A few examples of the various "modules" (i.e. block diagrams) used in implementing the desired system can be found in Appendix $\mathbf{C}$, along with a description of the block and of its relevant parameters. From the top-level modules shown in Appendix $\mathrm{C}$, there can be as many as five levels of complexity, i.e. modules within anodules, before one finds only the basic modules supplied by BOSS. In some cases however, e.g. with the convolutional coder and Viterbi decoder modules, it was simpler to actually write code directly instead of using the BOSS primitive modules ${ }^{42}$. As is commonly done, bandpass signals and systems are represented by their complex low-pass equivalents in order to reduce the simulations' sampling rate requirements. The latter was set to eight samples per symbol interval for all simulations.

4 Block Oriented Systems Simulator and BOSS are trademarks of Comdisco Systems, Inc.

42 The latest available version of BOSS, at the time of writing this thesis, provides more complex basic modules such as coders and decoders. 


\subsection{Channel model}

Most of the BOSS modules designed for the simulation (some of which are shown in Appendix C), although fairly complex, are generally self-explanatory. However, the channel model, as implemented in the frequency-selective Rayleigh fading (static) module, warrants further discussion.

There are both continuous and discrete methods [94] available for modeling the indoor radio channel. Continuous channel modeling (i.e. equation 3.3) is suitable for mathematical analysis as it uses the average received power at every delay of interest. Each impulse response $h_{n i}(\tau)$ is a sample of the channel between a particular location of the transmitter and receiver at the time of measurement. It is generally assumed that the channel is stationary in neglecting the time and location at which the impulse response is measured.

Discrete channel modeling is more convenient for channel simulation as each path component of the impulse response has random amplitude and phase, i.e. the complex envelope of the impulse response is represented by $[94,200]$

$$
h(t)=\sum_{r=0}^{R} \alpha_{r} \delta\left(t-t_{r}\right) \exp \left(-j \theta_{r}\right)
$$

The transmitted impulse $\delta(t)$ is thus received as the sum of $\mathrm{R}+1$ paths. As discussed in Chapter 2, measurements in a medium-size office building were used to develop an initial model [60] of the indoor channel where the path arrival times $t_{r}$ form a Poisson process with average arrival rate $\xi$ paths per second, the phases $\theta_{\mathrm{r}}$ are uniformly distributed and the amplitudes $\alpha_{\mathrm{r}}$ are Rayleigh distributed random variables whose variance is determined by evaluating $\phi_{\xi}(t)$, the multipath delay profile for a particular arrival rate $\xi$, at $t=t_{k}$. Hence, if one uses a discrete model to represent a given set of (measured) channel responses, the latter can be classified according to mean path arrival rate $\xi$. However, one can relate the discrete model to the continuous model by taking [94] the averagc received power over the entire set of responses for all delay values of interest and using the result as the multipath delay profile $\phi(t)$. In this case, regardless of the path arrivai rate, the continuous and discrete model multipath delay profiles are equivalent. 
Results of DFE modem analysis [94] without cochannel interference and using a discrete channel model have shown that, for the DFE performance to be insensitive to path arrival rate, the latter normalized by the rms delay spread $\sigma_{\mathrm{d}}$, should be at least 2 , i.e. $\xi \sigma_{d} \geq 2$ paths per rms delay spread interval. The bit-by-bit simulation described in this Chapter thus uses a simple discrete model where the $\left\{t_{r}\right\}$ are fixed and the $R+1$ complex gaussian channel tap coefficients are spaced by $\frac{\sigma_{d}}{2}$ i.e.

$$
h(t)=\sum_{r=0}^{R} \alpha_{r} \delta\left(t-\frac{r \sigma_{d}}{2}\right) \exp \left(-j \theta_{r}\right)
$$

where the $\theta_{\mathrm{r}}$ are uniformly distributed in $\{-T / 2, T / 2\}$ and the $\alpha_{\mathrm{r}}$ are Rayleigh distributed rando:n variables. Each of the $\alpha_{r}$ is equal to the magnitude of the $r^{\text {th }}$ channel tap coefficient, i.e.

$$
\alpha_{r}=\sqrt{x_{r}^{2}+y_{r}^{2}}
$$

These gaussian variates have identical variances equal to

$$
\begin{aligned}
& \left(\frac{1}{2}\right) \mathrm{E}\left[\alpha_{r}^{2}\right]=\mathrm{E}\left[x_{r}^{2}\right]=\mathrm{E}\left[y_{r}^{2}\right] \\
& =\left(\frac{1}{2}\right)\left(\frac{\sigma_{d}}{2}\right) \phi\left(\frac{\left(r+\frac{1}{2}\right) \sigma_{d}}{2}\right) \text { where } r=0,1, \ldots, R .
\end{aligned}
$$

The variances are normalized by the path spacing, equal to half the delay spread, in order to properly relate the discrete representation to the continuous model delay profile $\phi(t)$ which implicitly represents paths spaced by infinitesimal intervals, i.e. where the path arrival rate is infinite. As shown in Figure 4.1, equation 4.2 yields average path energies whose sum is a good approximation to the area (equal to 1) under $\phi(t)$. With $R+1=9$ paths and an rms delay spread equal to the symbol period $T$, the sum of normalized variances for the exponential profile $\phi(\tau)=\frac{1}{\sigma_{d}} e^{-\frac{\tau}{\sigma_{d}}}$ in Figure 4.1 was found to be $\sum_{r=0}^{8} E\left[\alpha_{r}^{2}\right]=0.9787$. As a tenth path only increased the sum of variances to $0.9830, R+1$ was set to 9 which yields impulse responses of duration equal to $4 \sigma_{d}$. $\mathrm{A}$ similar approximation error, i.e. under 2.5 percent, was found for other delay profiles (e.g. Gaussian) and various rms delay spreads $\sigma_{\mathrm{d}}$. In fact, the approximation error should not change with $\sigma_{d}$ as the latter determines path spacing. 
Exponential delay profile $p(t)$ (ms delayspread $=T$,

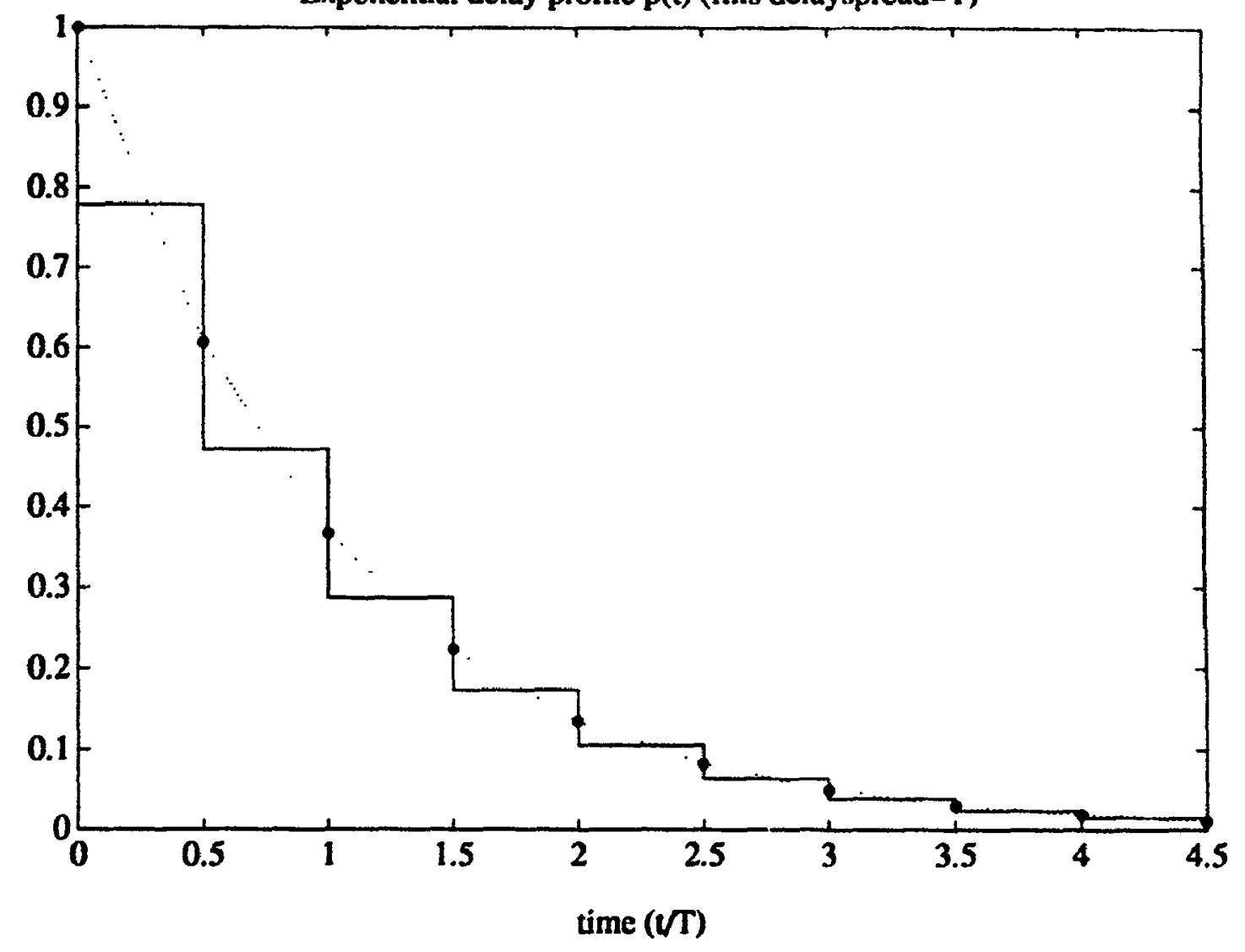

Figure 4.1 Exponential delay profile $\phi(t)$ (dotted line) with rms delay spread equal to one symbol period $T$. The bar chart (solid line) shows the path variance approximation (normalized to half the delay spread) as specified by equation 4.4 . 


\subsection{Static channel approximation}

The temporal variations in the Rayleigh fading channel characteristics can be simulated by passing the independent in-phase and quadrature Gaussian noise processes making up each tap coefficient $\alpha_{\mathrm{r}}$ (equation 4.3) of the dispersive channel, through a second-order Butterworth filter [201, 202]. The latter's $3 \mathrm{~dB}$ cut-off frequency controls the fade rate or the temporal rate of variation of the channel. However, as noted previously, the indoor channel is a very slowly varying medium; with average fade durations of up to 20 or even $\mathbf{4 0}$ seconds [75] with static ${ }^{\mathbf{4 3}}$ transmitter and receiver, the channel can be essentially static for up to $4 \times 10^{7}$ symbol intervals when considering data rates in the megabi/second range. Generating such a small fade rate (i.e. $2.5 \times 10^{-8} \mathrm{~Hz}$ with a simulated symbol rate normalized to one) yielded underflow problems as both the Butterworth filter cut-off frequency and the resulting output amplitude were very small. Even if one could overcome these numerical precision problems, the simulation would have to be run oyer an extremely high number of symbols in order to obtain a good average over the channel statistics, thus making the execution time unreasonable.

Hence, the average bit error rate for a given SINR is obtained by taking the average of error rates over a number of different sets of static channel realizations. In other words, for each portable-to-base-station-antenna path, different channel realizations are selected randomly from a Rayleigh distribution according to equations 4.3 and 4.4. After a brief interval reserved for equalizer training and AGC adjustment, i.e. automatic gain control ensuring a constant power level at the receiver inputs, the simulation is run with this static channel set over a sufficient number of symbols to count at least 100 errors in which the case the variance of the error rate for this given channel realization should be small. This yields an error rate for this particular channel set. A new set of channel realizations is then generated and another error rate is obtained. With a sufficiently large number of channel sets (and thus error rates), the average of all error rates provides a good estimate of the statistical mean error rate. Outage probabilities, which are of greater interest than average error rates for indoor wireless communications can also be obtained from these results. Note that this technique does not simulate the 43 Motion of the transmitter or receiver will decrease the average fade duration. 
equalizer adaptation process in normal mode of operation which is of lesser interest due to the quasi-static nature of the channel. In training mode, an adaptation (with the LMS algorithm) step size ${ }^{44}$ of 0.01 is used. In normal mode, it is set to 0.0001 but can be set to zero as the channel is static.

As shown in Figure 4.2 which displays

$$
\mathrm{E}\left[\left|\sum_{r=0}^{8}\left(x_{r}+j y_{r}\right)\right|^{2}\right]=\sum_{r=0}^{8}\left(\mathrm{E}\left[x_{r}^{2}\right]+\mathrm{E}\left[y_{r}^{2}\right]\right)
$$

versus the number of channel realizations, at least 100 realizations are needed to obtain a relatively constant mean magnitude squared output from a given portable-to-basestation antenna path. As expected from Figure 4.1, this constant is just slightly below 1 (i.e. - 0.98). With 100 channel sets, the distributions of each channel's output magnitude is then a close approximation to the corresponding Rayleigh distribution of the magnitude of the sum of the 9 channel tap coefficients (refer to Figure 4.3a) equal to

$$
\left|\sum_{r=0}^{8}\left(x_{r}+j y_{r}\right)\right|=\sqrt{\sum_{r=0}^{8}\left(x_{r}^{2}+y_{r}^{2}\right)}=\sqrt{\sum_{r=0}^{8} x_{r}^{2}+\sum_{r=0}^{8} y_{r}^{2}}
$$

and obtained with a very large (i.e. 100000) number of channel realizations. Hence, 100 channel sets should yield a reasonable approximation to the true statistical average error rate. However, in order to obtain reliable outage rates, the required number of channel sets is much larger, for $10^{-2}$ outage rates, one should use approximately 10000 channel sets, i.e. to count about 100 outages.

Unfortunately, running a bit-by-bit simulation of this relatively complex system over a statistically significant number of channel sets and number of symbols per channel set still constitutes an unreasonable task in terms of execution time on a SUN 3-60 TM45 workstation; e.g. 8 samples per symbol and 10000 channel sets with 100000

4t It should be noted that step size is meaningless unless the variance of the input samples is specified. A good "rule of thumb" is to set the step size within $\frac{01}{\text { Lelinput samplevariancel }}$ and L.(inpus cample variance) where $L$ denotes the number of taps. E.g., with $L=3$ taps, a satisfactory step size of 0.01 implies a input variance of up to $15 \mathrm{~dB}$.

45 SUN is a trademark of Sun Microsystems, Inc. 


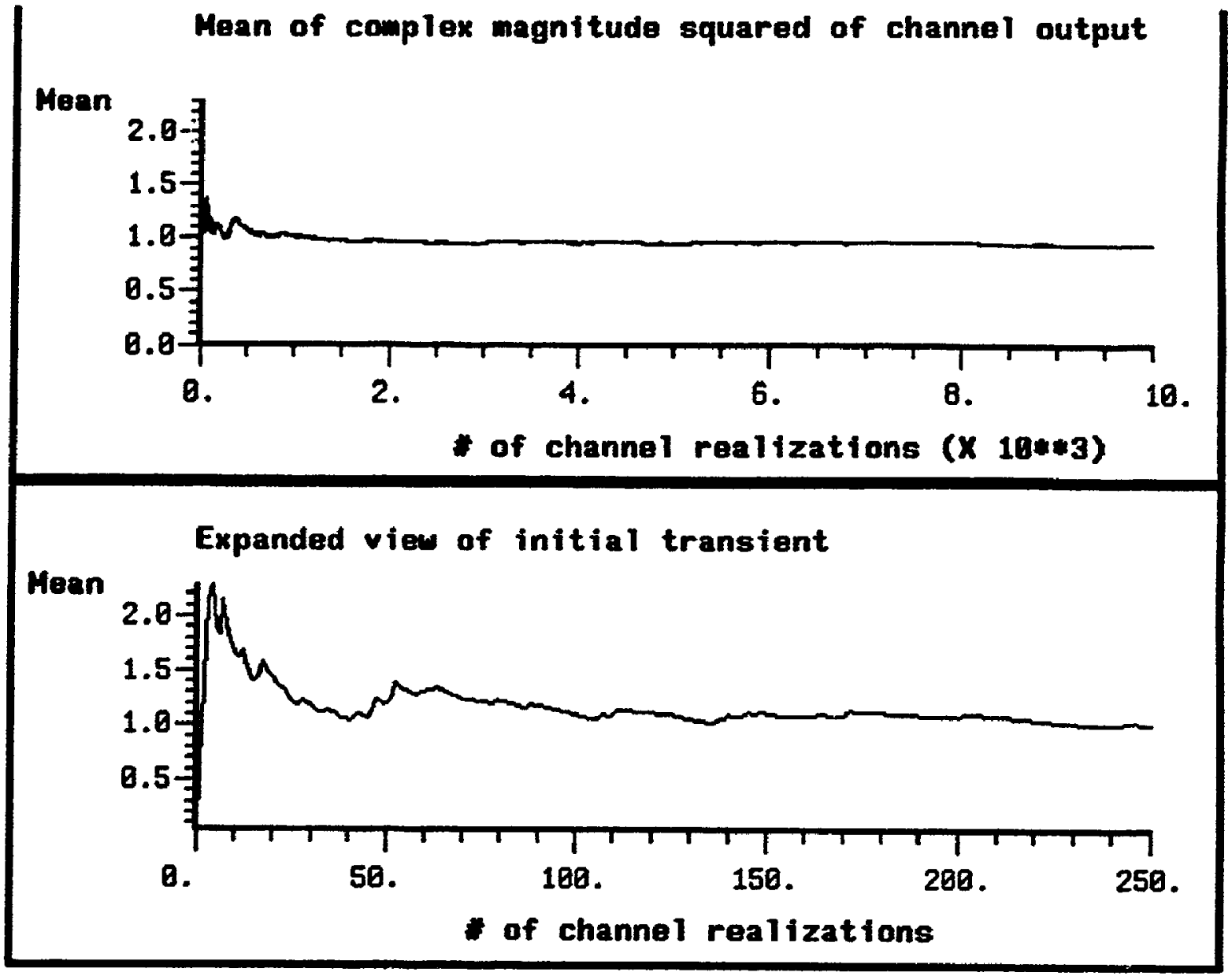

Figure 4.2 Mean of complex magnitude squared channel output (sum of complex magnitude squared of the 9 complex channel tap coefficients for a given portable to base-station-antenna path) versus number of channel realizations. 
symbols per channel set, which is sufficient to count ${ }^{-100}$ errors for BER's in the range of 0.001 , represents $10^{9}$ symbols or $8 \times 10^{9}$ samples! It was found that execution time constraints limitid the simulations to a few million symbols; hence, the BOSS simulation results shown in Chapter 4 were run with 20 channel sets and 100000 symbols per channel set, for a total simulation length of $2 \times 10^{6}$ symbols or $1.6 \times 10^{7}$ samples ${ }^{46}$. A histogram of a sample of 20 channel realizations, as is generated for every portable-to-base-station-antenna path, is shown in Figure 4.3b; the distribution has generally a Rayleigh shape but the small number of channel sets will substantially degrade the statistical accuracy of the simulation. It is assumed however that, by ensuring that all sets of $\mathbf{2 0}$ realizations on each portable-to-base-station-antenna path has this Rayleigh shape i.e. by avoiding a "clustered" set, reasonably significant results can be obtained. This technique would have to be modified in order to obtain outage rates; one could then use a sufficiently large number of channels (e.g. 10000) to count about 100 outages but only a few hundred symbols per channel set in order to maintain a total simulation length of a few million symbols. A criterion for "outage" over only a few hundred symbols would then have to be determined.

It is apparent that the statistical accuracy of the brute-force bit-by-bit simulation is subject to execution time constraints. The onus of a statistical approximation can be put on the number of channel sets when determining average error rates or on the number of symbols per channel set when determining outage rates. Semi-analytical error rate estimation techniques could also be considered to reduce execution time but only at the expense of introducing some assumptions.

46 It should be noted that four to five days of simulation time were required for $1.6 \times 10^{7}$ samples on the SUN 3-60 TM workstation available in 1989. At the time of writing this thesis, new and more sophisticated workstations such as the SPARC $2^{\mathrm{TM}}$ could reduce this execution time by approximately a factor of 15. 
a)

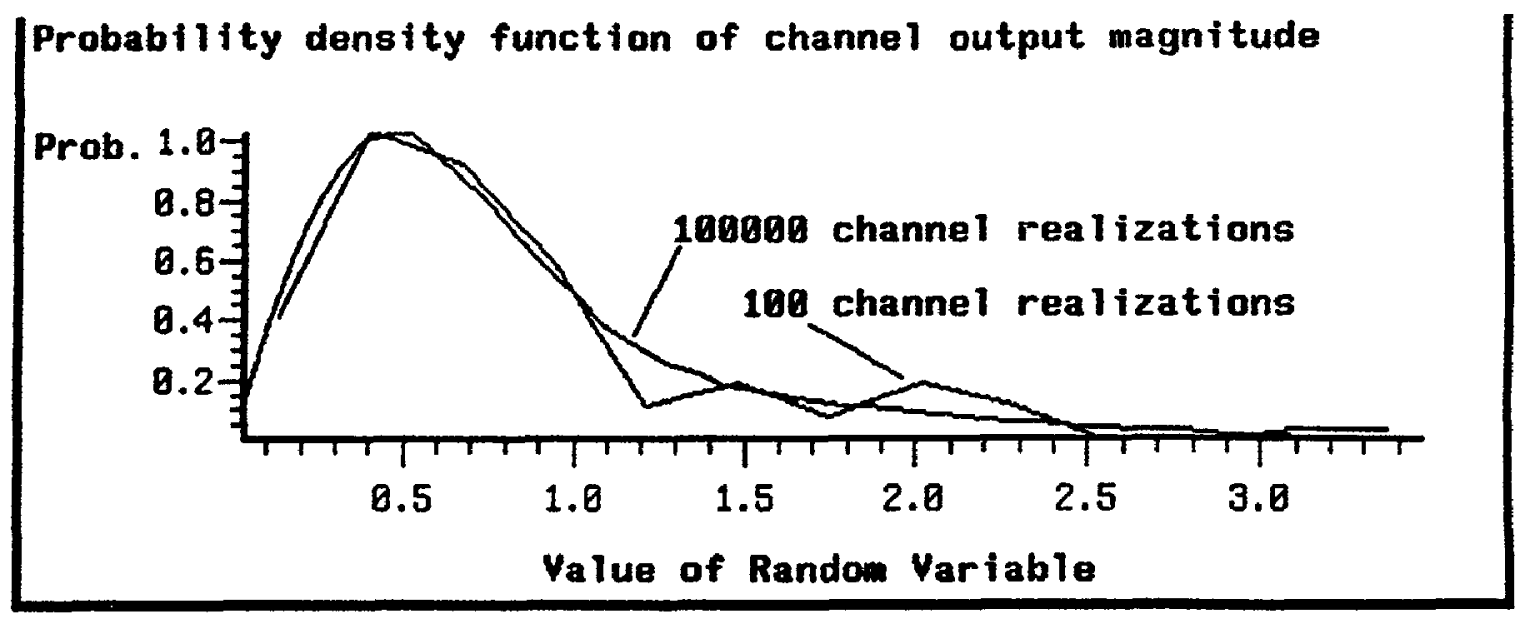

b)

Histogram of a set of 20 channel output complex magnitudes

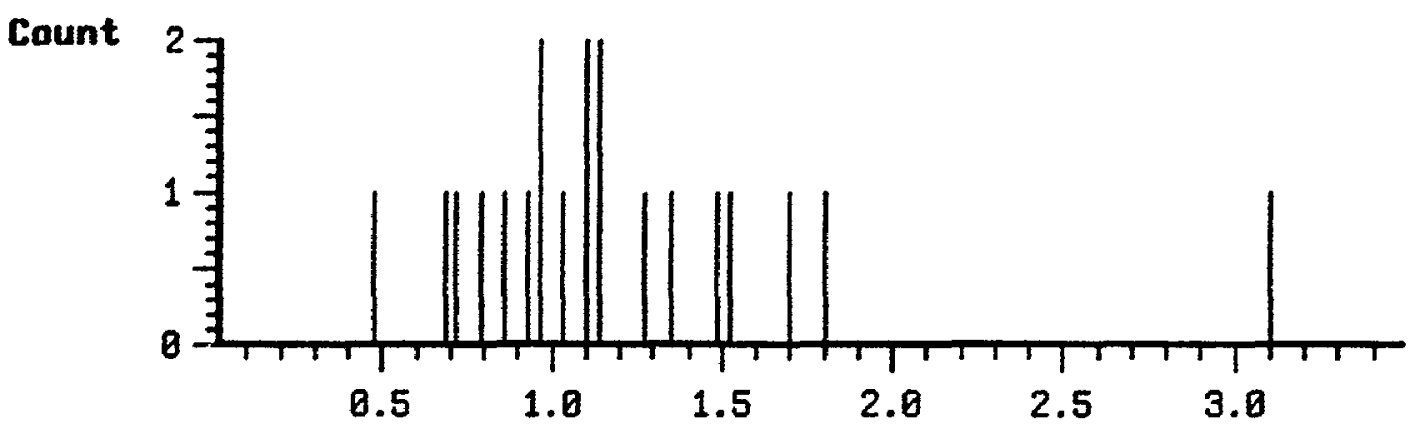

Valus of Bin Center

Figure 4.3 Distribution of channel output magnitude.

a) Probability density function for 100 and 10000 realizations.

b) Histogram for 20 realizations. 


\subsection{Results}

In this Section, the results of the bit-by-bit BOSS simulations are described and compared with the analytical predictions. All BOSS simulations in this work are based on a 9-tap discrete channel model with an exponential delay profile and a rms delay spread $\sigma_{d}=0.25 T$; a wide bandwidth time-rectangular pulse shape is used (no shaping) in order to maximize sampling phase sensitivity. The simulations employ the same code used in the analysis of Chapter 3, i.e. a rate $\frac{1}{2}$, constraint length 3 , free distance 5 convolutional code with code generators 5 and 7 . All the simulations with coding are performed without the use of interleaving. The cyclostationary nature of interference is initially assessed after which average BER and outage rate results are presented. The results of Figures 4.4 to 4.8 and of table 4.1 can be found on pages 114 to 119 .

\subsubsection{Cyclostationary nature of interference}

As mentioned in Section 3.7.2, an equalizer with the appropriate sampling phase can expluit the cyclostationary nature of interference to improve performance with respect to the case where the interference is stationary. In order to verify if the assumption of cyclostationary cochannel interference is realistic for the channel model being considered, a BOSS simulation was used to evaluate the average power of the sum of two independent in-phase BPSK signals each passed through independent frequencyselective Rayleigh fading channels. For each pair of static channel realizations, the power at different instants within a symbol interval was averaged over 1000 jymbols and then normalized to unity. The results for five different pairs of static channel realizations are shown in Figure 4.4. It is seen that the average power varies very differently in each case within a symbol interval and by as much as $6 \mathrm{~dB}$. It may then be concluded that the cochannel interference exhibits short-term cyclostationarity. Hence, performance gains could be achieved by properly exploiting this property of the various signals at the receiver input. If one then takes the average of a large number of such curves which is equivalent to averaging over the fading channel statistics, the resulting normalized average power is however found to be constant, i.e. equal to one ( 0 $\mathrm{dB}$ ), over the symbol interval. This indicates that in the long term, the interference must 
be considered as stationary. In addition, because the 2 signals pass through independent channel realizations with very different short-term delay profiles, the cyclostationary effect is not as pronounced and not so dependent on the relative timing phases of the interferers as in the DSL application [196, 197] where the signals go through similar channels. As the number of signais increases, the cyclostationary effect of $N$ in-phase signals will also eventually become negligible for our time-varying (fading) channel application. However, as one interferer usually dominates, the effect remains important.

\subsubsection{Average BER and outage performance}

As explained earlier, execution time constraints limited the bit-by-bit simulations to $2 \times 10^{6}$ symbols, which were split into 20 independent sets of channel realizations with $10^{5}$ symbols per set. As shown in the test case of Figure 4.5 where the dominant impairment is white gaussian noise (i.e. $\mathrm{N}=0)^{47}$, the simulation result agrees with the analytical prediction within $0.6 \mathrm{~dB}$ for $\frac{E_{b}}{N_{0}} \geq 2 d B$. In the latter range, the analytical assumption of correct feedback decisions thus seems to hold for the 20 realizations considered. However, for $\frac{E_{0}}{N_{0}}<2 d B$, this assumption certainly degrades and error propagation makes the analytical curve optimistic with respect to the simulation. The second crossover point at $8.3 \mathrm{~dB}$ is not very significant as the statistical accuracy of the simulation starts to degrade when the average BER falls below $10^{-3}$, i.e. with $10^{5}$ symbols per channel set, the average number of errors counted then falls below 100. Taking into account the analytical approximation of an exponential performa:ace bound for coherent detection, a simulation with $\mathbf{2 0}$ channel sets having approximately a Rayleigh distribution as shown in Figure $4.3 \mathrm{~b}$ thus appears, at this point, to provide a reasonable estimate of performance. In Figure 4.6a, one cochannel interferer $(N=1)$ accounting for half of the total noise-plus-interference power is now present; the behavior of the simulation curve is similar to the $\mathrm{N}=0$ case with the assumption of correct feedback decisions and the number of counted errors creating a discrepancy with the analysis for low and higher values of SINR respectively. It should also be noted that the analytical curves are dependent on the chosen value of the ISI-CCI gaussian symbol

47 ISI is also present but AWGN dominates for the range of $\frac{E_{0}}{N_{0}}$ considered. 
variance $\gamma^{2}$; as mentioned in Chapter 3, the latter could be "fine tuned" to provide a better match with simulation results. The simulation curves of Figures 4.5 and $4.6 \mathrm{a}$ are compared in Figure 4.6b. A $0.4 \mathrm{~dB}$ improvement, at an average BER of $10^{-3}$, is found when $\mathbf{N}$ goes from 0 to 1 ; this compares with $1.4 \mathrm{~dB}$ as predicted by the corresponding analytical curves (Figure 3.3). The statistical inaccuracy of the simulation coupled with the various analytical assumptions such as the exponential performance bound for coherent detection and the non-fading gaussian characterization of interference certainly affect this difference. In particular, the selected value of the gaussian variance $\gamma^{2}$ once again strongly influences the interference power in the analysis.

Average uncoded BER vs. $\frac{E_{0}}{N_{1}}$ curves are shown in Figures $4.7 \mathrm{a}$ for a fixed $S=10 d B$ and an equalizer sampling phase of $t_{0}=0.375 T$. A large discrepancy between analysis and simulation is observed for both the $M=2$ and $M=3$ cases. In addition, the rate $1 / 2$ coded QPSK system, used to maintain the same rate of information throughput with respect to the uncoded BPSK system, is found in Figure 4.8a to yield average $B E R$ performance inferior to the $M=3$ uncoded case and even asymptotically equivalent to the $\mathrm{M}=2$ uncoded case as $\frac{E_{8}}{N_{0}}$ increases. However, when the uncoded system's sampling phase is changed to $t_{0}=0.0 T$ as in Figure $4.7 \mathrm{~b}$, the simulation results now more closely match the analytical prediction and the coded $\mathbf{M}=2$ system outperforms both the uncoded $M=2$ system and the uncoded $M=3$ system. In the $M=3$ case, the small number of errors counted (i.e. on average $<100$ per channel set) pr. $x^{2}:$ bly contributes to the larger discrepancy at larger $\frac{E_{t}}{N_{10}}$. These large variations with sampling phase seem to :adicate that 20 chasinel sets are not sufficient to get a sritist ally valid result with respect to the sampling phase parameter. The latter's optimun value for this particular selection of 20 channel sets is thus quite different from $0.375 \mathrm{~T}$ which should be optimum over a large number of channel sets as predicted by the analysis of Chapter 3 . In fact, it was found that with $t_{0}=0.375 T$, two channel sets umiminated the average BER for both the uncoded and coded $M=2$ cases. Bit error rates for these two channel sets were larger than 0.1 even for $\frac{E_{0}}{N_{0}}=70 \mathrm{~dB}$. At this particular samuling ph... $\mathrm{P}_{\text {, }}$ the desired signal is most probably far weaker than the interferer on boch diversity n: $;$, shes. Table 4.1 a which displays the number of channel 
sets for which the average BER is larger than $10^{-3}$ when $t_{0}=0.375 T$, indicates that the addition of a third explicit space diversity branch enables the receiver to effectively cancel the cochannel and intersymbol interference as the error rate for those two channel sets falls substantially when AWGN is made insignificant, i.e. $\frac{E_{b}}{N_{i t}}=70 \mathrm{~dB}$. This also evident frum table 4.1c which shows the average BER when AWGN is negligible. However, when the sampling phase is $t_{0}=0.0 T$, tables $4.1 \mathrm{~b}$ and $4.1 \mathrm{c}$ show that good BER performance is obtained for all 20 channel sets even in the case of $M=2$ receiver antennas. Hence, if the optimum sampling phase for each channel set could be determined, e.g. by searching for the sampling phase which minimizes mean square error, or if the dependency on sampling phase is reduced by using a number of taps larger than 3, interference could be suppressed far more reliably with the uncoded $M=2$ receiver configuration. However, it remains to be seen how values of $\frac{S}{T}<10 d B$ or the addition of a second cochannel interferer would affect this conclusion.

In terms of an indoor portable radio environment where the channel characteristics change only very slowly, outage probability or the probability that the BER exceeds a certain threshold (e.g. $10^{-3}$ ) is generally of greater interest as a measure of performance than average BER. Of course with only 20 channel sets, the simulation results cannot be used to determine statistically valid outage rates. However tables $4.1 \mathrm{a}$ and $4.1 \mathrm{~b}$ clearly indicate that, when AWGN is significant, the $M=2$ coded system, although far more sensitive to sampling phase variations, could provide far lower outage rates than the uncoded $M=2$ and $M=3$ configurations even if the corresponding average $B E R$ is equal or larger. When AWGN is negligible, i.e. for $\frac{E_{b}}{N_{0}}=70 \mathrm{~dB}$, such a conclusion cannot necessarily be made as, for the case of $\frac{S}{T}=10 d B$ and given the proper sampling phase, both the uncoded and coded configurations yield very small average error rates and most probably small outage rates. The coded system's outage rate advantage can be explained by noting that the coded results exhibit a greater on-off behavior than the uncoded results for all values of $\frac{E_{b}}{N_{0}}$ considered, i.e. the coded BER for each channel set was either larger than 0.1 or very small. It thus would be informative to determine if the coded system's advantage still holds for $\frac{S}{I}<10 \mathrm{~dB}$ where the analysis of Chapter 3 predicts that coded performance could suddenly degrade. 


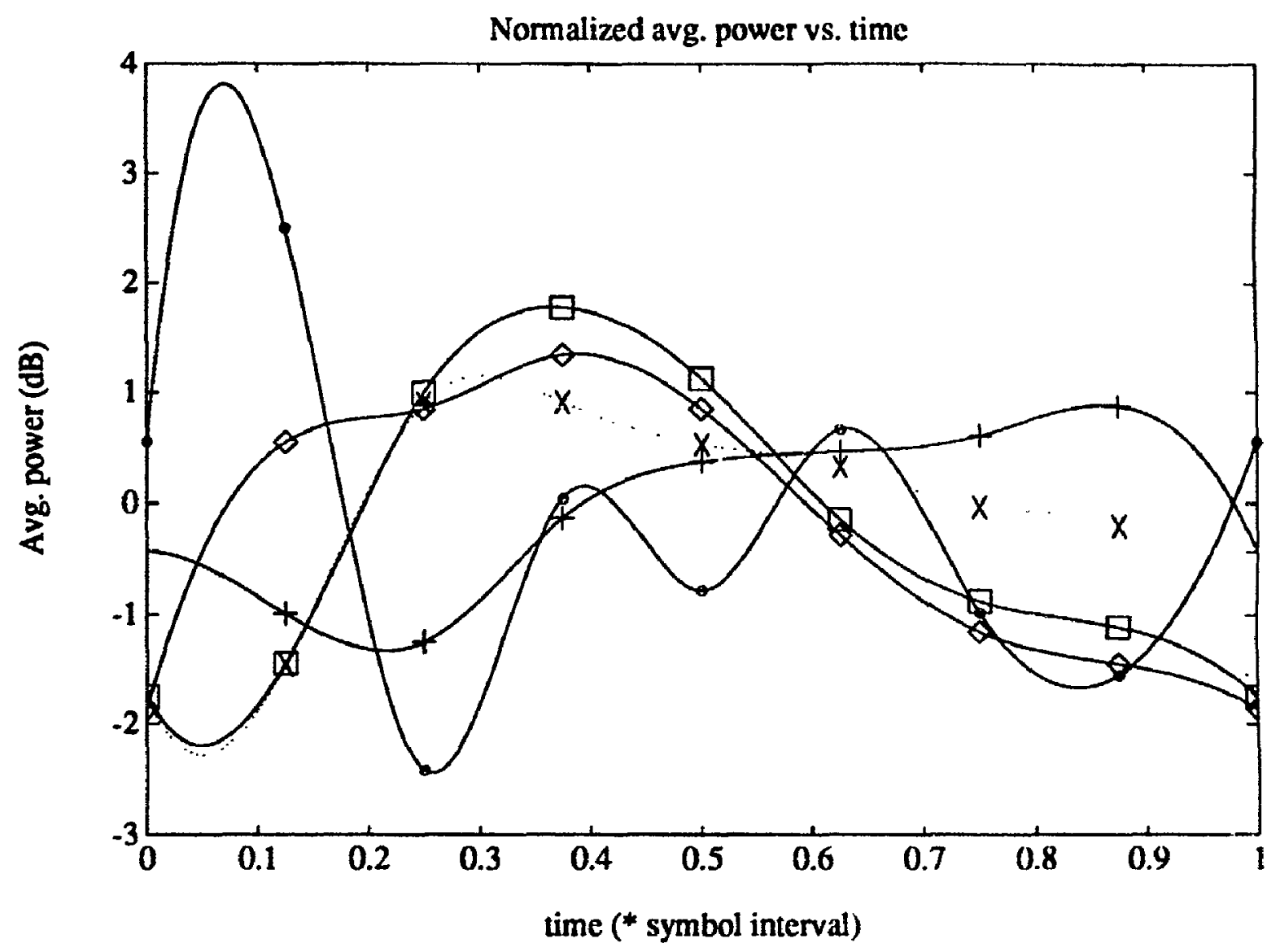

Figure 4.4 Average power (normalized to unity) of the sum of two independent in-phase BPSK signals passed through five different pairs of independent static dispersive Rayleigh fading channel realizations. 
Bit error rate vs. Eb/NO

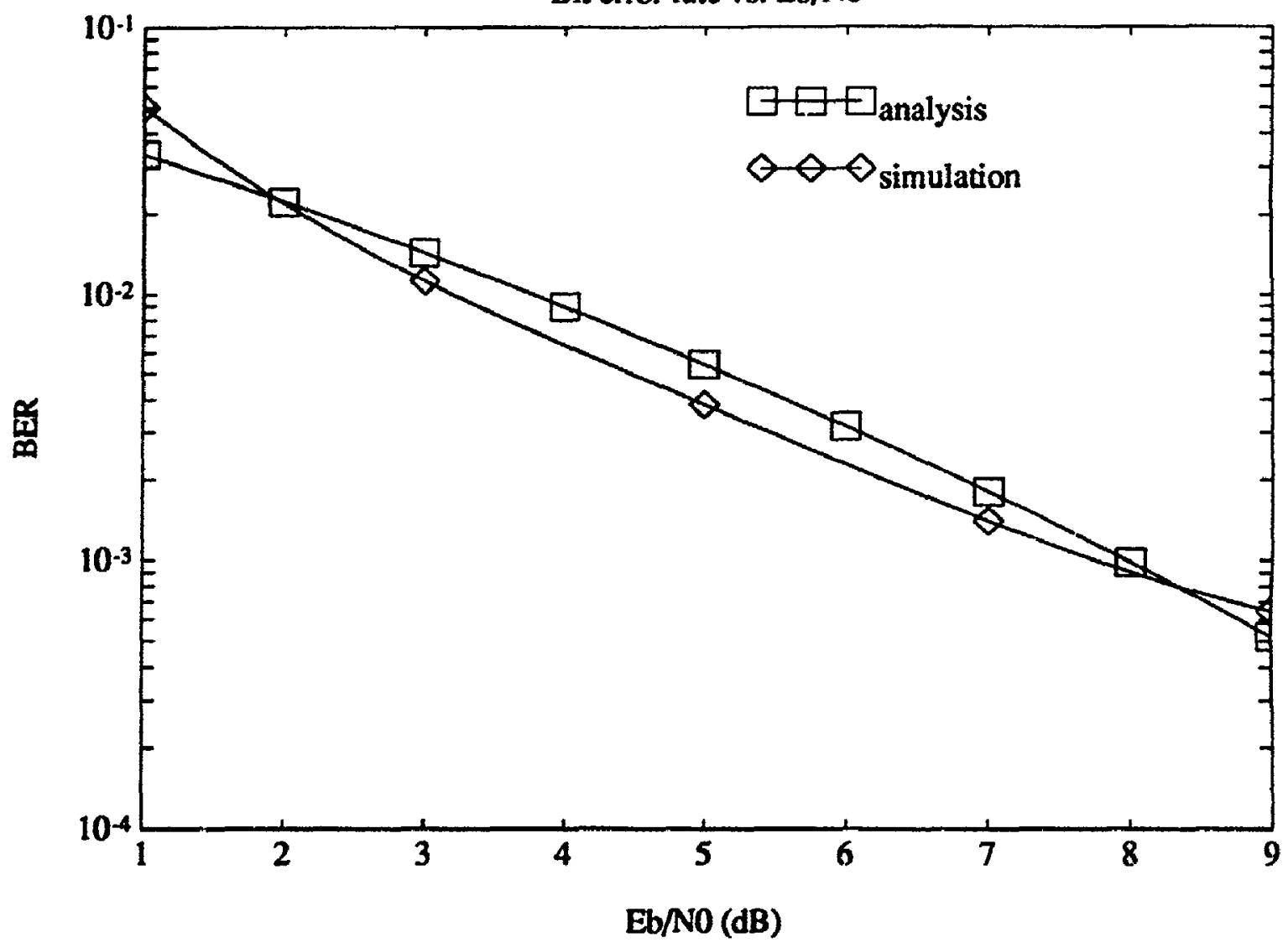

Figure 4.5 BPSK average BER vs. Eb/NO. $M=2, N=0, t_{0}=0.0 T$. 

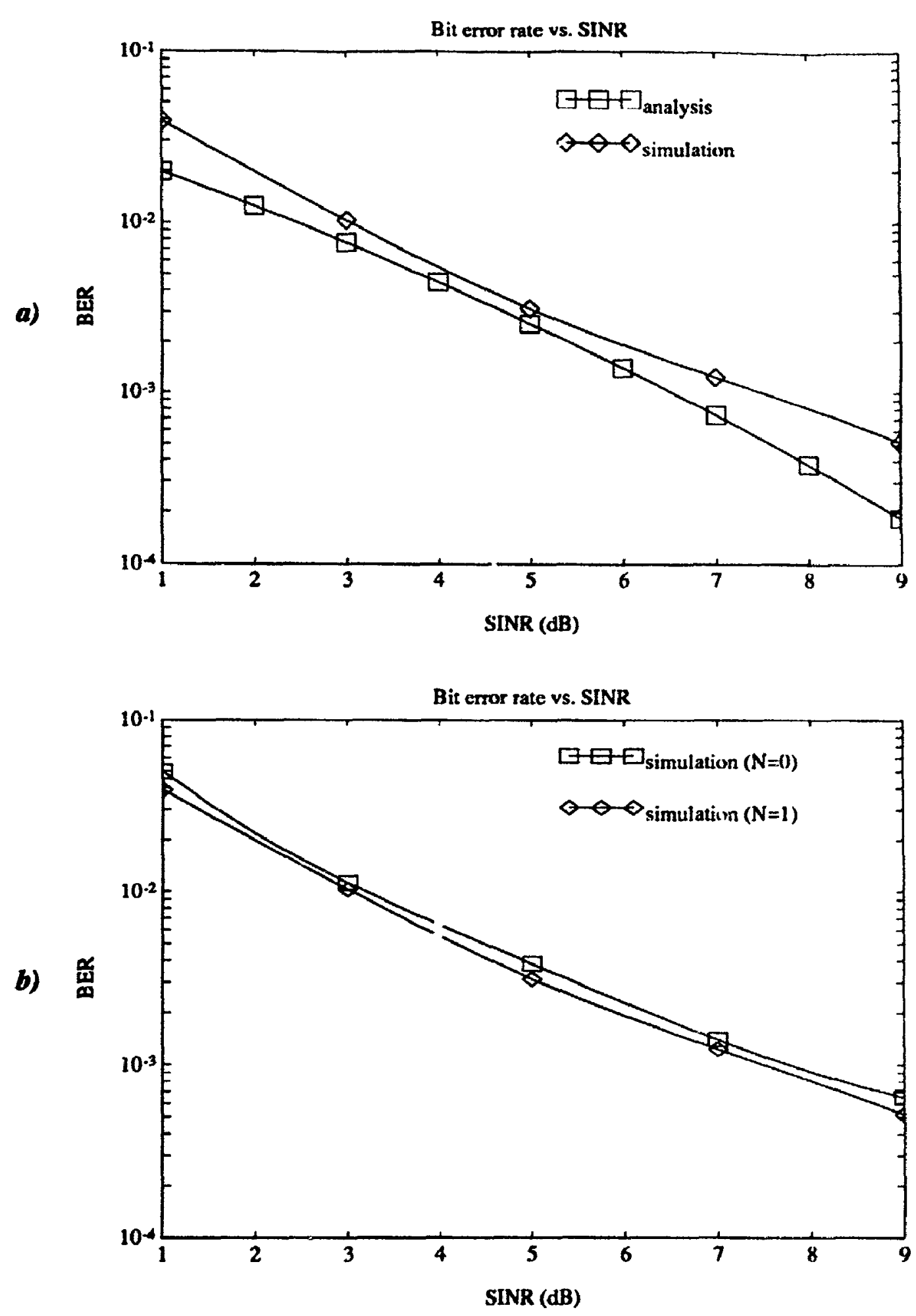

Figure 4.6 BPSK average BER vs. SINR for $10=0.0 T$.

a) analysis vs. simulation for $M=2, N=1, E s 1 / N 0=1, t 1=0.125 T$.

b) simulation results for $\mathrm{N}=0$ and $\mathrm{N}=1$ ( $\mathrm{Es} 1 / \mathrm{N} 0=1, t 1=0.125 \mathrm{~T})$. 

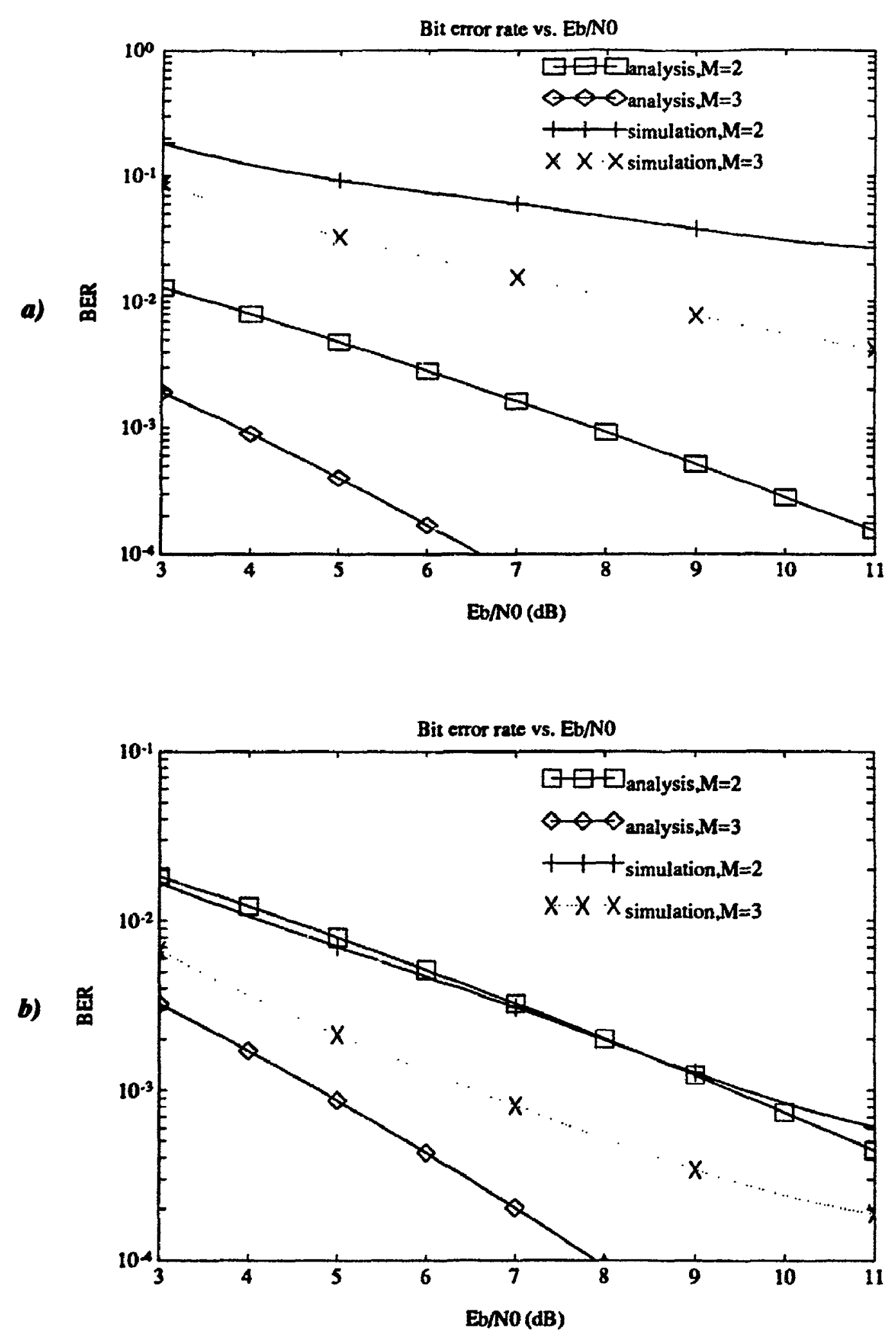

Figure 4.7 Analysis vs. simulation for BPSK average BER vs. Eb/No. $N=1, S / I=10 a B, t 1=0.0 \mathrm{~T} . \quad$ a) $t_{0}=0.375 T$. b) $t_{0}=0.0 \mathrm{~T}$. 

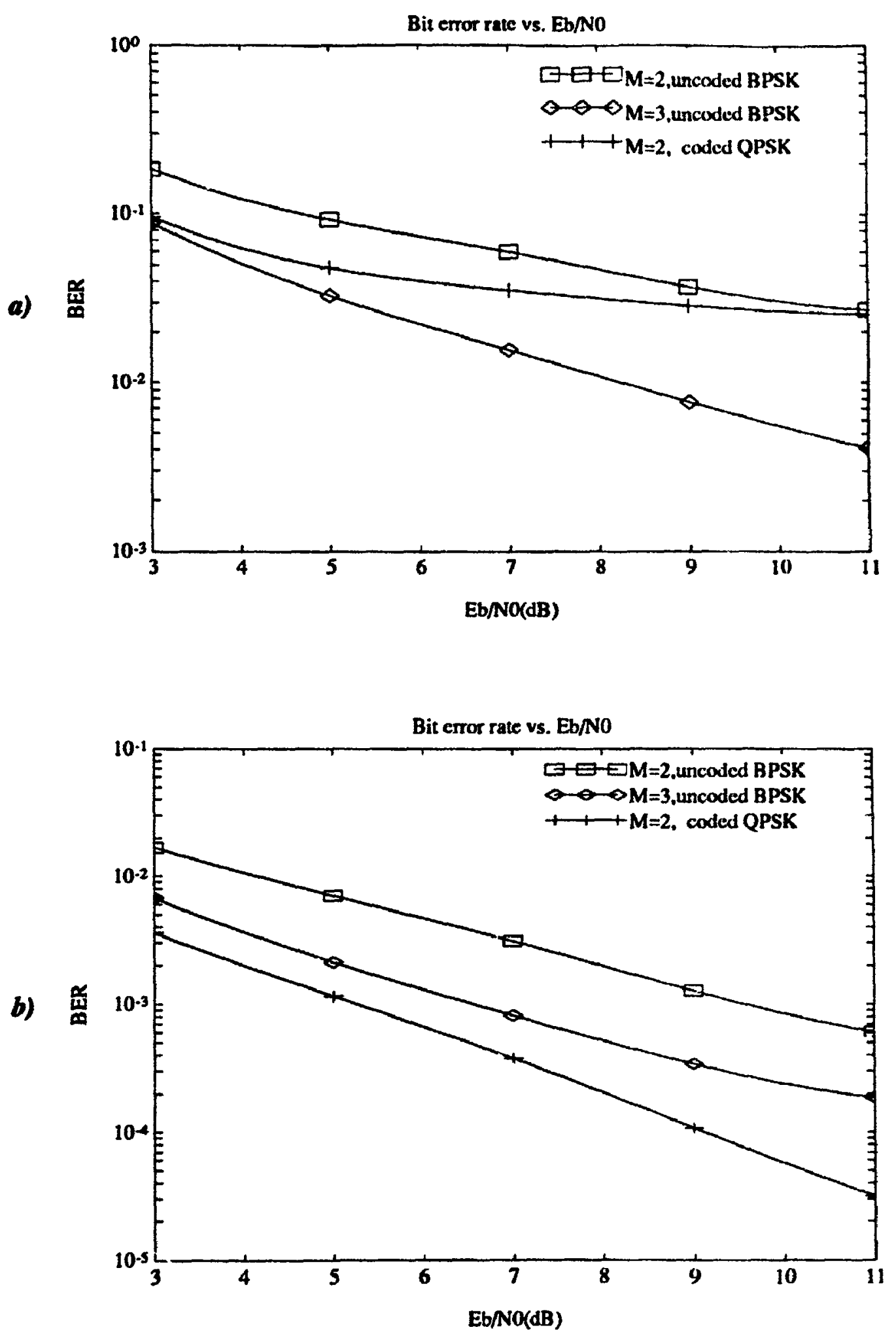

Figure 4.8 Simulation results for average BER vs. Eb/No, $\mathrm{N}=1, \mathrm{~S} / \mathrm{I}=10 \mathrm{~dB}, \mathrm{t} 1=0.0 \mathrm{~T} . \quad$ a) $\mathrm{t}_{0}=0.375 \mathrm{~T} . \quad$ b) $\mathrm{t}_{0}=0.0 \mathrm{~T}$. 
a)

\begin{tabular}{|c|c|c|c|c|}
\hline Eb/NO & $\begin{array}{c}M=2, \text { uncoded } \\
\text { BPSK }\end{array}$ & $\begin{array}{c}M=2, \text { coded } \\
\text { BPSK }\end{array}$ & $\begin{array}{c}M=2, \text { coded } \\
\text { QPSK }\end{array}$ & $\begin{array}{c}M=3, \text { uncoded } \\
\text { BPSK }\end{array}$ \\
\hline $5 \mathrm{~dB}$ & 19 & 8 & 12 & 19 \\
\hline $7 \mathrm{~dB}$ & 16 & 7 & 7 & 16 \\
\hline $9 \mathrm{~dB}$ & 16 & 6 & 4 & 15 \\
\hline $11 \mathrm{~dB}$ & 12 & 6 & 3 & 11 \\
\hline $70 \mathrm{~dB}$ & 2 & 2 & 2 & 0 \\
\hline
\end{tabular}

b)

\begin{tabular}{|c|c|c|c|c|}
\hline $\mathbf{E b} / \mathbf{N} 0$ & $\begin{array}{c}\text { M=2, uncoded } \\
\text { BPSK }\end{array}$ & $\begin{array}{c}\text { M=2, coded } \\
\text { BPSK }\end{array}$ & $\begin{array}{c}\text { M=2, coded } \\
\text { QPSK }\end{array}$ & $\begin{array}{c}\text { M=3, uncoded } \\
\text { BPSK }\end{array}$ \\
\hline $5 \mathbf{d B}$ & 17 & 4 & 4 & 17 \\
\hline $7 \mathrm{~dB}$ & 12 & 1 & 2 & 10 \\
\hline $9 \mathrm{~dB}$ & 7 & 1 & 1 & 6 \\
\hline $11 \mathrm{~dB}$ & 4 & 1 & 0 & 3 \\
\hline $70 \mathrm{~dB}$ & 0 & 0 & 0 & 0 \\
\hline
\end{tabular}

c)

\begin{tabular}{|c|c|c|c|c|}
\hline $\begin{array}{c}\text { sampling } \\
\text { phase }\end{array}$ & $\begin{array}{c}\text { M=2, uncoded } \\
\text { BPSK }\end{array}$ & $\begin{array}{c}\text { M=2, coded } \\
\text { BPSK }\end{array}$ & $\begin{array}{c}\text { M=2, coded } \\
\text { QPSK }\end{array}$ & $\begin{array}{c}\text { M=3, uncoded } \\
\text { BPSK }\end{array}$ \\
\hline $0.375 T$ & $2.46 \mathrm{E}-2$ & $4.02 \mathrm{E}-2$ & $2.55 \mathrm{E}-2$ & $1.40 \mathrm{E}-5$ \\
\hline $0.0 \mathrm{~T}$ & $1.85 \mathrm{E}-5$ & 0 & 0 & $8.2 \mathrm{E}-6$ \\
\hline
\end{tabular}

Table 4.1 Simulation results for $N=1, S / I=10 \mathrm{~dB}, t_{1}=0.0 \mathrm{~T}$

a) Number of channel sets (out of 20) for which average BER $\geq 10^{-3}$ vs. Eb/NO. $t_{0}=0.375 T$.

b) Number of channel sets (out of 20) for which average BER $\geq 10^{-3}$ vs. Eb/NO. $t_{0}=0.0 T$.

c) Average BER for 2 different sampling phases at $\mathrm{Eb} / \mathrm{N} 0=70 \mathrm{~dB}$. 


\subsection{Summary}

Following a simulation used to confirm the cyclostationary nature of the interference at the receiver antennas, bit-by-bit simulation results, with $L=3$ forward filter taps, $N=1$ cochannel interferer with $\frac{S}{T}=10 \mathrm{~dB}$ and a channel with an exponential delay profile and a rms delay spread $\sigma_{d}=0.25 T$, showed that the coded, non-interleaved system was more dependent on sampling phase than the uncoded system. It would thus be useful to use more than three taps or to continuously update the equalizer sampling phase to minimize mean-squared error. Given the proper sampling phase or more taps which alleviates the effect of sampling phase, it appears that using convolutional coding with soft-decision Viterbi decoding and no interleaving, could still be a valid alternative, in terms of outage rate, to increasing the space diversity order from $\mathbf{M = 2}$ to $M=3$. As mentioned in Chapter 1 , the viability of coding, whose implementation can be made very compact witii VLSI technology, becomes important when constraints on portable size limit the number of antennas. The on-off behavior of the coded, noninterleaved results also lead to an outage improvement for a suboptimal sampling phase even if the corresponding average BER was much worse than for the uncoded cases. It remains to be seen however, if these conclusions will also hold in the presence of more than one interferer or for lower values of $\frac{S}{T}$ where analysis predicts that coded system performance will suddenly degrade. This will be examined in the following Chapter. 


\section{Chapter 5 \\ SEMI-ANALYTICAL PERFORMANCE EVALUATION}

\subsection{Introduction}

This Chapter describes a semi-analytical approach involving the calculation, via the method of moments, of optimum baseband combiner performance over a static channel and Monte Carlo averaging over the fading distribution. In addition to providing results for both ideal interleaving and no interleaving, such a technique eliminates the nonfading gaussian noise characterization of interference inherent in the purely analytical approach of Chapter 3 while producing statistically significant results much faster than the simulation described in Chapter 4. The technique is initially described after which the remaining analytical assumptions are summarized. A large number of results are then presented and discussed.

\subsection{Description of technique}

The analytical performance bounds obtained in Chapter 3 were based on the assumption that the intersymbol and cochannel interference were normally (gaussian) distributed. In practice, this is not very realistic particularly when one interferer dominates as discussed in Chapter 2. In addition, approximating the short-term power of the dispersed interfering symbols by their long-term means over the channel statistics does not account for variations in short-term delay profiles which could degrade the accuracy of outage probabilities obtained via such a technique. For a particular fading channel realization, the optimum baseband combiner probability of bit error can be 
written as

$$
\begin{aligned}
P_{b}= & \frac{1}{2} \operatorname{Pr}\left(\operatorname{Re}(z)<0 \mid a_{00}=+1\right)+ \\
& \frac{1}{2} \operatorname{Pr}\left(\operatorname{Re}(z)>0 \mid a_{00}=-1\right)
\end{aligned}
$$

which becomes by symmetry

$$
P_{b}=\operatorname{Pr}\left(\operatorname{Re}(z)<0 \mid a_{00}=+1\right)
$$

and where, as shown in Chapter 3, the decision variable is

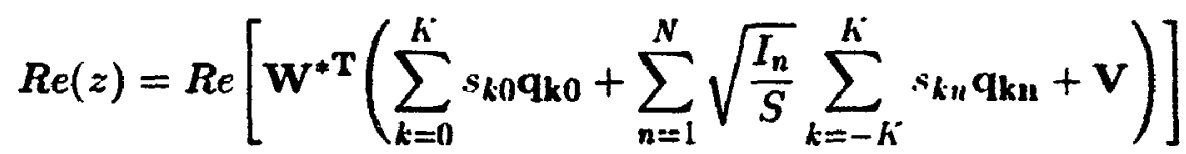

with $s_{k n}=a_{k n}+j b_{k n}$. Calculating $P_{b}$ exactly is most dificicult for all possible combinations of $s_{k n}= \pm_{-1}^{+} j, \quad(k=-K, \ldots K$ and $n=0, \ldots . N)$ must be taken into account except for $\operatorname{Re}\left(s_{\mathrm{m}}\right)=a_{00}$. This implies that computational complexity will grow exponentially, for BPSK, as a function of $2^{2 \kappa(N+1)}$ which precludes using such an approach even for small $K$ and $N^{48}$.

Two approaches have been considered to overcome this difficulty. The first involves setting bounds on the error probability as done by Glave and Rosenbaum [203]. Tighter bounds were also obtained by Yao and Biglieri [204] but their computation is extremely complicated. In addition, making these bounds good approximations to $P_{b}$ can require lengthy calculations. Another approach for computing $P_{b}$ is attributable to Benedetto et al. [205, 206] who noticed that the problem of error probability evaluation in the presence of intersymbol and cochannel interference could be solved by using a Gaussian-quadrature (GQR) algorithm [207]. It involves computing definite integrals of the form $\int_{x_{1}}^{x_{2}} f(x) w(x) d x$ where the integrand has been factored into a continuous term $f(x)$ and a non-negative term $w(x)$ such as a probability density function of a random variable $X$. Golub and Welsch [207] have develeped an algorithm where only a finite number of the moments of $X$ are required to find the desired integration rule without complete knowledge of $w(x)$, thus the name method of moments. More efficient

4 Consider that for $K=3$ and $N=1,2^{2 K(N+1)}=4096$. 
variations of the Golub and Welsch technique have also been developed by Gautschi [208] and by Meyers [209]. However, the GQR technique is still fairly long and intricate $^{49}$ and complexity still grows exponentially with $\mathrm{N}$ and $\mathrm{K}$.

Recently, Helstrom [59] proposed a simpler technique, in terms of implementation, where the probability of error is calculated by numerical quadrature of a complex Laplace transform inversion integral. The latter's integrand contains the momentgenerating functions ${ }^{50}$ (mgf) of the interference variate and the noise. Given $y=\operatorname{Re}(z)$ and $g(s)$ the mgf of $y$, the probability density function of $y$ is

$$
p(y)=\frac{1}{2 \pi j} \int_{c-j \infty}^{c+j \infty} g(s) \exp (s y) d s
$$

with integration along a contour parallel to the imaginary s-axis. The bit error probability is then simply

$$
\begin{aligned}
P_{b} & =\int_{-\infty}^{0} p(y) d y=\frac{1}{2 \pi j} \int_{c-j \infty}^{c+j \infty} g(s) \int_{-\infty}^{0} \exp (s y) d y d s \\
& =\frac{1}{2 \pi j} \int_{c-j \infty}^{c+j \infty} s^{-1} g(s) d s
\end{aligned}
$$

By taking a vertical contour of integration through the saddlepoint of the integrand $s^{-1} g(s)$, Helstrom [59] has shown that the number of steps of numerical integration required for a certain accuracy is kept to a minimum and that the computational complexity increases only linearly with $\mathbf{N}$ and $\mathrm{K}$. The procedure requires only two simple numerical analysis techniques namely Newton's method in locating the saddlepoint and the trapezoidal rule for numerical integration. $A s P_{b}$ is determined via the momentgenerating function and without explicit knowledge of $p(y)$, this technique is essentially ancther version of the aforementioned method of moments.

Helstrom's paper [59] is based on a binary symmetric channel with ISI, CCI and AWGN. This technique is now extended to find the bit error probability for a given

49 The procedure typically entails finding the eigenvalues and eigenvectors of a tridiagonal matrix.

so This is simply the Laplace transform version of the characteristic function defined as the Fourier transform of the probability density function of the variate. 
channel set realization of both the uncoded and coded optimum baseband combiner systems with BPSK and QPSK modulation.

\subsubsection{Uncoded BER}

By expanding equation 5.3 and defining ${ }^{51} A_{k n} \triangleq \sqrt{\frac{I_{n}}{S}} \operatorname{Rc}\left(\mathbf{W}^{* T} \mathbf{q}_{\mathbf{k n}}\right)$ and $B_{k, n} \triangleq$ $\sqrt{\frac{I_{\mathbf{m}}}{S}} \operatorname{Im}\left(\mathbf{W}^{* \mathbf{T}} \mathbf{q}_{\mathbf{k n}}\right)$, the decision variable for a particular bit can be expressed as

$$
\begin{aligned}
& y=\operatorname{Re}(z) \\
& =a_{00} A_{00} \quad \text { (desired symbol) } \\
& +\sum_{k=1}^{K} a_{k 0} A_{k 0} \\
& +\sum_{n=1}^{N} \sum_{k=-K}^{K}\left[a_{k n} A_{k n}-b_{k n} B_{k n}\right] \\
& +\operatorname{Re}\left(\mathbf{W}^{* \mathbf{T}} \mathbf{V}\right) \\
& =a_{00} A_{00}+\eta+\xi
\end{aligned}
$$$$
-\sum_{k=0}^{K} b_{k 00} B_{k 00} \quad \text { (crosis - rail interf.) }
$$

where $\eta$ is the sum of ISI, CCI and CRI (cross-rail interference) ${ }^{52}$ and $\xi$ is the additive white gaussian noise. Setting $a_{00}=1$ in order to compute $P_{b}$ from equation 5.2, and because the interference and the noise are statistically independent, the momentgenerating function (mgf) of the decision variable is

$$
g(s)=\mathrm{E}[\exp (-\mathrm{sy})]=g_{\eta}(s) g_{\xi}(s) \exp \left(-A_{r(10} . s\right)
$$

5t For the case of $n=0$ (desired signal), $\sqrt{\frac{L_{n}}{S}}=1$.

52 Note that for BPSK, all $b_{k n}=0$ which eliminates the cross-rail interference. 
With all $a_{k n}$ and $b_{k n}$ being independent, the mgf of the interference can be written as

$$
\begin{aligned}
& g_{\eta}(s)=\left[\exp \left(-s\left(\sum_{k=1}^{K} a_{k 0} A_{k 0}-\sum_{k=0}^{K} b_{k \cdot 0} B_{k 0}+\sum_{n=1}^{N} \sum_{k=-K}^{K}\left(a_{k n} A_{k n}-b_{k n} B_{k n}\right)\right)\right)\right] \\
& =\left(\prod_{k=1}^{K} \frac{1}{2}\left[\exp \left(A_{k \cdot 0} s\right)+\exp \left(-A_{k 0} s\right)\right]\right) \cdot\left(\prod_{k=0}^{K} \frac{1}{2}\left[\exp \left(B_{k 0} s\right)+\exp \left(-B_{k n} s\right)\right]\right) \\
& \cdot\left(\prod_{n=1}^{N} \prod_{k=-K}^{K}\left(\frac{1}{2}\left[\exp \left(A_{k n} s\right)+\exp \left(-A_{k n} s\right)\right]\right)\left(\frac{1}{2}\left[\exp \left(B_{k n} s\right)+\exp \left(-B_{k n} s\right)\right]\right)\right) \\
& =\prod_{k=1}^{K} \cosh \left(A_{k 0} s\right) \prod_{k=0}^{K} \cosh \left(B_{k 0} s\right) \prod_{n=1}^{N} \prod_{k=-K}^{K}\left[\cosh \left(A_{k n} s\right) \cosh \left(B_{k n} s\right)\right]
\end{aligned}
$$

Note that with the AWGN component $\xi$ written as

$$
\xi=\operatorname{Re}\left(\mathbf{W}^{* \mathbf{T}} \mathbf{V}\right)=\sum_{i=1}^{M L}\left(\operatorname{Re}\left(w_{i}\right) n_{c i}-\operatorname{Im}\left(w_{i}\right) n_{s i}\right)
$$

where the $n_{c i}$ and $n_{s i}$ are independent zero mean gaussian variates with variance $\frac{N_{n}}{2}$, the variance of $\xi$ is seen to be $\mathrm{b}^{53}$

$$
\begin{aligned}
\operatorname{var}\left[\operatorname{Re}\left(\mathbf{W}^{* \mathbf{T}} \mathbf{V}\right)\right]= & \frac{N_{\mathbf{6}}}{2}\left[\operatorname{sum}\left(\operatorname{Re}^{2}(\mathbf{W})\right)+\operatorname{sum}\left(\operatorname{Im}^{2}(\mathbf{W})\right)\right] \\
& =\frac{N_{0}}{2} \operatorname{sum}\left(|\mathbf{W}|^{2}\right)
\end{aligned}
$$

Since the $\mathrm{mgf}$ of zero-mean AWGN with variance $\sigma^{2}$ is [71] $\exp \left(-\frac{\sigma^{2} s 2}{2}\right)$, the $\mathrm{mgf}$ of $\xi$ becomes.

$$
g_{\xi}(s)=\exp \left(\frac{N_{0} s^{2}}{4} \operatorname{sum}\left(|\mathbf{W}|^{2}\right)\right)
$$

As in Helstrom [59], the integral of equation 5.5 will now be evaluated by taking a straight vertical path through the single ${ }^{54}$ positive saddlepoint (i.e. where the integrand 53 As indicated in the list of mathematical symbols and notation, $\operatorname{sum}(\mathrm{X})$ simply refers to the sum of the elements of the vector $\mathrm{X}$.

54 This results from the convexity of the moment-generating function [59]. 
is minimum) $c=s_{0}$ on the positive $R e(s)$ axis. Since the natural logarithm of the integrand is also a monotonically increasing function on the positive $\operatorname{Re}(s)$ axis, the saddlepoint $s_{0}$ may be found by solving for the positive root of

$$
\frac{d \phi(s)}{d s}=\phi^{\prime}(s)=0
$$

where

$$
\phi(s)=\ln \left(s^{-1} g(s)\right)
$$

As $\phi^{\prime}(s)$ is a fairly complex expression, the saddlepoint can be determined via Newton's method where an initial value of $s_{0}$ is replaced by [59]

$$
s_{0} \leftarrow s_{0}-\frac{\left.\phi^{\prime}(s)\right|_{s=s_{11}}}{\left.\phi^{\prime \prime}(s)\right|_{s=s_{11}}}
$$

In our case, equations 5.7, 5.8 and 5.11 lead to

$$
\begin{aligned}
\phi(s)= & \sum_{k=1}^{K} \ln \left[\cosh \left(A_{k \cdot(\cdot)}\right)\right]+\sum_{k=0}^{K} \ln \left[\cosh \left(B_{k \cdot 0} \cdot\right)\right] \\
& +\sum_{n=1}^{N} \sum_{k=-K}^{K}\left(\ln \left[\cosh \left(A_{k n} s\right)\right]+\ln \left[\cosh \left(B_{k \cdot n^{*}}\right)\right]\right) \\
& +\frac{N_{0}}{4} s^{2} \operatorname{sum}\left(|\mathbf{W}|^{2}\right)-A_{00} s-\ln (s)
\end{aligned}
$$

and

$$
\begin{aligned}
\phi^{\prime}(s)= & \sum_{k=1}^{K} A_{k 0} \tanh \left(A_{k 0} s\right)+\sum_{k=0}^{K} B_{k 0} \tanh \left(B_{k(1) s}\right) \\
& +\sum_{n=1}^{N} \sum_{k=-k}^{K}\left(A_{k n} \tanh \left(A_{k n s}\right)+B_{k+n} \tanh \left(B_{k n} s\right)\right) \\
& +\frac{N_{0} s}{2} \operatorname{sum}\left(|\mathbf{W}|^{2}\right)-A_{00}-s^{-1}
\end{aligned}
$$

and

$$
\begin{aligned}
\phi^{\prime \prime}(s)= & \sum_{k=1}^{K} A_{k 0}^{2} \operatorname{sech}^{2}\left(A_{k 0} s\right)+\sum_{k=0}^{K} B_{k 0}^{2} \operatorname{sech}^{2}\left(B_{k(1)} s\right) \\
& +\sum_{n=1}^{N} \sum_{k=-K}^{K}\left(A_{k n}^{2} \operatorname{sech}^{2}\left(A_{k n} s\right)+B_{k n}^{2} \operatorname{sech}^{2}\left(B_{k n} s\right)\right) \\
& +\frac{N_{0}}{2} \operatorname{sum}\left(|\mathbf{W}|^{2}\right)+s^{-2}
\end{aligned}
$$


A practical starting point for the saddlepoint search is obtained by replacing the interference terms by Gaussian noise of equal variance [59] yielding a total variance of

$$
\begin{aligned}
\sigma_{0}^{2}= & \frac{N_{0}}{2} \operatorname{sum}\left(|\mathbf{W}|^{2}\right)+B_{00}^{2}+\sum_{k=1}^{K}\left(A_{k 0}^{2}+B_{k 0}^{2}\right) \\
& +\sum_{n=1}^{N} \sum_{k=-K}^{K}\left(A_{k n}^{2}+B_{k n}^{2}\right) \\
= & \frac{N_{0}}{2} s u m\left(|\mathbf{W}|^{2}\right)+I m^{2}\left(\mathbf{W}^{*} \mathbf{T}_{\mathbf{q}_{00}}\right) \\
& +\left.\sum_{k=1}^{K}\left|\mathbf{W}^{*} \mathbf{T}_{\left.\mathbf{q}_{k 0}\right|^{2}}+\sum_{n=1}^{N} \sum_{k=-K^{-}}^{K}\right| \mathbf{W}^{* \mathbf{T}} \mathbf{q}_{\mathbf{k n}}\right|^{2}
\end{aligned}
$$

With this change, $\phi(s)$ simply becomes

$$
\phi(s)=\frac{1}{2} \sigma_{0}^{2} s^{2}-A_{00} s-\ln (s)
$$

Solving for the positive root of the quadratic $\phi^{\prime}(s)=\sigma_{0}^{2} s-A_{00}-s^{-1}=0$ then yields the starting value

$$
s_{0}=\frac{1}{2 \sigma_{0}^{2}}\left[A_{00}+\left(A_{00}^{2}+4 \sigma_{0}^{2}\right)^{\frac{1}{2}}\right]
$$

Newton's method can then applied until the change in $s_{0}$ becomes negligible ${ }^{55}$.

Recalling equation 5.2.5 and setting $s=s_{0}+j y$ the integral to be evaluated is [59]

$$
P_{b}=\int_{0}^{\infty} G\left(s_{0}+j y\right) d y
$$

where

$$
G(s)=\frac{1}{\pi} \operatorname{Re}\left(s^{-1} g(s)\right)=\frac{1}{\pi} \operatorname{Re}\left(s^{-1} g_{\eta}(s) g_{\xi}(s) e^{-A_{[x \mid s}}\right)
$$

The trapezoidal rule is used to approximate the integral as

$$
P_{b}=\Delta y\left[\frac{1}{2} G\left(s_{0}\right)+\sum_{u=1}^{U} G\left(s_{0}+j u \Delta y\right)\right]
$$

35 This should typically require only a few $(<5)$ iterations. 
where $U$ is large enough such that $G\left(s_{0}+j u y\right)$ is negligible for $u>U$. One can start with a step size [59] of $\Delta y=\left[\phi^{\prime \prime}\left(s_{0}\right)\right]^{-\frac{1}{2}}$ and recompute equation 5.23 until desired accuracy is obtained.

By generating a large number of independent channel sets, statistically significant outage and average error rates can thus be obtained. The generation of the (e.g. 10000) independent sets of $h_{n i}(t) \quad i=1, \ldots M ; \quad n=0, \ldots . N$ will be done according to the 9-tap model discussed in Chapter 3 and also by the cluster model [60] alluded to in Chapter 2 and which is deemed more representative of typical indoor channels. For each set of channel realizations $h_{n i}(t), i=1, \ldots M ; \quad n=0, \ldots N$, the procedure to find the uncoded $P_{b}$ thus involves the following steps:

1. Find all the $q_{k n} k=-K, \ldots K ; n=0, \ldots N$ (eq. 3.19) according to equations 3.14 and 3.15 .

2. Find the interference-plus-noise covariance matrix $C$ (eq. 3.34) from equations 3.23 and 3.30 .

3. Find the tap weight vector $\mathbf{W}_{\text {opt }}=\mathbf{C}^{-1} \mathbf{q}_{00}$ (eq. 3.36) and also all the $\mathbf{W}^{*} \mathbf{T}_{\mathbf{q} \mathbf{n}} \quad k=-K, \ldots K, n=0, \ldots N$.

4. Compute the saddlepoint search starting point from equations 5.18 and 5.20. Then, find the saddlepoint from equations 5.14 through 5.17 .

5. Use the trapezoidal rule to find $P_{b}$ as per equation 5.23.

Note that all the preceding expressions hold for QPSK while for BPSK, all $B_{k n}$ terms are set to zero, i.e. there is no cross-rail interference.

\subsubsection{Coded BER}

Recall that the bit error rate upper bound for soft-decision Viterbi decoding of a rate $1 / n$ convolutional code can be expressed as $P_{b}=\sum_{d=d_{\text {free }}}^{\infty} \beta_{d} P_{2}(d)$ where $\beta_{d}$ is obtained from the first derivative of the code's transfer function and $P_{2}(d)$ is the pairwise error probability for sequences that differ in $d$ bits. In terms of the notation of equation 5.2 , 
the pairwise error probability can thus be expressed as ${ }^{56}$

$$
P_{2}(d)=\operatorname{Pr}\left(y=\sum_{i=0}^{d-1} \operatorname{Re}\left(z_{i}\right) \leq 0 \mid a_{00}=a_{10}=\ldots=a_{(d-1) 0}=1\right)
$$

for the BPSK case or as

$$
P_{2}(d)= \begin{cases}\operatorname{Pr}\left(\sum_{i=0}^{\frac{d}{2}-1}\left(\operatorname{Re}\left(z_{i}\right)+\operatorname{Im}\left(z_{i}\right) \leq 0 \mid s_{i 0}=1+j\right)\right) & \text { d even } \\ \operatorname{Pr}\left(\sum_{i=0}^{\frac{(d-1)}{2}-1}\left(\operatorname{Re}\left(z_{i}\right)+\operatorname{Im}\left(z_{i}\right)+\operatorname{Re}\left(\left.z_{\left.\frac{(d-1)}{2}\right) \leq 0 \mid}\right|_{a_{\frac{(d-1)}{2} 0}=1} ^{s_{i 0}=1+j}\right)\right)\right. & d \text { odd }\end{cases}
$$

for the QPSK case. The steps required to evaluate the BPSK/QPSK pairwise and thus bit error probabilities will now be described for both the ideally-interleaved and non-interleaved cases.

\subsubsection{Ideal interleaving (random errors)}

Assuming that successive symbols fade independently which is equivalent to interleaving of infinite depth (i.e. random errors), the successive bits $z_{i}$ appearing at the Viterbi decoder input have all been subjected to independent fading. In such a case and assuming symmetry between the two rails, one may consider

$$
\begin{aligned}
& y= \sum_{i=0}^{d-1} \operatorname{Re}\left(z_{i}\right) \\
&=\sum_{i=0}^{d-1}\left\{a_{00} A_{00 i}+\sum_{k=1}^{K} a_{k 0} A_{k n i}-\sum_{k=0}^{K} b_{k 0} B_{k: n i}\right. \\
&\left.\quad+\sum_{n=1}^{N} \sum_{k=-K}^{K}\left[a_{k n} A_{k n i}-b_{k n} B_{k n i}\right]+\operatorname{Re}\left(\mathbf{W}_{i}^{*} \mathrm{~T} \mathbf{V}\right)\right\}
\end{aligned}
$$

as the decision variate for both BPSK and QPSK ${ }^{57}$. As in the uncoded case, the only distinction is that all $B_{k n}$ terms are equal to zero for the BPSK case.

\footnotetext{
56 Recall from Chapter 3 (eq. 3.57) that one typically assumes the all-zero path to be correct. The case of opposite polarity is used here. The distinction is however irrelevant.

57 It is assumed here that successive bits are interleaved at the encoder output and de-interleaved at the input to the (Viterbi) decoder. Hence, even for QPSK, successive bits undergo independent fading.
} 
The determination of the (e.g. 10000) individual $P_{b}$ 's can be done similarly to the uncoded case. However, instead of generating one set of $h_{m}(t) i=1, \ldots M: n=$ $0, \ldots N$, one generates $\left(d_{\text {free }}+D\right)$ such sets where $D$ is such that $P_{2}\left(d_{\text {free }}+D\right) \ll$ $P_{2}\left(d_{\text {free }}\right)$, i.e. $P_{b}=\sum_{d=d_{\text {free }}}^{d_{\text {free }}+D} \beta_{d} P_{2}(d)$. Each pairwise error probability is then evaluated using essentially the same technique as in the uncoded case with the moment-generating function of $y=\sum_{i=0}^{d-1} \operatorname{Re}\left(z_{i}\right)$ equal to

$$
g(s)=g_{\eta}(s) g_{\xi}(s) \prod_{i=0}^{d-1} \exp \left(-A_{00 i} s\right)
$$

where 58

$$
g_{\xi}(s)=\prod_{i=0}^{d-1} \exp \left(\frac{N_{0} s^{2}}{4 R_{c}} \operatorname{sum}\left(\left|\mathbf{W}_{\mathrm{i}}\right|^{2}\right)\right)=\exp \left(\frac{N_{0} s^{2}}{4 R_{c}} \sum_{i=0}^{d-1} \operatorname{sim}\left(\left|\mathrm{W}_{\mathrm{i}}\right|^{2}\right)\right)
$$

and

$$
g_{\eta}(s)=\prod_{i=0}^{d-1} g_{\eta i}(s)
$$

in which each of the $d$ interference mgf's are obtained from equation 5.8. The saddlepoint is determined via the function

$$
\begin{aligned}
& \phi(s)=\sum_{i=0}^{d-1}\left\{\sum_{k=1}^{K} \ln \left[\cosh \left(A_{k 0 i} s\right)\right]+\sum_{k=0}^{K} \ln \left[\cosh \left(B_{k 0,} s\right)\right]\right. \\
& +\sum_{n=1}^{N} \sum_{k=-K^{-}}^{K}\left(\ln \left[\cosh \left(A_{k \cdot n z} s\right)\right]+\ln \left[\cosh \left(B_{k m, s} \cdot\right]\right)\right. \\
& \left.+\frac{N_{0}}{4} s^{2} \operatorname{sum}\left(\left|\mathbf{W}_{\mathrm{i}}\right|^{2}\right)-A_{00 ;} s\right\}-\ln (. s)
\end{aligned}
$$

and its first two derivatives

$$
\begin{aligned}
\phi^{\prime}(s) & =\sum_{i=0}^{d-1}\left\{\sum_{k=1}^{K} A_{k 0_{i}} \tanh \left(A_{k 0 i} s\right)+\sum_{k=0}^{K} B_{k \cdot()_{i}} \tanh \left(B_{k()_{2} s} s\right)\right. \\
& +\sum_{n=1}^{N} \sum_{k=-K}^{K}\left(A_{k n i} \tanh \left(A_{k n i} s\right)+B_{k n i} \tanh \left(B_{k n i} s\right)\right) \\
& \left.+\frac{N_{0}}{2} s \operatorname{sum}\left(\left|W_{i}\right|^{2}\right)-A_{0 n_{h}}\right\}-s^{-1}
\end{aligned}
$$

58 In the coded case, the noise variance per transmitted (coded) bit is now $\frac{N .1}{R_{c}}$. 
and

$$
\begin{aligned}
\phi^{\prime \prime}(s) & =-\sum_{i=0}^{d-1}\left\{\sum_{k=1}^{h} A_{k 0 i}^{2} \operatorname{sech}^{2}\left(A_{k n i} s\right)+\sum_{k=0}^{K} B_{k n_{i}}^{2} \operatorname{sech}^{2}\left(B_{k n i} s\right)\right. \\
& +\sum_{n=1}^{N} \sum_{k=-K^{*}}^{K}\left(A_{k n i}^{2} \operatorname{sech}^{2}\left(A_{k n i} s\right)+B_{k n i}^{2} \operatorname{sech}^{2}\left(B_{k n i} s\right)\right) \\
& \left.+\frac{N_{0}}{2} \operatorname{sum}\left(\left|\mathbf{W}_{i}\right|^{2}\right)\right\}+s^{-2}
\end{aligned}
$$

Replacing, as in the uncoded case, the interference terms in eq. 5.26 by gaussian noise yields a total variance of

$$
\begin{aligned}
\sigma_{0}^{2}=\sum_{i=0}^{d-1}\{ & \frac{N_{0}}{2} \operatorname{sum}\left(\left|\mathbf{W}_{\mathrm{i}}\right|^{2}\right)+B_{00 i}^{2} \\
& \left.+\sum_{k=1}^{K}\left(A_{k 0 i}^{2}+B_{k 0 i}^{2}\right)+\sum_{n=1}^{N} \sum_{k=-K}^{K}\left(A_{k n i}^{2}+B_{k n i}^{2}\right)\right\}
\end{aligned}
$$

from which the saddlepoint search starting pcint is found to be

$$
s_{0}=\frac{1}{2 \sigma_{0}^{2}}\left[\sum_{i=0}^{d-1} A_{00 i}+\left(\left(\sum_{i=0}^{d-1} A_{00_{i}}\right)^{2}+4 \sigma_{0}^{2}\right)^{\frac{1}{2}}\right]
$$

\subsubsection{No interleaving (burst-error case)}

On the other hand, if one assumes that there is no interleaving (burst-error case) as is considered for this slow-fading application, only one set of $h_{n i}(t), i=1, \ldots M ; n=$ $0, \ldots N$ need be generated to compute each of the (10000) $P_{b}$ 's. However, the determination of the $\mathrm{mgf}$ required for each $P_{2}(d)$ is now far more complicated as the various symbols do not fade independently and interfere with each other because of symbol dispersion. The analysis can be made tractable by assuming that the $d$ bits that differ between the two paths under consideration for a given $P_{2}(d)$ are successive bits. Since $P_{2}(d)$ is highest for small $d$ where the length (in terms of coded bits) of a path merging with the all-zero path is generally only slightly larger than $d$, the resulting performance estimate should not be affected greatly by this approximation of successive erroneous bits. As an example, the rate $(1 / n)=1 / 2$, 
$d_{\text {free }}=5$, constraint length 3 code used in Chapters 3 and 4 has transfer function $T(J, N, D)=J^{3} N D^{5}+2 J^{4} N^{2} D^{6}+4 J^{5} N^{3} D^{7}+\ldots$ where the exponent of $J$ denotes the number of trellis branches, that of $N$ the number of information bits equal to 1 . and that of $D$ the number of coded bits equal to 1 on the path merging with the allzero path. It is seen that for $d=d_{\text {free }}=5$ where $P_{2}(d)$ is highest, the path has $3 \times n=3 \times 2=6$ coded bits of which 5 are in error if an incorrect choice between the two paths is made. As $d$ increases, the successive bit approximation degrades somewhat but simultaneously, $P_{2}(d)$ becomes smaller and eventually negligible with respect to $P_{2}\left(d_{\text {free }}\right)$. In addition, this successive erroneous bit approximation is all the more realistic given the quasi-static nature of the channel. The BPSK and QPSK cases will now be discussed separately as per equations 5.24 and 5.25 .

\section{BPSK}

To determine the pairwise error probabilities $P_{2}(d), d=d_{\text {free }}, \ldots, d_{\text {free }}+D$ for BPSK, one must thus determine the mgf of the following variate

$$
\begin{aligned}
y & =\sum_{i=0}^{d-1} \operatorname{Re}\left(z_{i}\right) \\
& =\sum_{i=0}^{d-1} \sum_{k=0}^{K} A_{k 0} a_{(k+i) 0}+\sum_{n=1}^{N} \sum_{i=0}^{d-1} \sum_{k=-K}^{K} A_{k n} a_{(k+i) n}+\sum_{i=0}^{d-1} \operatorname{Rc}\left(\mathbf{W}^{*} \mathbf{T} \mathbf{V}_{\mathbf{i}}\right)
\end{aligned}
$$

where, as before, $A_{k n}=\sqrt{\frac{I_{n}}{S}} \operatorname{Re}\left(\mathbf{W}^{* \mathbf{T}} \mathbf{q}_{\mathbf{k n}}\right)$. The mgf of $y$ consists of the AWGN mgf $g_{\xi}(s)$, the signal $(n=0) \mathrm{mgf}^{59} g_{\lambda}(s)$, and the cochannel interference $(n=1, \ldots, N)$ mgf $g_{\eta}(s)$ i.e.

$$
g(s)=g_{\lambda}(s) g_{\eta}(s) g_{\xi}(s)
$$

The gaussian noise is uncorrelated from one symbol interval to the next yielding

$$
g_{\xi}(s)=\prod_{i=0}^{d-1} \exp \left(\frac{N_{0} s^{2}}{4 R_{c}} \operatorname{sum}\left(|\mathbf{W}|^{2}\right)\right)=\exp \left(\frac{d N_{0} s^{2}}{4 R_{c}} \operatorname{sum}\left(|\mathbf{W}|^{2}\right)\right)
$$

This includes the effect of intersymbol interference. 
As for $g_{\lambda}(s)$, it should be noted that the $d$ bits equal to 1 , i.e. $a_{0 B}, \ldots, a_{(d-1) 0}=1$, will generate exponential instead of cosh terms in the signal mgf which is therefore ${ }^{60}$

$$
g_{\lambda}(s)= \begin{cases}\prod_{k=0}^{K} \exp \left(-\sum_{l=0}^{k} A_{l 0} s\right) \prod_{k=K^{\prime}+1}^{d-1} \exp \left(-\sum_{l=0}^{K} A_{l 0} s\right) & \\ \prod_{k=d}^{(d+K-1)} \cosh \left(\sum_{l=k-(d-1)}^{K} A_{l 0} s\right) & \text { for } d>K \\ \prod_{k=0}^{d-1} \exp \left(-\sum_{l=0}^{k} A_{l 0} s\right) \prod_{k=d}^{K} \cosh \left(\sum_{l=k-(d-1)}^{k} A_{l n} s\right) & \\ \prod_{k=K^{k}+1}^{(d+K-1)} \cosh \left(\sum_{l=k-(d-1)}^{K} A_{l n} s\right) & \text { for } d \leq K\end{cases}
$$

Similarly, the CCl mgf is found to be

$$
\begin{aligned}
g_{\eta}(s)= & \prod_{n=1}^{N} \prod_{k=-K}^{(d-K-1)} \cosh \left(\sum_{l=-K}^{k} A_{l n} s\right) \\
& \prod_{k=d-K}^{K} \cosh \left(\sum_{l=k-(d-1)}^{k} A_{l n} s\right) \prod_{k=K^{\prime}+1}^{d+K-1} \cosh \left(\sum_{l=k-(d-1)}^{K} A_{l n} s\right)
\end{aligned}
$$

The function $\phi(s)=\ln \left(s^{-1} g(s)\right)$ is now found to be

$$
\phi(s)= \begin{cases}\sum_{k=0}^{K}\left(-\sum_{l=0}^{k} A_{l 0} s\right)+\sum_{k=K+1}^{d-1}\left(-\sum_{l=0}^{K} A_{l 0} s\right) & \text { for } d>K \\ +\sum_{k=d}^{(d+K-1)} \ln \left(\cosh \left(\sum_{l=k-(d-1)}^{K} A_{l 0} s\right)\right)+\phi_{1}(s) & \\ \sum_{k=0}^{(d-1)}\left(-\sum_{l=0}^{k} A_{l 0} s\right)+\sum_{k=d}^{K} \ln \left(\cosh \left(\sum_{l=k-(d-1)}^{k} A_{l 0} s\right)\right) & \\ +\sum_{k=K^{K}+1}^{(d+K-1)} \ln \left(\cosh \left(\sum_{l=k-(d-1)}^{K} A_{l 0} s\right)\right)+\phi_{1}(s) & \text { for } d \leq K\end{cases}
$$

The expressions for the signal and CCI mgf's may not be obvious to the reader. One may be convinced of these results by taking a few examples, collecting terms for each bit and determining the required means. 
where

$$
\begin{aligned}
& \phi_{1}(s)=\sum_{n=1}^{N}\left\{\sum_{k=-K^{\prime}}^{(d-K-1)} \ln \left(\cosh \left(\sum_{l=-K^{*}}^{k} A_{l n} s\right)\right)\right. \\
& +\sum_{k=d-K}^{K} \ln \left(\cosh \left(\sum_{l=k-(d-1)}^{k} A_{l n} s\right)\right) \\
& \left.+\sum_{k=K^{\prime}+1}^{(d+K-1)} \ln \left(\cosh \left(\sum_{l=k-(d-1)}^{K} A_{l n} s\right)\right)\right\}+\frac{d N_{0} s^{2}}{4} \cdot s \ln \left(|\mathrm{W}|^{2}\right)-\ln (s)
\end{aligned}
$$

Its first derivative is then

$$
\phi^{\prime}(s)=\left\{\begin{array}{l}
\phi_{1}^{\prime}(s)-\sum_{k=0}^{K} \sum_{l=0}^{k} A_{l 0}-\sum_{k=K+1}^{d-1} \sum_{l=0}^{K} A_{l 0} \\
+\sum_{k=d}^{(d+K-1)}\left[\left(\sum_{l=k-(d-1)}^{K} A_{l 0}\right) \tanh \left(\sum_{l=k-(d-1)}^{K} A_{l 0} \cdot s\right)\right] \quad ; \text { for } d>K \\
\phi_{1}^{\prime}(s)-\sum_{k=0}^{(d-1)} \sum_{l=0}^{k} A_{l 0} s+\sum_{k=d}^{K}\left[\left(\sum_{l=k-(d-1)}^{k} A_{l 0}\right) \tanh \left(\sum_{l=k-(d-1)}^{k} A_{l 0^{s}}\right)\right] \\
+\sum_{k=K+1}^{(d+K-1)}\left[\left(\sum_{l=k-(d-1)}^{K} A_{l 0}^{K}\right) \tanh \left(\sum_{l=k-(d-1)}^{K} A_{l 0} s\right)\right]
\end{array}\right.
$$

where

$$
\begin{aligned}
& \phi_{1}^{\prime}(s)=\sum_{n=1}^{N}\left\{\sum_{k=-K^{K}}^{(d-K-1)}\left[\left(\sum_{l=-K^{\prime}}^{k} A_{l n}\right) \tanh \left(\sum_{l=-K^{\prime}}^{k} A_{l n} s\right)\right]\right. \\
& +\sum_{k=d-K}^{K}\left[\left(\sum_{l=k-(d-1)}^{k} A_{l n}\right) \tanh \left(\sum_{l=k-(d-1)}^{k} A_{l n} s\right)\right] \\
& \left.+\sum_{k=K+1}^{(d+K-1)}\left[\left(\sum_{l=k-(d-1)}^{K} A_{l n}\right) \tanh \left(\sum_{l=k-(d-1)}^{K} A_{l n} s\right)\right]\right\}+\frac{d N_{(j) s}}{2} \operatorname{surn}\left(|W|^{2}\right)-s^{-1}
\end{aligned}
$$


and its second derivative is finally

$$
\begin{aligned}
& \phi^{\prime \prime}(s)= \\
& \left\{\begin{array}{l}
\sum_{k=d}^{(d+k-1)}\left[\left(\sum_{l=k-(d-1)}^{K} A_{l 0}\right)^{2} \operatorname{sech}^{2}\left(\sum_{l=k-(d-1)}^{K} A_{l n} s\right)\right]+\phi_{1}^{\prime \prime}(s) \quad \text { for } d>K \\
\sum_{k=d}^{K}\left[\left(\sum_{l=k-(d-1)}^{k} A_{l 0}\right)^{2} \operatorname{sech}^{2}\left(\sum_{l=k-(d-1)}^{k} A_{l 0} s\right)\right] \\
+\sum_{k=K^{\prime \prime}+1}^{(d+K-1)}\left[\left(\sum_{l=k-(d-1)}^{K} A_{l 0}\right)^{2} \operatorname{sech}^{2}\left(\sum_{l=k-(d-1)}^{K} A_{l 0} s\right)\right]+\phi_{1}^{\prime \prime}(s) \text { for } d \leq K^{-}
\end{array}\right.
\end{aligned}
$$

where

$$
\begin{aligned}
& \phi_{1}^{\prime \prime}(s)=\sum_{n=1}^{N}\left(\sum_{k=-K^{\prime}}^{(d-K-1)}\left[\left(\sum_{l=-K^{\prime}}^{k} A_{l n}\right)^{2} \operatorname{sech}^{2}\left(\sum_{l=-K}^{k} A_{l n} s\right)\right]\right. \\
& +\sum_{k=d-K}^{K}\left[\left(\sum_{l=k-(d-1)}^{k} A_{l n}\right)^{2} \operatorname{sech}^{2}\left(\sum_{l=k-(d-1)}^{k} A_{l n} s\right)\right] \\
& \left.+\sum_{k=K^{\prime}+1}^{(d+K-1)}\left[\left(\sum_{l=k-(d-1)}^{K} A_{l n}\right)^{2} \operatorname{sech}^{2}\left(\sum_{l=k-(d-1)}^{K} A_{l n} s\right)\right]\right\}+\frac{d N_{0}}{2} \operatorname{sum}\left(|W|^{2}\right)+s^{-2}
\end{aligned}
$$

Replacing the interference terms ${ }^{61}$ in equation 5.35 by gaussian noise of equal variance yields a total variance of

$$
\sigma_{0}^{2}=d \frac{N_{0}}{2} s u m\left(|\mathbf{W}|^{2}\right)+\sum_{i=0}^{d-1} \sum_{k=K-i}^{K} A_{k 0}^{2}+\sum_{n=1}^{N} d \sum_{k=-K}^{K} A_{k n}^{2}
$$

62 Notice that the first term in equation 5.35 consists of a desired signal term $\sum_{i=0}^{d-1} \sum_{k=0}^{K-(i+1)} A_{k 0} a_{(k+i) 0}$ and an intersymbol interference term $\sum_{i=1}^{d-1} \sum_{k=K-i}^{K} A_{k 0} a_{(k+i) n}$. 
The saddlepoint search starting point $s_{0}$ is computed similarly to the uncoded case (eq. 5.20) except that $A_{00}$ is replaced by $\sum_{i=0}^{d-1} \sum_{k=0}^{k-(i+1)} A_{k-0}$, i.e.

$$
s_{0}=\frac{1}{2 \sigma_{0}^{2}}\left[\sum_{i=0}^{d-1} \sum_{k=0}^{k-(i+1)} A_{k 0}+\left(\left(\sum_{i=0}^{d-1} \sum_{k=0}^{K-(i+1)} A_{k 0}\right)^{2}+4 \sigma_{0}^{2}\right)^{\frac{1}{2}}\right]
$$

\section{QPSK}

For QPSK, evaluating $P_{2}(d)$ (equation 5.25) requires the $\mathrm{mgf}$ of the following variate

$$
y= \begin{cases}\sum_{i=0}^{d_{e}-1}\left(\operatorname{Re}\left(z_{i}\right)+\operatorname{Im}\left(z_{i}\right)\right) & d \text { cren } \\ \sum_{i=0}^{d_{0}-1}\left(\operatorname{Re}\left(z_{i}\right)+\operatorname{Im}\left(z_{i}\right)\right)+\operatorname{Re}\left(z_{d_{0}}\right) & d \text { odd }\end{cases}
$$

where $d_{e}=\frac{d}{2}$ and $d_{o}=\frac{d-1}{2}$. Initially, the $X_{k n}$ and $Y_{k n}$ factors are defined as

$$
\begin{aligned}
& \boldsymbol{X}_{k n} \triangleq A_{k n}+B_{k n}=\sqrt{\frac{I_{n}}{S}}\left(\operatorname{Re}\left(\mathbf{W}^{* \mathbf{T}_{\mathbf{q} \mathbf{k n}}}\right)+\operatorname{Im}\left(\mathbf{W}^{* \mathbf{T}_{\mathbf{q} \mathbf{}}}\right)\right) \\
& Y_{k n} \triangleq A_{k n}-B_{k n}=\sqrt{\frac{I_{n}}{S}}\left(\operatorname{Re}\left(\mathbf{W}^{* \mathbf{T}_{\mathbf{q} \mathbf{k}}}\right)-\operatorname{Im}\left(\mathbf{W}^{* \mathbf{T}_{\mathbf{q} \mathbf{n}}}\right)\right)
\end{aligned}
$$

Expanding the various $z_{i}$ terms in equation 5.48 then yields for $d$ even

$$
\begin{aligned}
y= & \sum_{i=0}^{d_{e}-1} \sum_{k=0}^{K}\left[\mathbf{X}_{k 0} a_{(k+i) 0}+Y_{k o} b_{(k+i) 0}\right]+\sum_{n=1}^{N} \sum_{i=0}^{d_{e}-1} \sum_{k=-K^{K}}^{K}\left[X_{k n}(i k+i) n+Y_{k n} b_{(k+1) n}\right] \\
& +\sum_{i=0}^{d_{e}-1}\left[\operatorname{Re}\left(\mathbf{W}^{*} \mathbf{T}_{\mathbf{i}}\right)+\operatorname{Im}\left(\mathbf{W}^{*} \mathbf{T} \mathbf{V}_{\mathbf{i}}\right)\right]
\end{aligned}
$$

and for $d$ odd,

$$
\begin{aligned}
y= & \sum_{i=0}^{d_{o}} \sum_{k=0}^{K} X_{k 0} a_{(k+i) 0}+\sum_{i=0}^{d_{o}-1} \sum_{k=0}^{K} Y_{k 0} b_{(k+i) 0} \\
& +\sum_{n=1}^{N}\left[\sum_{i=0}^{d_{o}} \sum_{k=-K}^{K} X_{k n} a_{(k+i) n}+\sum_{i=0}^{d_{o}-1} \sum_{k=-K^{K}}^{K} Y_{k n} b_{(k+i) n}\right] \\
& +\sum_{i=0}^{d_{o}} R_{\epsilon}\left(\mathbf{W}^{*} \mathbf{T} \mathbf{V}_{\mathbf{i}}\right)+\sum_{i=0}^{d_{o}-1} I m\left(\mathbf{W}^{*} \mathbf{T} \mathbf{V}_{\mathbf{i}}\right)
\end{aligned}
$$


For $d$ even, the mgf $g(s)=g_{\lambda}(s) g_{\xi}(s)$ is now subjected to the condition $a_{00}, b_{00}, \ldots, a_{\left(d_{e}-1\right) 0}, b_{\left(d_{t}-1\right) 0}=1$ while for $d$ odd, the condition is $a_{00}, b_{n 0}, \ldots, a_{\left(d_{o}-1\right) a}, b_{\left(d_{0}-1\right) 0}, a_{d_{o} 0}=1$. The AWGN mgf remains equal to

$$
g_{\xi}(s)=\exp \left(\frac{d N_{0} s^{2}}{4 R_{c}} \operatorname{sum}\left(|\mathbf{W}|^{2}\right)\right)
$$

while the CCI $\mathrm{mgf}$ is for $d$ even

$$
\begin{aligned}
g_{\eta}(s)=\prod_{n=1}^{N}\{ & \prod_{k=-K^{k}}^{\left(d_{e}-K^{K}-1\right)}\left[\cosh \left(\sum_{l=-K^{\prime}}^{k} X_{l n} s\right) \cosh \left(\sum_{l=-K^{K}}^{k} Y_{l n} s\right)\right] \\
& \cdot \prod_{k=d_{e}-K}^{K}\left[\cosh \left(\sum_{l=k-\left(d_{e}-1\right)}^{k} X_{l n} s\right) \cosh \left(\sum_{l=k-\left(d_{e}-1\right)}^{k} Y_{l n} s\right)\right] \\
& \left.\cdot \prod_{k=K^{+}+1}^{\left(d_{e}+K^{\prime}-1\right)}\left[\cosh \left(\sum_{l=k-\left(d_{e}-1\right)}^{K} X_{l n} s\right) \cosh \left(\sum_{l=k-\left(d_{e}-1\right)}^{K} Y_{l n} s\right)\right]\right\}
\end{aligned}
$$

and for $d$ odd,

$$
\begin{aligned}
g_{\eta}(s)=\prod_{n=1}^{N}\left\{\prod_{k=-K}^{\left.i d_{o}-K\right)}\left[\cosh \left(\sum_{l=-K}^{k} X_{l n} s\right) \prod_{k=-K}^{\left(d_{o}-K-1\right)} \cosh \left(\sum_{l=-K}^{k} Y_{l n} s\right)\right]\right. \\
\prod_{k=d_{o}-K^{\prime}+1}^{K}\left[\cosh \left(\sum_{l=k-d_{o}}^{k} X_{l n} s\right) \prod_{k=d_{o}-K}^{K} \cosh \left(\sum_{l=k-\left(d_{o}-1\right)}^{k} Y_{l n} s\right)\right] \\
\left.\prod_{k=K^{\prime}+1}^{\left(d_{o}+K^{\prime}\right)}\left[\cosh \left(\sum_{l=k-d_{o}}^{K} X_{l n} s\right) \prod_{k=K^{\prime}+1}^{\left(d_{o}+K-1\right)} \cosh \left(\sum_{l=k-\left(d_{o}-1\right)}^{K} Y_{l n} s\right)\right]\right\}
\end{aligned}
$$

The two preceding equations, representing the moment-generating function of the $n=1, \ldots . N$ terms in equations 5.49 and 5.50 , were obtained by carefully collecting terms of the form $\varepsilon[\exp (-s(\cdots))]$ for each of the $a_{k n}$ and $b_{k n}$ bits. Applying the same procedure to the $n=0$ terms and incorporating the condition of the first $d$ bits set to 1 , 
the signal mgf $g_{\lambda}(s)$ is found to be, for the case of $d$ odd

$$
\begin{aligned}
& \left(\prod_{k=0}^{h} \exp \left(-\sum_{l=0}^{k} 2 A_{l 0} s\right) \prod_{k=h^{k}+1}^{\left(d_{0}-1\right)} \exp \left(-\sum_{l=0}^{h} 2 A_{(0)} s-\sum_{l=0}^{d_{l}} \mathrm{r}_{l 0} \cdot s\right)\right.
\end{aligned}
$$

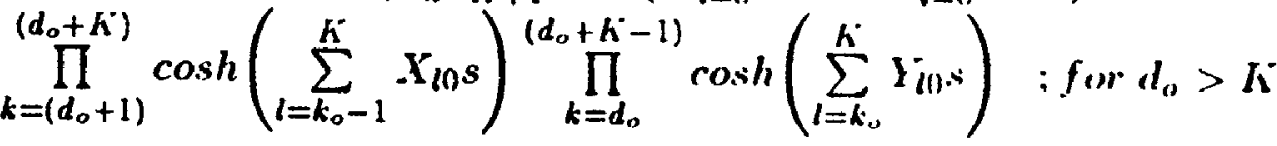

$$
\begin{aligned}
& \prod_{k=0}^{\left(d_{0}-1\right)}\left[\exp \left(-\sum_{l=0}^{k} 2 A_{l n} s-\sum_{l=0}^{d_{o}} X_{l n} s\right) \cosh \left(\sum_{l=1}^{d_{0}} Y_{i n s} s\right)\right] \\
& g_{\lambda}(s)=\left\{\prod_{k=d_{o}+1}^{2 d_{0}} \cosh \left(\sum_{l=k_{0}-1}^{d_{0}} X_{l 0} s\right)_{k=d_{o}+1}^{\left(2 d_{o}-1\right)} \cosh \left(\sum_{l=k_{o}}^{d_{0}} Y_{l 0} \cdot s\right): \text { for } d_{o}=K\right. \\
& \prod_{k=d_{0}+1}^{K} \cosh \left(\sum_{l=k_{0}-1}^{k} X_{l n s} \prod_{k=k^{\prime}+1}^{\left(d_{o}+k^{\prime}\right)} \cosh \left(\sum_{l=k_{b-1}-1}^{K} I_{l 0^{*}}\right)\right. \\
& \prod_{k=d_{o}}^{K} \cosh \left(\sum_{l=k_{0}}^{k} Y_{l 0} s\right)_{k=K^{\prime}+1}^{\left(d_{0}+K^{\prime}-1\right)} \cosh \left(\sum_{i=k_{0}}^{K} Y_{()^{i}}\right) \\
& \prod_{k=0}^{\left(d_{o}-1\right)} \exp \left(-\sum_{l=0}^{k} 2 A_{l 0} s-\sum_{l=0}^{d_{0}} X_{l 0} s\right) \quad ; \quad \text { for } d_{o}<K^{\circ} \\
& \text { where } k_{o}=k-\left(d_{o}-1\right), \quad d_{o}=\frac{(d-1)}{2} \text { and } d \text { is odd. }
\end{aligned}
$$

or for $d$ even,

$$
\begin{aligned}
& \prod_{k=d_{e}}^{\left(d_{e}+K^{-1)}\right.}\left[\cosh \left(\sum_{l=k_{e}}^{K} X_{l 0} s\right) \cosh \left(\sum_{l=k_{e}}^{K} Y_{l 0} s\right)\right] \\
& \prod_{k=0}^{h} \exp \left(-\sum_{l=0}^{k} 2 A_{10} s\right) \prod_{k=K+1}^{\left(d_{e}-1\right)} \exp \left(-\sum_{l=0}^{K} 2 A_{l 0} s\right) \quad: \text { for } d_{k}>K \\
& g_{\lambda}(s)=\{ \\
& \prod_{k=d_{e}}^{k}\left[\cosh \left(\sum_{l=k_{e}}^{k} X_{l 0} s\right) \cosh \left(\sum_{l=k_{e}}^{k} Y_{l 0} s\right)\right] \prod_{k=0}^{\left(d_{i}-1\right)} \exp \left(-\sum_{l=0}^{k} 2 A_{l 0} s\right) \\
& \prod_{k=K^{\prime}+1}^{\left(d_{e}+K^{\prime}-1\right)}\left[\cosh \left(\sum_{l=k_{e}}^{K} X_{l 0} s\right) \cosh \left(\sum_{l=k_{e}}^{K} Y_{l 0^{s}}\right)\right] \quad ; \text { for } d_{e} \leq K \\
& \text { where } k_{e}=k-\left(d_{e}-1\right), \quad d_{e}=\frac{d}{2} \quad \text { and } \quad d \text { is } \text { m. }
\end{aligned}
$$


As $g_{\lambda}(s)$ is represented by five different expressions, so is the function 0.$)=$ $\ln \left(s^{-1} g(s)\right)$ and its first two derivatives. For $d$ even and with the function $\phi_{l e}(s)$ defined as

$$
\begin{aligned}
& \phi_{1 e}(s)=\frac{d N_{0} s^{2}}{4} s u m\left(|W|^{2}\right)-\ln (s) \\
& \sum_{n=1}^{N}\left(\sum_{k=-K^{-}}^{\left(d_{e}-K^{-}-1\right)} \ln \left(\cosh \left(\sum_{l=-K^{-}}^{k} X_{l n} s\right)\right)+\sum_{k=-K^{-}}^{\left(d_{e}-K^{k}-1\right)} \ln \left(\cosh \left(\sum_{l=-K^{-}}^{l} Y_{l n} s\right)\right)\right. \\
& +\sum_{k=d_{e}-K^{\prime}}^{K} \ln \left(\cosh \left(\sum_{i=k-\left(d_{e}-1\right)}^{k} X_{l n} s\right)\right)+\sum_{k=d_{e}-K^{\prime}}^{K} \ln \left(\cosh \left(\sum_{l=k-\left(d_{e}-1\right)}^{k} Y_{l n} s\right)\right) \\
& +\sum_{k=K^{\prime}+1}^{\left(d_{e}+K^{\prime}-1\right)} \ln \left(\cosh \left(\sum_{l=k-\left(d_{e}-1\right)}^{K} X_{l n} s\right)\right) \\
& \left.+\sum_{k=k+1}^{\left(d_{e}+K-1\right)} \ln \left(\cosh \left(\sum_{l=k-\left(d_{e}-1\right)}^{K} Y_{l n} s\right)\right)\right\}
\end{aligned}
$$

we obtain

$$
\begin{aligned}
& \phi(s)=\left\{\begin{array}{l}
-\sum_{k=0}^{K} \sum_{l=0}^{k} 2 A_{l 0} s-\sum_{k=K^{+}+1}^{\left(d_{e}-1\right)} \sum_{l=0}^{K} 2 A_{l 0} s+\sum_{k=d_{e}}^{\left(d_{e}+K-1\right)} \ln \left(\cosh \left(\sum_{l=k_{e}}^{K} X_{l 0} s\right)\right) \\
+\sum_{k=d_{e}}^{\left(d_{e}+K^{-}-1\right)} \ln \left(\cosh \left(\sum_{l=k_{e}}^{K} Y_{l 0} s\right)\right)+\phi_{1 e}(s) \quad \text { for } d_{e}>K \\
-\sum_{k=0}^{d_{e}-1} \sum_{l=0}^{k} 2 A_{l 0} s \\
+\sum_{k=d_{e}}^{K} \ln \left(\cosh \left(\sum_{l=k_{e}}^{K} X_{l 0} s\right)\right)+\sum_{k=d_{e}}^{K} \ln \left(\cosh \left(\sum_{l=k_{e}}^{K} Y_{l 0} s\right)\right) \\
+\sum_{k=K^{+}+1}^{K} \ln \left(\cosh \left(\sum_{l=k_{e}}^{k} X_{l n} s\right)\right)+\sum_{k=K+1}^{d_{e}+K-1} \ln \left(\cosh \left(\sum_{l=k_{e}}^{k} Y_{l n} s\right)\right) \\
+\phi_{1 e}(s)
\end{array}\right. \\
& \text { where } k_{e}=k-\left(d_{e}-1\right), \quad d_{e}=\frac{d}{2} \quad \text { and } d \text { is even. }
\end{aligned}
$$


For $d$ odd and with $O_{1 o}(s)$ defined as

$$
\begin{aligned}
& \phi_{10}(s)=\frac{d \mathrm{~V}_{0} s^{2}}{4} \operatorname{sum}\left(|\mathbf{W}|^{2}\right)-\ln (s)
\end{aligned}
$$

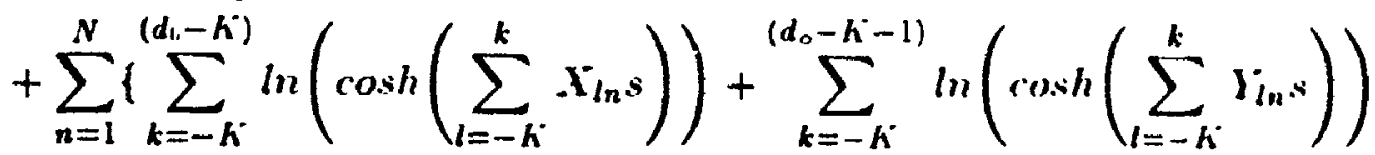

$$
\begin{aligned}
& +\sum_{k=d_{o}-k+1}^{K} \ln \left(\cosh \left(\sum_{l=k-d_{o}}^{k} \boldsymbol{X}_{l n} s\right)\right)+\sum_{k=t, k, k}^{k} \ln \left(\cosh \left(\sum_{l=k-\left(d_{s}-1\right)}^{k} I_{l n} \cdot s\right)\right) \\
& \left.+\sum_{k=K^{+}+1}^{\left(d_{0}+K^{*}\right)} \ln \left(\cosh \left(\sum_{l=k-d_{0}}^{h} X_{l n} s\right)\right)+\sum_{k=K^{+}+1}^{\left(d_{o}+k-1\right)} \ln \left(\cosh \left(\sum_{l=k-\left(l_{n},-l\right)}^{K} I_{l n} \cdot s\right)\right)\right\}
\end{aligned}
$$

we have

$$
\begin{aligned}
& \left(-\sum_{k=0}^{K} \sum_{l=0}^{k} 2 A_{10} s-\sum_{k=K^{+}+1}^{d_{0}-1} \sum_{l=0}^{K} 2 A_{l 0} s\right.
\end{aligned}
$$

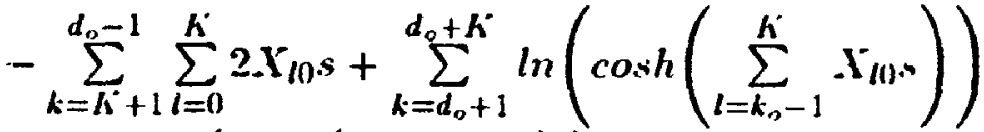

$$
\begin{aligned}
& +\sum_{k=d_{0}+1}^{d_{o}+K^{\circ}} \ln \left(\cosh \left(\sum_{l=k_{0}-1}^{K} Y_{l 0^{s}}\right)\right)+o_{10}(s) \quad ; \text { for } d_{b}>K^{-} \\
& -\sum_{k=0}^{d_{o}-1} \sum_{l=0}^{k} 2 A_{l 0} s-\sum_{k=0}^{d_{o}-1} \sum_{l=0}^{d_{o}} 2 X_{l 0} s \\
& \phi(s)=\left\{+\sum_{k=0}^{d_{o}-1} \ln \left(\cosh \left(\sum_{l=1}^{d_{o}} Y_{l 0} s\right)\right)+\sum_{k=d_{o}+1}^{2 d_{o}} \ln \left(\cosh \left(\sum_{l=k_{n}-1}^{d_{n}} X_{l 0} s\right)\right)\right. \\
& +\sum_{k=d_{o}+1}^{2 d_{o}-1} \ln \left(\cosh \left(\sum_{l=k_{o}}^{d_{o}} Y_{l \Omega} s\right)\right)+\phi_{1 o}(s) \quad \text { for } d_{o}=K \\
& -\sum_{k=0}^{d_{o}-1} \sum_{l=0}^{k} 2 A_{l 0} s-\sum_{k=0}^{d_{o}-1} \sum_{l=0}^{d_{o}} 2 X_{(1)} s \\
& +\sum_{k=d_{0}+1}^{K} \ln \left(\cosh \left(\sum_{l=h_{0}-1}^{k} X_{l 0} s\right)\right)+\sum_{k=K^{\prime}+1}^{d_{0}+k^{-}} \ln \left(\cosh \left(\sum_{l=k_{0,1}-1}^{K} X_{l 0} \cdot s\right)\right) \\
& +\sum_{k=d_{0}}^{K} \ln \left(\cosh \left(\sum_{l=k_{0}}^{k} Y_{l n} s\right)\right)+\sum_{k=K+1}^{d_{0}+K-1} \ln \left(\cosh \left(\sum_{l=k_{0}}^{K} Y_{l !} s\right)\right) \\
& \left(+\phi_{10}(s) \quad \text { :for } d_{o}<\boldsymbol{K}\right. \\
& \text { where } k_{o}=k-\left(d_{o}-1\right), \quad d_{o}=\frac{(d-1)}{2} \text { and } d \text { is orld. }
\end{aligned}
$$


The first derivative of $\phi(s)$ is then expressed as follows. For $d$ even and with

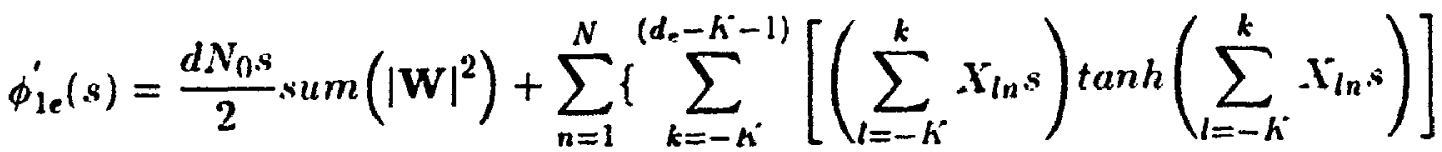

$$
\begin{aligned}
& +\sum_{k=-K^{\prime}}^{\left(d_{e}-K^{-1}\right)}\left[\left(\sum_{l=-K^{\prime}}^{k} Y_{l n} s\right) \tanh \left(\sum_{l=-K^{\prime}}^{k} Y_{l n} s\right)\right] \\
& +\sum_{k=d_{e}-K}^{K}\left[\left(\sum_{l=k-\left(d_{e}-1\right)}^{k} X_{l n} s\right) \tanh \left(\sum_{l=k-\left(d_{e}-1\right)}^{k} X_{l n} s\right)\right] \\
& +\sum_{k=d_{e}-K^{\prime}}^{K}\left[\left(\sum_{l=k-\left(d_{e}-1\right)}^{k} Y_{l n} s\right) \tanh \left(\sum_{l=k-\left(d_{e}-1\right)}^{k} Y_{l n} s\right)\right] \\
& +\sum_{k=K^{\prime}+1}^{\left(d_{e}+K-1\right)}\left[\left(\sum_{l=k-\left(d_{e}-1\right)}^{K} X_{l n} s\right) \tanh \left(\sum_{l=k-\left(d_{e}-1\right)}^{K} X_{l n} s\right)\right] \\
& \left.+\sum_{k=K+1}^{\left(d_{e}+K-1\right)}\left[\left(\sum_{l=k-\left(d_{e}-1\right)}^{K} Y_{l n} s\right) \tanh \left(\sum_{l=k-\left(d_{e}-1\right)}^{K} Y_{l n} s\right)\right]\right\}-s^{-1}
\end{aligned}
$$

we obtain

$$
\begin{aligned}
& \phi^{\prime}(s)= \\
& \left(-\sum_{k=0}^{K} \sum_{l=0}^{k} 2 A_{l 0}-\sum_{k=k^{\prime}+1}^{\left(d_{e}-1\right)} \sum_{l=0}^{K} 2 A_{l 0}+\sum_{k=d_{e}}^{\left(d_{e}+K-1 !\right.}\left[\left(\sum_{l=k_{e}}^{K} X_{l 0} s\right) \tanh \left(\sum_{l=k_{e}}^{K} X_{l 0} s\right)\right]\right. \\
& +\sum_{k=d_{e}}^{\left(d_{e}+K-1\right)}\left[\left(\sum_{l=k_{e}}^{K} Y_{l 0} s\right) \tanh \left(\sum_{l=k_{e}}^{K} Y_{l 0} s\right)\right]+\phi_{1 e}^{\prime}(s) \quad \text { for } d_{e}>K \\
& -\sum_{k=0}^{d_{e}-l} \sum_{l=0}^{k} 2 A_{l 0}+\sum_{k=d_{e}}^{K}\left[\left(\sum_{l=k_{e}}^{K} X_{l 0} s\right) \tanh \left(\sum_{l=k_{e}}^{K} X_{l 0} s\right)\right] \\
& +\sum_{k=d_{e}}^{K}\left[\left(\sum_{l=k_{e}}^{h} Y_{i n} s\right) \tanh \left(\sum_{l=k_{e}}^{K} Y_{l 0} s\right)\right]+\sum_{k=K^{\prime}+1}^{d_{e}+K-1}\left[\left(\sum_{l=k_{e}}^{k} X_{l 0} s\right) \tanh \left(\sum_{l=k_{e}}^{k} X_{l 0} s\right)\right] \\
& +\sum_{k=K^{\prime}+1}^{d_{e}+K-1}\left[\left(\sum_{l=k_{e}}^{k} Y_{l 0} s\right) \tanh \left(\sum_{l=k_{e}}^{k} Y_{l 0} s\right)\right]+\phi_{1 e}^{\prime}(s) \quad \text {; for } d_{e} \leq K \\
& \text { where } k_{e}=k-\left(d_{e}-1\right), d_{e}=\frac{d}{2} \text { and } d \text { is even. }
\end{aligned}
$$


For $d$ odd and with

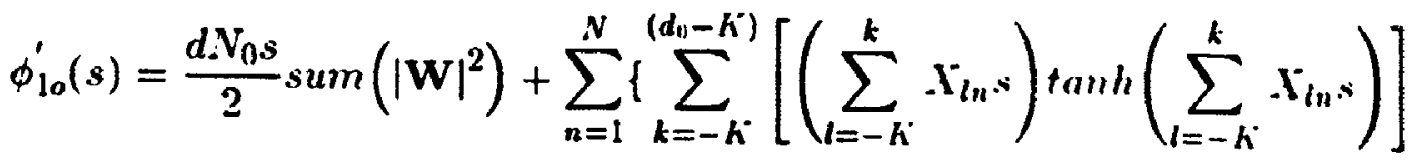

$$
\begin{aligned}
& +\sum_{k=-h^{-}}^{\left(d_{o}-h^{-1)}\right.}\left[\left(\sum_{l=-h^{-}}^{k} Y_{l n} s\right) \tanh \left(\sum_{l=-h^{-}}^{k} \xi_{i n} s\right)\right] \\
& +\sum_{k=d_{0}-k+1}^{k}\left[\left(\sum_{l=k-d_{0}}^{k} X_{l n} s\right) \tanh \left(\sum_{l=k-d_{0}}^{k} X_{l n} s\right)\right] \\
& +\sum_{k=d_{0}-K}^{K}\left[\left(\sum_{l=k-\left(d_{0}-1\right)}^{k} Y_{l n} \cdot s\right) \tanh \left(\sum_{l=k-\left(d_{0}-1\right)}^{k} Y_{l n} \cdot s\right)\right] \\
& +\sum_{k=K+1}^{\left(d_{o}+k\right)}\left[\left(\sum_{l=k-d_{0}}^{h} X_{l n} s\right) \tanh \left(\sum_{l=k-d_{0}}^{K} X_{l n} s\right)\right] \\
& \left.+\sum_{k=k+1}^{\left(d_{0}+k-1\right)}\left[\left(\sum_{l=k-\left(d_{0}-1\right)}^{h} Y_{l n} s\right) \tanh \left(\sum_{l=k-\left(d_{o}-1\right)}^{h} Y_{l n} \cdot s\right)\right]\right\}-.^{-1}
\end{aligned}
$$

we have

$$
\begin{aligned}
& \left(-\sum_{k=0}^{K} \sum_{l=0}^{k} 2 A_{l 0}-\sum_{k=K^{j}+1}^{d_{0}-1} \sum_{l=0}^{K} 2 A_{l 0}-\sum_{k=K^{k}+1}^{d_{0}-1} \sum_{l=0}^{K} 2 Y_{l 0}\right. \\
& +\sum_{k=d_{0}+1}^{d_{0}+H^{*}}\left[\left(\sum_{l=k_{0}-1}^{h} X_{l n} s\right) \tanh \left(\sum_{l=k_{0}-1}^{h} X_{l 0} *\right)\right]
\end{aligned}
$$

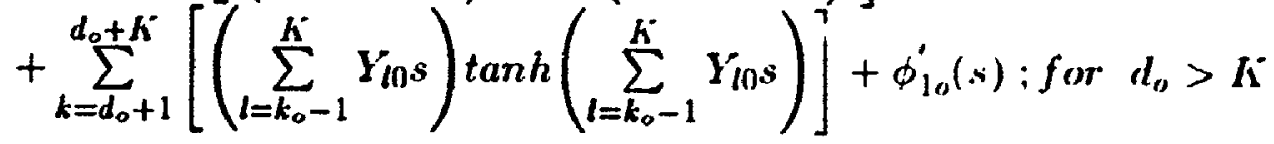

$$
\begin{aligned}
& \phi^{\prime}(s)=\{ \\
& -\sum_{k=0}^{d_{0}-1} \sum_{l=0}^{k} 2 A_{l 1}-\sum_{k=0}^{d_{0}-1} \sum_{l=0}^{d_{0}} 2 X_{l 0}+\sum_{k=0}^{d_{0}-1}\left[\left(\sum_{l=1}^{d_{0}} Y_{l(1) \cdot 5}\right) \tanh \left(\sum_{l=1}^{d_{l i}} Y_{l(1, i)}\right)\right] \\
& +\sum_{k=d_{o}+1}^{2 d_{0}}\left[\left(\sum_{l=k_{o}-1}^{d_{o}} X_{l 0} s\right) \tanh \left(\sum_{l=k_{o}-1}^{d_{o}} X_{l 0} s\right)\right] \\
& +\sum_{k=d_{o}+1}^{2 d_{o}-1}\left[\left(\sum_{l=k_{0}}^{d_{0}} Y_{l n} s\right) \tanh \left(\sum_{l=k_{0}}^{d_{o}} Y_{l n} s\right)\right]+\phi_{1 v}^{\prime}(s) \quad: \text { for } d_{o}=\boldsymbol{K} \\
& \text { where } k_{o}=k-\left(d_{o}-1\right), \quad d_{o}=\frac{(d-1)}{2} \quad \text { ared } \quad d \text { is odd. }
\end{aligned}
$$


and

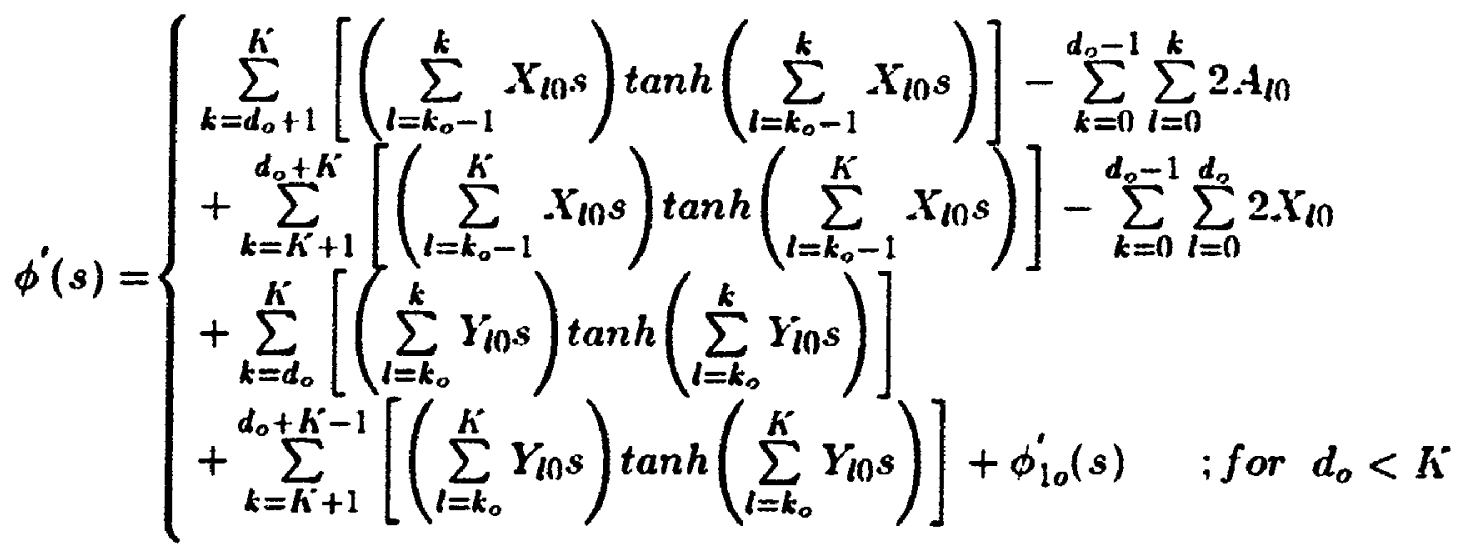

$$
\begin{aligned}
& \text { where } k_{0}=k-\left(d_{0}-1\right), d_{0}=\frac{(d-1)}{2} \text { and } d \text { is odd. }
\end{aligned}
$$

Similarly, the second derivative of $\phi(s)$ is expressed as follows. For $d$ even and with

$$
\begin{aligned}
& \phi_{1 e}^{\prime \prime}(s)=\frac{d N_{0}}{2} s u m\left(|W|^{2}\right)+s^{-2} \\
& +\sum_{n=1}^{N}\left\{\sum_{k=-K}^{\left(d_{e}-k-1\right)}\left[\left(\sum_{l=-K}^{k} X_{l n} s\right)^{2} \operatorname{sech}^{2}\left(\sum_{l=-K}^{k} X_{l n} s\right)\right]\right. \\
& +\sum_{k=-K}^{\left(d_{e}-K-1\right)}\left[\left(\sum_{l=-K}^{k} Y_{l n} s\right)^{2} \operatorname{sech}^{2}\left(\sum_{l=-K}^{k} Y_{l n} s\right)\right] \\
& +\sum_{k=d_{e}-K}^{K}\left[\left(\sum_{l=k-\left(d_{e}-1\right)}^{k} X_{l n} s\right)^{2} \operatorname{sech}^{2}\left(\sum_{l=k-\left(d_{e}-1\right)}^{k} X_{l n} s\right)\right] \\
& +\sum_{k=d_{e}-k}^{k}\left[\left(\sum_{l=k-\left(d_{e}-1\right)}^{k} Y_{l n} s\right)^{2} \operatorname{sech}^{2}\left(\sum_{l=k-\left(d_{e}-1\right)}^{k} Y_{l n} s\right)\right] \\
& +\sum_{k=K+1}^{\left(d_{e}+K-1\right)}\left[\left(\sum_{l=k-\left(d_{e}-1\right)}^{K} X_{l n} s\right)^{2} \operatorname{sech}^{2}\left(\sum_{l=k-\left(d_{e}-1\right)}^{K} X_{l n} s\right)\right] \\
& \left.+\sum_{k=k^{\prime}+1}^{\left(d_{e}+K-1\right)}\left[\left(\sum_{l=k-\left(d_{e}-1\right)}^{K} Y_{l n} s\right)^{2} \operatorname{sech}^{2}\left(\sum_{l=k-\left(d_{e}-1\right)}^{K} Y_{l n} s\right)\right]\right\}
\end{aligned}
$$

we obtain 


$$
\begin{aligned}
& \phi^{\prime \prime}(s)=
\end{aligned}
$$

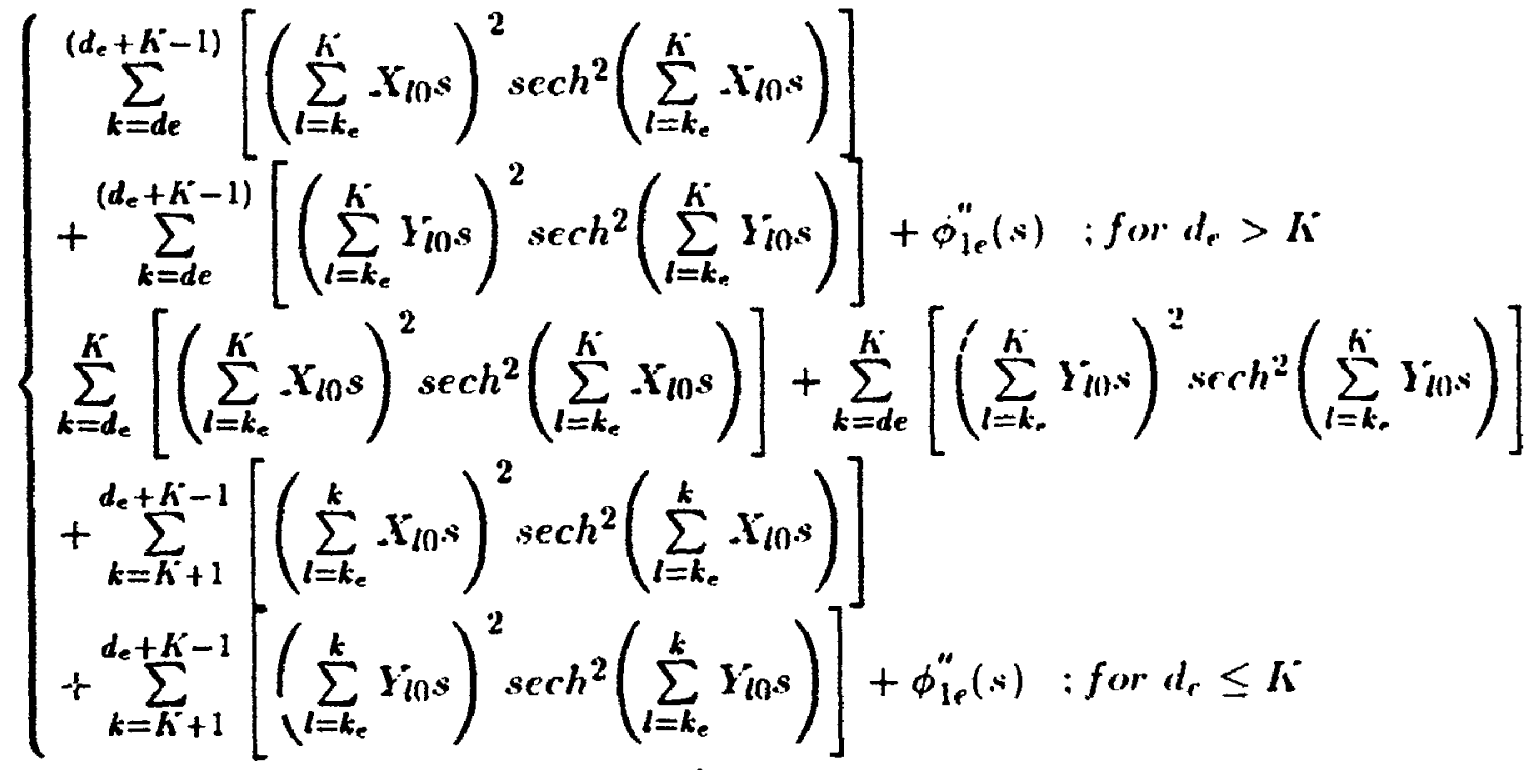

$$
\begin{aligned}
& \text { where } k_{e}=k-\left(d_{e}-1\right), \quad d_{e}=\frac{d}{2} \text { and } d \text { is cinn. }
\end{aligned}
$$

For $d$ odd and with

$$
\begin{aligned}
& \phi_{10}^{\prime \prime}(s)=\frac{d N_{0}}{2} \operatorname{sum}\left(|\mathrm{W}|^{2}\right)+\sum_{n=1}^{N}\left(\sum_{k=-k}^{\left(d_{n}-k\right)}\left[\left(\sum_{l=-k}^{k} X_{l n} \cdot s\right)^{2} \operatorname{sech} 2\left(\sum_{l=-k}^{k} X_{l n} \cdot s\right)\right]\right. \\
& +\sum_{k=-K^{k}}^{\left(d_{o}-K^{-1}\right)}\left[\left(\sum_{l=-K^{K}}^{k} Y_{l n} s\right)^{2} \operatorname{sech}^{2}\left(\sum_{l=-K^{K}}^{k} Y_{l n} s\right)\right] \\
& +\sum_{k=d_{0}-k+1}^{k}\left[\left(\sum_{l=k-d_{0}}^{k} \boldsymbol{X}_{l n} s\right)^{2} \operatorname{sech}^{2}\left(\sum_{l=k-d_{0}}^{k} X_{l n} s\right)\right] \\
& +\sum_{k=d_{o}-K}^{K}\left[\left(\sum_{l=k-\left(d_{0}-1\right)}^{k} Y_{i n} s\right)^{2} \operatorname{sech}^{2}\left(\sum_{l=k-\left(d_{o}-1\right)}^{k} Y_{l n} \cdot s\right)\right] \\
& +\sum_{k=k+1}^{\left(d_{o}+k\right)}\left[\left(\sum_{l=k-d_{o}}^{k} \mathbf{X}_{l n} s\right)^{2} \operatorname{sech}^{2}\left(\sum_{l=k-d_{o}}^{K} X_{l n} s\right)\right] \\
& \left.+\sum_{k=K+1}^{\left(d_{o}+K-1\right)}\left[\left(\sum_{l=k-\left(d_{0}-1\right)}^{K} Y_{l n} s\right)^{2} \operatorname{sech}^{2}\left(\sum_{l=k-\left(d_{0}-1\right)}^{K} Y_{l u} s\right)\right]\right\}+s^{-2}
\end{aligned}
$$


we then have

$$
\begin{aligned}
& \phi^{\prime \prime}(s)= \\
& \left\{\begin{array}{l}
\sum_{k=d_{0}+1}^{d_{n+K}}\left[\left(\sum_{l=k_{0}-1}^{K} X_{l n} s\right)^{2} \operatorname{sech}^{2}\left(\sum_{l=k_{0}-1}^{K} X_{l 0} s\right)\right] \\
+\sum_{k=d_{o}+1}^{d_{o}+K}\left[\left(\sum_{l=k_{0}-1}^{K} Y_{i n} s\right)^{2} \operatorname{sech}^{2}\left(\sum_{l=k_{0}-1}^{K} Y_{l n} s\right)\right]+\phi_{l o}^{\prime \prime}(s) \quad ; \text { for } d_{o}>K^{*}
\end{array}\right. \\
& \sum_{k=0}^{d_{0}-1}\left[\left(\sum_{l=1}^{d_{0}} Y_{i n} s\right)^{2} \operatorname{sech}^{2}\left(\sum_{l=1}^{d_{0}} Y_{l n} s\right)\right] \\
& +\sum_{k=d_{0}+1}^{2 d_{0}}\left[\left(\sum_{l=k_{0}-1}^{d_{0}} \boldsymbol{X}_{l n} s\right)^{2} \operatorname{sech}^{2}\left(\sum_{l=k_{0}-1}^{d_{0}} X_{l 0} s\right)\right] \\
& \left\{+\sum_{k=d_{o}+1}^{2 d_{o}-1}\left[\left(\sum_{l=k_{o}}^{d_{o}} Y_{l n} s\right)^{2} \operatorname{sech}^{2}\left(\sum_{l=k_{o}}^{d_{o}} Y_{l n} s\right)\right]+\phi_{10}^{\prime \prime}(s) \quad ; \text { for } d_{o}=K\right. \\
& \sum_{k=d_{0}+1}^{h}\left[\left(\sum_{l=k_{0}-1}^{k} X_{10} s\right)^{2} \operatorname{sech}^{2}\left(\sum_{l=k_{0}-1}^{k} X_{10} s\right)\right] \\
& +\sum_{k=K^{j}+1}^{d_{o}+K}\left[\left(\sum_{l=k_{o}-1}^{K} X_{l 0} s\right)^{2} \operatorname{sech}^{2}\left(\sum_{l=k_{o}-1}^{K} X_{l 0} s\right)\right] \\
& +\sum_{k=d_{o}}^{K}\left[\left(\sum_{l=k_{o}}^{k} Y_{l n} s\right)^{2} \operatorname{sech}^{2}\left(\sum_{l=k_{o}}^{k} Y_{l 0} s\right)\right] \\
& +\sum_{k=h^{\prime}+1}^{d_{0}+K^{K}-1}\left[\left(\sum_{l=k_{0}}^{K} Y_{l 0^{s}}\right)^{2} \operatorname{sech}^{2}\left(\sum_{l=k_{0}}^{K} Y_{l 0^{s}}\right)\right]+\phi_{10}^{\prime}(s) \quad \text {;for } d_{o}<K^{\prime \prime}
\end{aligned}
$$

where $k_{o}=k-\left(a_{c}-1\right), d_{o}=\frac{(d-1)}{2}$ and $d$ is odd.

Two different expressions are also obtained for the saddlepoint search starting point so depending on whether $d$ is odd or even. As done previously, the first step involves replacing the interference terms in equations 5.49 ( $d$ even) and 5.50 ( $d$ odd) by gaussian 
noise of equal variance. Referring to these equations and noticing that their respective first terms can be split into, for $d$ even,

and, for $d$ odd,

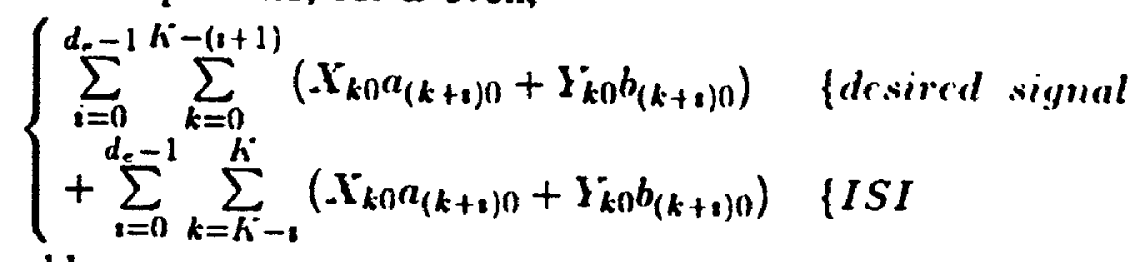

$$
\left\{\begin{array}{lll}
\sum_{i=0}^{d_{o}} \sum_{k=0}^{K-(i+1)} X_{k=0} a_{(k+i) 0}+\sum_{i=0}^{d_{o}-1} \sum_{k=0}^{K-(i+1)} Y_{k 0} b_{(k+1) 0} & \text { \{desired sigmal } \\
+\sum_{i=0}^{d_{o}} \sum_{k=K^{-}-i}^{K} X_{k 0} a_{(k+i) 0}+\sum_{i=0}^{d_{0}-1} \sum_{k=K_{-1}^{K}}^{K} Y_{k 0} b_{(k+1) 0} & \text { \{ISI }
\end{array}\right.
$$

one obtains a total gaussian variance of

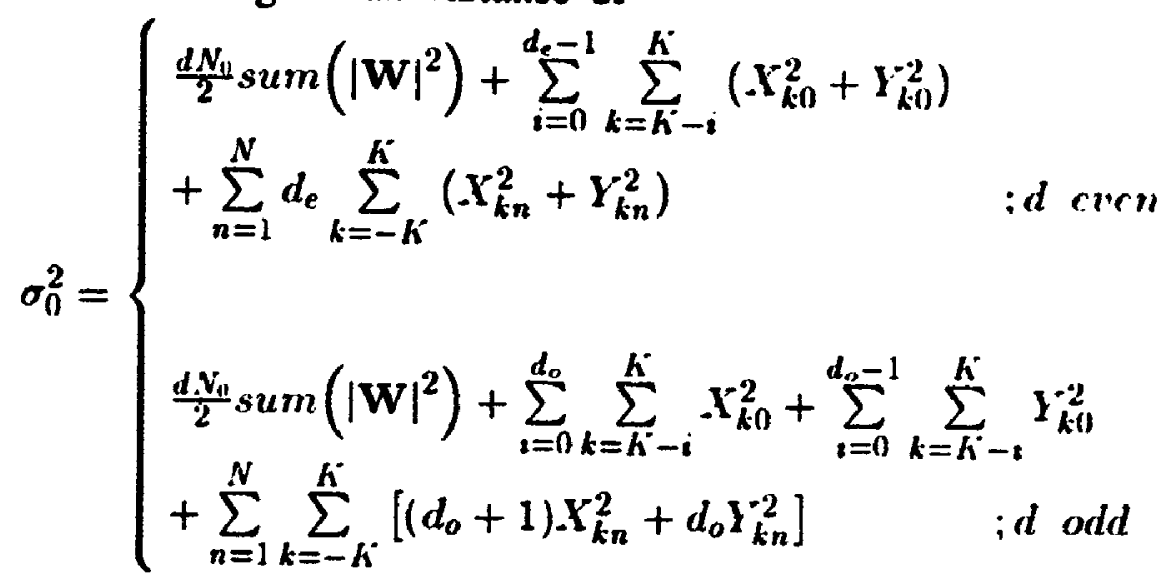

The first terms in $\sigma_{0}^{2}$ have been determined by noting that $\operatorname{Rr}\left(\mathbf{W}^{*} \mathbf{T} \mathbf{V}_{\mathbf{i}}\right)$ and $\operatorname{Im}\left(\mathbf{W}^{* \mathbf{T}} \mathbf{V}_{\mathbf{i}}\right)$ have variances of $\frac{N_{0}}{2} \operatorname{sum}\left(|\mathbf{W}|^{2}\right)$ and that $d=2 d_{e}$ and $d=2 d_{o}+1$.

The saddlepoint search starting point is therefore

$$
s_{0}= \begin{cases}\frac{1}{2 \sigma_{0}^{2}}\left\{\sum_{i=0}^{d_{e}-1} \sum_{k=0}^{K}\left(X_{k 0}+Y_{k 0}\right)\right. & ; d \text { cren } \\ \left.+\left[\left(\sum_{i=0}^{d_{e}-1} \sum_{k=0}^{K-(i+1)}\left(X_{k 0}+Y_{k 0}\right)\right)^{2}+4 \sigma_{0}^{2}\right]^{\frac{1}{2}}\right\} & \\ \frac{1}{2 \sigma_{0}^{2}}\left\{\sum_{i=0}^{d_{o}} \sum_{k=0}^{K-(i+1)} X_{k 0}+\sum_{i=0}^{d_{o}-1} \sum_{k=0}^{K-(i+1)} Y_{k 0}\right. & \\ \left.+\left[\left(\sum_{i=0}^{d_{o}} \sum_{k=0}^{K-(i+1)} X_{k 0}+\sum_{i=0}^{d_{o}-1} \sum_{k=0}^{K-(i+1)} Y_{k 0}\right)^{2}+4 \sigma_{0}^{2}\right]^{\frac{1}{2}}\right] ; d \text { odd }\end{cases}
$$




\subsection{Summary of analytical assumptions}

Prior to presenting and discussing the results obtained with this semi-analytical technique, it is worthwhile to summarize the underlying assumptions. It is seen that four of the six assumptions inherent in the purely analytical approach of Chapter 3 (Section 3.6) have been eliminated. Two remain, namely

1. Feedback decisions are assumed correct.

2. The Viterbi decoder path memory is assumed infinite.

The first assumption will tend to make results optimistic at low values of $\frac{E_{6}}{N_{10}}$ and $S$ while the second has a negligible influence if the decoder memory is equal to at least five times the constraint length [154]. A single additional assumption has been introduced i.e.

3. In the coded case with ao interleaving, it is assumed that the $d$ bits that differ between the two paths under consideration for a given pairwise error probability $P_{2}(d)$ are successive bits.

As discussed earlier, this assumption is all the more realistic given the quasi-static nature of the channel and its influence is small for $d$ equal to or only slightly larger than $d_{\text {free }}$ where $P_{2}(d)$ is largest.

\subsection{Results}

The results in this Chapter are based not only on the nine-tap channel model with exponential delay profile and fixed tap spacing of half the rms delay spread discussed in Chapter 4 but also (and mostly) on the "cluster" channel model developed as a result of measurements [60] at $1.5 \mathrm{GHz}$ and briefly discussed in Chapter 2 . This model involves representing the channel's low-pass equivalent impulse response by [60]

$$
h(t)=\sum_{l=0}^{\infty} \sum_{k=0}^{\infty} \beta_{k l} \delta\left(t-T_{l}-\tau_{k l}\right) \exp \left(j \theta_{k l}\right)
$$

where the phase $\theta_{k l}$ is Lniformly distributed from $-\pi$ to $\pi$ and where the gain $\beta_{k l}$ of the $k^{\text {th }}$ ray within the $l^{\text {th }}$ cluster is Rayleigh distributed with mean-square value $\overline{\beta_{k l}^{2}}$, 
i.e. $[60]$

$$
p\left(\beta_{k l}\right)=\frac{2 \beta_{k l}}{\overline{\beta_{k l}^{2}}} \exp \left(-\frac{\beta_{k l}^{2}}{\beta_{k l}^{2}}\right)
$$

The $\overline{\beta_{k l}^{2}}$ 's decrease exponentially as a function of the cluster power-delay time constant $\Gamma$ and the ray power-delay time constant $\gamma[60]$

$$
\overline{\beta_{k l}^{2}}=\overline{\beta_{00}^{2}} \exp \left(-\frac{T_{l}}{\Gamma}\right) \exp \left(-\frac{T_{k l}}{\gamma}\right)
$$

The arrival times of the clusters $T_{l}$ and of the rays $\tau_{k l}$ within the clusters are represented by two Poisson processes with different but fixed rates, $\Lambda$ and $\lambda$ respectively, i.e. [60]

$$
p\left(T_{l} \mid T_{1-1}\right)=\Lambda \exp \left(-\Lambda\left(T_{l}-T_{l-1}\right)\right) \text { for } l>0
$$

and

$$
p\left(\tau_{k l} \mid \tau_{(k-1) l}\right)=\lambda \exp \left(-\lambda\left(\tau_{k l}-\tau_{(k-1) l}\right)\right) \text { for } k, l: 0
$$

This cluster model was found to give a good fit to measurements on the first floor of a two-story medium-size office building when the four parameters describing the model were set to the following values [60]

1. Cluster mean interarrival time: $\frac{1}{\Lambda}=200 \mathrm{~ns}$ to $300 \mathrm{~ns}$.

2. Ray mean interarrival time: $\frac{1}{\lambda}=5 \mathrm{~ns}$.

3. Cluster power-delay time constant: $\Gamma=60 \mathrm{~ns}$.

4. Ray power-delay time constant: $\gamma=20 \mathrm{~ns}$.

The range given for the first parameter results from Saleh et al.'s empirical observations which only indicate that the cluster arrival rate "needs to be roughly in the range of $\frac{1}{2(11) \mathrm{ns}}$ to $\frac{1}{30 \mathrm{~ns}}$ " [60] in order to obtain a good fit to their measurements. The total multipath power gain $\sum_{l} \sum_{k} \overline{\beta_{k l}^{2}}$ was normalized to unity as was done with the simple nine-tap channel model ${ }^{62}$ in Chapter 4. Five clusters of one hundred rays were generated for each impulse response realization. This implies that clusters typically overlap as each cluster has an average duration of $5 \times 100 \mathrm{~ns}=500 \mathrm{~ns}$. The total average length of the 62 Recall from Chapter 4 that the nine-tap model's total multipath power gain is actually 0.98 . 
impulse response is then $1300 \mathrm{~ns}$ to $1700 \mathrm{~ns}$, depending on whether $\frac{1}{\lambda}$ is set to $200 \mathrm{~ns}$ or 300 ns. It should however be noted that many of these 500 rays are very weak as the second and third clusters' average power gains are down to respectively $3 \%$ and $0.1 \%$ of the first cluster's power gain. In an average sense, only the first two clusters thus contribute rays which have at least $1 \%$ of the most powerful ray's power which tends to limit the effective average duration of the impulse response to the $300 \mathrm{~ns}$ to 400 ns range. In fact, using more than five clusters was found to have a negligible effect on the rms delay spread $\sigma_{d}$ which was computed for all 10000 independent sets of portable-to-base station antenna impulse response realizations according to ${ }^{63}$

$$
\sigma_{d}=\sqrt{\overline{\tau^{2}}-(\bar{\tau})^{2}}
$$

where

$$
\overline{\tau^{n}}=\frac{\sum_{k} \tau_{k}^{n} \beta_{k}^{2}}{\sum_{k} \beta_{k}^{2}} \quad n=1,2
$$

Figure 5.1 shows the resulting cumulative distribution functions of $\mathrm{ms}$ delay spread for mean cluster interarrival times of $200 \mathrm{~ns}$ and $300 \mathrm{~ns}$. With $\frac{1}{\lambda}=200 \mathrm{~ns}$, the average ms delay spread was found to be $45.5 \mathrm{~ns}$, the median ${ }^{64} \mathrm{~ms}$ delay spread $45.0 \mathrm{~ns}$, and the worst-case $\mathrm{mms}$ delay spread $100.7 \mathrm{~ns}$. On the other hand, with $\frac{1}{\lambda}=300 \mathrm{~ns}$, the average $\mathrm{ms}$ delay spread was found to be $40.0 \mathrm{~ns}$, the median $\mathrm{ms}$ delay spread $38.5 \mathrm{~ns}$, and the worst-case rms delay spread $97.1 \mathrm{~ns}$. These values are thus much larger than the measured median rms delay spread of $25 \mathrm{~ns}$ quoted in [60]. The difference results essentially from the fact that Saleh et al. [60] were only able to measure those paths suffering less than a $100 \mathrm{~dB}$ path loss with respect to the transmitted power. Whenever the most powerful received ray's path loss approached $100 \mathrm{~dB}$, slightly weaker rays, which could have significantly contributed to increasing the rms delay spread, were most probably truncated. In fact, other measurements within the first floor of the same building where only those rays suffering more than a $140 \mathrm{~dB}$ path loss were truncated

63 This represents the rms delay spread expression for a discrete channel model. The corresponding expression for a continuous model was given in Chapter 2 by equations 2.8 and 2.9 .

64 Note that the distinction between median and mean ms delay spreads is often disregarded as they rarely differ by more than 1 or 2 ns. 
yielded a median rms delay spread of approximately $35 \mathrm{~ns}$ [87, p.321]. In fitting the model to the measurements, Saleh $e t a l$. included the effect of path loss and truncated those simulated paths attenuated by more than $100 \mathrm{~dB}$ with respect to transmitted power thus yielding the $25 \mathrm{~ns}$ median rms delay spread. On the other hand, the simulation performed in this work normalized total multipath gain to unity and included all 500 rays in the rms delay spread calculation. In fact, the $38.5 \mathrm{~ns}$ median rms delay spread obtained with $\Lambda=\frac{1}{300 n s}$ tends to corroborate the results of a recent independent study [151] with this model where an median rms delay spread of $37.9 \mathrm{~ns}$ was also obtained with $\Lambda=\frac{1}{300 \mathrm{~ns}}$ over 2000 impulse response realizations where those paths $60 \mathrm{~dB}$ or more below the most powerful path were truncated. It should also be noted that an increase in the mean cluster interarrival time results in a decrease of the mean rms delay spread; as successive clusters are then subjected to a greater exponential power loss, the rms delay spread decreases as it is calculated by averaging gain-weighted time delays. In order to perform a worst-case evaluation, all performance curves in this work are thus generated with a mean cluster interarrival time of $200 \mathrm{~ns}$.

As complexity is a prime concern for the portable application, the number of forward filter taps $L$ is set to 3 which $^{65}$ was found in Chapter 3 to be nearly optimal, assuming a fixed receiver sampling phase, for a rms delay spread on the order of a quarter to a half of a symbol interval. Many of the results obtained with the cluster model are thus generated with parameters normalized to a signaling rate and thus data rate, assuming uncoded BPSK or rate $1 / 2$ coded QPSK ${ }^{66}$, of $10 \mathrm{Mbits} / \mathrm{s}$ which corresponds to a normalized rms delay spread of $0.45 \mathrm{~T}$. In this case, the symbol interval (and equalizer span) of $100 \mathrm{~ns}$ is almost equal to the worst-case rms delay spread of $100.7 \mathrm{~ns}$. It is at this data rate of $10 \mathrm{Mbits} / \mathrm{s}$ that coding is considered in Section 5.4.1.1 as an alternative to a third space diversity branch with different levels of cochannel interference. As opposed to Chapters 3 and 4 where interferer timing phase with respect

$65 \quad L$ is set to 3 with $L I=2$ and $L 2=0$, i.e. precursor taps, one central tap and no postcursor taps in the forward filters.

66 As in Chapters 3 and 4, rate 1/2 coded QPSK is mostly compared to uncoded BPSK thus maintaining the same rate of information throughput and the same bandwidth. Rate 1/2 coded QPSK will also be compared with uncoded QPSK for a $10 \mathrm{Mbits} / \mathrm{s}$ data rate, i.e. where the coded and uncoded normalized rms delay spreads differ and thus where bandwidth expansion must be taken into account. 
to the desired signal is assumed fixed for all channel realizations, the interferer timing phases are now independent random variables, uniformly distributed from 0 to the symbol interval $T$. The interferers now also have independent random complex signal phases, uniformly distributed between $-\pi$ and $\pi$. The coded results of this Chapter are based on the same convolutional code used in the previous two Chapters, i.e. a rate $1 / 2$, constraint length 3 , free distance 5 code with code generators 5 and 7 . The effect of a lower data rate, i.e. $1 \mathrm{Mbits} / \mathrm{s}$, on the coding vs. space diversity trade-off is then examined in Section 5.4.1.2. The influence of different channel models (nine-tap with fixed spacing vs. cluster) and different interference models (non-fading gaussian vs. fading, non-gaussian) is also the subject of Sections 5.4.2 and 5.4.3 respectively. In these Sections, the receiver sampling phase is assumed fixed to $t_{0}=0.375 T$ which was found in Chapter 3 to be nearly optimal for the exponential delay profile continuous channel model ${ }^{67}$. However, in Section 5.4.4, the influence of continuously optimizing this sampling phase is assessed. All of these results are presented in Figures 5.1 to 5.30 on pages 170-199. Coding gains are expressed, unless stated otherwise, in terms of a $10^{-3}$ average $B E R$ and in terms of a $10^{-2}$ outage rate at a BER of $10^{-3}$. These criteria should provide satisfactory performance for speech communications but other applications such as data will typically require superior BER integrity.

It should initially be noted that the limiting test cases of uncoded BPSK in flat Rayleigh fading with only $L=1$ forward filter tap and no cochannel interference $(\mathrm{N}=0)$ are found in Figure 5.2 to match, for explicit space diversity orders of $M=1$ ana $M=2$, the theoretical prediction ${ }^{68}$ of $[71$, p.474], i.e.

$$
P_{b}=\left(\frac{1-\mu}{2}\right)^{M} \sum_{i=0}^{M-1}\left(\begin{array}{c}
M-1+i \\
i
\end{array}\right)\left(\frac{1-\mu}{2}\right)^{i}
$$

where $\mu=\sqrt{\frac{\left(\frac{E_{0}}{N_{0}}\right)}{\left(1+\frac{\varepsilon_{b}}{N_{0}}\right)}}$. With $M=1$, equation 5.81 simply collapses to the case of uncoded

67 Recall from Chapter 3 that $t_{0}=0.375 T$ was the best sampling phase (out of 8 possible) for the case of no shaping and the second best for the 50\% SRC pulse shape. The best sampling phase could be different however for the cluster model. 
BPSK in flat Rayleigh fading i.e. [71, p.468]

$$
P_{b}=\frac{1-u}{2}
$$

With the only disturbance being thermal noise, which is uncorrelated (even in the shortterm) between the diversity branches, these results correspond to $\mathbf{M}^{\text {th }}$-order maximalratio combining. As the analysis in this work handles the $M$ explicit space diversity branches in the same way that it handles the $L$ implicit diversity branches, i.e. via the $M \times L$-element $q_{k n}$ vectors, these limiting cases with $M^{\text {th }}$-order diversity should also serve to validate the procedure with $L^{\text {th }}$-order implicit diversity. Similarly, the coded BPSK case with ideal interleaving and no diversity (i.e. $M=1$ ) in flat Rayleigh fading is also seen in Figure 5.2 to match the theoretical prediction of [71, section 7.8.3]; with $P_{b}=\sum_{d=d_{\text {free }}}^{\infty} \beta_{d} P_{2}(d)$, the pairwise error probabilities are given by

$$
P_{2}(d)=\left(\frac{1-\mu}{2}\right)^{d} \sum_{k=0}^{d-1}\left(\begin{array}{c}
d-1+k \\
k
\end{array}\right)\left(\frac{1+\mu}{2}\right)^{k}
$$

where $\mu=\sqrt{\frac{\left(\frac{R_{c} E_{b}}{N_{n}}\right)}{\left(1+\frac{R_{c} E_{b}}{N_{0}}\right)}}$. Slight differences between the analytical curves and the semianalytical method of moments results are observed at high $\frac{E_{b}}{N_{11}}$ for the $\mathrm{M}=2$, uncoded and $M=1$, coded cases. At an average BER of $10^{-6}$, these discrepancies are however very small, i.e. less than $0.5 \mathrm{~dB}$, and can simply be attributed to the statistics of the finite set $(10000)$ of channel realiza' is is.

On the other hand, as the open literature contains relatively few results concerning coding with no interleaving, it is somewhat more difficult to use previous results to ascertain the exactness of the semi-analytical method of moments in this case. Lutz [167] has assessed the $\frac{E_{s}}{N_{11}}$ degradation, relative to ideal interleaving, of different levels of partial interleaving over a Rician slowly fading channel for coherent BPSK with both block and convolutional codes incluwing the rate $\frac{1}{2}$, constraint length 3 , free distance 5 code used in this thesis; however, no results are given for the case of no interleaving and all that can be implied is that the degradation in this latter case should be larger than $5 \mathrm{~dB}$. Hanzo et al. [210] have compared the perfurmances of 
a rate $\frac{1}{2}$, constraint length 5 , free distance 7 convolutional code for different degrees of interleaving (including no interleaving) but the scheme involves MSK over a fast Rayleigh fading vehicular mobile radio channel. Krishnamurthi and Gupta [163] have observed large performance degradations for coded coherent BPSK with no interleaving over a slow Rayleigh fading indoor radio channel but their study dealt only with block $(\mathrm{BCH})$ codes and results were stated in terms of word error probability. As discussed in Chapter 2, the most comprehensive set of results dealing with the effects of partial and no interleaving can be found, to the author's knowledge, in Miyagaki et al. [56] but again the study deals strictly with block codes and involves noncoherent FSK over a $20 \mathrm{~Hz}$ fade rate Rayleigh fading channel. More recently, the performance of coherent BPSK with non-interleaved convolutional codes of rate $\frac{1}{2}$ and $\frac{1}{3}$ has been studied [164] for different fade rates over a flat Rayleigh fading channel but for long constraint length codes (i.e. 8 and 9) and for dual-order selection diversity. Hanna et al. [150] have also evaluated, via a bit-by-bit simulation, the performance of coherent QPSK with convolutional coding and no interleaving over a flat Rayleigh fading channel with a fade rate of $B_{d}=6 \mathrm{~Hz}$ and a symbol rate of $\frac{1}{T}=400 \mathrm{ksymbols} / \mathrm{s}$, i.e. $B_{d} T=1.5 \times 10^{-5}$. The results of $[150]$ are based on the same rate $\frac{1}{2}$, constraint length 3 , free distance 5 code used in this thesis; Figure 5.3a thus compares this average BER result with those obtained via the semi-analytical method of moments approach. It is seen that, at a BER of $10^{-3}$, the coded results of [150] are optimistic by $2 \mathrm{~dB}$ with respect to those of this thesis ${ }^{69}$. This discrepancy between the two coded cases, which is nil at low $\frac{E_{b}}{N_{11}}$ but then increases with $\frac{E_{b}}{N_{10}}$, can be essentially atributed to ${ }^{70}$ a small bit error count at high $\frac{E_{b}}{N_{0}}$. The principal author of [150] indicates [211] that a longer simulation run at $\frac{E_{0}}{N_{0}}=24.9 \mathrm{~dB}$ increased the average BER to $8.73 \times 10^{-4}[211]$; this would then reduce the difference, at a BER of $10^{-3}$, to approximately $1 \mathrm{~dB}$. In fact, the results of [164] indicate that when the normalized fade rate falls to 0.0005 , a further drop in fade

69 Note that in flat Rayleigh fading, uncoded BPSK and QPSK perform itentically in terms of average BER as shown in Appendix B. Even with the difference in fade rate (quasi-static vs. $6 \mathrm{~Hz}$ ), the uncoded QrSK results of [150] (with perfect carrier and timing recovery) were too close to be distinguished from the uncoded BPSK curve in Figure 5.3a and are thus not shown.

70 The fact that the QPSK constellation is rotated by $\frac{\pi}{6}$ in [150] could also affect the difference. 
rate will not affect the performance of a non-interleaved ${ }^{71}$ convolutional code. In this sense, the two coded curves in Figure 5.3a should match, i.e. coded, non-interleaved performance in the $6 \mathrm{~Hz}$ fade rate case, which corresponds to a user walking with (hand-held) portable, should be similar to performance in the quasi-static case, e.g. a stationary terminal application. The two would only differ for signaling rates smaller than $\frac{6}{0.0015}=12 \mathrm{ksymbols} / \mathrm{s}$. Hence, for the high signaling rates considered in this thesis, coded, non-interleaved performance will only show an improvement over the quasi-static case for a high speed vehicle which implies, based on the $\mathbf{4 0 0} \mathrm{ksymbols} / \mathrm{s}$ signaling rate of $[211]$, a fade rate of $\left(4 \times 10^{5}\right)(0.0005)=200 \mathrm{~Hz}$. For a $1.8 \mathrm{GHz}$ carrier frequency, this corresponds to a vehicle speed of $\frac{(200 \mathrm{~Hz})\left(3 \times 10^{\mathrm{N}} \frac{\mathrm{m}}{\mathrm{s}}\right)}{1 . \times \times 10^{2} \mathrm{H} z}=120 \mathrm{~km} / \mathrm{hr}$.

A comparison with Figure 5.2 shows that the non-interleaved system produces in this quasi-static situation a degradation of approximately $19 \mathrm{~dB}$ at a BER of $10^{-3}$ with respect to the ideal interleaving case. While the coded, non-interleaved system performs worse, except at high $\frac{E_{2}}{11}$, than its uncoded counterpart in terms of average BER (by $2.2 \mathrm{~dB}$ at a BER of $10^{-3}$ ), Figure 5.3b then shows that the reverse is true in terms of outage rate where a coding gain of $2.4 \mathrm{~dB}$ is observed at an outage rate of $10^{-2}$. When a 50\% SRC pulse shape is used as in Figure 5.4, coding with no interleaving yields a degradation, with respect to the uncoded case, of $3.5 \mathrm{~dB}$ in terms of $10^{-3}$ average $\mathrm{BER}^{72}$ but again produces an improvement of $2.4 \mathrm{~dB}$ at a $10^{-2}$ outage rate. As was observed with the bit-by-bit simulation of Chapter 4, the non-interleaved coded error rates for different static channel realizations are once again far more polarized ${ }^{73}$ than their uncoded counterparts, i.e. the individual coded static channel error rates are either very large or very small. A given coded average BER is thus dominated by fewer channel realizations than a corresponding uncoded average BER of equal value. For a given average BER, this means, as shown in Figure 5.5, that the corresponding

$\pi$ However, performance was found [164] to vary among fade rates smalter than 0.0005 even for small degrees of partial interleaving.

72 In Figure 5.4a, the coded average BER curve does not cross the corresponding uncoded curve as it does in the no shaping case (Figure 5.3a) at $\frac{E_{*}}{N_{0}}=30 \mathrm{~dB}$. However, the coded curve in Figure 5.4a appears to be converging, albeit far more slowly than in Figure 5.3a, towards the uncoded curve.

73 E.g. as a coded system static AWGN channel bit error rate curve has typically a much steeper slope than its uncoded counterpar, a given variation in signal level will thus produce a stronger variation in the coded system's enror rate. 
outage rate at the same value of $\frac{E_{2}}{N_{1}}$ is lower for the coded system than for the uncoded system; e.g. in Figure 5.5a representing the flat fading case with no diversity, at an average BER of $10^{-2}$, the outage rate (for a $10^{-3}$ BER) is found to be $5.2 \times 10^{-2}$ for the coded system and $2.1 \times 10^{-1}$ for the uncoded system. The same holds true at a higher data rate and with diversity: e.g. in Figure 5.5b, at an average BER of $10^{-3}$, the outage rate (for a $10^{-3}$ BER) is found to be $4 \times 10^{-2}$ for the coded system and $9 \times 10^{-2}$ for the uncoded system. Coding is therefore more attractive in terms of outage rate which is really the criterion of interest for portable and stationary terminal applications as it represents the probability that the link is reliable.

\subsubsection{Coding vs. space diversity}

The results in this Section serve as a complement to those of Chapters 3 and 4 in meeting this thesis' main objective which, as specified in Chapter 1 , consists of answering the following question. On a wideband TDMA indoor radio link employing optimum baseband combining with explicit space diversity order 2, is channel coding a viable alternative to increasing the space diversity order to 3 even in the absence of interleaving?

Figure 5.6 displays results obtained with no cochannel interference. Under the assumption of ideal interleaving, the coded two-antenna system yields gains over its uncoded counterpart of $7.7 \mathrm{~dB}$ in terms of average BER and of $11.3 \mathrm{~dB}$ in terms of outage rate. As these gains are still positive with respect to the uncoded threeantenna system, i.e. $4.1 \mathrm{~dB}$ and $7.0 \mathrm{~dB}$ in terms of average BER and outage rate respectively, channel coding would certainly be, as shown with the analysis of Chapter 3, far more desirable than increasing the space diversity order if ideal interleaving could be implemented. With no interleaving, it is then seen that the coded, two-antenna system yields positive coding gains over the corresponding uncoded system of $2.4 \mathrm{~dB}$ in terms of average BER and of $4.4 \mathrm{~dB}$ in terms of outage rate. This represents $73 \%$ of the $3.3 \mathrm{~dB}$ gain in average BER and $100 \%$ of the gain in outage rate obtained by adding a third antenna to the uncoded system. Hence, with only dual space diversity, a short constraint length convolutional code and a data rate of $10 \mathrm{Mbits} / \mathrm{s}$ which makes 
it possible to exploit the channel's implicit diversity effect, the effective diversity order $M^{*} L$ is sufficiently large to alleviate the effects of the absence of interleaving to a point where positive coding gains are still possible. On the other hand. recall from Chapter 2 that positive coding gains with no interleaving have also been observed [56] with explicit space diversity only on a flat fading channel but this typically requires a very large explicit space diversity order $M$ and a very complex code.

\subsubsection{Effect of cochannel interference}

As seen in Figure 5.7, positive coding gains are still achieved in the presence of a single cochannel interferer with a SIR of $10 \mathrm{~dB}$ even with no interleaving. While ideal interleaving enables the two-antenna coded system to outperform its uncoded counterpart by $7.8 \mathrm{~dB}$ in terms of average BER and by $14.6 \mathrm{~dB}$ in terms of outage rate and also outperform the three-antenna uncoded system by $2.8 \mathrm{~dB}$ in terms of average BER and by $7.9 \mathrm{~dB}$ in terms of outage rate, the non-interleaved coded system still yields positive coding gains with respect to the uncoder :wo-antenna system by respectively recuperating $54 \%(2.8 \mathrm{~dB})$ and $80 \%(5.6 \mathrm{~dB})$ of the average BER and outage rate gains obtained by adding a third space diversity branch. The coding gains are therefore mostly larger, in absolute terms, than those obtained with no cochannel interference; however. the coded non-interleaved system now recovers a smaller fraction of the gain obtained with a third diversity branch. By displaying results at $\frac{E_{6}}{N_{10}}=30 \mathrm{~dB}$ i.e. where AWGN power is small relative to the desired signal, Figure 5.8 then shows that these coding gains wil! quickly vanish as the SIR is reduced from this $10 \mathrm{~dB}$ value and become negative when the SIR drops below $8.5 \mathrm{~dB}$. The actual degradation encountered when the SIR drops below $10 \mathrm{~dB}$ is probably even more significant as the assumption of correct feedback decisions then more than likely begins to degrade.

In Figure 5.9, it is then seen that the dual space diversity configuration cannot cancel two equal-power cochannel interferers with a total SIR of $10 \mathrm{~dB}$, thus resulting in large irreducible average bit error rates and outage rates unless a third explicit space diversity branch is added. The $M=2$, coded, non-interleaved system's irreducible average BER is found to be larger than the $M=2$, uncoded system's irreducible average BER. The 
reverse is however true in terms of outage rate. This results in a coding gain which is nil in terms of average BER but, strictly speaking, as high as $13 \mathrm{~dB}$ in terms of outage rate. However, a more stringent performance criterion (e.g. an outage rate of $10^{-3}$ ) makes the use of a third space diversity branch essential. Figure 5.10 then indicates that the $M=2$, coded system's average bit error rate at $\frac{E_{k}}{N_{11}}=30 \mathrm{~dB}$ only becomes smaller than the $M=2$, uncoded system's average bit error rate when the SIR becomes larger than $15.3 \mathrm{~dB}$. At that point, positive coding gains in terms of a $10^{-3}$ average BER should be achievable and the coded system's irreducible outage rate is sufficiently low to satisfy even a strict performance criterion of $10^{-3}$ outage rate.

As had been shown in Figure 2.1 of Chapter 2, the probability that one cochannel interferer makes up more than half of the total cochannel interference power while the contribution of other cochannel interferers remains small but non-negligible, can be quite significant. For example, with $15 \mathrm{~dB}<\operatorname{SIR}_{\mathrm{tot}}<10 \mathrm{~dB}$, one interferer contributes more than half the total interference power with probability 0.90 but does not exceed $90 \%$ of this total interference power with probability 0.55 . It is thus of interest to examine the influence of two unequal power interferers on the uncoded vs. coded, non-interleaved performance trade-off as is done in Figures 5.11 and 5.12 where the single interferer and two equal power interferer cases are also reproduced for purposes of comparison. It is seen that from the zero average BER coding gain observed with equal-power interferers, the average $B E R$ coding gain rises to $1 \mathrm{~dB}, 2.5 \mathrm{~dB}$ and 3.1 dB with respective power splits of $\left(\frac{5}{8}: \frac{3}{8}\right),\left(\frac{3}{4}: \frac{1}{4}\right)$ and $\left(\frac{7}{8}: \frac{1}{8}\right)$. These correspond to cases where the lower power interferer is $2.2 \mathrm{~dB}, 4.8 \mathrm{~dB}$ and $8.5 \mathrm{~dB}$ below the more powerful interferer. For these three power distributions and as is the case with two equal-power interferers, the coded system's irreducible average BER is larger than the uncoded system's irreducible average BER while the reverse is again true in terms of outage rate. Large outage rate coding gains are also achievable but not in terms of a more stringent performance criterion such as a $10^{-3}$ outage rate which does not seem attainable unless the lower power interferer makes up less than 1/8 of the total interference power i.e. unless this second interferer is at least $8.5 \mathrm{~dB}$ below the 
more powerful interferer ${ }^{74}$. It is then seen from Figures 5.13a and 5.14a that the coded system's average BER at $\frac{E_{0}}{N_{1}}=30 \mathrm{~dB}$ becomes lower than the uncoded system's average BER when the total SIR exceeds $15.3 \mathrm{~dB}, 14.6 \mathrm{~dB}, 13.5 \mathrm{~dB}, 11.8 \mathrm{~dB}$ and $8.5 \mathrm{~dB}$ for respective power splits of $\left(\frac{1}{2}: \frac{1}{2}\right),\left(\frac{5}{8}: \frac{3}{8}\right),\left(\frac{3}{4}: \frac{1}{4}\right),\left(\frac{7}{8}: \frac{1}{8}\right)$ and $(1: 0)$. In view of the results of Figures 5.11 and 5.12, positive average BER and outage rate coding gains $\mathbf{~}^{75}$ are therefore achievable above these SIR thresholds. These coding gains represent gains in $\frac{E_{b}}{N_{0}}$ achieved with a fixed SIR. On the other hand, Figures 5.13 and 5.14 can be used to determine coding gains in SIR for a fixed $\frac{E_{6}}{N_{11}}$ of $30 \mathrm{~dB}$. While average BER coding gains in SIR are not achievable as the coded system's average BER only becomes lower than the uncoded system's average BER when the latter is much smaller than $10^{-3}$, SIR coding gains in outage rate of $1.0 \mathrm{~dB}, 0.9 \mathrm{~dB}, 0.6 \mathrm{~dB}$ and $0.6 \mathrm{~dB}$ are achieved with power splits of $\left(\frac{1}{2}: \frac{1}{2}\right),\left(\frac{5}{8}: \frac{3}{8}\right),\left(\frac{3}{4}: \frac{1}{4}\right)$ and $\left(\frac{7}{8}: \frac{1}{8}\right)$ respectively. In terms of the more stringent performance criterion of $10^{-3}$ outage rate, these gains become $1.0 \mathrm{~dB}$, $1.0 \mathrm{~dB}, 1.2 \mathrm{~dB}$ and $1.2 \mathrm{~dB}$. Hence, in terms of the most relevant performance criterion for portable communications i.e. outage rate, coding gains with no interleaving are still observed in the presence of two interferers not only in terms of $\frac{F_{b}}{N_{11}}$ for a fixed SIR but also, with albeit quite smaller values, in terms of SIR for a fixed $\frac{E_{p}}{N_{11}}$.

At this point, all observations on coding gain have been based on a comparison of uncoded BPSK and rate $\frac{1}{2}$ coded QPSK thus maintaining the same bandwidth. If the coding gain comparisons are performed for a fixed modulation scheme, e.g. uncoded QPSK vs. rate $\frac{1}{2}$ coded QPSK, the influence of bandwidth expansion must be taken into account. In general, for a fixed total bandwidth allocation and system capacity requirement, the bandwidth expansion required for coding can only be obtained by decreasing the number of channels, which in turn decreases the cochannel reuse ratio $\frac{D}{R}$, i.e. the ratio of the distance $D$ between the centers of nearest neighboring cochannel cells to the cell radius $R$. This $\frac{D}{R}$ ratio, which is related to the number of cells $N_{c}$ (or channel sets out of the total bandwidth) per cluster by $\frac{D}{R}=\sqrt{3 N_{c}}$ for a hexagonal

74 As the irreducible outage rate in Figure $5.12 \mathrm{~b}$ is actually $1.6 \times 10^{-3}$ for the $\left(\frac{\pi}{8}: \frac{1}{4}\right)$ power split, it may be said that the second interferer must be at least $10 \mathrm{~dB}$ (i.e. a good "ballpark" ;igure) below the more powerful interferer to achieve a $10^{-3}$ outage rate.

75 Recall that these have been defined in terms of a $10^{-3}$ average BER and a $10^{-2}$ outage rate at a BER of $10^{-3}$. 
cellular geometry [18] should typically be made as small as possible to enhance system capacity but also as large as possible to increase the SIR on the radio links. In this sense, in order to maintain the same capacity for a given total bandwidth, the bandwidth expansion due to coding will result in a decrease of the SIR on the radio links. For a two-dimensional frequency reuse arrangement and a typical indoor path loss exponent of 4 (as discussed in Chapter 2), the variation in radio link SIR has been found to be [212]

$$
\Delta S I R=20 * \log _{10}\left(\frac{1}{R_{c}}\right)
$$

where $R_{c}$ is the code rate. As the rate $\frac{1}{2}$ code considered in this study yields a 6 dB reduction in SIR, Figure 5.15 compares uncoded QPSK with a 16 dB SIR to coded QPSK with a $10 \mathrm{~dB}$ SIR in the presence of a single interferer. For a fixed data (information) rate of $10 \mathrm{Mbits} / \mathrm{s}$, the symbol rate for the rate $\frac{1}{2}$ coded QPSK case remains at $10 \mathrm{Msymbols} / \mathrm{s}\left(\sigma_{\mathrm{d}}=0.45 \mathrm{~T}\right)$ but is now $5 \mathrm{Msymbols} / \mathrm{s}\left(\sigma_{\mathrm{d}}=0.225 \mathrm{~T}\right)$ for the uncoded QPSK case. While ideal interleaving enables the two-antenna coded system to outperform its uncoded counterpart by $7.3 \mathrm{~dB}$ in terms of average BER and by $13.5 \mathrm{~dB}$ in terms of outage rate and also outperform the three-antenna uncoded system by 2.7 $\mathrm{dB}$ in terms of average BER and by $7.4 \mathrm{~dB}$ in terms of outage rate, the non-interleaved coded system still yields positive coding gains with respect to the uncoded two-antenna system by respectively recuperating $45 \%(2.1 \mathrm{~dB})$ and $69 \%(4.2 \mathrm{~dB})$ of the average BER and outage rate gains obtained by adding a third space diversity branch. This compares with 54\% and 80\% observed in Figure 5.7 where uncoded BPSK had been compared to coded QPSK; in absolute terms, the coring gains are also approximately $1 \mathrm{~dB}$ smaller. Figure 5.16 then shows results at different SIR for $\frac{E_{b}}{N_{0}}=30 \mathrm{~dB}$; the coded curve is the same that appeared in Figure 5.8 but it has now been shifted by 6 $\mathrm{dB}$ to account for the bandwidth expansion. It is seen that coding with no interleaving can only be beneficial if the initial uncoded system's SIR is superior to $14.8 \mathrm{~dB}$. This is also approximately $6 \mathrm{~dB}$ higher than was found in Figure 5.8 for the uncoded BPSK to coded QPSK comparison. As irreducible average bit error rates and outage rates will also be observed with the uncoded QPSK to coded QPSK comparison in 
the presence of two interferers, the SIR threshold above which coding could be useful would probably rise to more than $20 \mathrm{~dB}$ from the $15 \mathrm{~dB}$ observed in Figure 5.10. Hence, the minimum SIR for which coding is a viable alternative to increasing the optimum baseband combiner's space diversity from two to three is approximately $6 \mathrm{~dB}$ higher for the uncoded QPSK-coded QPSK comparison than for the uncoded BPSKcoded QPSK comparison. It should also be noted, as shown in Figures 5.17 and 5.18, that a different symbol rate as well as cross-rail interference created by intersymbol interference and cochannel interference affect the relative performance of QPSK and BPSK with identical information throughput rates. With no cochannel interference as in Figure 5.17, QPSK degrades by no more than $1 \mathrm{~dB}$ with respect to BPSK in terms of both a $10^{-3}$ BER and a $10^{-2}$ outage rate; QPSK's 5 Msymbols/s signaling rate $\left(\sigma_{d}\right.$ $=0.225 \mathrm{~T}$ ) thus yields a smaller implicit diversity effect ${ }^{76}$ than BPSK's 10 Msymbols/s signaling rate $\left(\sigma_{d}=0.45 \mathrm{~T}\right)$. In the presence of one cochannel interferer as in Figure 5.18, cross-rail interference can severely degrade QPSK but, at low SIR, the degradation with respect to BPSK of twice the symbol rate is small. QPSK performance at a 16 dB SIR is seen to be only about one order of magnitude better than BPSK performance at a $10 \mathrm{~dB}$ SIR which is why the $\frac{E_{b}}{N_{0}}$ coding gains observed in Figure 5.15 (coded QPSK vs. uncoded QPSK) are only slightly smaller than those observed in Figure 5.7 (coded QPSK vs. uncoded BPSK). in addition, as BPSK with a $16 \mathrm{~dB}$ SIR yields the same BER as QPSK with a $22 \mathrm{~dB}$ SIR, $\frac{E_{b}}{N_{11}}$ coding gains at a $16 \mathrm{~dB}$ coded system SIR would then probably be quite similar for the coded QPSK-uncoded BPSK and coded QPSK-uncoded QPSK comparisons. The relative merit of using channel coding with respect to adding a third diversity thus depends on the minimum radio link SIR and on whether BPSK or QPSK is used.

\subsubsection{Effect of a lower data rate}

The effect of lowering the data and signaling rate to $1 \mathrm{Mbits} / \mathrm{s}$ on che coding vs. space diversity trade-off is shown on Figure 5.19. It is seen that the average BER coding gains are now $1.7 \mathrm{~dB}$ for $\mathrm{N}=1$ cochannel interferer and $1.5 \mathrm{~dB}$ for $\mathrm{N}=0$; in

76 Cross-rail interference from ISI also degrades QPSK with respect to BPSK, albeit slightly in this case as the harmful effects of increasing ISI are not yet observed at a symbol rate of 5 Msymbols/s. 
terms of outage rate, the coding gains are nnw $4.5 \mathrm{~dB}$ for $\mathrm{N}=1$ and $4.2 \mathrm{~dB}$ for $\mathrm{N}=0$. These reductions of $1 \mathrm{~dB}$ or more in coding gains with respect to their values at 10 Mbits/s (Figures 5.5 and 5.7) results from the equalizer's diminished ability to exploit the channel's implicit diversity effect at this lower signaling rate; i.e. the $\frac{T}{2}$ tap spacing now corresponds to $500 \mathrm{~ns}$ which is more than ten times the rms delay spread of $\mathbf{4 5}$ ns. However, the highly variable nature of the impulse responses generated with the cluster channel model is such that the benefits of exploiting implicit diversity are still felt somewhat at $1 \mathrm{Mbits} / \mathrm{s}$. This is confirmed in Figure 5.20 where the number of forward filter taps $L$ has been reduced to one; with only dual space diversity, the average BER coding gains are further reduced to $0.6 \mathrm{~dB}$ for $\mathrm{N}=1$ interferer and to 1.3 $\mathrm{dB}$ for $\mathrm{N}=0$ while in terms of outage rate, the coding gains are now $3.8 \mathrm{~dB}$ for $\mathrm{N}=1$ and $4.0 \mathrm{~dB}$ for $\mathrm{N}=0$. These results imply that a few channel realizations must have significant echoes at a delay of $500 \mathrm{~ns}$ or more. In fact, from equation 5.77 which describes the exponential probability density function of the cluster interarrival times $\left(T_{l}-T_{l-1}\right)$, the corresponding cumulative distribution function is simply found to be

$$
P_{r}\left(\left(T_{l}-T_{l-1}\right)<x\right)=1-\exp (-\Lambda x)
$$

The probability that the cluster interarrival time exceeds the tap spacing of 500ns is then $\exp \left(-\frac{500 \mathrm{~ns}}{200 \mathrm{~ns}}\right)=0.0821$ which implies that in 821 of 10000 impulse response realizations, the second cluster will be separated from the first cluster by more than $\frac{T}{2}$ thus enabling the equalizer to capture a second significant path in at least a few of these 821 realizations. Figure 5.21 then shows that the implicit diversity effect is even more important in producing significant coding gains when the SIR (with $N=1$ interferer) ranges from $10 \mathrm{~dB}$ to $15 \mathrm{~dB}$. For a SIR lower than $10 \mathrm{~dB}$, coded system performance degrades quickly irrespective of the implicit diversity effect whereas for a SIR greater than $15 \mathrm{~dB}$, the $\mathrm{L}=1$ case also seems to yield significant coding gains. It should however be noted that the $B E R$ improvements in the $L=3$ case are not as substantial as those observed in Figure 5.6 for a data rate of $10 \mathrm{Mbits} / \mathrm{s}$ where the implicit diversity effect is stronger. 


\subsubsection{Channel model}

The results of Chapters 3 and 4 were respectively obtained with a continuous channel model and a discrete channel model with nine taps spaced by half the rms delay spread. Both of these models were based on an exponential average delay profile. Although such a profile does not provide a very accurate representation of an actual indoor channel where the multipath echoes tend to arrive in clusters, its simplicity made it a satisfactory candidate for the analytical approach which involves many simplifying assumptions; the speed with which random realizations of the discrete model could be generated, also made it adequate for the bit-by-bit simulation which is inaccurate in a statistical sense. As the semi-analytical approach of this Chapter involves fewer assumptions while providing statistically significant results, it is far more conducive to establishing quantitative results as opposed to qualitative results or trends which was done in Chapters 3 and 4. This Chapter's results being mostly generated with the cluster model, it is thus of interest to compare results obtained with both models. As the $10 \mathrm{Msymbol} / \mathrm{s}$ signaling rate produced a mean ms delay spread of $0.45 \mathrm{~T}$ for the cluster model, the comparisons in Figures 5.22 and 5.23 are performed with the nine-tap model's rms delay spread also equal to $0.45 \mathrm{~T}$.

Figure 5.22 compares average BER and outage rate vs. $\frac{E_{b}}{N_{v}}$ curves in the presence of no interference as well as one and two interferers with a total SIR of $10 \mathrm{~dB}$. At an average BER of $10^{-3}$, the nine-tap model with exponential delay profile is optimistic with respect to the cluster modei by $2.0 \mathrm{~dB}$ (in $\frac{E_{b}}{N_{0}}$ ) for $\mathrm{N}=0$, by $2.2 \mathrm{~dB}$ for $\mathrm{N}=1$ and by $3.3 \mathrm{~dB}$ for $\mathrm{N}=2$ cochannel interferers. In terms of a $10^{-2}$ outage rate, the corresponding $\frac{E_{0}}{V_{i v}}$ improvements with the nine-tap model are $2.3 \mathrm{~dB}$ for $\mathrm{N}=0$ and $\mathrm{N}=1$, and $9.5 \mathrm{~dB}$ for $\mathrm{N}=2$. The latter value is large in view of the much lower (by more than a factor of 2) irreducible outage rate observed with the nine-tap model in the $\mathrm{N}=2$ case. Figure 5.23 then compares average BER vs. SIR curves at $\frac{E_{6}}{N_{11}}=30 \mathrm{~dB}$ with one and two interferers. The average BER obtained with the nine-tap model at an SIR of $10 \mathrm{~dB}$ is found to require, with the cluster model, SIR's of $10.5 \mathrm{~dB}$ and $11.8 \mathrm{~dB}$ for $\mathrm{N}=1$ and $\mathbf{N}=2$ interferers respectively.

Each of the 10000 realizations generated with the exponential delay profile nine- 
tap channel model yield, with a fixed tap spacing equal to half the ms delay spread of 0.45T in Figures 5.22 and 5.23, constant impulse response durations of 1.8T. This is slightly less than twice the equalizer span of $T$ obtained with the three $\frac{T}{2}-$ spaced equalizer taps. Recall from Chapter 3 that the effects of increasing symbol dispersion were found to be minor as long as the impulse response duration remained smaller than twice the equalizer span; as the response's duration exceeded this value, performance was found to degrade quickly ${ }^{77}$. On the other hand, the arrival of individual multipath echoes as well as the arrival of clusters of these echoes are random variables in the cluster channel inodel; as the mean arrival time for the second cluster is $200 \mathrm{~ns}=$ $2 \mathrm{~T}$, this implies that the duration of some of the 10000 impulse response realizations will often significantly exceed $2 \mathrm{~T}$. This phenomenon, coupled with the variable path spacing which can affect the equalizer's ability to resolve the multiple paths for a given realization, thus tends to make results obtained, with the simplified nine-tap channel model optimistic with respect to those generated with the more realistic cluster model. However, it should be noted that while this conclusion holds at a data rate of $10 \mathrm{Mbits} / \mathrm{s}$, the cluster model will most probably yield improved results in terms of coding gain, with respect to the nine-tap model, as the data rate is lowered from $10 \mathrm{Mbits} / \mathrm{s}$. In such cases, the total dispersion eventually becomes smaller than the equalizer span but the nine-tap model's shorter impulse responses means that the implicit diversity effect will be lost at a higher data rate than with the cluster model.

\subsubsection{Interference model}

The purely analytical approach of Chapter 3 involved an exponential upper bound on the realizable performance of coherent BPSK and QPSK and a non-fading gaussian characterization of interference ${ }^{78}$. As this Chapter's semi-analytical approach eliminates these assumptions, it is worthwhile to assess their influence on performance predictions. Figure 5.24a thus compares the average BER vs. $\frac{E_{b}}{N_{11}}$ curves for both techniques in the absence of any cochannel interference (i.e. $\mathrm{N}=0$ ). It is seen that the andytical results are

77 Refer to Chapter 3, e.g. Figure 3.9 on page 90 where with rms delay spre $s$ of $0.25 \mathrm{~T}$ and $0.50 \mathrm{~T}$, the impulse response duration is respectively equal to, and twice the equalizer span of $T$.

7 Recall that this applies to intersymbol interference as well as cochannel interference. 
pessimistic with respect to the semi-analytical results. The difference, which is found to be $2.7 \mathrm{~dB}$ at a BER of $10^{-6}$, is a consequence of both the exponential bound on coherent BPSK employed in Chapter 3 as well as the choice of the gaussian interfering symbols' mean-square value $\gamma^{2}$, which was set to 0.5 . The influence of the exponential performance bound can be assessed by referring to Appendix B where BPSK and DPSK theoretical BER curves are compared in flat Rayleigh fading with no cochannel interference. Figure B.1a shows that, with $M=2$ antennas, BPSK outperforms DPSK by $2.8 \mathrm{~dB}$ at a BER of $10^{-6}$; the choice of $\gamma^{2}=0.5$ as the mean-square value of the intersymbol and cochannel interfering symbols thus appears to be reasonable. Further insight into the reliability of the analytical procedure can be gained by comparing the distributions of the signal-to-inteference-plus-noise ratio (SINR) at the decision device input $^{79}$ obtained from both techniques. The SINR distribution at high $\frac{E_{b}}{N_{t}}$, i.e. 30 $\mathrm{dB}$, is of particular interest as it represents the case where intersymbol and cochannel interference are dominant with respect to AWGN. The probahility de : ity function (pdf) of the SINR in the analytical procedure is determined by inverting the partial fraction expansion of its Laplace transform in eq. 3.44 (page 65); for this Chapter's semi-analytical procedure, it is simply determined from the SINR obtained via equation 3.37 (page 62) at each of the 10000 sets of portable to base station antenna channel realizations. It is thus seen in Figure 5.24b that, although the SINR at $\frac{E_{0}}{N_{1}}=30 \mathrm{~dB}$ ranges from 12 or $13 \mathrm{~dB}$ to 38 or $39 \mathrm{~dB}$ in both cases, the mean SINR is lower in the case of the analytical procedure thus resulting in worse performance.

However, Figures 5.25 and 5.26 clearly show the limitations of the analytical approach in the presence of cochannel interference. At low $\frac{E_{6}}{N_{11}}$ where AWGN dominates, the relative positions of the BER curves are similar to the $\mathrm{N}=0$ case. On the other hand, at high $\frac{E_{b}}{N_{t}}$, the analytical curve becorses optimistic and overly so in the $\mathrm{N}=2$ case as it fails to predict the irreducible BER. This results from the deterministic nature of the interference in the analysis, i.e. with a non-fading characterization of interference, the analysis only accounts for short-term variations in the desired signal's power level and not in the cochantel interferers' power level. In fact, the SINR pdf plots in Figures

79 This corresponds to point $z$ in Figuie " page 52. 
3 of/de

$$
\text { 政施 }
$$


$5.25 \mathrm{~b}$ and $5.26 \mathrm{~b}$ show that the semi-analytical method of moments technique, which accounts for the fading of both the desired signal and the interference, produces some realizations with SINR's much lower than predicted by analysis. These low SINR realizations tend to dominate the average BER and lead to worse performance than predicted by the analytical procedure.

Hence, it appears that the non-fading representation of interference is the dominant drawtack of the analytical approach. The gaussian characterization has a smaller influe $1 c e$ as the mean-square value $\gamma^{2}$ of the interferii.g symbols can be adjusted to yield good " $i a l l p a r k$ " estimates of performance as long as the number of cochannel interferers is small and the total SIR sufficiently high; i.e. with $M=2$ antennas, $N=1$ interferer and a SIR of $10 \mathrm{~dB}$, the analysis still yields a good estimate of performance over the BER range of interest $\left(\mathrm{i}^{-1}\right.$ to $\left.10^{-6}\right)$ while with $\mathrm{N}=2$ equal-power interferers, the results of Figure 5.8a indicate that the analysis could probably also yield a reasonably good performance estimate, over a BER range of $10^{-1}$ to $10^{-6}$, for a total SIR greater than $15 \mathrm{dE}$ as the average BER at $\frac{E_{b}}{N_{11}}=30 \mathrm{~dB}$ is then smaller than $10^{-6}$.

At this point, the gaussian assumption of interference warrants some clarification. As stated in Chapter 3, this thesis assuries fixed large-scale mean signal and interference levels. It does not account for the large-scale shadowing effects which are assumed to be tandled by macrnscopic diversity. As the objective of this work consists of evaluating microsscopic diversity techniques on the radio links, only small-scale fading variations are considered. The non-gaussian nature of cochannel interference is certainly true in a large-scale sense as even the sum of a moderate but finite number (e.g. up to 12) of log-normally distributed signal envelopes has been found to be well approximated by another log-normal variate [115, 116]. On the other hand, in a small-scale sense i.e. with fixed large-scale means, each interferer is simply a complex gaussian variate with a Rayleigh or Rician envelope and a uniform phase. This is certainly true in the long-term, i.e. as an average over the fading channel statistics. However the short-term characteristics of interference, i.e. over a period smaller than the averz.ge fade duration where the channel can be considered as static, were assumed gaussian in the analysis of Chapter 3 by approximating the short-term interference covariance matrix components 
of equation 3.30 by their long-term means over the channel statistics as in equation 3.38. This small-scale, short-term gaussian assumption of interference is obviously invalid in the presence of a single (or most probably of two) dominant interferer(s). In more ad-hoc terms, one may simply consider expressing the probability of error averaged over the long-term interference statistics $P(I)$ as

$$
\overline{P(\text { error })}=P(\text { error } \mid I) P(I)
$$

Although the interference consists of complex gaussian variates in the long-term, the short-term probability of error $P($ error $\mid I)$ will be erroneous if the interference is presumed to be short-term gaussian distributed which is not the case unless there are many significant interferers.

\subsubsection{Sampling phase}

All the results presented up to this point have been based on a fixed and nearly optimal receiver sampling phase $t_{0}$. As shown in Chapter 3 , with only $L=3$ forward filter taps and a rms delay spread on the order of $0.25 \mathrm{~T}$, the results' sensitivity to sampling phase can be quite significant particularly for large pulse shape excess bandwidths. It is thus worthwhile to determine if significant performance improvements could be achieved by continuously optimizing this receiver sampling phase. Figures 5.27 to 5.26 thus show average BER and outage rate results for the best and worst fixed sampling phases (out of 8 across the symbol interval $T$ ) as well as for the case of the continuously optimized sampling phase where, at each of the 10000 channel realizations, the selected sampling phase yielded the lowest BER (again for 8 different values of $t_{0}$ over the symbol interval) for that particular static channel. As the latter procedure substantially increases the computational burden, the results of Figures 5.27 to 5.30 are restricted to the single antenna $(M=1)$, no cochannel interference $(N=0)$ case with three $(L=3)$ forward filter taps and the simple exponential delay profile, nine-tap channel model. The results deal with rms delay spreads of $0.25 \mathrm{~T}$ and $0.50 \mathrm{~T}$, i.e. the range of interest in this work.

Figure 5.27 shows that, with the wide bandwidth time-rectangular pulse and $\sigma_{d}=0.25 \mathrm{~T}$, the continuously optimized sampling phase scheme yields a gain of 2.1 
$\mathrm{dB}$ at an average BER of $10^{-3}$ over the best fixed sampling phase case which in turn outperforms the worst fixed sampling phase case by $3.6 \mathrm{~dB}$. In terms of a $10^{-2}$ outage rate, these gains are respectively $2.3 \mathrm{~dB}$ and $2.9 \mathrm{~dB}$. When the rms delay spread is increased to 0.50T as in Figure 5.28, the continuously optimized sampling phase scheme now produces an average BER gain of $2.2 \mathrm{~dB}$ and a outage rate gain of $2.4 \mathrm{~dB}$ with respect to the best fixed sampling phase case; the latter yields an average BER gain of $4.1 \mathrm{~dB}$ and an outage rate gain of $3.1 \mathrm{~dB}$ over the worst fixed sampling phase case.

When a $50 \%$ rolloff SRC pulse shape is employed as in Figures 5.29 and 5.30, continuously optimizing the sampling phase with $\sigma_{d}=0.25 \mathrm{~T}$ now only yields an average BER gain of $0.7 \mathrm{~dB}$ and an outage rate gain of $0.5 \mathrm{~dB}$ with respect to the best fixed sampling phase case which in turn outperforms the worst fixed sampling phase case by $2.8 \mathrm{~dB}$ in terms of average BER and by $3.0 \mathrm{~dB}$ in terms of outage rate. With $\sigma_{d}=0.50 \mathrm{~T}$, the continuously optimized sampling phase scheme also produces an average BER gain of $0.7 \mathrm{~dB}$ and an outage rate gain of $0.5 \mathrm{~dB}$ over the best fixed sampling phase case which yields an average BER gain of $3.1 \mathrm{~dB}$ and an outage rate gain of $3.4 \mathrm{~dB}$ over the worst fixed sampling phase case.

Hence, while the continuously optimized sampling phase scheme yields significant gains (i.e. greater than $2 \mathrm{~dB}$ ) with respect to the best fixed sampling phase with the time-rectangular pulse, these gains become smaller than $1 \mathrm{~dB}$ with the $50 \%$ SRC pulse. However, as fixed sampling phase sensitivity remains non-negligible (i.e. in the 3 $\mathrm{dB}$ range) even with the $50 \%$ SRC pulse ${ }^{80}$, a continuously optimized sampling phase can thus prevent performance losses of $3 \mathrm{~dB}$ to $4 \mathrm{~dB}^{81}$. These losses could actually be higher with the more realistic cluster channel model where the greater variability of the impulse response realizations could lead to larger sampling phase sensitivity. Moreover, the latter tends to increase, as seen in Chapters 3 and 4 , in the presence of cochannel interference and with coding, but also decreases with a larger number of antennas $M$ and a larger number of taps per forward filter $L$. In practice, the sampling

80 Recall from Chapter 2 that sampling phase sensitivity typically increases with excess bandwidth.

81 Note that this holds for ms delay spreads $\left(\sigma_{d}\right)$ of $0.25 T$ and $0.50 T$. In fact, recall from Chapter 3 that, with $L=3$ taps, increasing $\sigma_{\mathrm{d}}$ from $0.25 \mathrm{~T}$ to $0.50 \mathrm{~T}$ only produced a small performance degradation which however became larger as $\sigma_{d}$ was increased beyond $0.50 \mathrm{~T}$. 
phase could be optimized by comparing the mean-square errors resulting from a few (e.g. eight) different sampling phases. Although the latter could be feasible in view of the very slow channel variations, it may be simpler to simply estimate the impulse response and set the sampling phase to the peak of the response. However, with two or more antennas (i.e. $M \geq 2$ ), this second approach appears problematic as independent impulse response realizations are obtained at each space diversity branch and setting the sampling phase to the peak of either response may affect performance differently. 


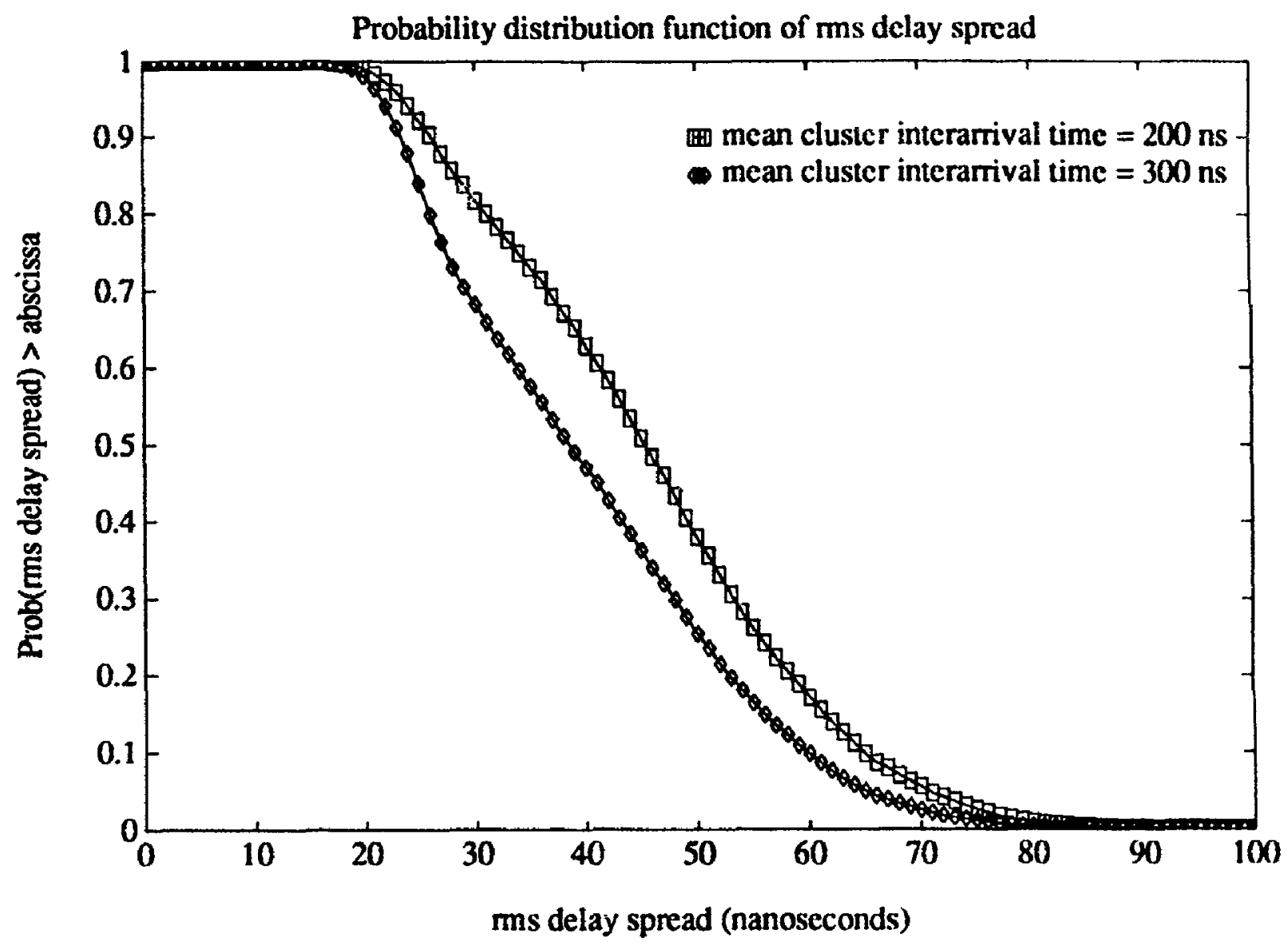

Figure 5.1 Probability distribution function of rms delay spread over 10000 impulse response realizations for two different mean cluster interarrival times. $(1 / \lambda)=5 \mathrm{~ns}, \Gamma=60 \mathrm{~ns}, \gamma=20 \mathrm{~ns}$. 
Average bit error rate vs. Eb/NO(dB)

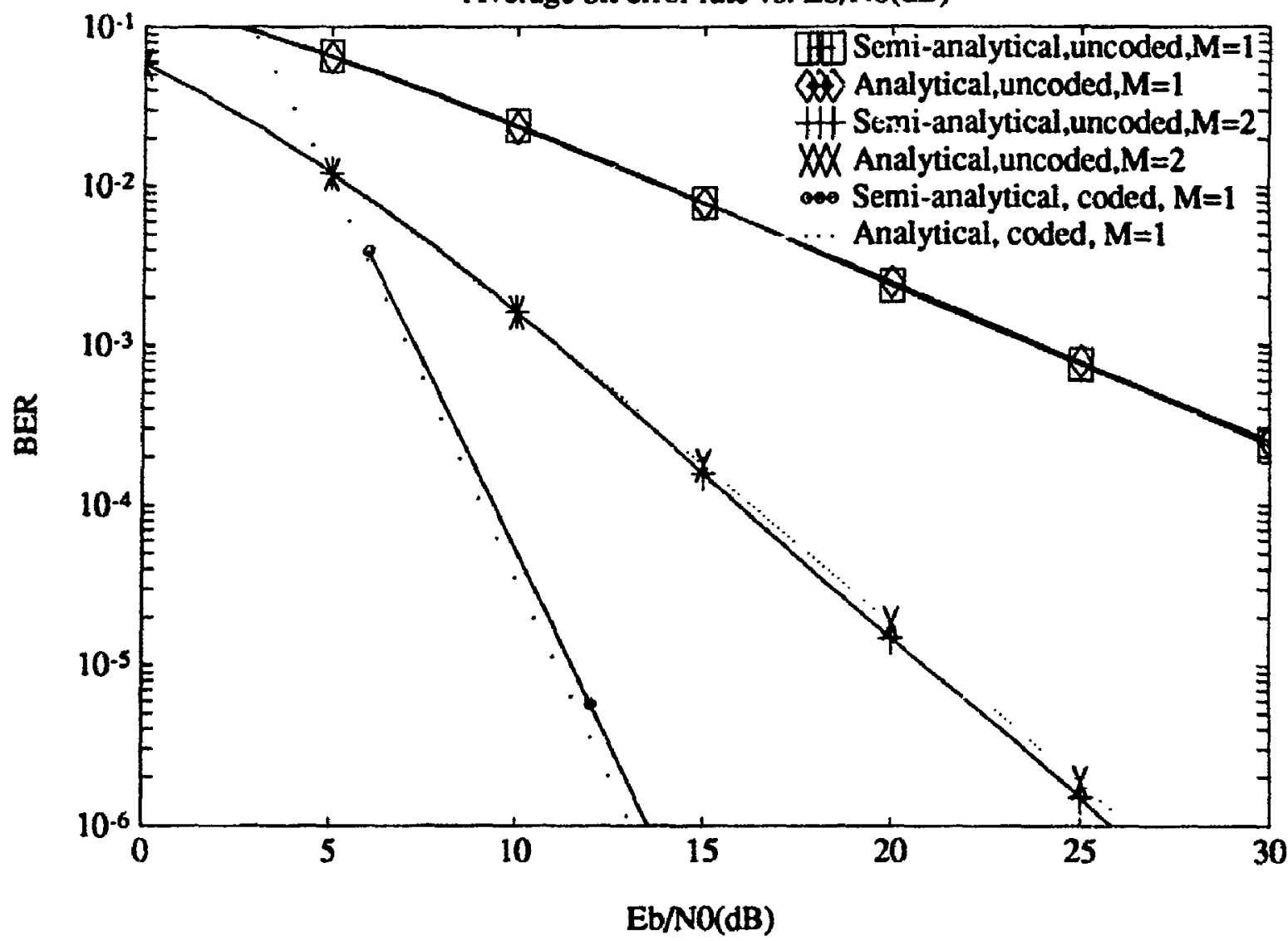

Figure 5.2 Test cases with BPSK, N=0, L=1, time-rectangular pulse shape and flat Rayleigh fading ( $\sigma_{d}=10^{-6} \mathrm{~T}$ with 9-tap channel model). $\mathbf{M}=\#$ of receiver antennas. Coded curves with ideal interleaving. 

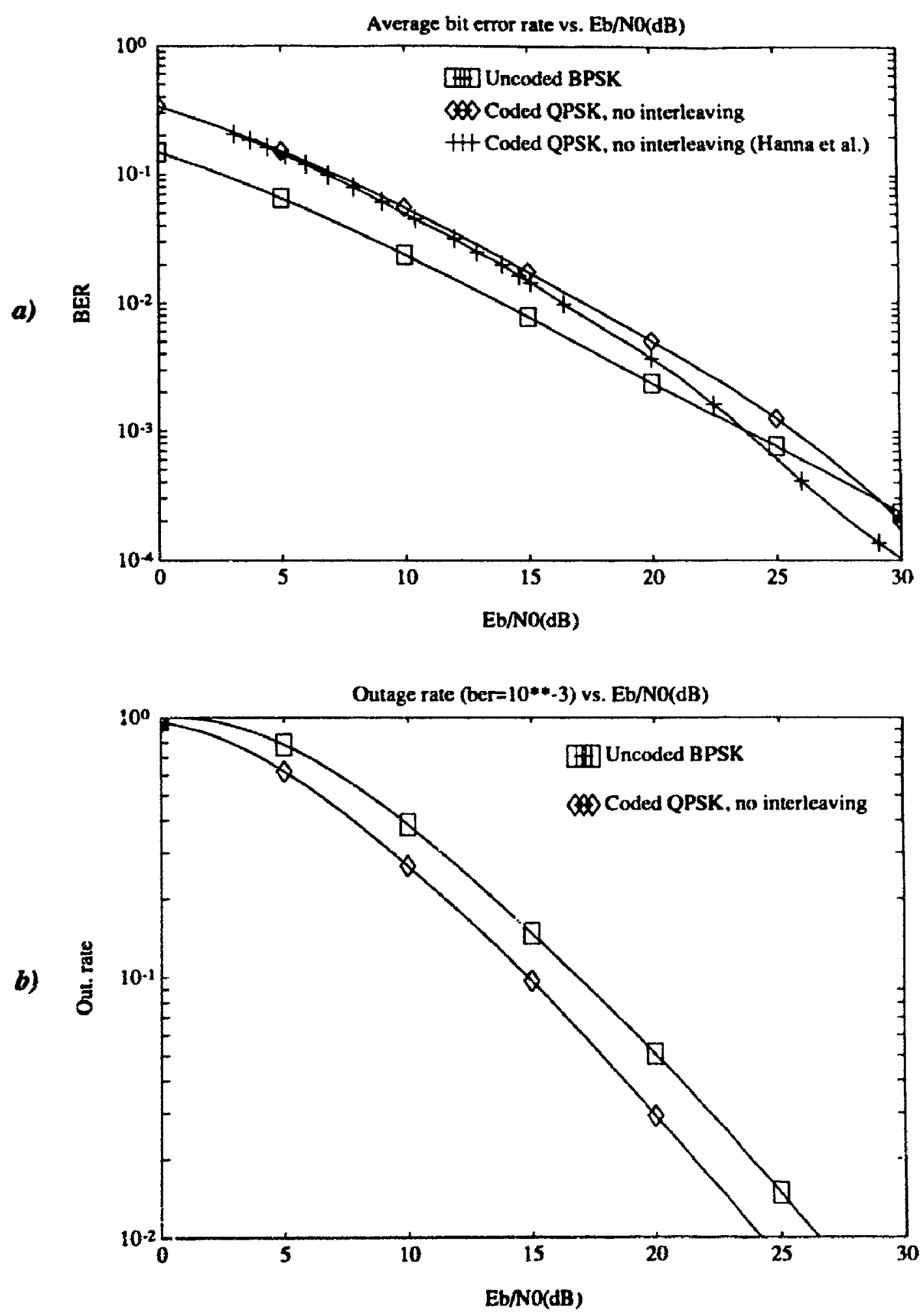

Figure 5.3 Test cases with $M=1, N=0, L=1$, time-rectangular pulse shape and flat Rayleigh fading $\left(\sigma_{d}=10^{-6} \mathrm{~T}\right.$ with 9-tap channel model). Hanna et al. results from [150] a) Average BER b) Outage rate 

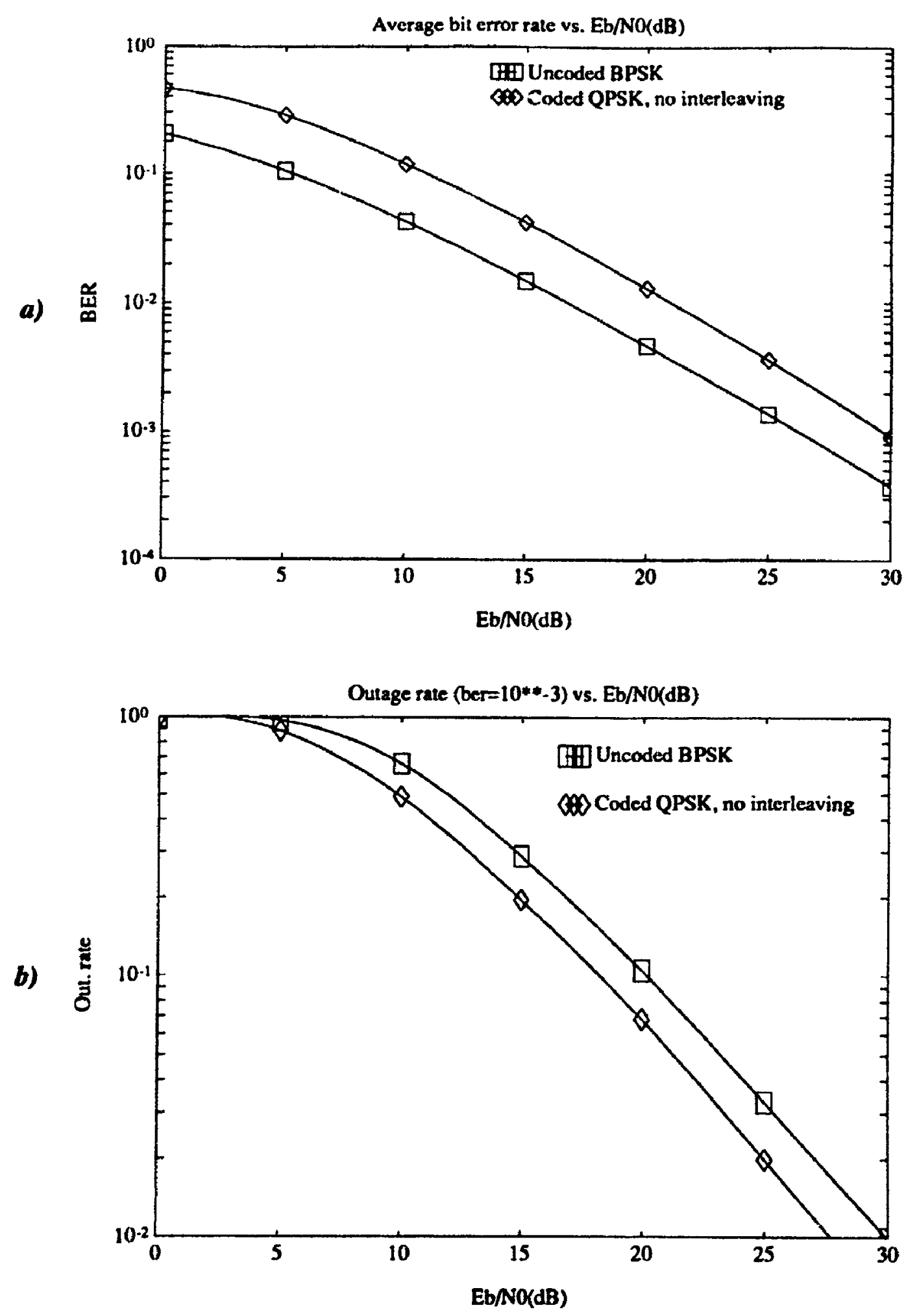

Figure 5.4 Test cases with $M=1, N=0, L=1, S R C$ (50\%) pulse shape and flat Rayleigh fading $\left(\sigma_{d}=10^{-6} \mathrm{~T}\right.$ with 9-tap channel model). a) Average BER b) Outage rate 


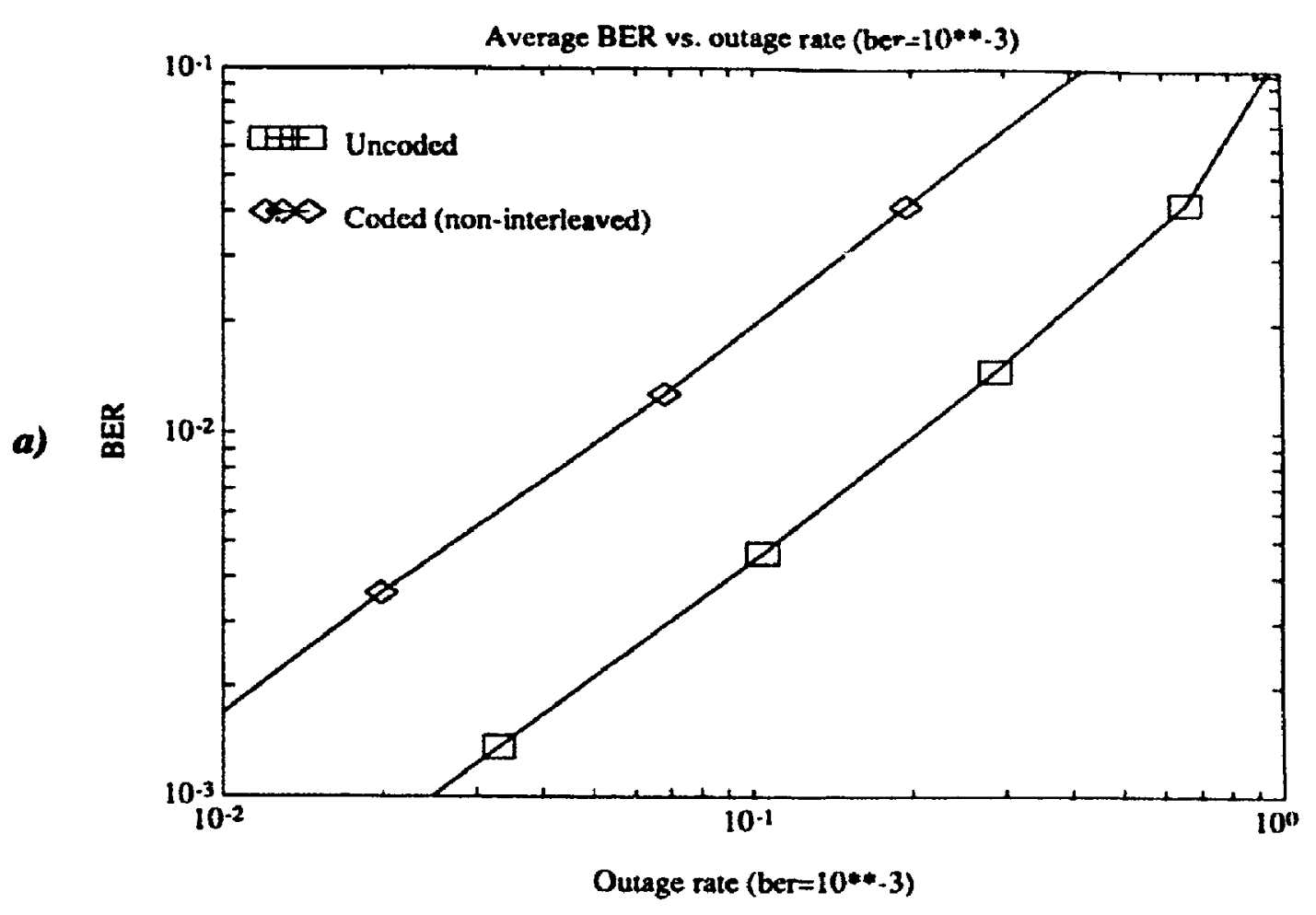

174

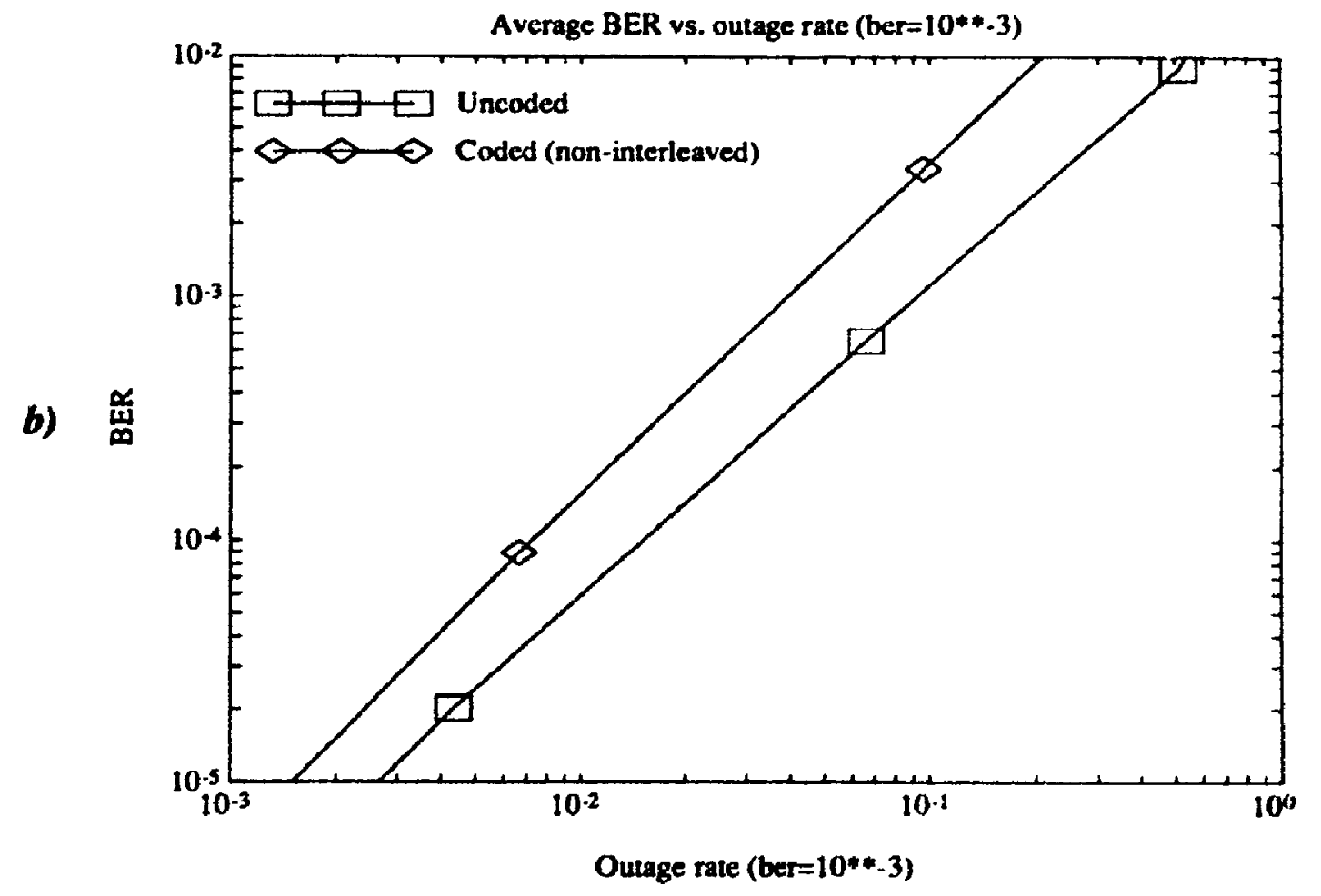

Figure 5.5 Average BER vs. outage rate at same value of Eb/No. $N=0, \operatorname{SRC}(50 \%)$ pulse shape. a) $M=1, L=1$, flat fading b) $M=2, L=3$, cluster channel model (10 Mbits/s data rate). 

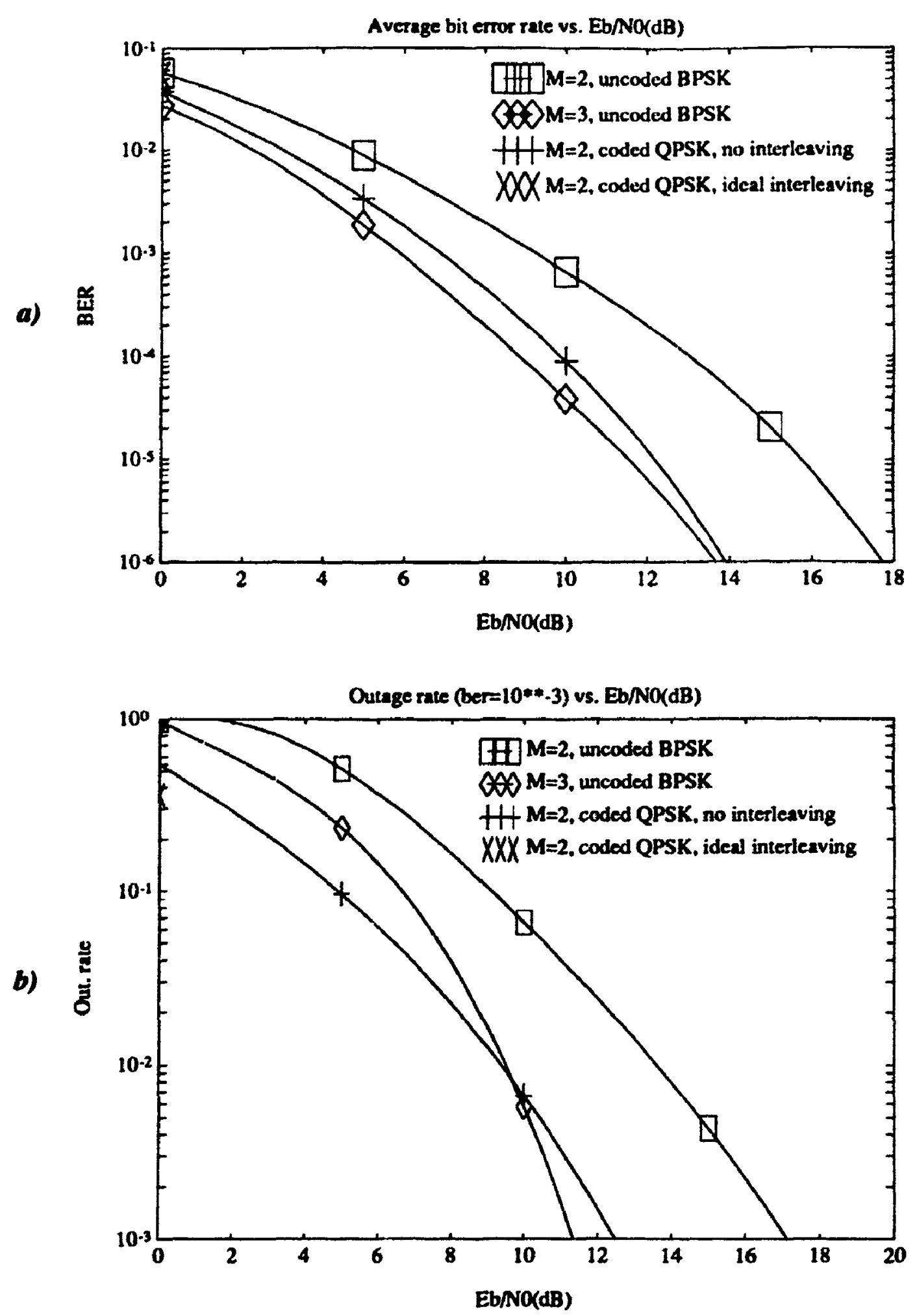

Figure 5.6 Results for no cochannel interierence $(N=0)$. $L=3$, cluster channel model (10 Mbits/s data rate), SRC $(50 \%)$ pulse shape. a) Average BER b) Outage rate 

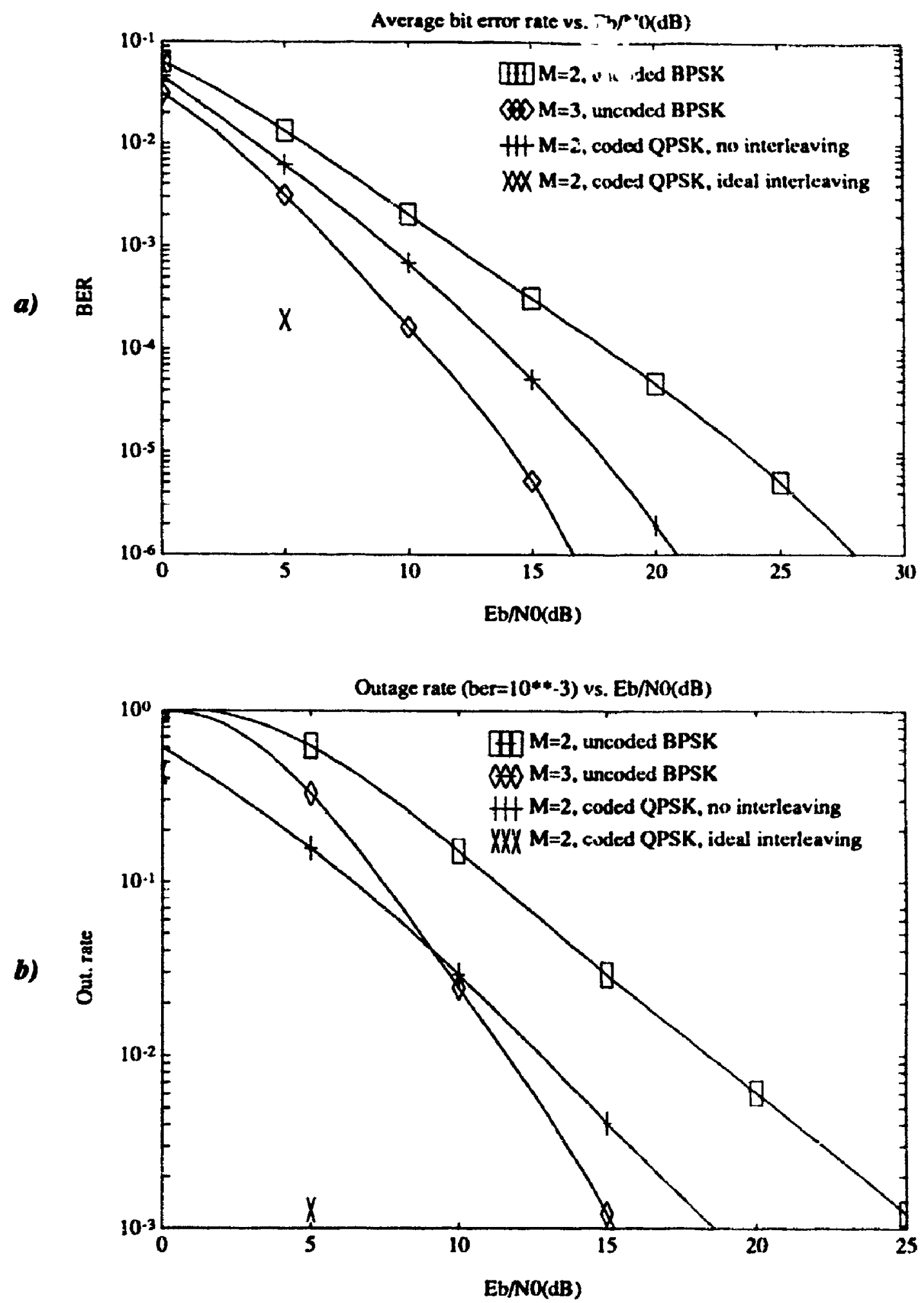

Figure 5.7 Results for $\mathbf{N}=1$ interferer.

SIR=10 dB, L=3, cluster channel model (10 Mbits/s data rate), SRC (50\%) pulse shape. a) Average BER b) Outage rate 


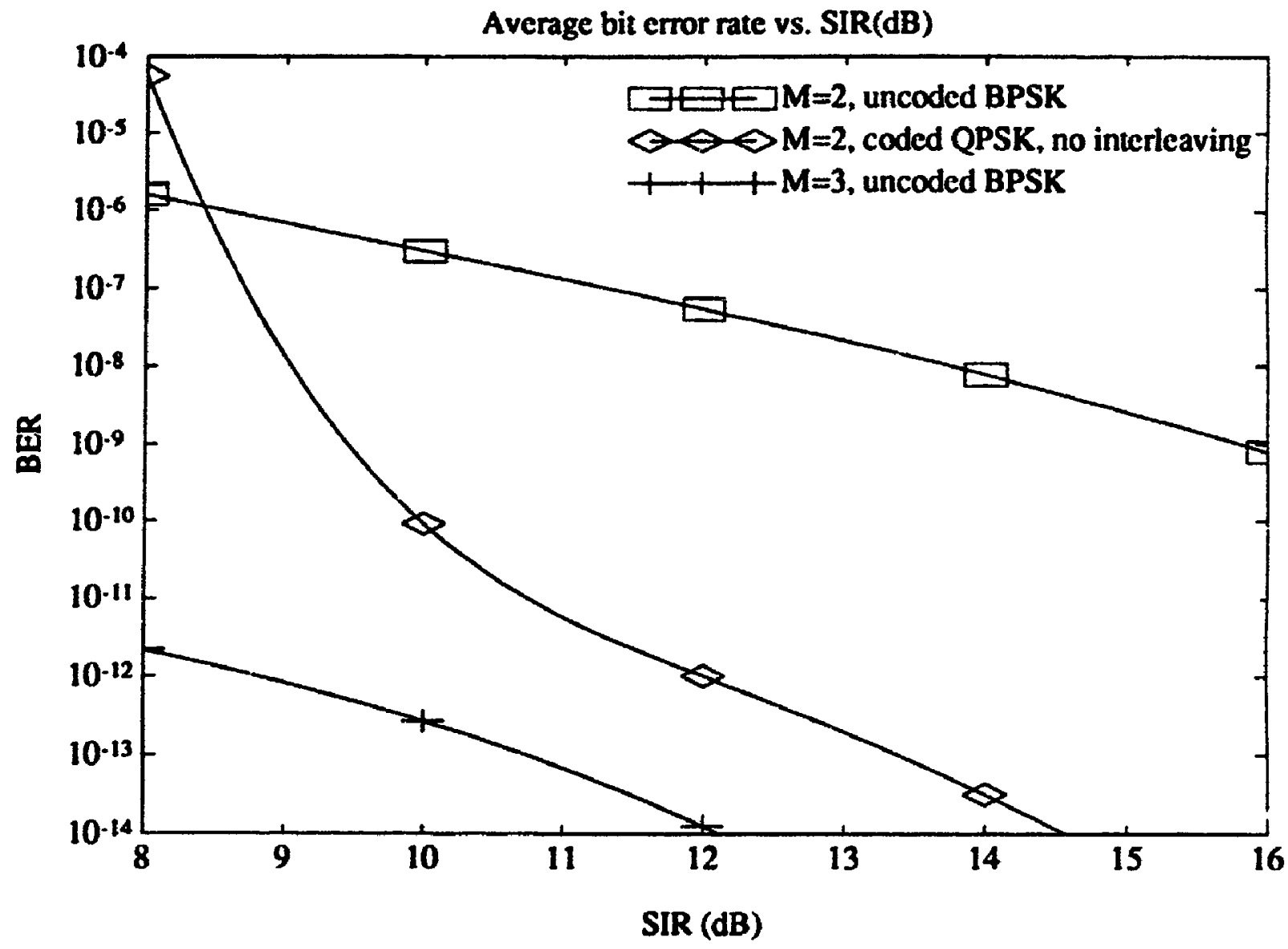

Figure 5.8 Results for $\mathrm{N}=1$ interferer.

$E b / N 0=30 \mathrm{~dB}, L=3$, cluster channel model (10

Mbits/s data rate), SRC (50\%) pulse shape. 

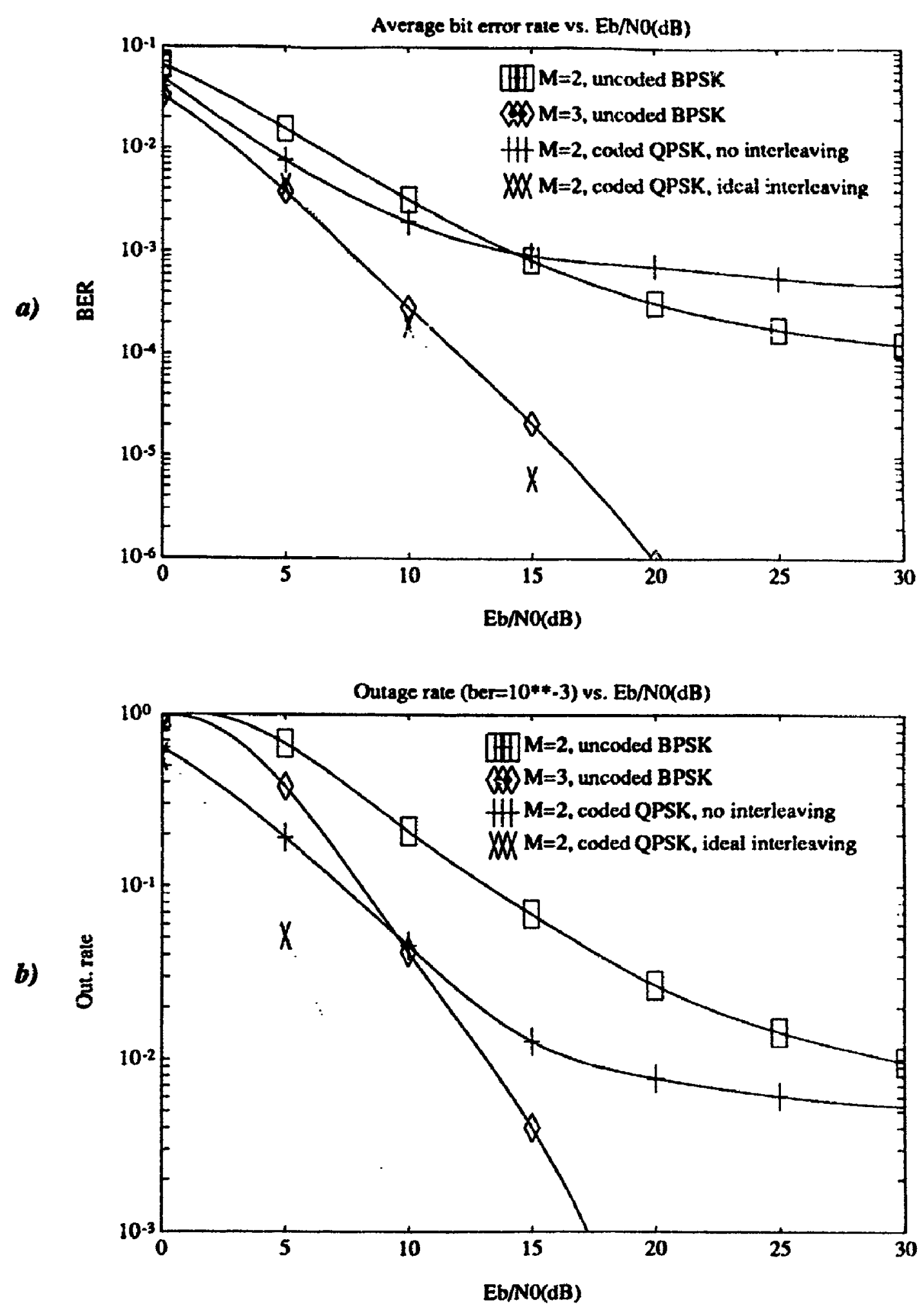

Figure 5.9 Results for $\mathrm{N}=2$ equal-power interferers.

$S_{\text {Itot }}=10 \mathrm{~dB}, \mathrm{~L}=3$, cluster channel model (10 Mbits/s data rate), SRC (50\%) pulse shape. a) Average BER b) Outage rate 

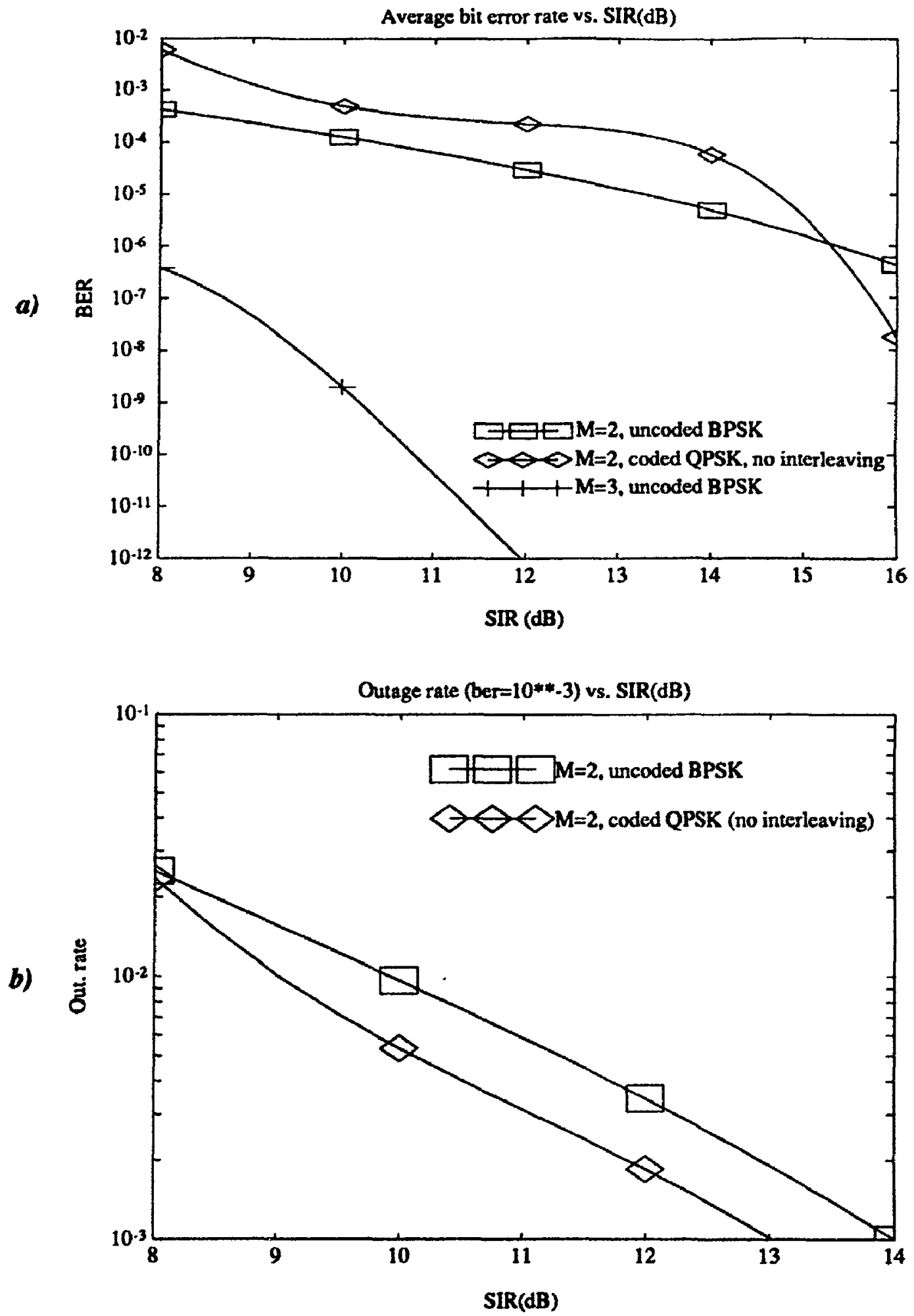

Figure 5.10 Results for $\mathrm{N}=2$ equal-power interferers. $\mathrm{Eb} / \mathrm{NO}=\mathbf{3 0}$ dB, L=3, cluster channel model (10 Mbits/s data rate), SRC (50\%) pulse shape. a) Average BER b) Outage rate 

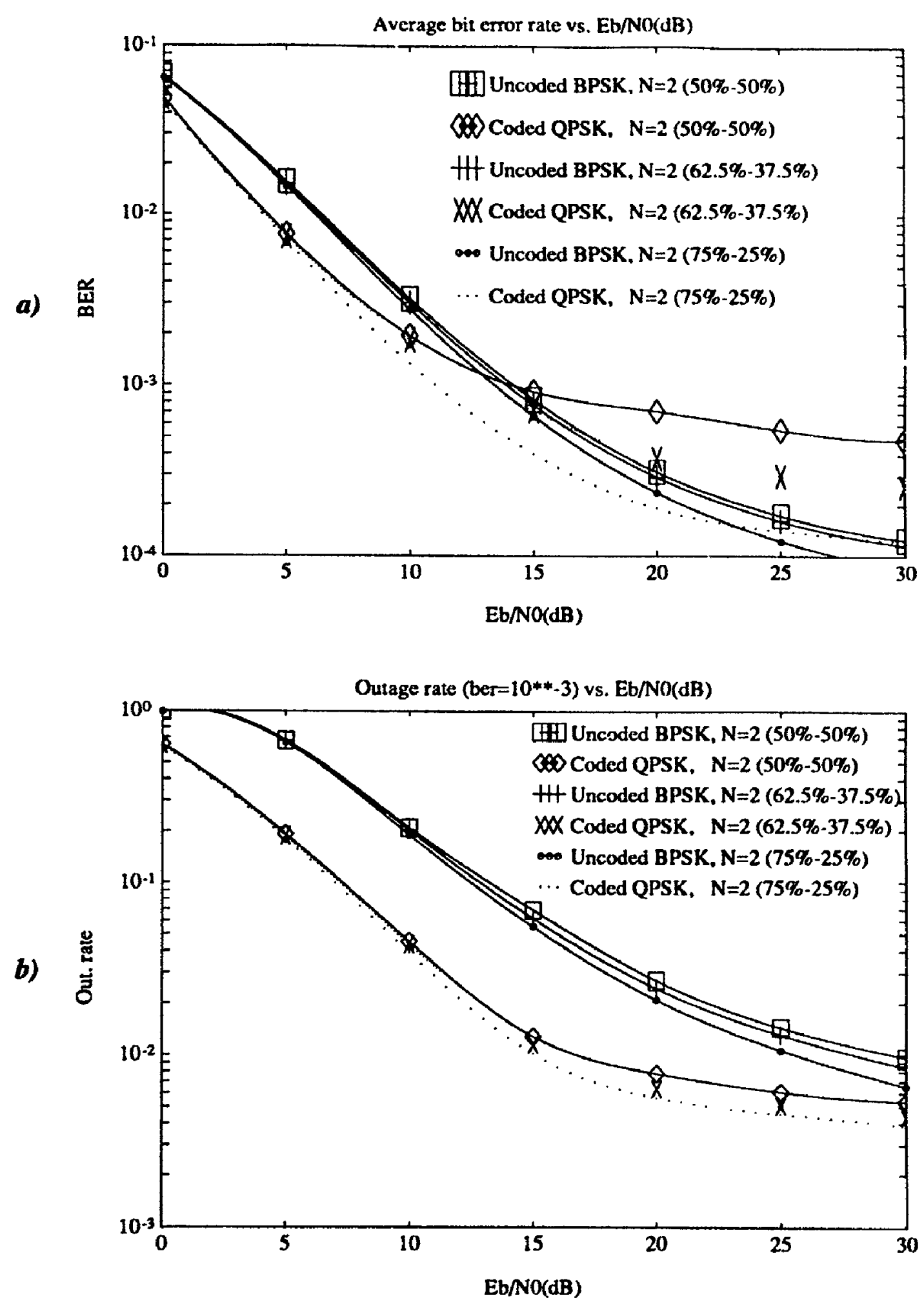

Figure 5.11 Results for $\mathbf{N}=2$ interferers with different power distributions. $S_{\text {tot }}=10 \mathrm{~dB}, M=2, L=3$, cluster channel model (10 Mbits/s data rate), $\mathrm{SRC}$ (50\%) pulse shape. Coded results with no interleaving. a) Average BER b) Outage rate 

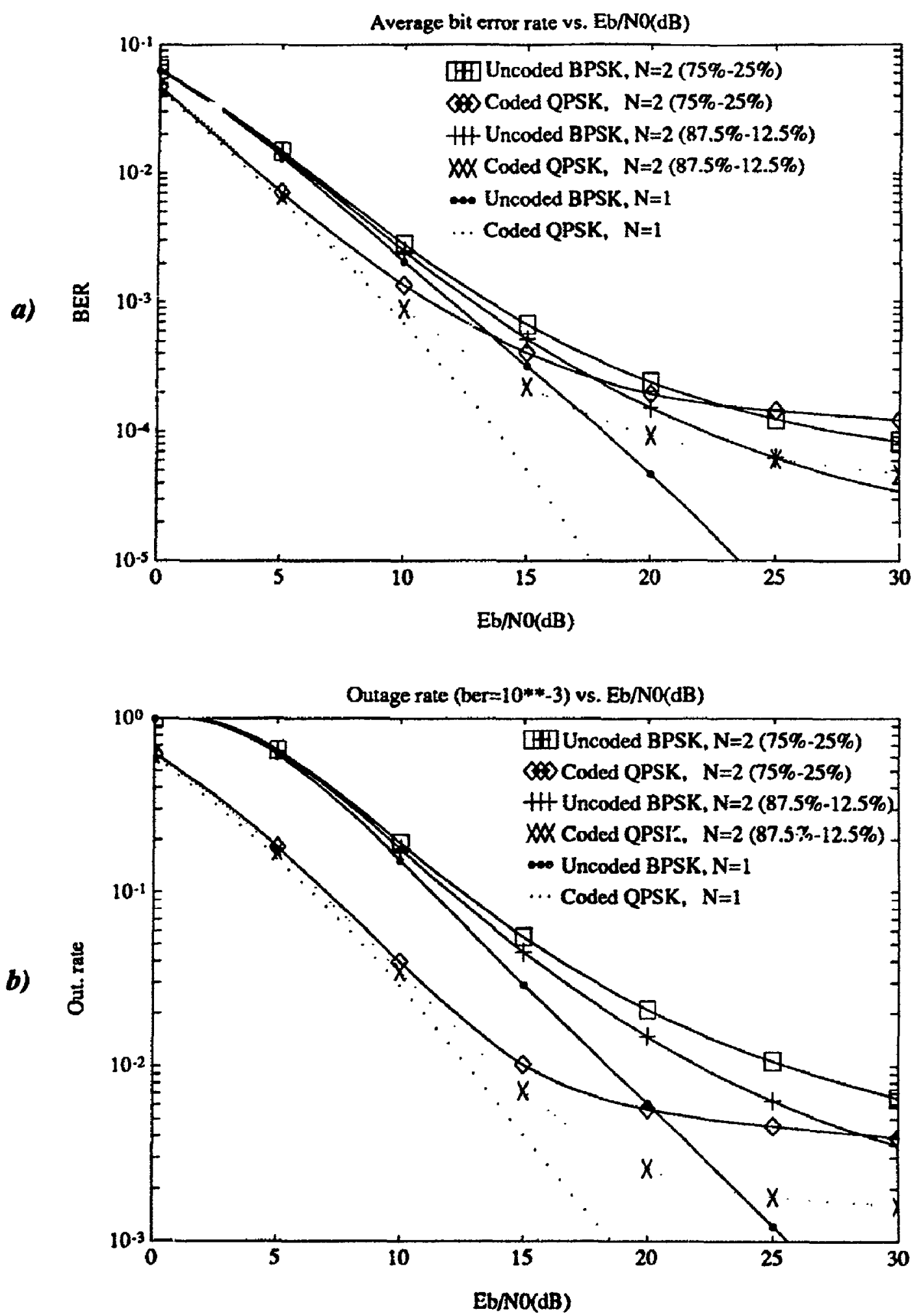

Figure 5.12 Results for $\mathrm{N}=2$ interferers with different power distributions. $S_{1 R_{10 t}}=10 \mathrm{~dB}, M=2, L=3$, cluster channel model (10 Mbits/s data rate), $S R C$ (50\%) pulse shape. Coded results with no interleaving. a) Average BER b) Outage rate 


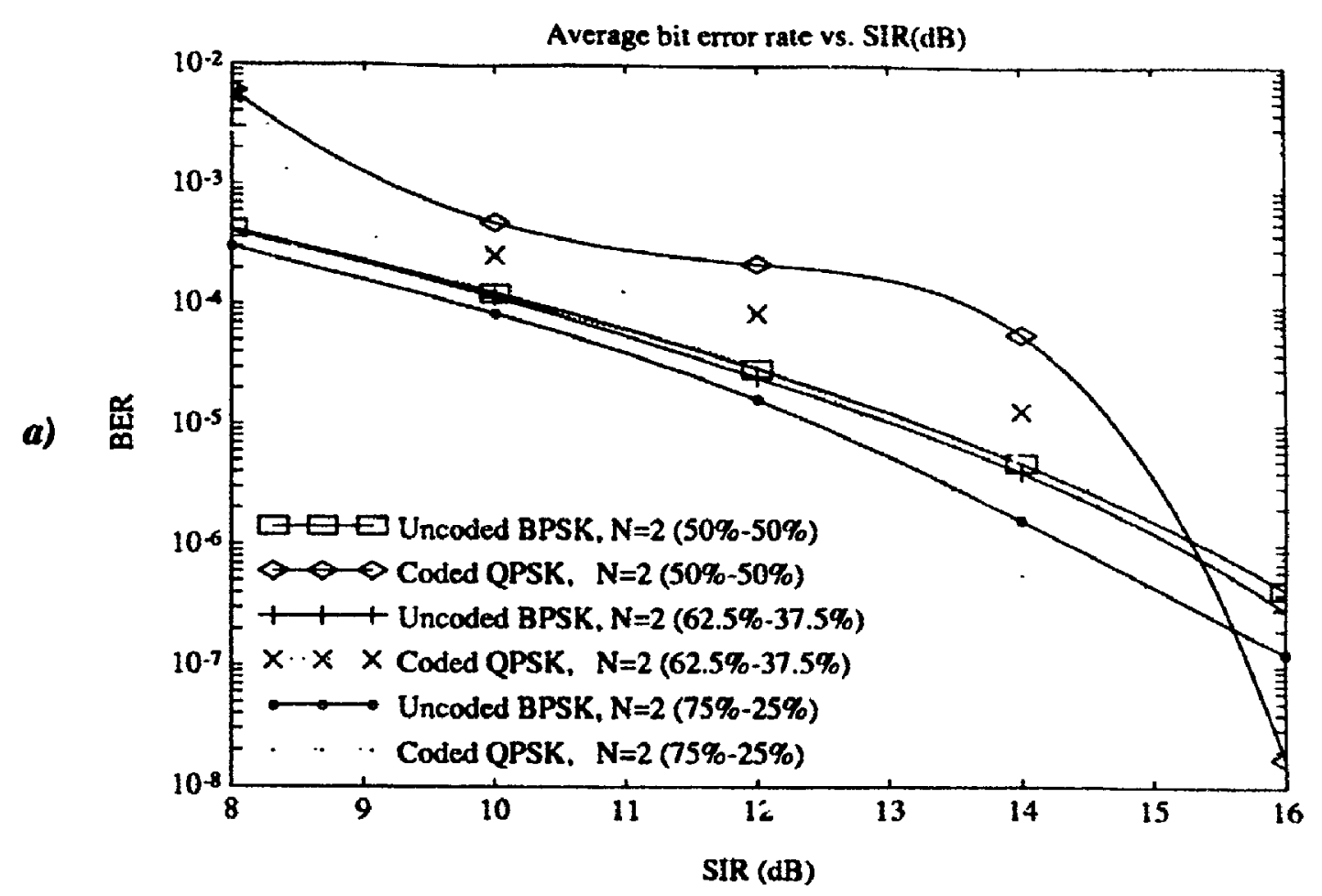

182

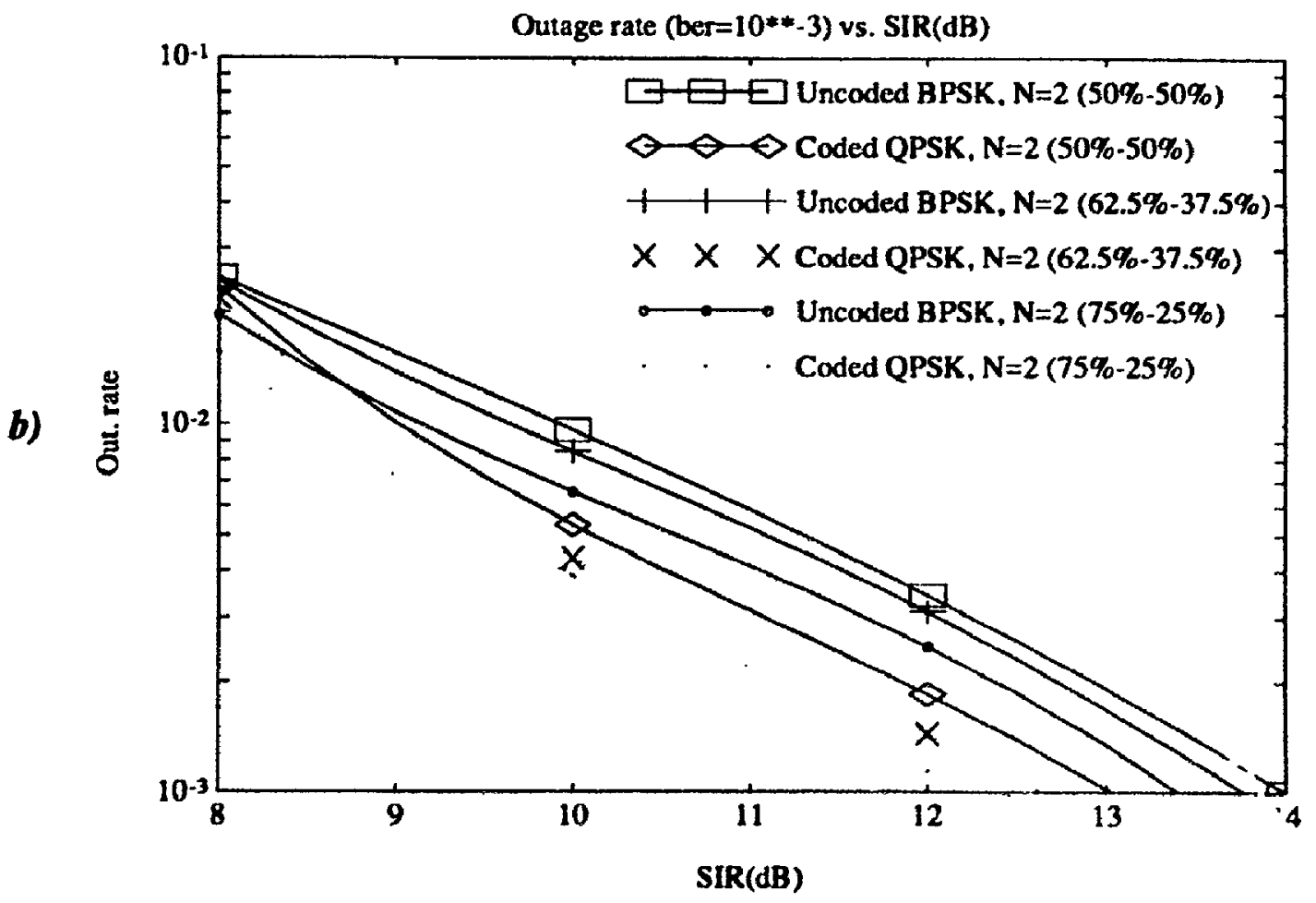

Figure 5.13 Results for $\mathrm{N}=2$ interferers with different power distributions. $\mathrm{Eb} / \mathrm{N} 0=30 \mathrm{~dB}, \mathrm{M}=2, \mathrm{~L}=3$, cluster channel model (10 Mbits/s data rate), $\mathrm{SRC}(50 \%)$ pulse shape. Coded results with no interleaving. a) Average BER b) Outage rate 

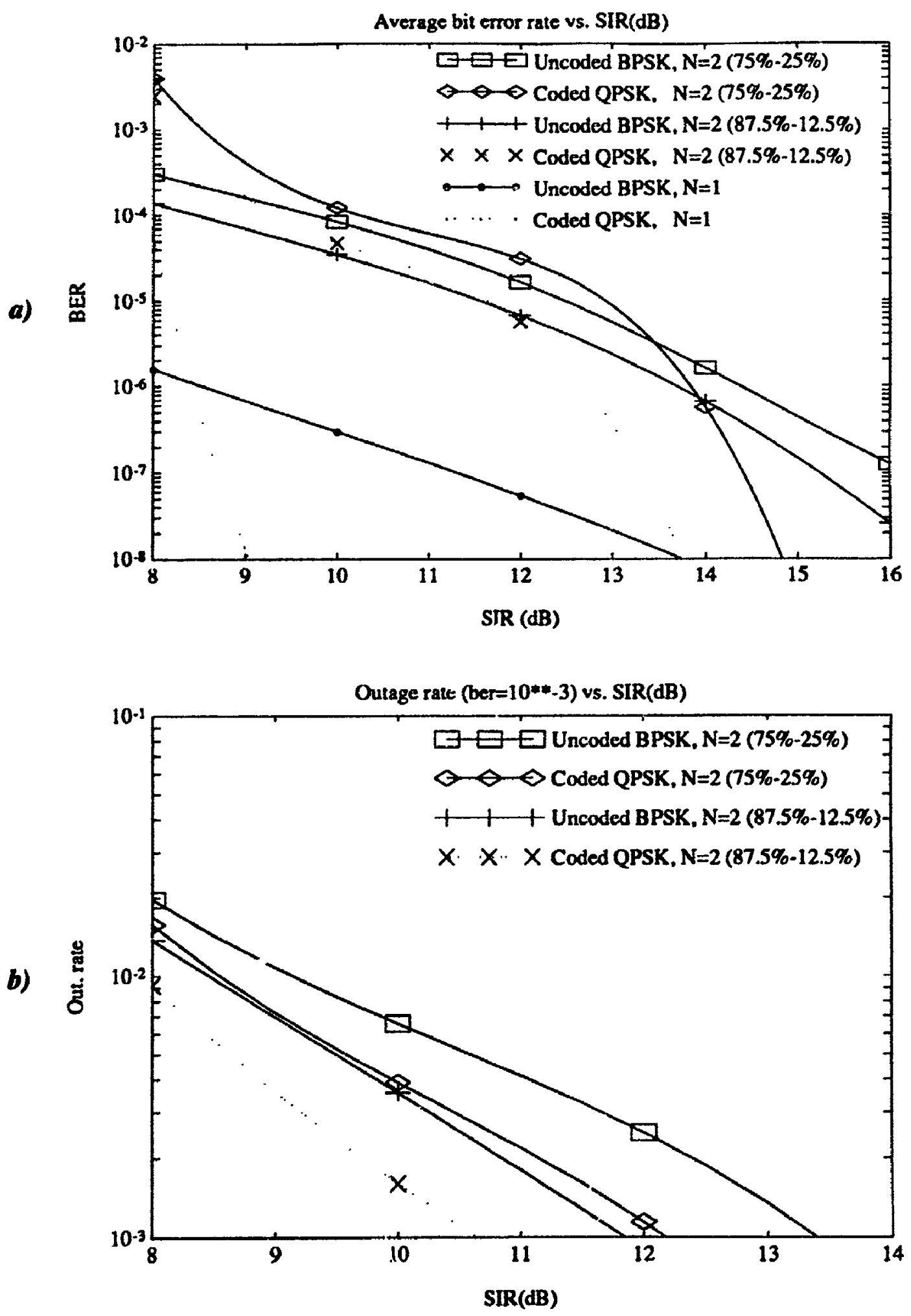

Figure 5.14 Results for $\mathbf{N}=2$ interferers with different power disiributions. $\mathrm{Eb} / \mathrm{N} 0=30 \mathrm{~dB}, \mathrm{M}=2, \mathrm{~L}=3$, cluster channel model (10 Mbits/s data rate), $\mathrm{SRC}$ (50\%) pulse shape. Coded results with no interleaving. $a$ ) Average BER b) Outage rate 

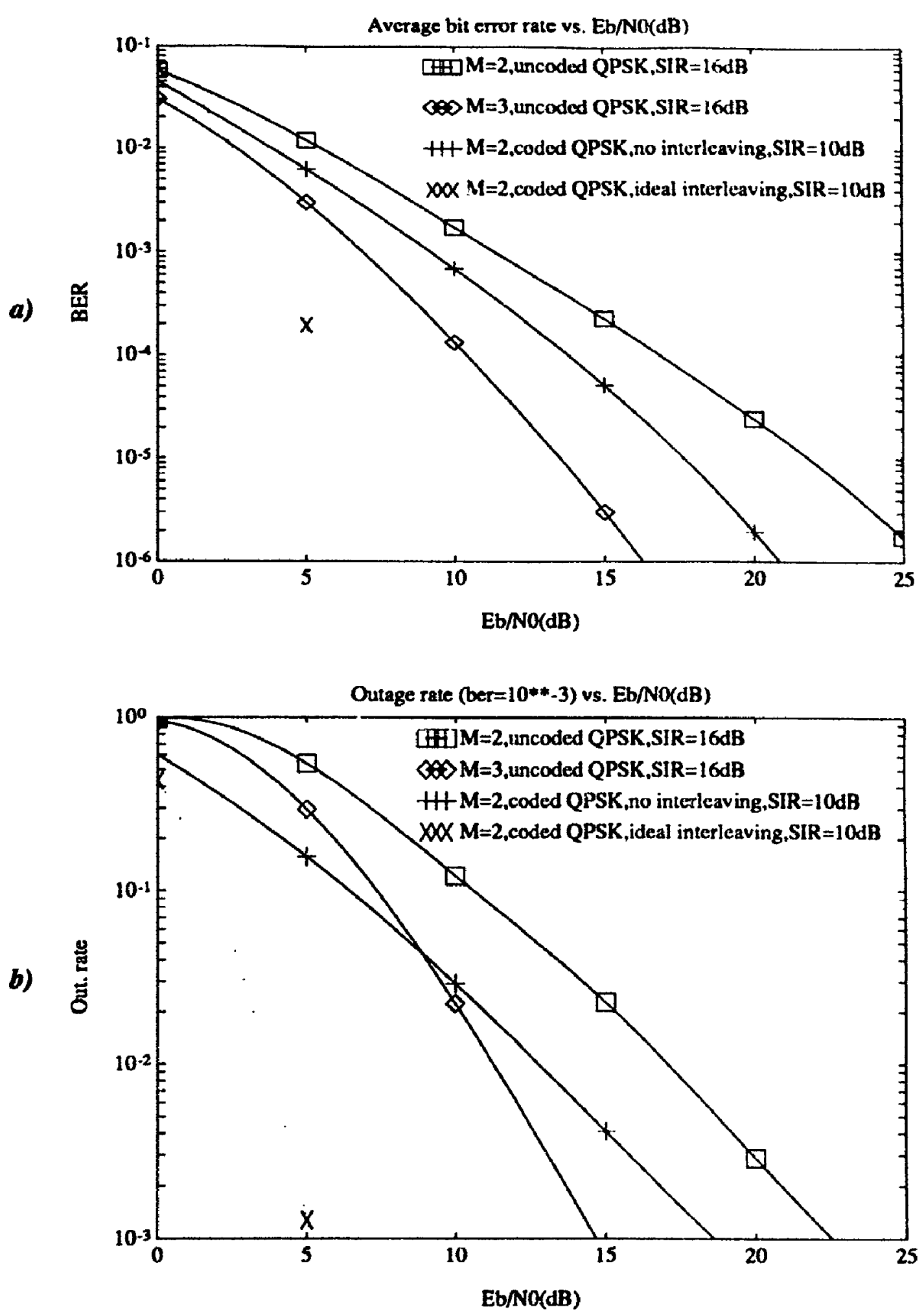

Figure 5.15 Results for $\mathbf{N}=1$ interferer.

L=3, cluster channel model (10 Mbits/s data rate), SRC

(50\%) pulse shape. a) Average BER b) Oufage rate 


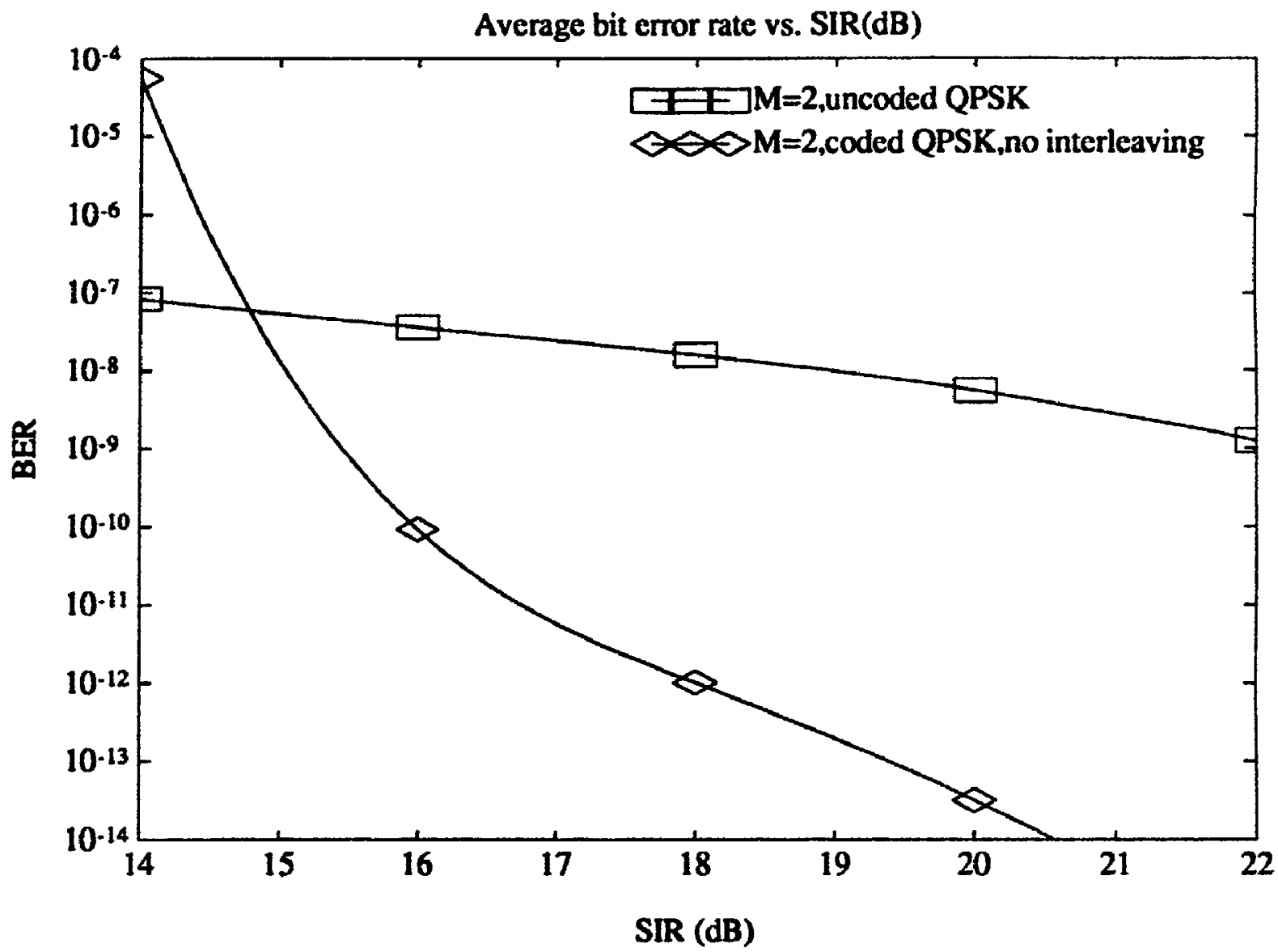

Figure 5.16 Results for $\mathrm{N}=1$ interferer. $\mathrm{Eb} / \mathrm{N} 0=30 \mathrm{~dB}, \mathrm{~L}=3$, cluster channel model (10 Mbits/s data rate), SRC (50\%) pulse shape. Coded QPSK curve is the same as in Figure 5.8 but shifted by $6 \mathrm{~dB}$ to account for bandwidth expansion. 

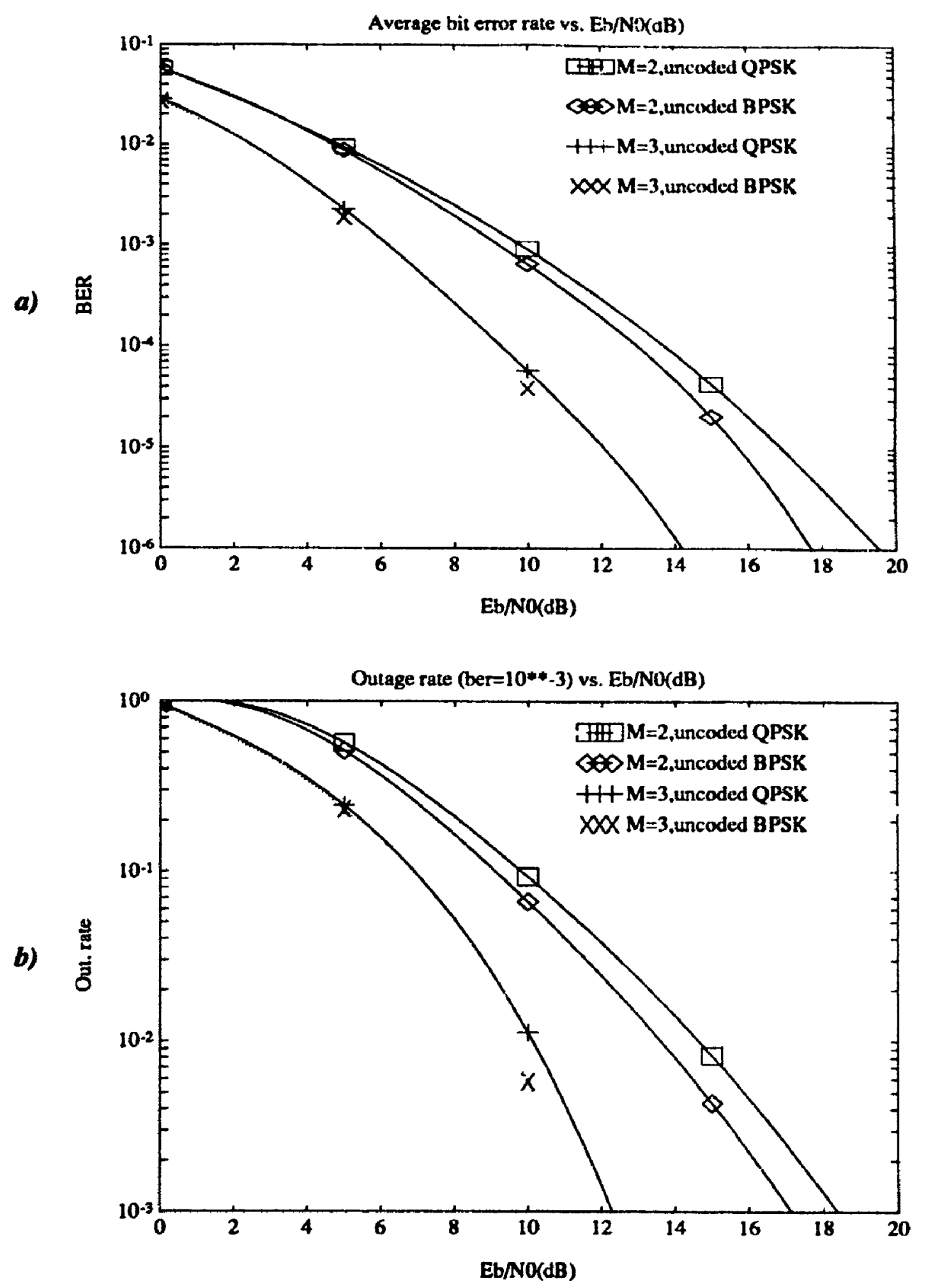

Figure 5.17 BPSK vs. QPSK with $\mathrm{N}=0$ interferer.

L=3, cluster channel model (10 Mbits/s data rate), SRC $(50 \%)$ pulse shape. a) Average BER b) Outage rate 


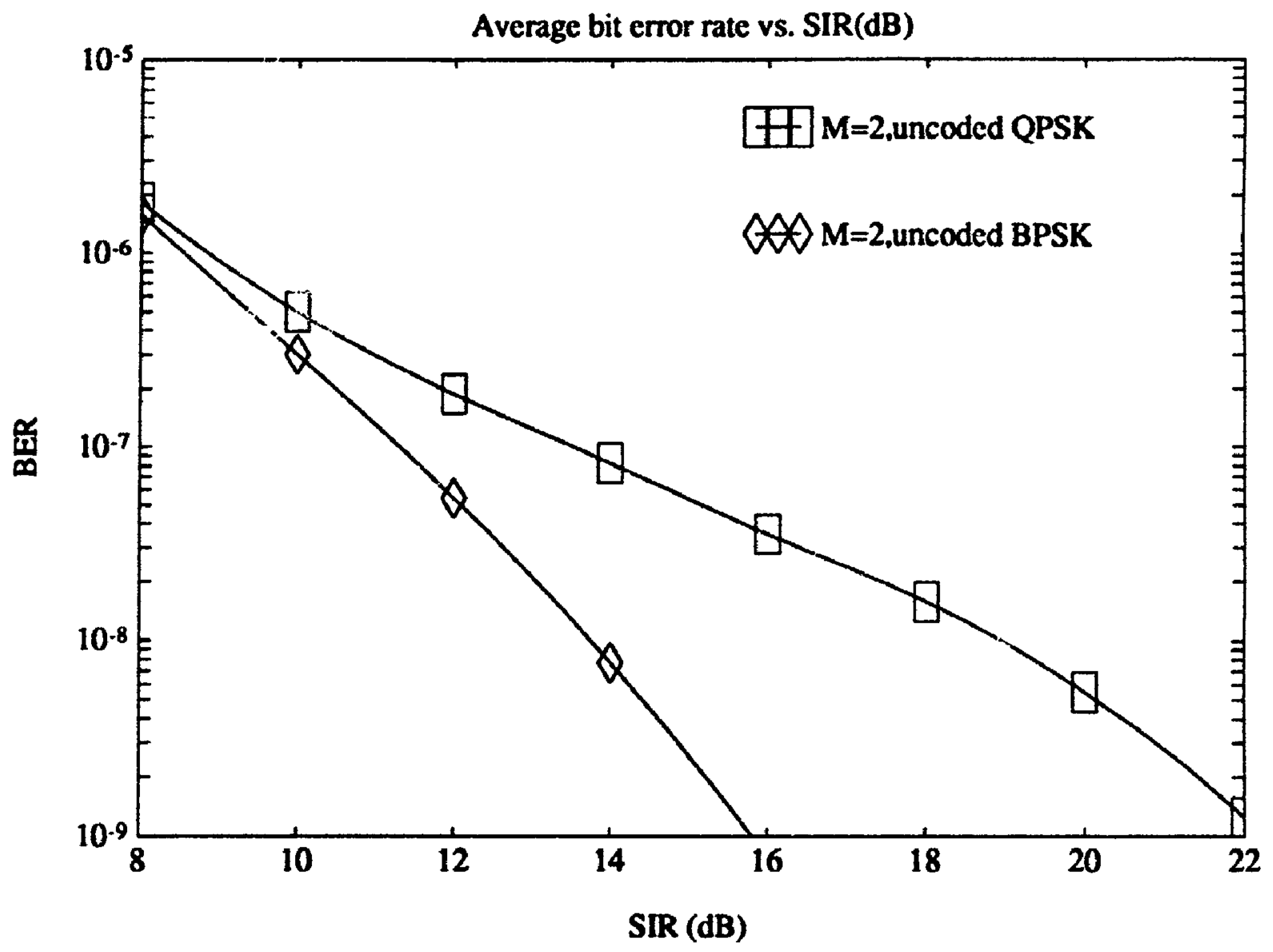

Figure 5.18 BPSK vs. QPSK with $\mathbf{N}=1$ interferer.

$\mathrm{Eb} / \mathrm{N} \rho=30 \mathrm{~dB}, \mathrm{~L}=3$, cluster channel model (10

Mbits/s data rate), SRC (50\%) pulse shape. 

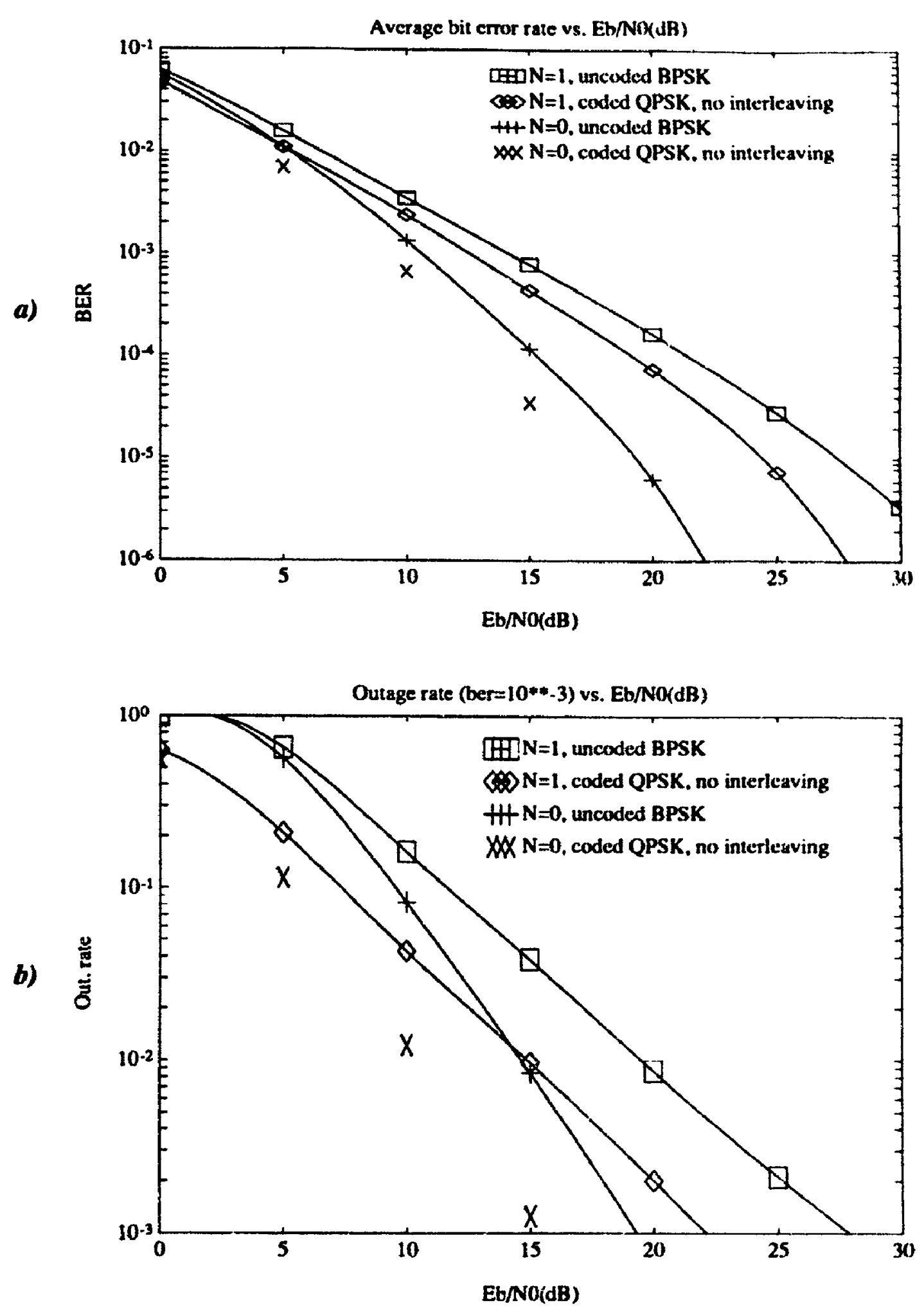

Figure 5.19 Results at a lower data rate with $L=3$ forward filter taps.

$M=2, N=1$, cluster channel model (1 Mbits/s), SRC

(50\%) pulse shape. a) Average BER b) Outage rate 

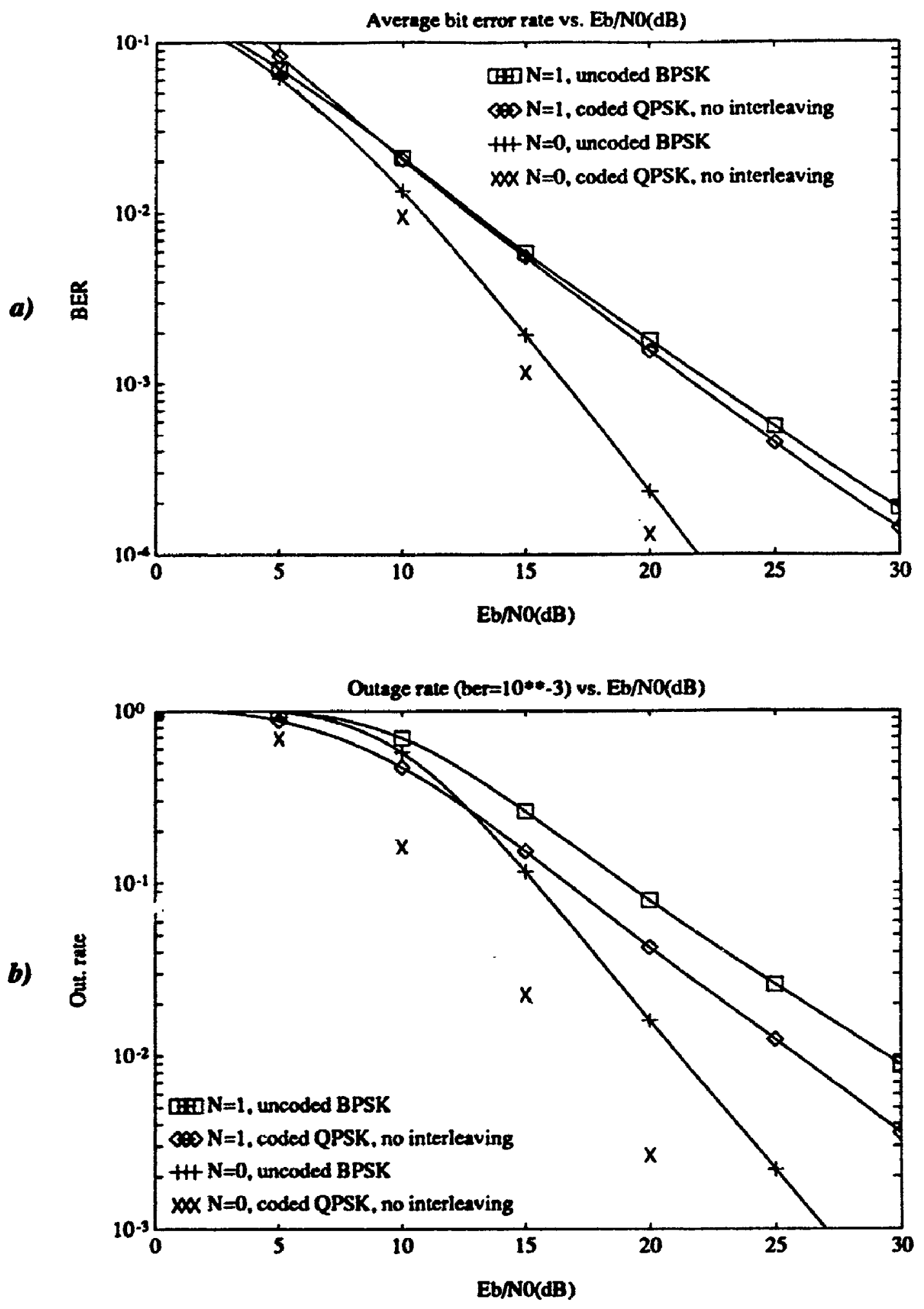

Figure 5.20 Results at a lower data rate with $L=1$ forward filter tap. SIR=10 dB, $M=2, N=1$, cluster channel model (1 Mbits/s), SRC (50\%) pulse shape. a) Average BER b) Outage rate 


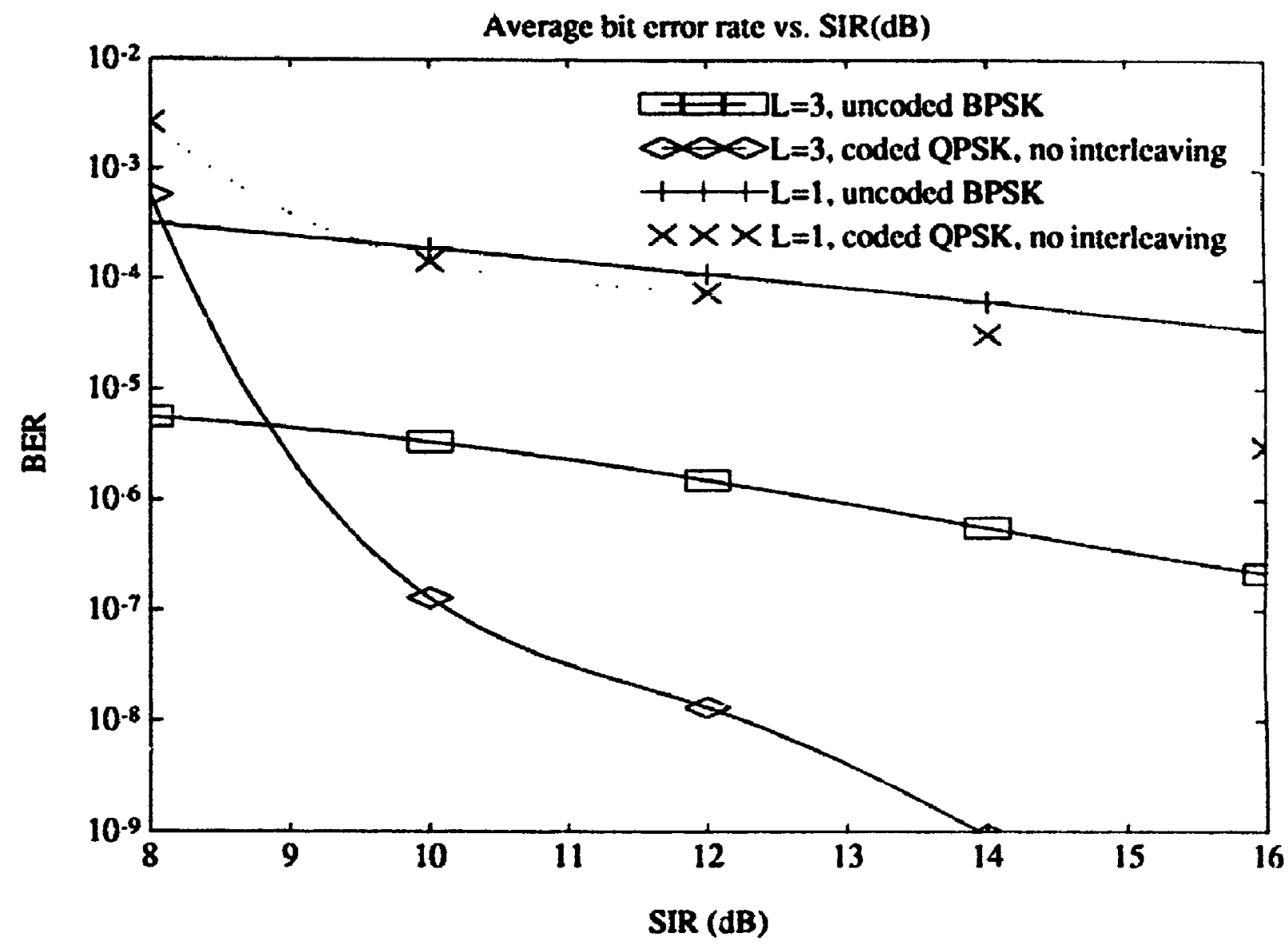

Figure 5.21 Results at a lower data rate with $L=1$ and $L=3$ forward filter taps. $\mathrm{Eb} / \mathrm{NO}=30 \mathrm{~dB}, \mathrm{M}=2, \mathrm{~N}=1$, cluster channel model (1 Mbits/s), SRC (50\%) pulse shape. 

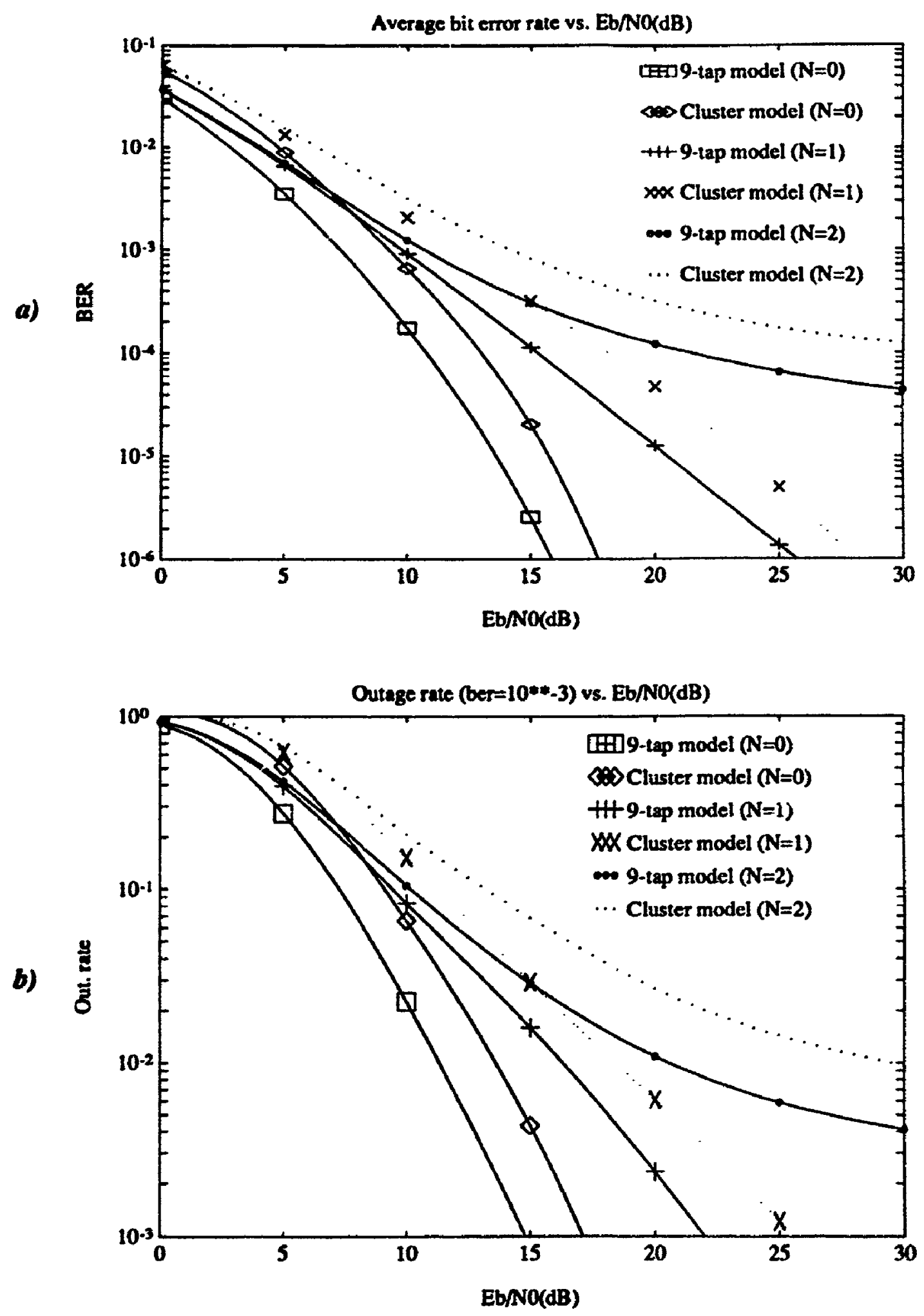

Figure 5.22 Results for different channel models. Equal-power interferers ( $N=2), S_{\text {Lot }}=10 \mathrm{~dB}, M=2, L=3, \sigma_{d}=0.45 T$, BPSK, SRC (50\%) pulse shape. a) Average BER b) Outage rate 


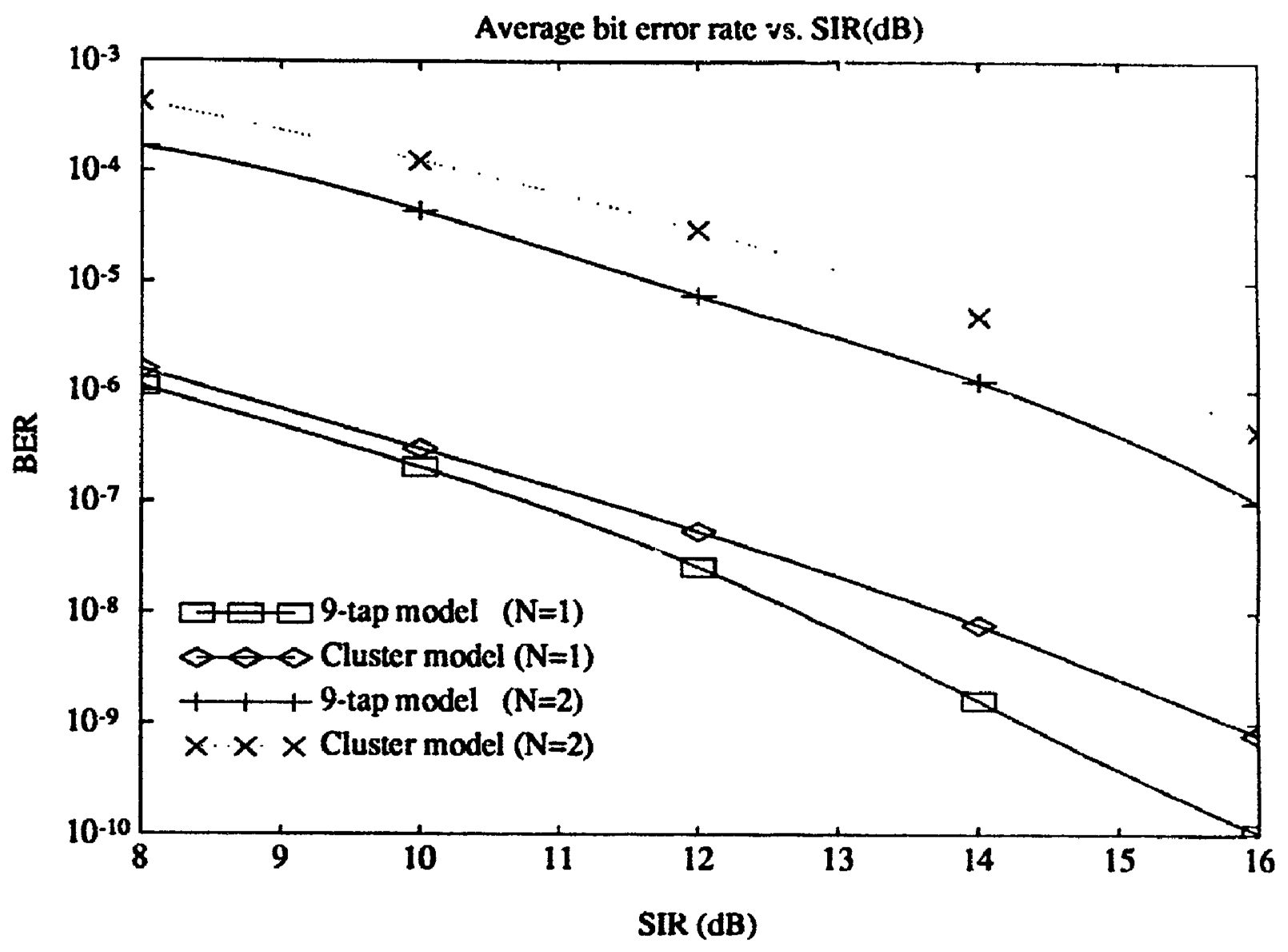

Figure 5.23 Results for different channel models. Equal-power interferers $(\mathrm{N}=2), \mathrm{Eb} / \mathrm{NO}=30 \mathrm{~dB}, \mathrm{M}=2, \mathrm{~L}=3, \sigma_{d}=0.45 T, \mathrm{BPSK}, \mathrm{SRC}(50 \%)$ pulse shape. 

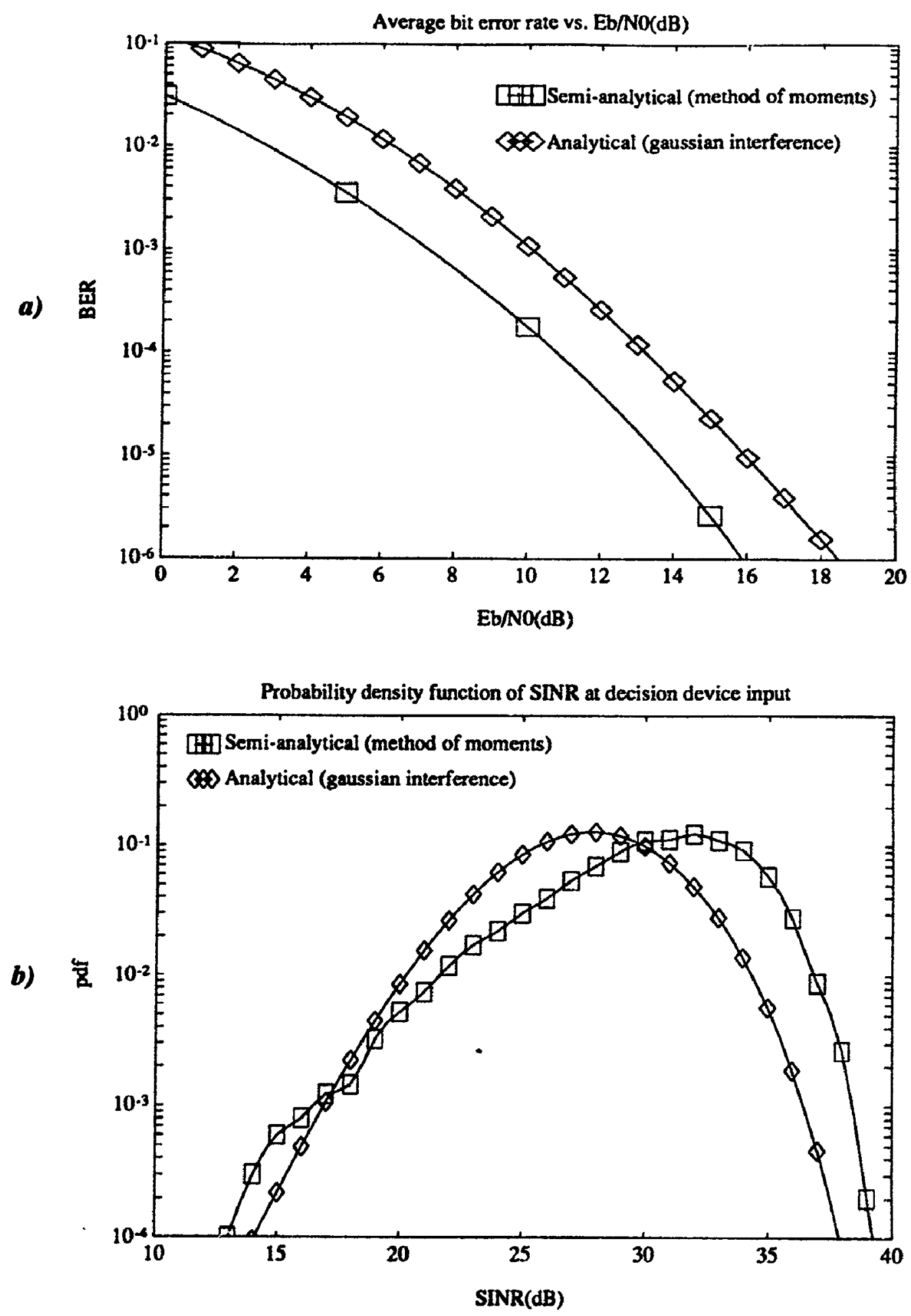

Figure 5.24 Method of moments vs. analysis. $M=2, N=0, L=3, \sigma_{d}=0.45 T$, BPSK, SRC (50\%) pulse shape, 9-tap channel model for method of moments. a) Average BER b) SINR P.D.F.'s at $\mathrm{Eb} / \mathrm{N} 0=30 \mathrm{~dB}$. 


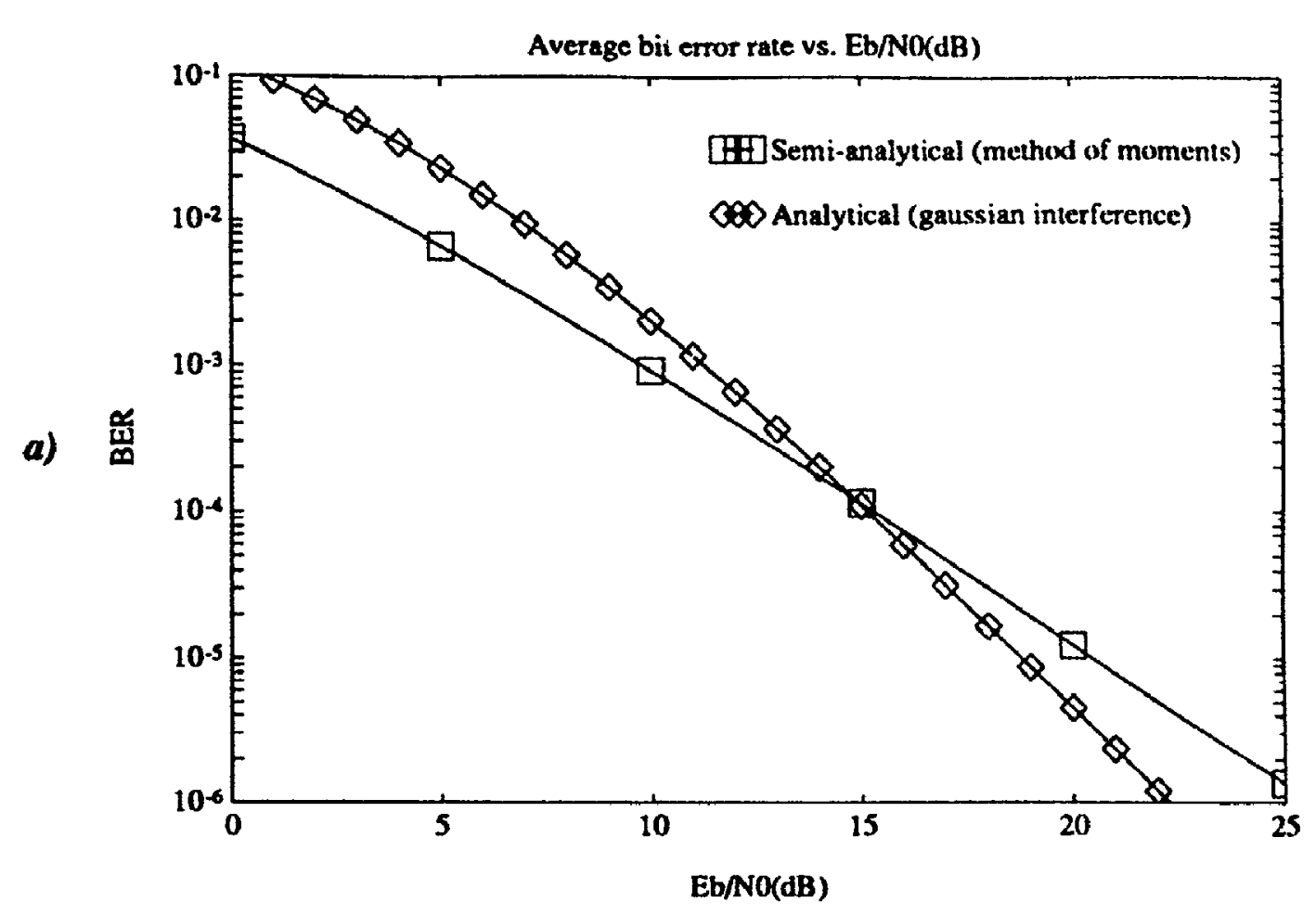

194

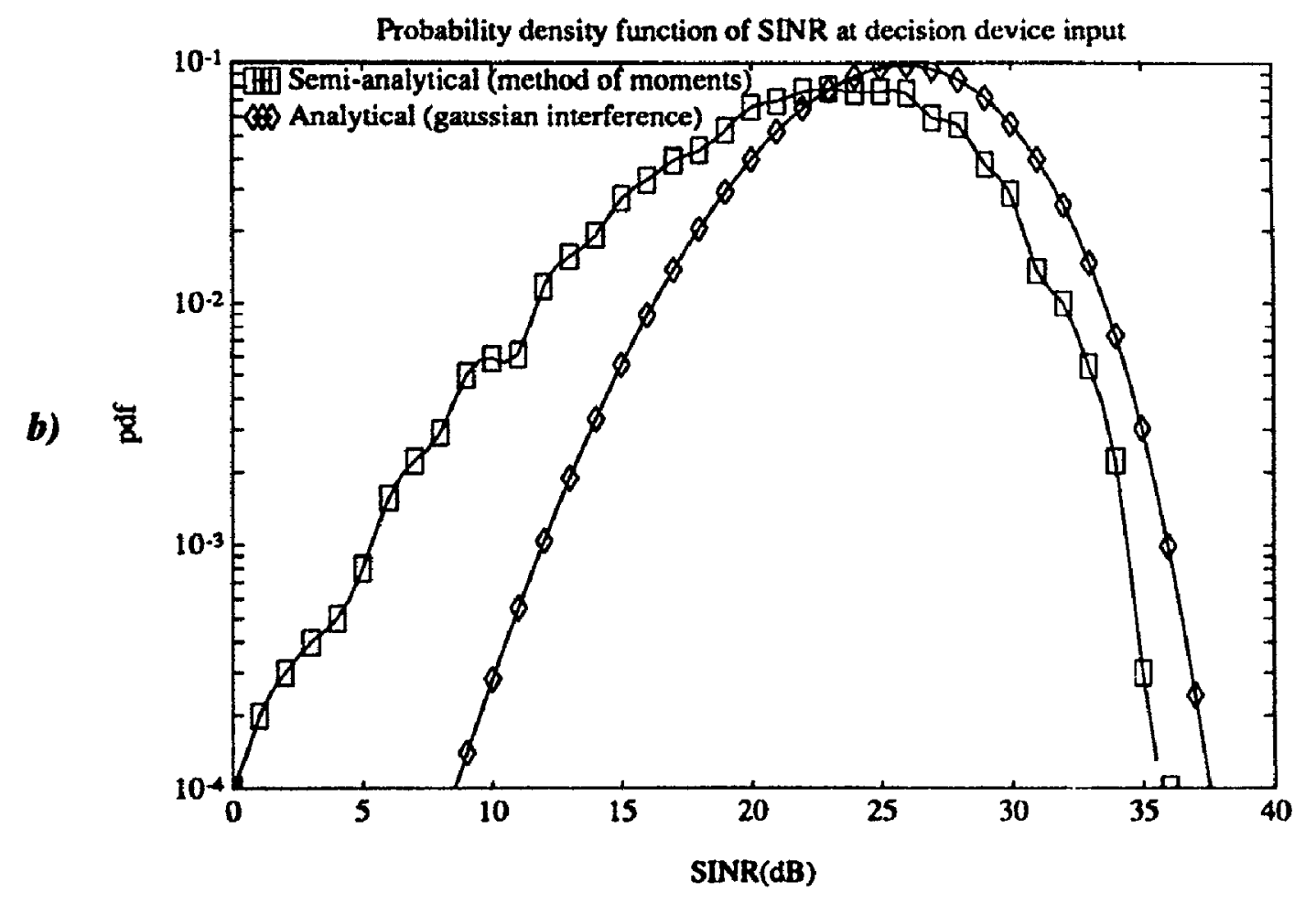

Figure 5.25 Method of moments vs. analysis. $M=2, N=1, S I R=10 \mathrm{~dB}$, $L=3, \sigma_{d}=0.45 T$, BPSK, SRC (50\%) pulse shape, 9-tap channel model for method of moments. a) Average BER b) SINR P.D.F.'s at Eb/N0=30dR. 

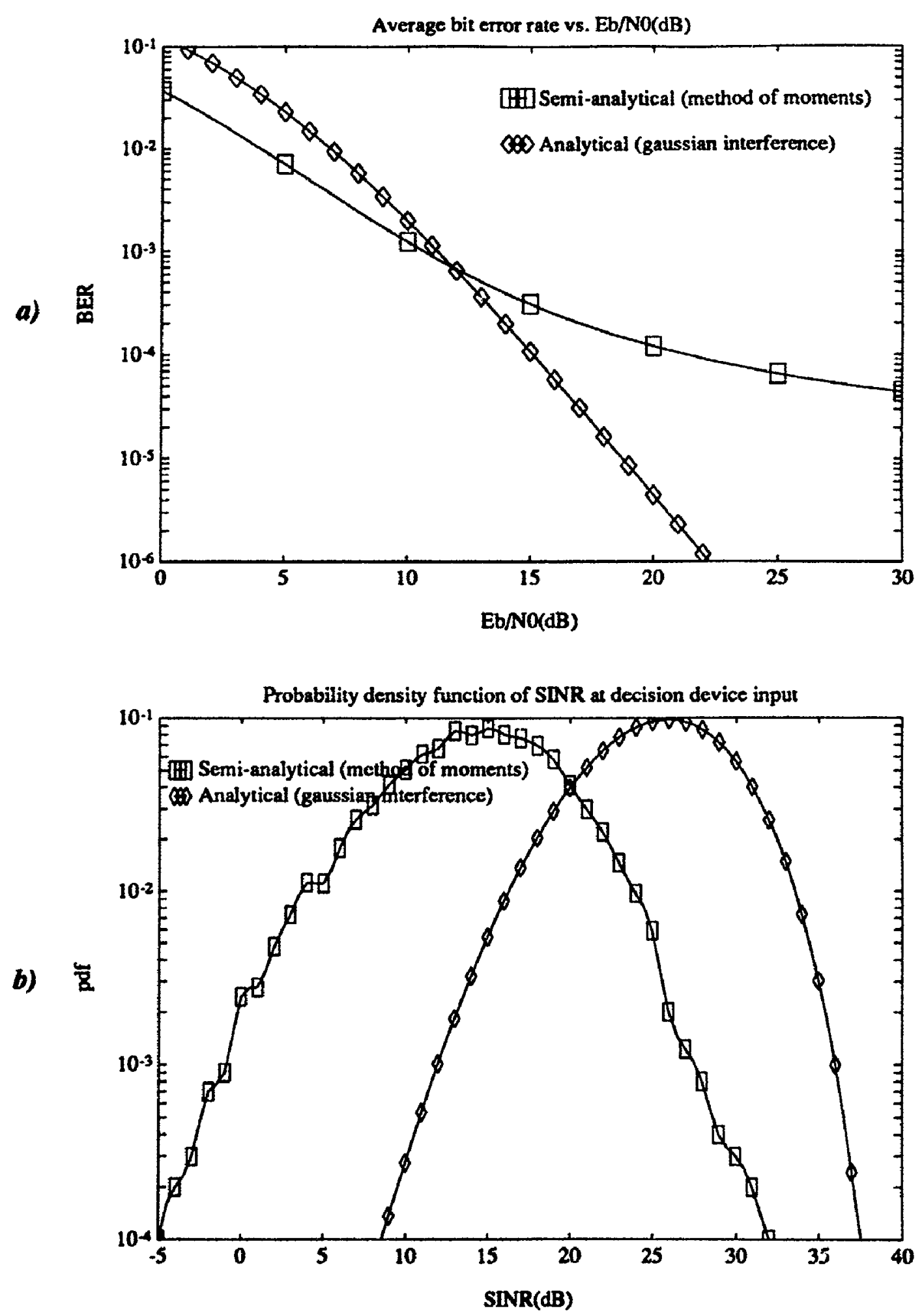

Figure 5.26 Method of moments vs. analysis. $M=2, N=2, S_{\text {tot }}=10 \mathrm{~dB}$, $L=3, \sigma_{d}=0.45 T$, BPSK, SRC (50\%) pulse shape, 9-tap channel model for method of moments. a) Average BER b) SINR P.D.F.'s at Eb/N0=30dB. 


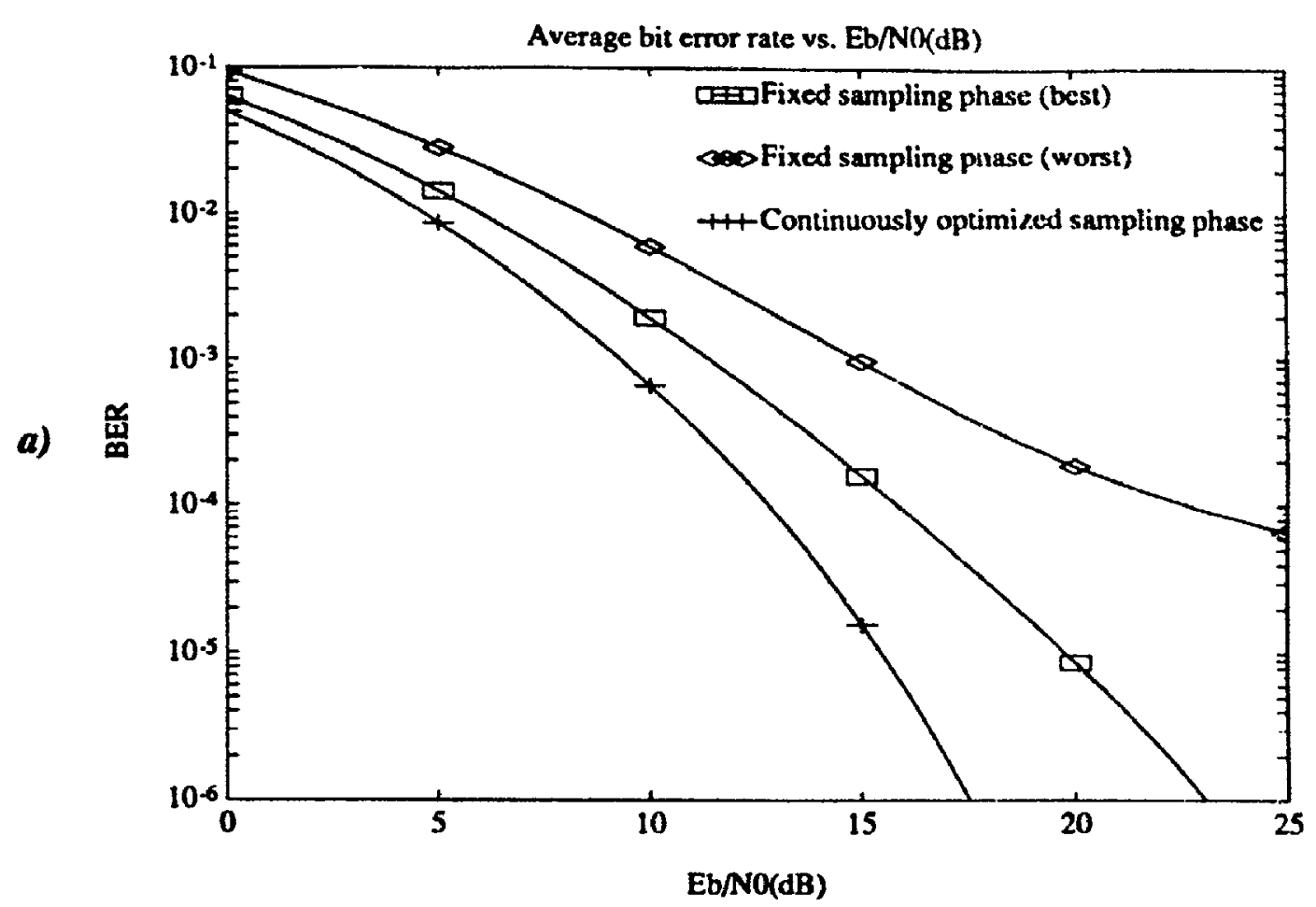

196

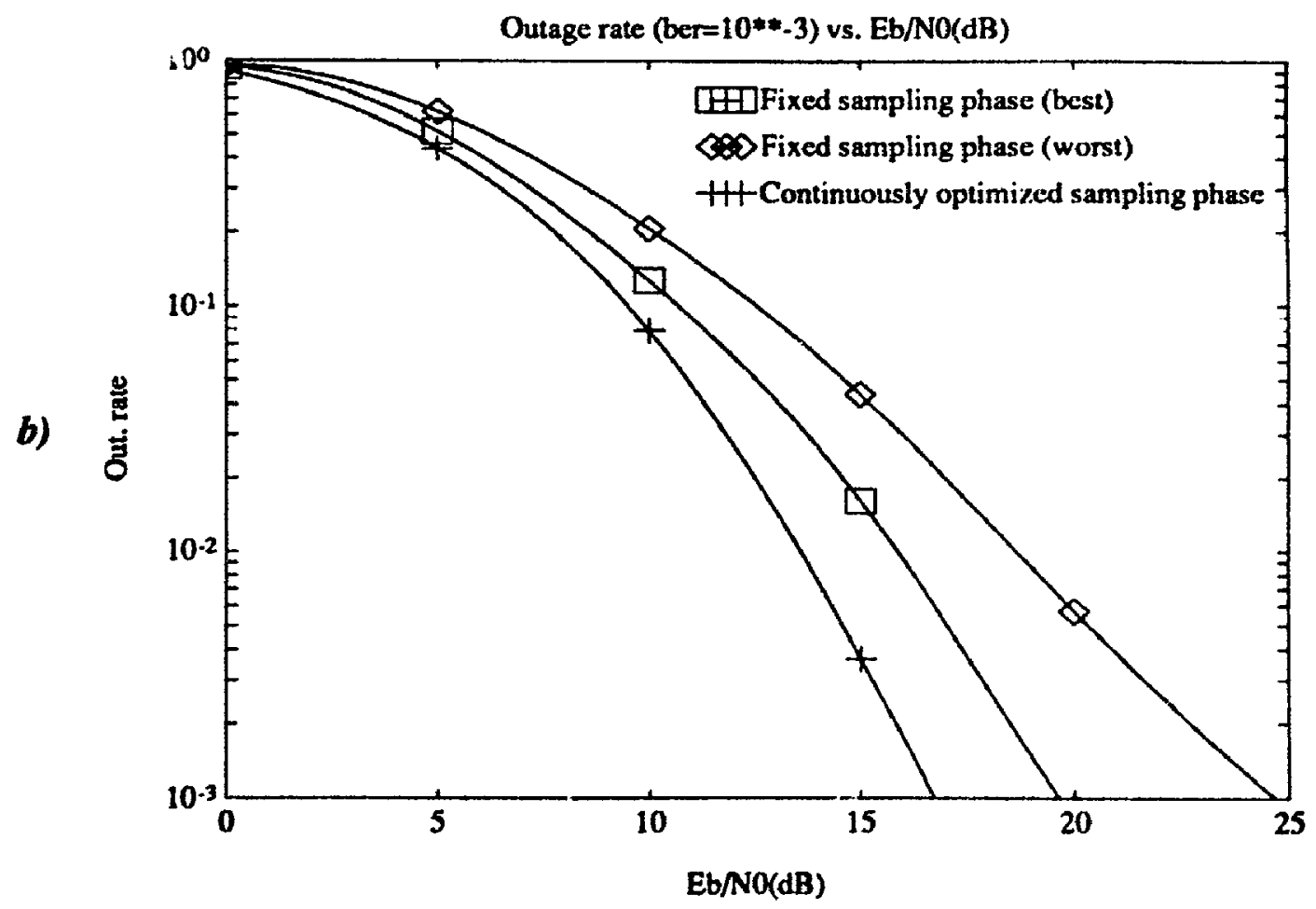

Figure 5.27 Fixed vs. continuously optimized sampling phase.

$M=1, N=0, L=3, B P S K$, 9-tap channel model, $\sigma_{d}=0.25 T$, time-rectangular pulse shape. a) Average BER b) Outage rate 

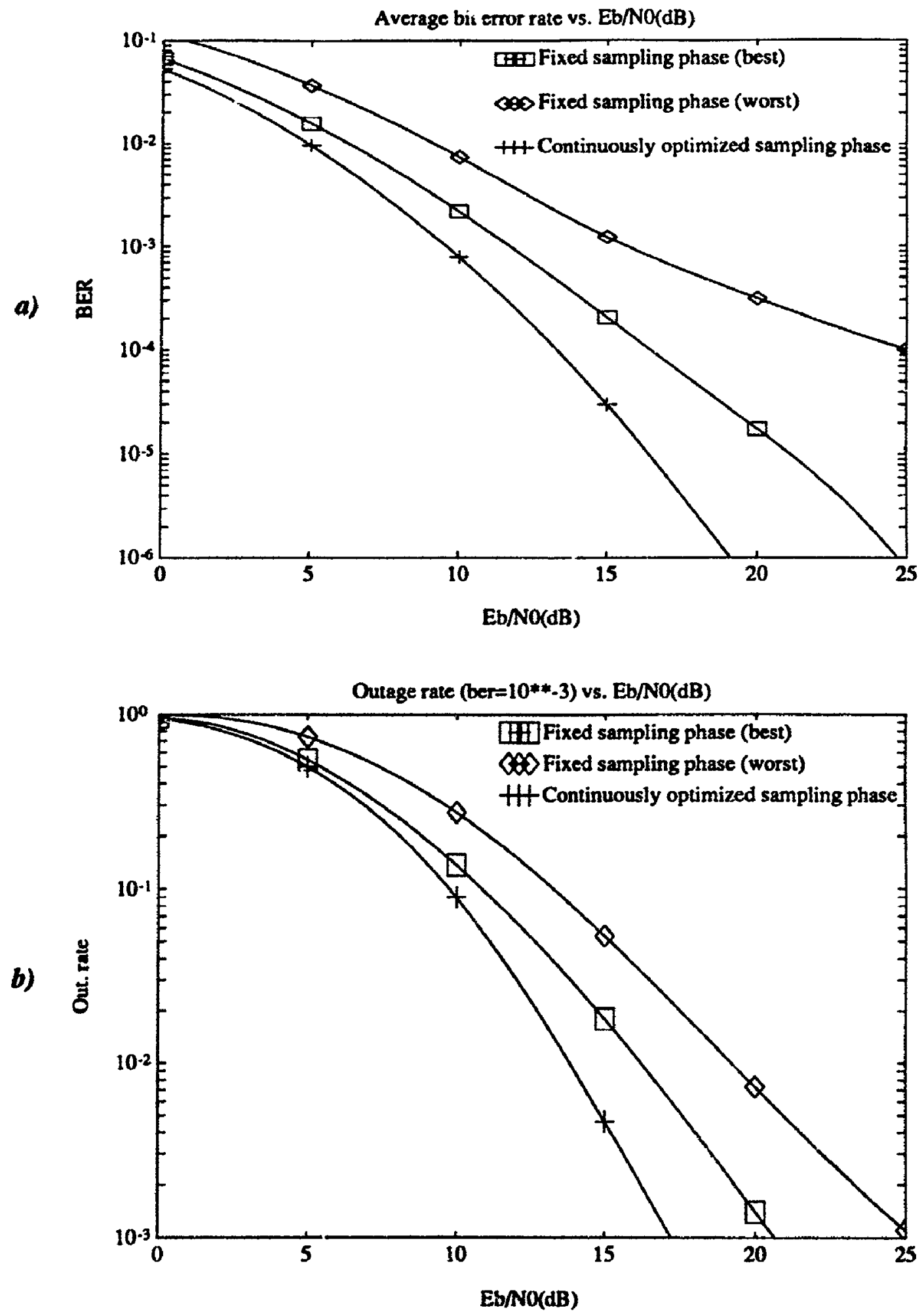

Figure 5.28 Fixed vs. continuously optimized sampling phase.

$M=1, N=0, L=3, B P S K, 9-t a p$ channel model, $\sigma_{d}=0.50 T$, time-rectangular pulse shape. a) Average BER b) Outage rate 

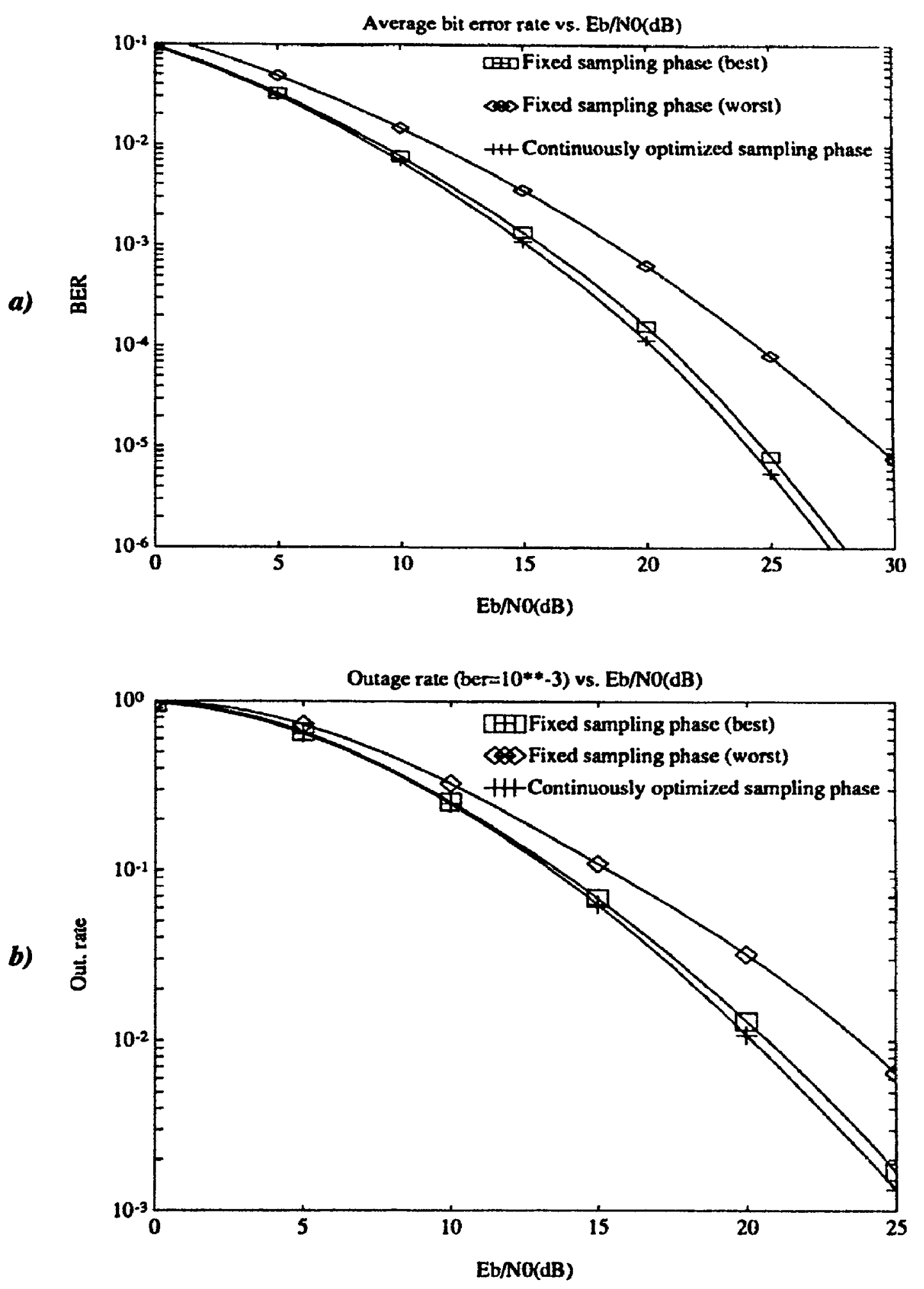

Figure 5.29 Fixed vs. continuously optimized sampling phase.

$M=1, N=0, L=3, B P S K$, 9-tap channel model, $\sigma_{d}=0.25 T$, SRC (50\%) pulse shape. a) Average BER b) Outage rate 

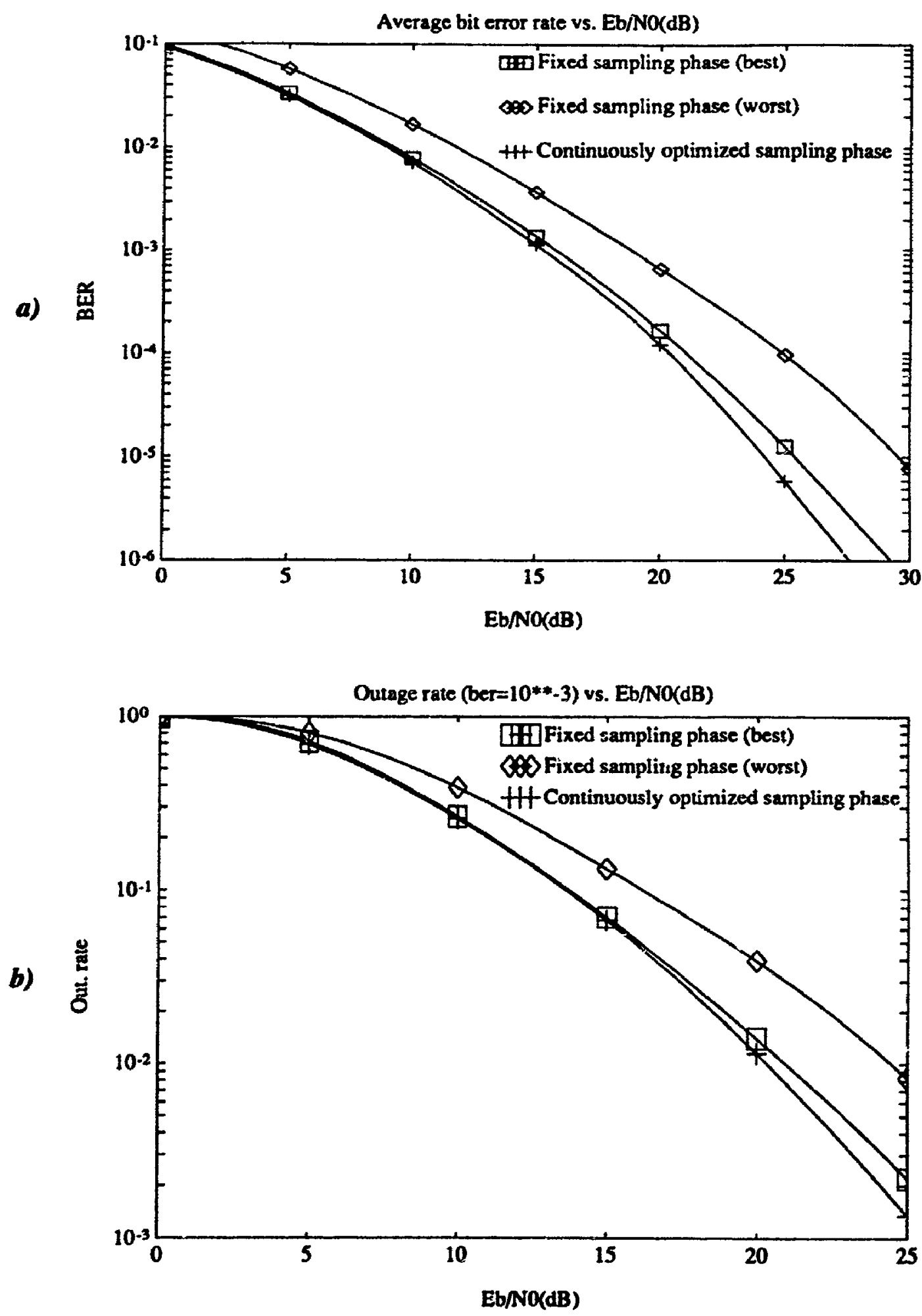

Figure 5.30 Fixed vs. continuously optimized sampling phase.

$M=1, N=0, L=3, B P S K$, 9-tap channel model, $\sigma_{d}=0.50 T$, SRC (50\%) pulse shape. a) Average BER b) Outage ? ate 


\subsection{Summary}

In this Chapter, the performance of optimum baseband combining in conjunction with channel coding on a quasi-static indoor fading channel has been examined using a semi-analytical approach. The latter is based on static channel analytical performance evaluation via the method of moments and Monte Carlo averaging over the fading channel statistics. Expressions have been derived for the moment-generating function of the decision variate in the uncoded case and in coded cases with both ideal interleaving and no interleaving. In addition to providing results for the uncoded case and these two coded cases, such a technique eliminates the non-fading gaussian noise characterization of interference inherent in the purely analytical approach of Chapter 3 while producing statistically significant results much faster than the simulation described in Chapter 4. Some of the main conclusions are:

1. Based on a realistic cluster channel model and on a data rate of $10 \mathrm{Mbits} / \mathrm{s}$, the use of a simple, short constraint length rate $\frac{1}{2}$ convolutional code with QPSK modulation has been shown, even in the absence of interleaving, to be a viable alternative to increasing the explicit space diversity order of a dual space diversity optimum baseband combiner employing BPSK and with three taps per forward filter. However, with an SIR of $10 \mathrm{~dB}$, this conclusion only holds in the presence of a single cochannel interferer. In this case, the dual antenna coded, non-interleaved system can recuperate $50 \%$ of the average BER gain and $80 \%$ of the outage rate gain obtained by adding a third space diversity branch. In the presence of two cochannel interferers with a total SIR of $10 \mathrm{~dB}$, the uncoded three-antenna system is clearly preferable to the two-antenna, coded, non-interleaved scheme for which large irreducible average bit error rates and outage rates are then observed; e.g. an outage rate of $10^{-3}$ does not seem attainable unless the lower power interferer is at least $10 \mathrm{~dB}$ below the most powerful interferer. On the other hand, if the total SIR with two interferers is made larger than $15 \mathrm{~dB}$, it appears that the dual antenna, coded, non-interleaved scheme could again become a viable alternative to increasing the uncoded system's space diversity order. It should also be noted 
that, based on an uncoded QPSK to rate $\frac{1}{2}$ coded QPSK comparison, coding with no interleaving can still be attractive but the resulting bandwidth expansion will increase the aforementioned SIR thresholds by up to $6 \mathrm{~dB}$.

2. As the implicit diversity effect becomes less significant at lower data rates, coding with no interleaving is then not as attractive. At a data rate of $1 \mathrm{Mbits} / \mathrm{s}$, coding gains are smaller than at $10 \mathrm{Mbits} / \mathrm{s}$ but the highly variable nature of the cluster channel model's impulse response realizations implies that some benefits of implicit diversity are still obtained at 1 Mbits/s.

3. In general, coding with no interleaving tends to be more attractive (as opposed to average BER) in terms of outage rate which is really the criterion of interest for portable communications.

4. At a data rate of $10 \mathrm{Mbits} / \mathrm{s}$, performance predictions based on simple, exponential delay profile, nine-tap channel model tend to be optimistic with respect to those obtained with the more realistic cluster model of equal ms delay spread. This results again from the highly variable nature of the cluster model responses whose duration, as opposed to the fixed duration of the nine-tap model responses, may often significantly exceed the equalizer span.

5. The non-fading gaussian characterization of interference in the purely analytical performance evaluation of Chapter 3 limits its use to cases where the number of interferers is very small (one and possibly two) and where the total signal-tointerference ratio is large.

6. With $M=1$ antenna and no cochannel interference, continuously optimizing the receiver sampling phase has been shown to prevent $3 \mathrm{~dB}$ to $4 \mathrm{~dB}$ performance degradations which could result from the selection of an improper fixed sampling phase when the rms delay spread is the $0.25 \mathrm{~T}$ to $0.50 \mathrm{~T}$ range. With coding or in the presence of cochannel interference, these variations could be larger but they also can be alleviated with more forward filter taps or explicit space diversity branches. 


\section{Chapter 6}

\section{CONCLUSION}

\subsection{Thesis summary}

This research was motivated by the quest to achieve universal portable and personal communications. In order to realize this vision, a low-power tetherless access system must be designed to complement current and future vehicular radio, mobile satellite as well as wireline networks in such a way that all of these can be integrated into a unified access architecture. For indoor wireless communications, two different channel access schemes, FD-TDMA and CDMA, are currently the focus of extensive research and this thesis has contributed to this effort by examining the TDMA option. In particular, a wideband TDMA access mode is attractive in order to enhance system versatility and flexibility in an environment where data rates of up to $20 \mathrm{Mbits} / \mathrm{s}$ are predicted [23] for future applications.

The dominant impairments in the wideband TDMA indoor channel are typically shadowing, slow frequency-selective fading and cochannel interference. With the aid of macroscopic diversity to alleviate the effects of shadowing, a receiver structure which conjugates post-detection space diversity and equalization into a scheme with multiple forward filters and possibly a common feedback filter is then an attractive technique to simultaneously combat signal fading, intersymbol interference ard cochannel interference on the radio links between the portables and the base stations. Although compact, built-in dual diversity antennas with good decorrelation characteristics have been developed for the portables, the extension to the three-antenna case appears difficult considering the small size of these hand-held units. In view of current advance.; in VLSI technology, the main objective of this thesis has thus involved determining if the use of a simple, short constraint length convolutional code with soft-decision Viterbi decoding could be a viable alternative to increasing from two to three the space diversity order of such an optimum (or MMSE) baseband combiner employing coherent 
BPSK or QPSK modulation. Following a detailed teview of the portable indoor channel's characteristics and a survey of the various countermeasures available to mitigate the channel's impairments, this thesis has presented a three-fold approach to assessing the coding vs. diversity performance trade-offs on these wideband TDMA indoor radio links employing optimum baseband combining.

\subsubsection{Analytical approach}

A purely analytical approach was initially described in Chapter 3. In the process, previous (uncoded) optimum baseband combiner derivations for tropospheric scatter channels [50] were applied to frequency-selective, Rayleigh fading, indoor wireless radio links employing both BPSK and QPSK modulations and novel average BER bounds were developed for the corresponding case with convolutional coding and softdecision Viterbi decoding. Such a technique is advantageous as it quickly yields a large number of results without resorting to lengthy simulations. However, it is based on a non-fading, gaussian characterization of interference which is not realistic as cochannel interference is most often dominated by a single source; this tends to restrict the relevance of the results to cases where the error rate is affected more by fading of the desired signal than by intersymbol and cochannel interference. In the coded case, this analytical technique is also based on an assumption of ideal interleaving which, for the portable application, is difficult to realize both in terms of delay constraints (for speech) and complexity (for data rates in the Mbits/s range). The coded results are thus optimistic and represent a lower bound on achievable performance. Based on an exponential delay profile channel model, some of the main conclusions are:

1. With $L=3 \frac{T}{2}$-spaced forward filter taps and a rms delay spread $\sigma_{d}=0.25 T$, the ability of an increased explicit space diversity order $M$ to reduce sampling phase sensitivity and to minimize its increase when cochannel interference is added is stronger for a smaller excess bandwidth pulse.

2. For a given fixed receiver sampling phase, $M=2$ antennas and $\sigma_{d}=0.25 T$, increasing the number of forward filter taps $L$ beyond 5 yields only a negligible performance improvement. More than $90 \%$ of the improvement observed from 
$\mathrm{L}=1$ to $\mathrm{L}=5$ is obtained with $\mathrm{L}=3$.

3. With $L=3$ taps, a rms delay spread of at least $\sigma_{d}=0.10 T$ is required to significantly exploit the implicit diversity of the channel for both the $M=2$ and $M=3$ cases. No significant performance degradation then occurs until $\sigma_{\mathrm{d}}$ exceeds 0.50T.

4. The $M=2$ coded system can tolerate lower $\frac{S}{T}$ values than the uncoded $M=2$ and $M=3$ systems before a non-negligible performance degradation occurs. However at very low $\frac{S}{T}$, coded system performance can degrade suddenly.

5. The transition point in terms of data rate beyond which QPSK is superior to BPSK is higher when interference dominates than when AWGN dominates. It is also higher with smaller $M$ when cochannel interference is significant. If cochannel interference is negligible, the transition point is similar for $M=2$ and $M=3$.

\subsubsection{Bit-by-bit simulation}

In Chapter 4, a bit-by-bit simulation approach was then described where the effect of the non-interleaved channel was assessed with all analytical assumptions relaxed. However, in view of execution time constraints, the quasi-static nature of the channel precluded the generation of statistically significant results. Following a confirmation of the cyclostationary nature of the sum of two received cochannel interferers, a simulation with $L=3$ forward filter taps, $N=1$ cochannel interferer with $\frac{S}{T}=10 d B$ and a channel with an exponential delay profile and a rms delay spread $\sigma_{d}=0.25 T$, showed that the coded system was more dependent on sampling phase than the uncoded system. Given the proper fixed sampling phase, a larger number of taps or a continuously updated sampling phase, it was thus shown that using convolutional coding with soft-decision Viterbi decoding and no interleaving, could be a valid alternative, particularly in terms of outage rate, to increasing the space diversity order from $M=2$ to $M=3$. In fact, the on-off behavior of the coded results also lead to an outage improvement for a suboptimal sampling phase even if the corresponding average BER was much worse than for the uncoded cases.

\subsubsection{Semi-analytical approz $h$}

In Chapter 5, performance was assessed using a semi-analytical approach based 
on static channel analytical performance evaluation via the method of moments and Monte Carlo averaging over the fading channel statistics. Expressions were derived for the moment-generating function of the decision variate in the uncoded case and in coded cases with both iderl interleaving and no interleaving. In addition to providing results for the uncoded case and these two coded cases, such a technique eliminated the non-fading gaussian noise characterization of interference inherent in the purely analytical approach of Chapter 3 while producing statistically significant results much faster than the simulation described in Chapter 4. The main findings were:

1. Based on a realistic cluster channel model and on a data rate of $10 \mathrm{Mbits} / \mathrm{s}$, the use of a simple, short constraint length rate $\frac{1}{2}$ convolutional code with QPSK was again found to be a viable altemative, even in the absence of interleaving, to increasing from two to three the explicit space diversity order of a three forward filter tap optimum baseband combiner with BPSK modulation. However, with a SIR of 10 dB, this conclusion only holds in the presence of a single cochannel interferer. With two cochannel interferers, coding only appears to be attractive for a SIR greater than $15 \mathrm{~dB}$. If the coding and diversity are compared on the basis of rate $\frac{1}{2}$ coded QPSK vs. uncoded QPSK, the bandwidth expansion resulting from coding increases the aforementioned SIR thresholds by about $6 \mathrm{~dB}$.

2. With the cluster channel model and three $\frac{T}{2}$-spaced taps, some benefits of implicit diversity are still obtained at a data rate of 1 Mbits/s.

3. Coding with no interleaving is more attractive in terms of outage rate than in terms of average BER.

4. The limitations of the purely analytical approach of Chapter 3 were demonstrated. The assumed non-fading nature of interference is its most serious drawback as the mean-square value of the gaussian interfering symbols can always be adjusted to yield relevant results for a given set of system parameters.

5. For the simple case of $M=1$ antenna with $L=3$ taps and no cochannel interference, a continuous update of sampling phase has been shown to prevent degradations of up to $4 \mathrm{~dB}$ which could result from the selection of a suboptimal fixed sampling phase. 
It is thus siear that coding with no interleaving is of no use unless diversity is also used in tandem to alleviate the effects of fading and interference; in view of the on-off behavior of coded systems, coding then becomes attractive if the diversity order is sufficiently large to raise the SINR above a certain threshold below which corled performance deteriorates very quickly. As was found in [56], when comparing a non-interleaved coded system with diversity order $M$ to an uncoded system with diversity order $M+1$, coding is clearly more viable for larger $M$. However, exploiting the channel's implicit diversity effect via the $L=3$ forward filter taps has now raised the effective diversity order and thus improved the attractiveness of a simple noninterleaved code even with a relatively small explicit diversity order of $M=2$. In this sense, the coded scheme becomes more attractive as the signaling rate increases from the flat fading case. On the other hand, channel coding with no interleaving cannot match the performance obtained by adding a third diversity branch and the SIR required to obtain positive coding gains can be quite large.

Given the fact that one cochannel interferer most often dominates and that the number of significant cochannel interferers should thus rarely exceed two, the coded, non-interleaved optimum baseband combiner with $M=3$ should be a far better alternative to the corresponding $M=4$ uncoded system than the $M=2$ coded system was found to be compared to the $\mathrm{M}=3$ uncoded system. On the radio links, a desirable design approach thus consists of applying as much (explicit and implicit) diversity as possible in view of complexity and size constraints and if the resulting diversity order is sufficiently large, coding even without interleaving can be used to further improve performance. The macroscopic diversity scheme must then be designed to maintain the mean SIR on the links above certain level; e.g. with two interferers, the $M=2$, coded, non-interleaved system appeared to be viable for a total SIR larger than $15 \mathrm{~dB}$ while with a single interferer, a SIR of only $10 \mathrm{~dB}$ is sufficient. It is reasonable to speculate that the latter would probably be sufficient to make a $M=3$, coded, non-interleaved system viable in the presence of two interferers. It should also be clear that, with only three $\frac{T}{2}-$ spaced forward filter taps, some form of continuous sampling phase optimization is required to avoid degradations which could be particularly severe with the coded system. 


\subsection{Recommendations for further research}

The findings of this thesis indicate the need for further research in the following areas.

\subsubsection{Influence of diversity vs. coding trade-off on cochannel reuse ratio}

This thesis has dealt almost exclusively with radio link performance and macroscopic system issues have not been addressed. As briefly discussed in Chapter 5, the cochannel reuse ratio $\frac{D}{R}$, i.e. the ratio of the distance $D$ between the centers of nearest neighboring cochannel cells to the cell radius $R$, is a key parameter in the design of any high capacity cellular system. This $\frac{D}{R}$ ratio has an impact on both the transmission quality and the ultimate customer capacity of the system. Making $\frac{D}{R}$ as small as possible serves the objective of large capacity while making it as large as possible benefits transmission quality by increasing the mean received SIR. It would be thus be of interest to translate the results of this thesis into improvements in the minimum $\frac{D}{R}$ required ${ }^{82}$ to achieve a given performance specification, typically in terms of outage rate. Such a study was recently performed [213] for both block and convolutional codes under the assumption of ideal interleaving in flat fading with a fixed diversity order. A few brief results are also given in [213] for the case of no interleaving but again in flat fading and with a fixed diversity order. This case of no interleaving should thus be thoroughly investigated for different explicit space diversity orders and different combining schemes as well as for the frequency-selective fading case where the channel's implicit diversity effect can be exploited to 'mprove performance. It would also be of interest to obtain results in terms of cellular efficiency, i.e. the number of users/cell/MHz, as in [214] where again ideal interleaving is assumed.

\subsubsection{Performance evaluation with partial interleaving}

As the case of no interleaving represents the most pessimistic situation in terms of code performance, it would be worthwhile to also evaluate radio link performance on

$82 \quad \frac{D}{A}$ can take on only a finite set of values as, e.g. for a hexagonal cellular geometry, it is related to the number of cells $N_{c}$, i.e. channel sets out of the total bandwidth, per cluster by $\frac{D}{R}=\sqrt{3 N_{c}}[18]$. 
the basis of a degree of partial interleaving which corresponds to the maximum tolerable delay (typically $60 \mathrm{~ms}$ for speech applications) and complexity (storage costs). One could try to use a purely analytical approach as in [166] where performance bounds were derived to determine the degradation resulting from partial interleaving with respect to the ideal interleaving case. However, although [166] takes into account diversity, it is strictly concerned with flat fading and AWGN. It remains to be seen if adding cochannel interference would require some sort of gaussian assumption to make the analysis tractable. If so, some sort of semi-analytical process with the method of moments may be preferable to obtain reliable results. These could then also be translated into cochannel reuse ratio improvements as suggested in Section 6.2.1.

\subsubsection{More powerful codes and simplified decoding algorithms}

The results of this thesis are based on a convolutional code with a short constraint length of 3 and soft-decision Viterbi decoding. Coded system performance could be improved with longer constraint lengths but only at the expense of increased decoder complexity. As the latter is of prime concern for the portable applicati in, it would thus be worthwhile to consider using these more powerful codes in conjunction with reduced complexity decoding algorithms such as the $\mathbf{M}$-algorithm [215] or different sequential decoding algorithms e.g. the Fano algorithm [216] or the stack algorithm $[217,218]$. However, such sequential decoding algorithms typically yield a larger decoding delay which may limit their use to data (as opposed to voice) applications.

\subsubsection{Trellis coding}

Trellis codes avoid bandwidth expansion by introducing redundancy in an expanded constellation set. The performance of trellis codes in conjunction with equalization on a frequency-selective indoor channel corrupted by AWGN has recently been studied [151] via simulation for the case of no interleaving. However, as discussed in Chapter 4, execution time constraints generally preclude the generation of statistically significant bit-by-bit simulation results on such a slowly varying channel. It may therefore be of interest to use analytical or semi-analytical approaches and include the effects of interference as well as to study the case of partial interleaving corresponding to a given 
complexity and delay constraint. As pointed out in Chapter 2 and as was done in [151], relatively small constellation sets are preferable (e.g. 8PSK) as large sets do not perform well on highly dispersive channels.

\subsubsection{Evaluations based on refined channel models}

The cluster channel model developed in [60] was based on measurements in one medium-size office building. Performance evaluations would be more significant if these were based on averages over different channel models representing different portable environments such as offices, manufacturing floors, warehouses, campuses, hospitals, shopping malls etc. These evaluations should also be based on refined channel models stch as a cluster model with a modified Poisson path arrival process [90].

\subsubsection{Capacity of indoor radio links}

The full potential of wideband TDMA portable indoor radio links can be evaluated by determining information theoretic capacity bounds for these channels. The general problem of calculating the capacity of a multi-input, multi-output dispersive link with additive white Gaussian noise has been considered by Brandenburg and Wyner [219] and later by Amitay and Salz [183] for the dually polarized digital microwave radio channel. As discussed in Chapter 2, Winters [181] then applied these techniques to calculate the average ${ }^{83}$ total capacity or average capacity per portable for the limiting case of independent flat Rayleigh fading channels between $(\mathrm{N}+1)$ portables (each with one antenna) and a base station with $M$ antennas. It is thus of interest to obtain similar bounds based on the more realistic cluster model [60] employed in Chapter 5. This will provide information as to whether the large number of resolvable uncorrelated multipath echoes at high signaling rates (i.e. frequency-selective case) can improve capacity with respect to the single ray model (flat fading case) considered in [181]. For this purpose, a procedure based on the analytical techniques of Amitay and Salz [183] is described in appendices $D$ and $E$.

83 As the channel is time-varying, the capacity is a random variable. 


\section{Bibliography}

[1] D. Cox, "Universal digital portable communications," Proceedings of the IEEE, vol. 75 , no. 4, pp. 436-477. April 1987.

[2] D. Cox, H. Amold, and P. Porter, "Universal digital portable communications: a system perspective." IEEE Journal on Selected Areas in Communications, vol. 5. nn. SAC-5, pp. 764-773, June 1987.

[3] H. Shosteck, "Price and demand - The factors affecting growth of the cellular market," in Proceedings of the fourth Nordic Seminar on digital mobile radio communications. (Oslo, Norway), pp. 1.5.1-1.5.7, June 1990.

[4] H. Shosteck, "The future U.S. ntarket for portable cellular telephones," in Proceedings of the third Nordic Seminar on digital mobile radio communications. (Copentagen. Denmark), pp. 14.1.1-14.1.8, September 1988.

[5] D. McFarlane, "European personal communications using microcells," in Proceedings of the IEE Colloqium on Microcellular Mobile Radio, (London, U.K.), February 1989.

[6] P. Giusto, "Towards personal communications," CSELT Technical Reports, vol. 19, no. 1. pp. 1-6, February 1991.

[7] S. Ginn, "Personal communication services: expanding the freedom to communicate." IEEE Communications Magazine, vol. 29, no. 2, pp. 30-39, February 1991.

[8] R. Singer and D. Invin, "Personal communication services: the next technological revolution," IEEF. Communication: Marazine, vol. 29, no. 2, pp. 62-66. February 1991.

[9] T. Hattori, H. Sekiguchi, K. Kohiyama, and H. Yamamofo, "Personal Conmunication concept and architecture," in Proceedings of the 1990 IEEE International Conference on Communications, (Atlanta, Ga.), pp. 1351-1357, April 1990.

[10] D. Cox, "Personal communicitions: a viewpoint," IEEE Communications Magazine, vol. 28 , no. 11 , pp. 10-20, November 1990.

[11] R. Steele, "Deploying personal communication networks," IEEE Communications Magazine, vol. 28, no. 9, pp. 12-15, September 1990.

[12] T. Bel' Glnt. c:llular system planned," The Institute, vol. 22, no. 3, p. 3, September 199.

[13] O. Eriksson :," 5 . Bjömdal, "Personal telecommunications: a possible evolution in - near fuluv , f tc tay's digital mobile telecommunications systems and intelligent zetworks" in :rocesdings of the fourth Nordic Seminar on digital mobile radio communications, iOslo, Norway), pp. 8.3.1-8.3.7, June 1990.

[14] R. Gibson, "Towards a universal mobile communications system," in Proceedings of the first Rutgers worishop on third generation wireless information networks. (East Brunswick, N.J.), pp. 145-150, June 1989. 
[15] K. Walter, "Personal telecommunications: a new dimension in teleservice accessibility," in Proceedings of the fourth Nordic Seminar on digita! mobile radio communications, (Oslo, Norway), pp. 8.4.1-8.4.7, June 1990.

[16] K. Kohiyama, T. Hattori, H. Sekiguchi, and R. Kawasaki, "Advanced personal communication system," in Proceedings of the 1990 IEEE Vehicular Technology Conference, (Orlando, Fl.), pp. 161-166, May 1990.

[17] R. Steele, "Towards a high-capacity digital cellular mobile radio system," Proceedings of the IEE (part F), vol. 132, no. 5, pp. 405-415, August 1985.

[18] V. MacDonald. "The cellular concept," Bell System Technical Journal, vol. 58, no. 1, F. 15-41, January 1979.

[19' W. Young, "AMPS: Introduction, background and objectives," Bell System Technical Journal, vol. 58, no. 1, pp. 1-14, January 1979.

[20] J. Rémy, J. Cueugniet, and C. Siben, Systemes de radiocommunications avec les mobiles. Paris: Eyrolles, 1988.

(21] J. Walker, Mobile information systems. Boston, Ma.: Artech House, 1990.

[22] D. Cox, "Portable digital radio communications: an approach to tetherless access," IEEE Communications Magazine, vol. 27, no. 5, pp. 30-40, July 1989.

[23] R. Stecle. "The cellular environment of lightweight handheld portables," IEEE Communications Magazine, vol. 27, no. 7, pp. 20-29, July 1989.

[24] D. Akerberg, "Propertics of a TDMA picocellular office communication system," in Proceedings of the 1989 IEEE Vehicular Technology Conference, (San Francisco, Ca.), pp. 186-191, May 1989.

[25] R. Muammar and S. Gupta, "Cochannel interference in high-capacity mobile radio systems," IEEE Transactions on Communications, vol. COM-30, no. 8, pp. 1973-1978, August 1982.

[26] D. Goodman, "Cellular packet communications," IEEE Transactions on Communications, vol. COM-38, no. 8, pp. 1272-1280, August 1990.

[27] D. Goodman, "Second generation wireless information networks," IEEE Transactions on Vehicular Technoloyy, vol. 40, no. 4, pp. 366-374 May 1991.

[28] D. Goodman, "Trends in cellular and cordless communications," IEEE Comunications Magazine, vol. 29, no. 6, pp. 31-41, June 1991.

[29] B. Mallinder, "An overview of the GSM system," in Proceedings of the third Nordic Seminar on digital mobile radio communications, (Copenhagen, Denmark), pp. 3.1.13.1.13, September 1988.

[30] M. Alvernhe and D. Bames, "GSM services and facilities fo the user," in Proceedings of the fourth Nordic Seminar on digital mobile radio comn ;nications, (Oslo, Norway), pp. 15.1.1-15.1.12, June 1990. 
[31] A. Maloberti, "Radio transmission interface of the digital pancuropean mobile system." in Proceedings of the 1989 IEEE Vehicular Technology Conference, (San Francisco, Ca.). pp. 712-717, May 1989.

[32] A. Maloberti, "Some aspects of the GSM radio interface." in Proceedings of the third Nordic Seminar on digital mobile radio communications, (Copenhagen, Dermark), pp. 4.1.1-4.1.11, September 1988.

[33] B. Ghillebaert, "Le système cellulaire de télécommunication avec les mobiles," Annales des Télécommunications, vol. 42, no. 7, pp. 432-438, July 1987.

[34] H. Ochsner, "DECT - Digital European Cordless Telecommunications," in Proceedings of the 1989 IEEE Vehicular Technology Conference, (San Francisco, Ca.), pp. 718-721. May 1989.

[35] M. Heath, "Propagation measurements at $1.76 \mathrm{GHz}$ for Digital European Condless Telecommunications," in Proceedings of the 1990 Global Telecommunications Confer. ence, (San Diego, Ca.), pp. 1007-1012, December 1990.

[36] L. Lopes, "On the radio link performance of the Digital European Cordless Telecommunications system," in Proceedings of the 1990 Global Telecommunications Conference, (San Diego, Ca.), pp. 1013-1017, December 1990.

[37] R. Potter, "Personal communication networks," in Proceedings of the fourth Nordic Seminar on digital mobile radio communications, (Oslo, Norway), pp. 8.2.1-8.2.5. June 1990.

[38] D. Grillo and G. MacNamee, "European perspectives on third generation personal communication systems," in Proceedings of the 1990 IEEE Vehicular Technology Conference, (Orlando, Fl.), pp. 135-140, May 1990.

[39] K. Raith and J. Uddenfelt, "Capacity of digital cellular TDMA systems," IEEE Transactions on Vehicular Technology, vol. 40, no. 4, pp. 323-332, May 1991.

[40] K. Kinoshita, M. Hata, and K. Hirade, "Digital mobile telephone system using a TD/FDMA scheme," IEEE Transactions on Vehicular Technology, vol. VT-31, no. 4. pp. 153-160, November 1982.

[41] A. Saleh, A. Rustako, and R. Roman, "Distributed antennas for indoor radio communications," IEEE Transactions on Communications, vol. 35, no. 12, pp. 1245-1251, Dec. 1987.

[42] J. Chuang. "The effect of time delay spread on portable radio communication channels with digital modulation," IEEE Journal on Selected Areas in Communications, vol. SAC5, no. 6, pp. 879-889, June 1987.

[43] M. Kavehrad and G. Bodeep, "Design and experimental results for a direct-sequence spread spectrum radio using differential phase-shift keying modulation for indoor, wireless communications," IEEE Journal on Selected Areas in Communications. vol. SAC-5, no. 5, pp. 815-823, June 1987. 
[44] W. Lee, "Overview of cellular CDMA," IEEE Transactions on Vehicular Technology, vol. 40, no. 2, pp. 291-302, May 1991.

[45] K. Gilhousen, I. Jacobs, R. Padovani, A. Viterbi, L. Weaver, and C. Wheatley, "On the capacity of a cellular CDMA system," IEEE Transactions on Vehicular Technology, vol. 40, no. 4, pp. 303-312. May 1991.

[46] J. Holtzman, "Power control in CDMA for digital cellular networks," in Proceedings of the second Rutgers workshop on third generation wireless information networks, (East Brunswick, N.J.), pp. 264-273, October 1990.

[47] J. Taylor and J. Omura, "Spread-spectrum technology: a solution to the personal cornmunication services frequency allocation dilemma," IEEE Communications Magazine, vol. 29, no. 2, pp. 48-51, February 1991.

[48] D. Schilling, R. Pickholtz, and L. Milstein, "Spread-spectrum goes commercial," IEEE Communications Magazine, vol. 28, no. 8, pp. 40-45, August 1990.

[49] P. Monsen, "MMSE equalization of interference on fading diversity channels," IEEE Transactions on Communications, vol. COM-34, no. 1, pp. 5-12, January 1984.

[50] P. Monsen, "Theoretical and measured performance of a DFE modem on a fading multipath channel," IEEE Transactions on Communications, vol. COM-25, no. 10, pp. 1144-1153, October 1977.

[51] C. Baird and C. Zahm, "Performance criteria for narrow-band array processing," Journat of the American Institute of Decision Sciences, pp. 564-565, December 1971.

[52] J. Winters, "Optimum combining for indoor radio systems with multiple users," IEEE Transactions on Communications, vol. COM-35, no. 11, pp. 1222-1230, November 1987.

[53] J. Winters, "Optimum combining in digital mobile radio with cochannel interference," IEEE Journal on Selected Areas in Communications, vol. SAC-2, no. 4, pp. 528-539, July 1984.

[54] J. Rasinger, A. Scholtz, W. Pickler, and E. Bonek, "A new enhanced-bandwidth intemal antenna for portable communication systems," in Proceedings of the 1990 IEEE Vehicular Technology Conference, (Orlando, F1.), pp. 7-12, May 1990.

[55] K. Tsunekawa, "Diversity antennas for portable telephones," in Proceedings of the IEEE 1989 Vehicular Technology Conference, (San Francisco, Ca.), pp. ¿0-56, May 1989.

[56] Y. Miyagaki, N. Morinaga, and T. Namekawa, "Effect of compound strategies of error correcting coding, bit interleaving and diversity combining in mobile radio data transmission." Transactions of the Institute of Electronics and Communications Engineers of Japan, vol. J67-B, no. 6, pp. 599-606, June 1984.

[57] A. Viterbi and J. Omura, Principles of Digital Communications and Coding. New York: McGraw-Hill, 1979. 
[58] S. Mui and J. Modestino, "Comparison of convolutional and block codes on the Rician channel," in Proceedings of the 1977 IEEE National Telecommunications Conference. (Los Angeles, Ca.), pp. 20.4.1-20.4.7, December 1977.

[59] C. Helstrom, "Calculating error probabilities for intersymbol and cochannel interference," IEEE Transactions on Communications, vol. COM-34, no. 5, pp. 430-435, May 1986.

[60] A. Saleh and R. Valenzuela, "A statistical model for indoor multipath propagation," IEEE Journal on Selected Areas in Communications, vol. SAC-5, no. 2, pp. 128-137, February 1987.

[61] C. Despins, D. Falconer, and S. Mahmoud, "Coding and optimum baseband combining for wide-band TDMA indoor wireless channels," Canadian Journal of Electrical and Computer Engineering, vol. 16, no. 2, pp. 53-62, April 1991.

[62] C. Despins, D. Falconer, and S. Mahmoud, "Coding and optimum baseband combining for wide-band TDMA indoor wireless channels," in Proceedings of the 1990 IEEE Global Telecommunications Conference, (San Diego, Ca.), pp. 1832-1837. December 1990.

[63] C. Despins, D. Falconer, and S. Mahmoud, "Compound strategies of coding, equalization. and space diversity for wide-band TDMA indoor wireless channels." in Proceedings of the IEEE International Symposium on Personal, Indoor and Mobile Radio Communications. (London, U.K.), September 1991.

[64] C. Despins, D. Falconer, and S. Mahmoud, "Compound strategies of coding, equalization. and space diversity for wide-band TDMA indoor wireless channels," submitted to IEEE Transactions on Vehicula. Technology.

[65] S. Stein, "Fading channel 'ssues in system engineering," IEEE Journal on Selected Areas in Communications, vol. SAC-5, no. 2, pp. 68-89, February 1987.

[66] P. Bello, "Characterization of randomly time-variant linear channels," IEEE Transactions on Communications, vol. COM-11, no. 12, pp. 360-393. December 1963.

[67] M. Sylvain, "Panorama des études sur les trajets multiples," Annales des Télécommunications, vol. 40, no. 11, pp. 547-564, November 1985.

[68] D. Cox, "910 MHz urban mobile radio propagation: multipath characteristics in New York City." IEEE Transactions on Communications, vol. COM-21, no. 11, pp. 11881194, November 1973.

[69] A. Papoulis, Probability, Random Variables and Stochastic Processes. New York: McGraw-Hill, 1984.

[70] L. Rayleigh, "On the resultant of a large number of vibrations of the same pitch and of arbitrary phase," Philosph. Magazine and J. Sci., vol. 10, no. 60, p. 73, August 1880.

[71] J. Proakis, Digital Communications. New York: McGraw-Hill, 1983.

[72] W. Jakes, Jr., Microwave Mobile Communications. New York: John Wiley and Sons, 1974. 
173] R. French, "The effect of fading and shadowing on channel reuse in mobile radio," IEEE Transactions on Vehicular Technology, vol. VT-28, no. 4, pp. 171-181, August 1979.

174] S. Rice, "Statistical properties of a sine wave plus random noise," Bell System Technical Journal, vol. 27, no. 1, January 1948.

[75] R. Bultitude. "Measurement, characterization and modeling of indoor $800 / 900 \mathrm{Mhz}$ radio channels for digital communications," IEEE Communications Magazine, vol. 25, no. 6, pp. 5-12, June 1987.

[76] M. Nakagami, Statistical methods in radio wave propagation. New York: Pergamon Press, 1960.

[77] U. Charash, "Reception through Nakagami fading multipath channels with random delays," IEEE Transactions on Communications, vol. COM-27, no. 4, April 1979.

[78] N. Shepherd, "Radio wave loss deviation and shadow loss at 900 Mhz," IEEE Transactions on Vehicular Technology, vol. VT-26, no. 4, November 1977.

[79] B. Glance and L. Greenstein, "Frequency-selective fading effects in digital mobile radio wit), diversity combining." IEEE Transactions on Communications, vol. COM-31, no. 9, pp. 1085-1094, September 1983.

[80] A. Saleh and L. Cimini, "Indoor radio communications using time-division multiple access with cyclical slow frequency hopping and coding," IEEE Journal on Selected Areas in Communications, vol. SAC-7, no. 1, pp. 59-70, January 1989.

[81] D. Devasirvatham, "The delay spread measurements of wide-band radio signals within a building," Electronics Letters, vol. 20, no. 11, pp. 950-951, November 1984.

[82] S. Alexander, "Radio propagation within buildings at $900 \mathrm{MHz}$," Electronics Letters, vol. 18, no. 21, pp. 913-914, October 1982.

[83] S. Alexander, "Characterizing buildings for propagation at $900 \mathrm{MHz}$," Electronics Letters, vol. 19, no. 20, p. 860, September 1983.

[84] H. Hoffman and D. Cox, "Attenuation of $900 \mathrm{MHz}$ radio waves propagating into a metal building," IEEE Transactions on Antennas and Propagation, vol. AP-30, no. 7 , pp. 808-811. July 1982.

[85] G. Ott and A. Plitkins, "Urban path-loss characteristics at $820 \mathrm{MHz}$," IEEE Transactions on Vehicular Technology, vol. VT-27, no. S, pp. 189-197, November 1978.

[86] P. Harley. "Short distance attenuation measurements at $900 \mathrm{MHz}$ and $1.8 \mathrm{GHz}$ using low antenna heights for microcells," IEEE Journal on Selected Areas in Communications, vol. SAC-7, no. 1, pp. 5-11, January 1989.

[87] D. Devasirvatham. "A comparison of time delay spread and signal level measurements within two dissimilar office buildings," IEEE Transactions on antennas and propagation, vol. 20, no. 3, pp. 319-324, March 1987. 
[88] S. Alexander and G. Pulgliese, "Cordless communication within buildings: results of measurements at $900 \mathrm{MHz}$ and at $60 \mathrm{GHz}$, " British Telecom Technical Journal. pp. 99 105, 1983.

[89] C. Gutzeit, " $900 \mathrm{Mhz}$ indoor/outdoor propagation investigations via bit crror structure measurements," in Proceedings of the 1989 IEEE Vehicular Technology Conference, (San Francisco, Ca.), pp. 321-325, May 1989.

[90] R. Ganesh and K. Pahlavan. "On the modeling of fading multipath indoor radio channels," in Proceedings of the 1989 IEEE Global Telecommunications Conference. (Dallas, Tx.), pp. 1346-1350, November 1989.

[91] R. Ganesh and K. Pahlavan, "On the arrival of paths in fading multipath indoor radio channels," Electronics Letters, vol. 25, no. 12, pp. 763-765, March 1989.

[92] H. Suzuki, "A statistical model for urban radio propagation," IEEE Transactions on Communications, vol. COM-2, no. 7, pp. 673-680, July 1977.

[93] G. Turin and F. Clapp. "A statistical model of urban multipath propagation." IEEE Transactions on Vehicular Technology, vol. VT-21, no. 1, pp. 1-9. February 1972.

[94] T. Sexton and K. Pahlavan, "Channel modeling and adaptive equalization of indoor radio channels," IEEE Journal on Selected Areas in Communications. vol. SAC-7, no. 1 , pp. 114-120. January 1989.

[95] K. Pahlavan and R. Ganesh, "Time and frequency domain models for indoor radio propagation," in Proceedings of the IEEE workshop on wireless LAN's, (Worcester. Ma.), pp. 73-80, May 1991.

[96] S. Howard and K. Pahlavan, "Statistical autoregressive models for the indoor radio channel," in Proceedings of the 1990 IEEE Global Telecommunications Conference, (San Diego, Ca.), pp. 1341-1345, December 1990.

[97] J. Parsons, A. Turkmani, and F. Ju. "The effect of base station antenna height on 900 Mhe microcellular mobile radio systems," in Proceedings of the IEE Colloqium on Microcellular Mobile Radio, (London, U.K.), February 1989.

[98] B. Gudmundson and O. Grimlund, "Handoff in microcellular based personal tclephone systems," in Proceedings of the second Rutgers workshop on third generation wireless information networks, (East Brunswick, N.J.), pp. 160-171, October 1990.

[99] P. Bello and B. Nellin. "The effect of frequency selective fading on the binary error probability of incoherent and differentially coherent matched filter receivers," IEEE Transactions on Communications, vol. COM-11, no. 6, pp. 170-186, June 1963.

[100] C. Bailey and J. Lindenlaub, "Further results conceming the effect of frequency selective fading on differentially coherent matched filter receivers," IEEE Transactions on Communications, vol. COM-11, no. 10, pp. 749-751, October 1968. 
[101] D. Cox and R. Leck, "Distributions of multipath delay spread and average excess delay for $910 \mathrm{MHz}$ urban mobile radio path," IEEE Transactions on Antennas and Propagation. vol. AP-23, no. 3, pp. 206-213, March 1975.

[102] J. Winters and Y. Yeh, "On the performance of wide-band digital radio transmission within buildings using diversity," in Proceedings of the 1985 IEEE Global Telecommunications Conference, (New Orleans, Lo.), pp. 991-996, November 1985.

[103] H. Whecler, "Small antennas," IEEE Transactions on Antennas and Propagation, vol. AP23. no. 7. pp. 462-469, July 1975.

[104] T. Taga, K. Tsunoda, and H. Imahori, "Correlation properties of antenna diversity in indoor mobile communication environments," in Proceedings of the IEEE 1989 Vehicular Technology Conference, (San Francisco, Ca.), pp. 446-451, May 1989.

[105] R. Bultitude, S. Mahmoud, and W. Sullivan, "A Comparison of indoor radio propagation characteristics at $910 \mathrm{MHz}$ and $1.75 \mathrm{GHz}$," IEEE Journal on Selected Areas in Communications, vol. SAC-7, no. 1. pp. 20-30, January 1989.

[106] D. Devasirvatham, C. Banerjec, M. Krain, and D. Rappaport, "Multi-frequency radiowave propagation measurements in the portable radio environment," in Proceedings of the 1990 IEEE International Conference on Communications, (Atlanta, Ga.), pp. 1334-1340, April 1990.

[107] R. Bemhardt, "User access in portable radio systems in a cochannel interference environment," IEEE Journal on Selected Areas in Communications, vol. SAC-7, no. 1, pp. 49-58. January 1989.

[108] D. Cox, "Cochannel interference considerations in frequency-reuse small coverage-area radio systems," IEEE Transactions on Communications, vol. COM-30, no. 1, pp. 135142. January 1982.

[109] T. Brenig, "Interference control in ceilular systems," in Proceedings of the 1985 IEEE Vehicular Technology Conference, (Boulder, Co.), pp. 32-37, May 1985.

[110] 3. Chuang, "A high performance diversity selection technique for TDMA portable radio communications," in Proceedings of the 1989 IEEE Global Telecommunications Conference, (Dallas, Tx.), pp. 1361-1365, November 1989.

[111] R. Bemhardt, "Macroscopic diversity in frequency-reuse radio systems," IEEE Journal on Selected Areas in Communications, vol. SAC-5, no. 5, pp. 862-870, June 1987.

[112] L. Chang and J. Chuang, "Diversity selection using coding in a portable radio communications channel with frequency-selective fading." IEEE Journal on Selected Areas in Communicaions, vol. SAC-7, no. 1, pp. 89-98, January 1989.

[113] H. Amold, R. Murray, and D. Cox, "Macroscopic diversity in the portable radiocomunnication environment," in Proceedings of the 1985 North American Radio Science meeting. (Vancouver, Canada), June 1985. 
[114] J. V. Rees, "Cochannel measurements for interference limited small-cell planning," Archiv fur Elektronik und Ubertragungstechnik, vol. 41, no. 5, pp. 318-320, September 1987.

[115] Y. Yeh and S. Schwartz, "Outage probability in mobile telephony due to multiple log-normal interferers," IEEE Transactions on Communications, vol. COM-32, no. 4. pp. 380-388, April 1984.

[116] L. Fenton. "The sum of log-normal probability distributions in scatter transmission systems," IRE Transactions on Comminication Systems, vol. CS-9, no. 3, pp. 57-67. March 1960.

[117] R. Bemhardt, "The character of cochannel interference in frequency-reuse radio systems," Private communication, March 1989.

[118] N. Kinoshita and S. Sampei, "Method of rejecting adjacent channel interference using an adaptive equalizer," Transactions of the Institute of Electronics and Communication Engineers of Japan, vol. J71-B, no. 10, pp. 1119-1126, October 1988.

[119] W. Yung. "Outage and error probabilities of QPSK with diversity reception in Rayleigh fading and Log-normal shadowing channel," in Proceedings of the IEEE 1989 International Conference on Communications, (Boston, MA.), pp. 182-189, Junc 1989.

[120] R. Bernhardt and P. Porter. "Cost comparisons for macroscopic diversity architectures in universal digital portable communications," in Proceedings of the 1985 IEEE Global Telecommunications Conference, (New Orieans, Lo.), pp. 1008-1012. December 1985.

[121] A. Acampora and J. Winters, "System applications for wireless indoor communications," IEEE Communinations Magazine, vol. 25, no. 8, pp. 11-20, August 1987.

[122] A. Saleh, A. Rustako, L. Cimini, G. Owens, and R. Roman, “An experimental TDMA indoor radio communications system using slow frequency hopping and coding," IEEE Transactions on Communications, vol. 39, no. 1, pp. 152-162, January 1991.

[123] D. Cox, "Antenna diversity performance in mitigating the effects of ponable radiotelephone orientation and multipath propagation," IEEE Transactions on Communications, vol. COM-31, no. 5, pp. 620-628, May 1983.

[124] R. Valenzuela, "Perfomance of quadrature amplitude modulation for indoor radio communications," IEEE Transactions on Communications, vol. COM-35, no. I1, pp. 1236-1238, November 1987.

[125] J. Proakis, "Adaptive equalization for TDMA digital mobile radio," IEEE Transactions on Vehicular Technology. vol. 40, no. 4, pp. 333-341. May 1991.

[126] J. Chuang, "The effects of multipath delay spread on timing recovery," IEEE Transactions on Vehicular Technology, vol. VT-35, no. 3, pp. 135-140, August 1987.

[127] P. Monsen, "Feedback equalization for fading dispersive channels," IEEE Transactions on Information Theory, vol. IT-16, no. 1, pp. 56-64, January 1971. 
[128] P. Monsen, "Digital transmission performance on fading dispersive diversity channels," IEEE Transactions on Communications, vol. COM-21, no. 1, pp. 33-39, January 1973.

[129] P. Monsen, "Adaptive equalization of the slow fading channel," IEEE Transactions on Communications, vol. COM-22, no. 8, pp. 1064-1075, August 1974.

[130] H. Sari, "Baseband equalizer performance in the presence of selective fading," in Proceedings of the 1983 IEEE GLbal Telecommunications Conference, December 1983.

[131] A. Leclert and P. Vandamme, "Decision feedback equalization of dispersive radio channels," IEEE Transactions on Communications, vol. COM-33, no. 7, pp. 676-684, July 1985.

[132] J. Mazo, "Optimum timing phase for an infinite equalizer," Bell System Technical Journal, vol. 54, no. 1, pp. 189-200, January 1975.

[133] R. Gitlin and S. Weinstein, "Fractionally-spaced equalization: an improved transversal digital filter," Bell System Technical Journal, vol. 60, no. 2, February 1981.

[134] G. Ungerboeck, "Fractional tap-spacing equalizer and consequences for clock recovery in data modems," IEEE Transactions on communications, vol. COM-24, no. 8, pp. 856864. August 1976.

[135] P. Vandamme, M. Joindot, and $O$. Acx, "Performances asymptotiques des égaliseurs fractionnes," Annales des Télecommunications, vol. 42, no. 10, pp. 517-526, October 1987.

[136] N. Amitay and L. Greenstein, "Multipath outage performance of digital radio receivers using fitsite-tap adaptive equalizers," IEEE Transactions on Communiucations, vol. COM32. no. \&. pp. 597-608, May 1984.

[137] R. Agusti, F. Casadevall, and J. Olmos, "Performance of fractioned and non-fractioned equalizers with high-level QAM," IEEE Journal on Selected Areas in Communications, vol. SAC-5, no. 3, pp. 476-483, April 1987.

[138] P. Niger and P. Vandamme, "Étude comparative des techniques d'égalisation synchrone et fractionnée pour transmissions numériques par voie hertzienne," Annales des Telécommunications, vol. 44, no. 8, pp. 363-373, August 1989.

[139] G. Fomey, Jr., "Maximum-likelihood sequence estimation of digital sequences in the presence of intersymbol interference," IEEE Transactions on Information Theory, vol. IT18. no. 5, pp. 363-378, May 1972.

[140] J. Salz, "On mean-square decision-feedback equalization and timing phase," IEEE Transactions on communications, vol. COM-25, no. 12, pp. 1471-1476, December 1977.

[141] K. Brunner and C. Weaver, "A comparison of synchronous and fractional-spaced DFE's in a multipath fading environment," in Proceedings of the 1988 IEEE Global Telecommunications Conference, (Hollywood, F1.), pp. 1452-1456, November 1988. 
[142] H. Suzuki, T. Ueda, and A. Higashi, "High bit-rate digital mobile radio transmission with a decision feedback equalizer," in Proceedings of the 1989 IEEE International Conference on Communications, (Boston, Ma.), pp. 171-175. June 1989.

[143] S. Qureshi, "Adaptive equalization," Proceedings of the IEEE, vol. 73, no. 9, pp. 13491387, September 1985.

[144] M. Clark, W. Kennedy, M. Shafi, and L. Greenstein, "MMSE diversity combining for wide-band digital cellular radio," in Proceedings of the 1990 Global Telecommunications Conference, (San Diego, Ca.), pp. 495-499, December 1990.

[145] R. Valenzuela, "Performance of adaptive equalization for indoor radio communications," IEEE Transactions on Communications, vol. COM-37, no. 3, pp. 291-293, March 1989.

[146] W. Wong and L. Greenstein. "Multipath fading models and adaptive equalizers in microwave digital radio," IEEE Transactions on Communications, vol. COM-32, no. 8, pp. 928-934, August 1984.

[147] S. Howard and K. Pahlavan. "Performance of a DFE modem evaluated from measured indoor radio multipath profiles," in Proceedings of the 1990 IEEE International Conference on Communications, (Atlanta, Ga.), pp. 1341-1345, April 1990.

[148] K. Pahlavan, R. Ganesh, and T. Hotaling, "Multipath propagation measurements on manufacturing floors at $910 \mathrm{Mhz}$," Electronics Letters, vol. 3, pp. 225-227, February 1989.

[149] D. Divsalar and M. Simon, "Trellis coded modulation for $4800-9600$ bits/s transmission over a fading mobile satellite channel," IEEE Journal on Selected Areas in Communications, vol. SAC-5, no. 2, pp. 162-175, February 1987.

[150] S. Hanna, M. El-Tanany, and S. Mahmoud, "Simulated performance of coded digital transmission in a multi-user indoor communications environment," in Proceedings of the 1991 IEEE Vehicular Technology Conference, (St.Louis, Mo.), pp. 234-239, May 1991.

[151] R. Paiement and J. Chouinard, "Simulated application of trellis coded modulation to the indoor wireless channel," in Proceedings of the I991 IEEE Vehicular Technology Conference, (St.Louis, Mo.), pp. 216-221, May 1991.

[152] A. Levy, "Performances en probabilités d'erreur des modulations à grand nombre d"śtats en période d'évanouissements sélectifs," Annales des télécommunications, vol. 40, no. 12. pp. 617-625, December 1985.

[153] P. Mabey, "Mobile radio data transmission: coding for error control," IEEE Transactions on Vehicular Technology, vol. VT-27, no. 3, pp. 99-109, August 1978.

[154] S. Lin and D. Costello, Error Control Coding: Fundamentals and Applications. Englewood Cliffs (New Jersey): Prentice-Hall, 1983.

[155] K. Mokrani and S. Soliman, "Reed-Solomon codes over fading dispersive channels," in Proceedings of the 1988 IEEE Vehicular Technology Conference, (Philadelphia, Pa.). pp. 497-502, May 1988. 
[156] S. Soliman and K. Mokrani, "Performance of coded systems over fading dispersive channels," submitted to IEEE Transactions on Communications.

[157] D. Chase, "A class of algorithms for decoding block codes with channel measuremeni information," IEEE Transactions on Information Theory, vol. IT-18, no. 1, pp. 170-182, January 1972.

[158] G. Forney, Jr., "The Viterbi algorithm," Proceedings of the IEEE, vol. 61, no. 3, pp. 268278, March 1973.

[159] A. Viterbi, "Convolutional codes and their performance in communication systems," IEEE Transactions on Communications, vol. COM-19, no. 5, pp. 751-772, October 1971.

[160] J. Hagenauer, "Viterbi decoding of convolutional codes for fading and burst channels," in Proceedings of the 1980 Zurich Seminar on Digital Communications, (Zurich, Switzerland), pp. G2.1-G2.7, August 1980.

[161] J. Ramsey, "Realization of optimum interleavers," IEEE Transactions on information theory, vol. IT-16, no. 3, pp. 338-345, May 1970.

[162] G. Forney, Jr., "Burst-correcting codes for the classic bursty channel," IEEE Transactions on Communications, vol. COM-19, no. 10, pp. 772-781, October 1971.

[163] R. Kris: namurthi and S. Gupta, "Coding for portable radios," in Proceedings of the IEEE 1989 Vehicular Technology Conference, (San Francisco, Ca.), pp. 660-665, May 1989.

[164] L. Chang and S. Ariyavisitakul, "Performance of convolutional codes with interleaving in the interference limited Rayleigh fading channel," in Proceedings of the IEEE 1991 Vehicular Technology Conference, (St.Louis, Mo.), pp. 812-816, May 1991.

[165] F. Gagnon, D. Haccoun, and C. Leung, "An analysis of convolutional coding for land mobile radio channels," in Proceedings of the IEEE 1989 Vehicular Technology Conference, (San Francisco, Ca.), pp. 660-670, May 1989.

[166] D. Haccoun and F. Gagnon, "Performance analysis of error control coding for mobile channels," in Proceedings of the 1990 Global Telecommunications Conference, (San Diego, Ca.), pp. 1805-1809, December 1990.

[167] E. Lutz, "Code and interleaver design for data transmission over fading channels," in Proceedings of the 1984 IEEE Global Telecommunications Conference, (Atlanta, Ga.), pp. 381-386, November 1984.

[168] G. Battail and A. WI-Sherbini, "Coding for radio channels," Annales des Télécommunications, vol. 37, no. 1-2, pp. 75-96, January 1982.

[169] C. Cleverly and P. Waters, "Transmission of ISDN signals over mobile radio channels using BPSK, decision feedback equalisation and Reed Solomon coding," in Proceedings of the IEE Collogium on methods of combating multipath effects in wide-band digital cellular mobile systems, (London, U.K.), Ociober 1987. 
[170] M. Uesugi, K. Honma, and K. Tsubaki, "Adaptive equalization in TDMA digital mobile radio," in Proceedings of the 1989 IEEE Global Telecommunications Conference, (Dallas, TX.), pp. 95-101, November 1989.

[171] B. Widrow, P. Mantey, and L. Griffiths, "Adaptive antenna systems," Proceedings of the IEEE, vol. 55, no. 12, pp. 2143-2163, December 1967.

[172] V. Bogachev and 1. Kiselev, "Optimum combining of signals in space diversity reception," Telecommunications and Radio Engineering, vol. 34-35, pp. 83-85, October 1980.

[173] R. Vaughan, "On optimum combining at the mobile," IEEE Transactions on Vehicular Technology, vol. VT-37, no. 4, pp. 181-188, November 1988.

[174] P. Henry and B. Glance. "A new approach to high capacity digital mobile radio." Bell System Technical Journal, vol. 60, no. 10, pp. 1891-1904, Octoher 1981.

[175] S. Hanna, M. El-Tanany, and S. Mahmoud, "An adaptive combiner for cochannel interference reduction in multi-user indoor radio systems," in Proceedings of the 199I IEEE Vehicular Technology Conference, (St.Louis, Mo.), pp. 222-227, May 1991.

[176] C. Pauw, "Application of decision feedback equalisers in an environment with narrowband interfering signals," Electronics Letters, vol. 16, no. 20, pp. 787-788, Scptember 1980.

[177] L. Li and L. Milstein, "Rejection of CW interference in QPSK systems using decision feedback filters," IEEE Transactions on Communications, vol. COM-31, no. 4, pp. 473483. April 1983.

[178] W. V. Etten, "Maximum likelihood receiver for multiple channel transmission systems," IEEE Transactions on Communications, vol. 24, no. 2, pp. 276-283, February 1976.

[179] C. Shannon and W. Weaver, The Mathematical Theory of Communication. Urbana, Illinois: University of Illinois Press, 1949.

[180] W. Lee, "Estimate of channel capacity in Rayleigh fading environment," in Proceedings of the 1988 IEEE Vehicular Technology Conference, (Philadclphia, Pa.). pp. 582-584, May 1988.

[181] J. Winters, "On the capacity of radio communication systems with diversity in a Rayleigh fading environment," IEEE Journal on Selected Areas in Communications, vol. SAC-5. no. 5, pp. 871-878, June 1987.

[182] G. Foschini and J. Salz, "Digital communications over fading radio channels," Bell System Technical Journal, vol. 62, no. 2, pp. 429-456, February 1983.

[183] N. Amitay and J. Salz, "Linear equalization theory in digital data transmission over dually polarized fading radio channels," AT\&T Bell Laboratories Technical Journal, vol. 63, no. 10, pp. 2215-2259, December 1984.

[184] J. Salz, "Digital Transmission over cross-coupled linear channcls," AT\&T Technical Journal, vol. 64, no. 6, pp. 1147-1159. August 1985. 
[185] J. Salz and A. Wyner, "On data transmission over cross-coupled linear channels," unpublished work, June 1987.

[186] W. Rummler, "A new selective fading model: application to propagation data," Bell System Technical Journal, vol. 58, no. 5, pp. 1032-1071, May 1979.

[187] W. Rummler, "More on the multipath fading channel model," IEEE Transactions on Communications, vol. COM-29, no. 3, pp. 346-352, March 1981.

[188] M. Heath and L. Lopes, "A class of binary linear modulation techniques for personal communications systems," in Proceedings of the 1989 Global Telecommunications Conference, (Dallas, Tx.), pp. 1708-1713, December 1989.

[189] J. Chuang, "The effects of delay spread on 2-PSK, 4-PSK, 8-PSK and 16-QAM in a portable radio environment," IEEE Transactions on Vehicular Technology, vol. 38, no. 2 , pp. 43-45, May 1989.

[190] J. Chuang. "Comparison of coherent and differential detection of BPSK and QPSK in a quasi-static fading channel," in Proceedings of the 1988 IEEE International Conference on Communications, (Philadelphia, Pa.), pp. 749-755, June 1988.

[191] R. Gitlin, E. Ho, and J. Mazo, "Passband equalization of differentially phase-modulated data signals," Bell System Technical Journal, vol. 52, no. 2. February 1973.

[192] G. Turin. "The characteristic function of hermitian quadratic forms in complex nomal variables," Biometrika, pp. 199-201, June 1960.

[193] W. B. M. Schwartz and S. Stein, Communication Systems and Techniques. New York: McGraw-Hill, 1966.

[194] J. Wozencraft and 1. Jacobs, Principles of Communication Engineering. New York: John Wiley and Sons, 1965.

[195] S. Yoshida and F. Ikegami, "A comparison of multipath distortion characteristics among digital modulation techniques," IEEE Transactions on Vehicular Technology, vol. VT-34, no. 3, pp. 128-135, August 1985.

[196] M. Abdulrahman and D. Falconer, "Crosstalk suppression by decision feedback equalization on digital subscriber loops," in Proc. Canadian Conference on Electrical and Computer Engineering, (Ottawa, Canada), September 1990.

[197] M. Abdulrahman and D. Falconer, "Cyclostationary cmsstalk suppression by decision equalization on digital subscriber loops," submitted to IEEE Transactions on Communications.

[198] B. Petersen and D. Falconer, "Exploiting cyclostationary subscriber-loop interference by cqualization." in Proceedings of the 1990 IEEE Global Telecommunications Conference, (San Diego, Ca.), pp. 1156-1160, December 1990.

[199] B. Petersen and D. Falconer, "Minimum mean-square equalization in cyclostationary and stationary interference - analysis and subscriber-line calculations," To appear in IEEE Journal on Selected Areas in Communications, 1991. 
[200] J. Chuang, "Modeling and analysis of a digital portable communications channel with time delay spread," in Proceedings of the 1986 IEEE Vehicular Technology Conference, (Dailas, Tx.), pp. 246-251, May 1986.

[201] J. Smith, “A computer generated multipath fading simulation for mobilc radio," IEEE Transactions on Vehicular Technology, vol. VT-24, no. 3, pp. 39-40, August 1975.

[202] G. Arredondo and W. Chriss, "A multipath fading simulator for mobile radio," IEEE Transactions on Vehicular Technology, vol. VT-22, no. 4, pp. 241-244, November 1973.

[203] F. Glave and A. Rosenbaum, "An upper bound analysis for coherent phase-shift keying with cochannel, adjacent channel and intersymbol interference." IEEE Transactions on Communications, vol. COM-23, no. 6, pp. 600-612, June 1975.

[204] K. Yao and E. Biglieri. "A new approach for evaluating the error probability in the presence of intersymbol interference and additive Gaussian noise," IEEE Transactions on Information Theory, vol. IT-26, no. 7, pp. 454-464, July 1980.

[205] G. V. S. Benedetto and V.Castellani, "Combined effects of intersymbol, interchannel and cochannel interferences in M-ary CPSK systems," IEEE Transactions on Communications, vol. COM-2i, no. 9. pp. 997-1007, September 1973.

[206] G. V. S. Benedetto and A. Luvison, "Application of Gauss quadrature rules to digital communication problems," IEEE Transactions on Communications, vol. COM-21, no. 10 , pp. 1159-1165, October 1973.

[207] G. Golub and J. Welsch, "Calculation of Gauss quadrature rules," Math. Comput., vol. 23, pp. 221-230, 1969.

[208] W. Gautschi, "On the construction of Gaussian quadrature rules from modified moments," Math. Comput., vol. 24, pp. 245-260, April 1970.

[209] M. Meyers, "Computing the distribution of a random variable via gaussian quadrature rules," Bell System Technical Jourrnal, vol. 61, no. 9, pp. 2245-2261, November 1982.

[210] L. Hanzo, K. Wong, and R. Steele. "Efficient channel cxding and interleaving schemes for mobile radio communications," in Proceedings of the IEE Colloqium on Microcellular Mobile Radio, (London, U.K.), February 1989.

[211] S. Hanna, Private communication, June 1991.

[212] L. Chang and P. Porter, "Performance comparison of antenna diversity and slow frequency hopping for the TDMA portable radio channel," IEEE Transactions on Vehicular Technology, vol. 38, no. 4, pp. 222-229, November 1989.

[213] G. Stuber, L. Yin, E. Long, and K. Yang, "Outage control in digital cellular systems," IEEE Transactions on Vehicular Technology, vol. 40, no. 1, pp. 177-187, February 1991.

[214] D. Goodman and C. Sundberg, "The effect of channel coding on the efficiency of cellular mobile radio systems," IEEE Transactions on Communications, vol. COM-33, no. 3, pp. 288-291, March 1985. 
[215] C. Lin and J. Anderson, "M-algorithm decoding with path recovery for convolutional channel coding." in Proceedings of the IEEE 1986 Internationa' Conference on Communications, (Houston, Tx.), June 1986.

[216] R. Fano, "A heuristic discussion of probabilistic coding," IEEE Transactions on Information Theory, vol. IT-9, pp. 64-74, April 1963.

[217] F. Jelinek, "Fast sequential decoding algorithm using a stack," IBM Journal of Research and Development, vol. 13. pp. 675-685, November 1969.

[218] K. Zigangirov, "Some sequential decoding procedures," Probl. Peredach. Inform., vol. 2, pp. 13-25, 1966.

[219] L. Brandenburg and A. Wyner, "Capacity of the Gaussian channel with memory: the multivariate case," Bell System Technical Journal, vol. 53, no. 5, pp. 745-779, May 1974.

[220] M. Spiegel, Advanced Mathematics for Engineers and Scientists. New York: McGrawHill, 1971. 


\section{Appendix A \\ Recursive computation of partial fraction expansion coefficients}

Given

$$
F(s)=(P(s))^{d}=\prod_{i=1}^{\Lambda}\left(1+s \lambda_{i}\right)^{-n_{i} d}
$$

where the $\lambda_{i} \quad(i=1, \ldots \Lambda)$ are the distinct eigenvalues, each with multiplicity $n_{8}$ of the matrix $\overline{\text { q0oqoo }_{0}^{* T}} \mathbf{C}^{-1}$ (Chapter3, Sections 3.4 and 3.5), it is desired to find the coefficients $B_{i j}$ of the partial fraction expansion of $F(s)$, i.e.

$$
F(s)=\sum_{i=1}^{\lambda} \sum_{j=1}^{n_{i} d} \frac{B_{i j}}{\left(1+s \lambda_{i}\right)^{j}}
$$

A recursion formula for determining these coefficients has been developed by Monsen [128] for the special case where all eigenvalues have the same multiplicity $n_{i}=n$. It is now shown how this technique can be extended to the case where the eigenvalues have variable multiplicity.

If

$$
G_{j}(s)=\prod_{i=1(i \gtrless j)}^{\Lambda}\left(1+s \lambda_{i}\right)^{-n_{i} d}
$$

and

$$
G_{3}^{(k)}(s)=\frac{d^{k}}{d s^{k}} G_{3}(s)
$$

the differentiation of equation A.2 leads to

$$
B_{j,(n, d)-l}=\frac{1}{\lambda_{j}^{l} l !} G_{j}^{(k)}\left(-\frac{1}{\lambda_{j}}\right) \quad l=0.1 .2, \ldots .\left(n_{2} l-1\right)
$$

If equation A.3 is differentiated, one then obtains

$$
G_{j}^{(1)}(s)=G_{j}(s) Q_{j}(s)
$$

where

$$
Q,(s)=\sum_{i=1(i \gtrless J)}^{t o t} n_{i} d\left[-\frac{\lambda_{3}}{\left(1+s \lambda_{2}\right)}\right]
$$


Using the product rule for differentiation (as in [128]), $G_{j}^{(n+1)}(s)$ is seen to be a binomial expansion, i.e.

$$
G_{j}^{(n+1)}(s)=\sum_{m=1}^{n}\left(\begin{array}{c}
n \\
m
\end{array}\right) G_{j}^{(n-m)}(s) Q_{j}^{(m)}(s)
$$

where

$$
Q_{j}^{(m)}(s)=\frac{d}{d s^{m}} Q_{j}(s)=\sum_{i=1(i \geqslant j)}^{t o t}\left(n_{i} d\right) m !\left[-\frac{\lambda_{i}}{\left(1+s \lambda_{i}\right)^{m+1}}\right]
$$

The coefficient recursion is thus:

$$
\begin{aligned}
& B_{,,(n, d)}=G_{j}\left(-\frac{1}{\lambda_{j}}\right) \\
& \text { and } \\
& B_{j,(n, d-l)}=\frac{1}{l} \sum_{k=1}^{l} B_{j, n, d-l+k} \frac{Q_{j}^{(k-1)}\left(-\frac{1}{\lambda_{j}}\right)}{\lambda_{j}^{k}(k-1) !} \\
& \text { where } j=1,2, \ldots, \Lambda
\end{aligned}
$$




\section{Appendix B \\ Bit error rate bounds in flat Rayleigh fading}

This Appendix summarizes the bit error rate expressions for $\mathbf{M}^{\text {th }}$-order maximalratio combining of coherent BPSK and QPSK as well as binary DPSK in flat Rayleigh fading with no cochannel interference. It also gives the bit error rate bounds for BPSK and binary DPSK with convolutional coding and soft-decision Viterbi decoding under the assumption of ideal interleaving over a that Rayleigh fading channel (with no diversity and no interference). These expressions are obtained dirctly from [71, Chapter 7] or after some simple manipulations of expressions in [71, Chapter 7].

1. BER of $\mathbf{M}^{\text {th }}$-order maxinal-ratio combining for BPSK and binary DPSK in flat Rayleigh fading [71, Section 7.4]:

$$
P_{b}=\left(\frac{1-\mu}{2}\right) \sum_{i=0}^{M-1}\left(\begin{array}{c}
M-1+i \\
i
\end{array}\right)\left(\frac{1+\mu}{2}\right)^{2}
$$

where

$$
\mu= \begin{cases}\sqrt{\frac{\frac{E_{b}}{N_{1}}}{1+\frac{E_{b}}{N_{0}}}} & \text { BPSK } \\ \frac{\frac{E_{b}}{N_{4}}}{1+\frac{\frac{k}{6}_{6}}{N_{10}}} & \text { binary DPSK }\end{cases}
$$

2. BER of $M^{\text {th }}$-order maximal-ratio combining for QPSK in flat Rayleigh fading 171, Section 7.6]:

$$
P_{b}=\frac{1}{2}\left[1-\frac{\mu}{\sqrt{2-\mu^{2}}} \sum_{i=0}^{M-1}\left(\begin{array}{c}
2 i \\
i
\end{array}\right)\left(\frac{1-\mu^{2}}{4-2 \mu^{2}}\right)^{3}\right]
$$

where

$$
\mu=\sqrt{\frac{\frac{2 E_{b}}{N_{10}}}{1+\frac{2 E_{6}}{N_{11}}}}
$$


3. BER bound for BPSK and binary DPSK with convolutional coding of rate $\frac{k}{n}$ and soft-decision Viterbi decoding oyer a flat Rayleigh fading channel with ideal interleaving [71, Section 7.8]:

$$
P_{b}<\frac{1}{k} \sum_{d=d_{\text {jree }}}^{\infty} \beta_{d} P_{2}(d)
$$

where the code has free distance $d_{\text {free }}$, the $\beta_{d}$ 's ane obtained from the first derivative of the code's transfer function and where the pairwise error probabilities are given by

$$
P_{2}(d)=\left(\frac{1-\mu}{2}\right) \sum_{i=0}^{d-1}\left(\begin{array}{c}
d-1+i \\
i
\end{array}\right)\left(\frac{1+\mu}{2}\right)^{i}
$$

It should be noted that equations B.1 and B.6 are essentially identical as coding is just another form of diversity. In this sense, the parameter $\mu$ is given by

$$
\mu= \begin{cases}\sqrt{\frac{R_{c} \frac{E_{b}}{N_{i}}}{1+R_{c}}} & \text { BPSK } \\ \frac{R_{c} \frac{E_{b}}{N_{0}}}{1+R_{c} \frac{E_{b}}{N_{0}}} & \text { binary DPSK }\end{cases}
$$

Note that with M=1, equation B.1 (with BPSK) and equation B.3 both collapse to

$$
P_{b}=\frac{1}{2}\left[1-\sqrt{\frac{\frac{E_{b}}{N_{11}}}{1+\frac{E_{b}}{N_{11}}}}\right]
$$

i.e. BPSK and QPSK both perform identically in terms of BER over a fiat Rayleigh fading channel. This is shown in Figure B.1a where the degradation of binary DPSK with respect to BPSK and QPSK is found to be $3 \mathrm{~dB}$ at a BER of $10^{-3}$. The same is true with dual-order $(M=2)$ maximal-ratio combining; i.e. BPSK and QPSK also perform identically in terms of BER and also outperform binary DPSK by $3 \mathrm{~dB}$ at a BER of $10^{-3}$. On the other hand, the degradation of coded binary DPSK with respect to coded BPSK is found to be, in Figure B.1b, $3.3 \mathrm{~dB}$ at a BER of $10^{-3}$. 

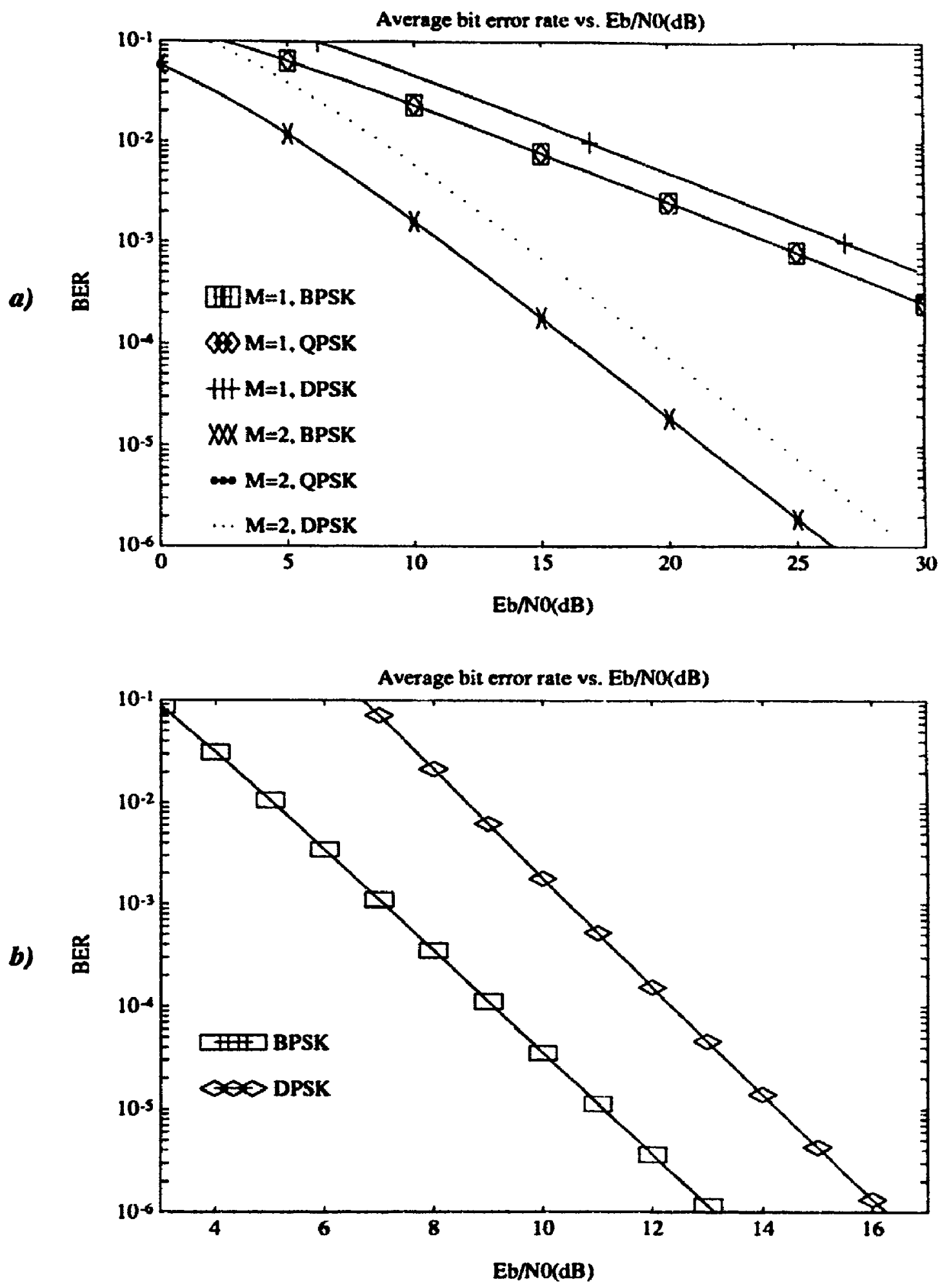

Figure B.1 Theoretical BER bounds from [71]. $N=0, L=1$, flat Rayleigh fading, time-rectangular pulse shape. a) Uncoded, $\mathbf{M}=$ \# of antennas b) Coded with ideal interleaving, $M=1$ (rate $1 / 2$ convolutional code, $d_{\text {free }}=5$, constraint length 3 ). 


\section{Appendix $\mathrm{C}$ Simulation documentation}

The following pages contain a few examples of the BOSS modules created for the simulations. The objective here is not to describe the entire simulation program but only to show how it is implemented with BOSS. Note that from the top-level system, there can be as much as five levels of complexity (i.e. modules within modules) before one finds only modules supplied by BOSS. Also shown is a sample set of results for the coded system with two receiver antennas. 


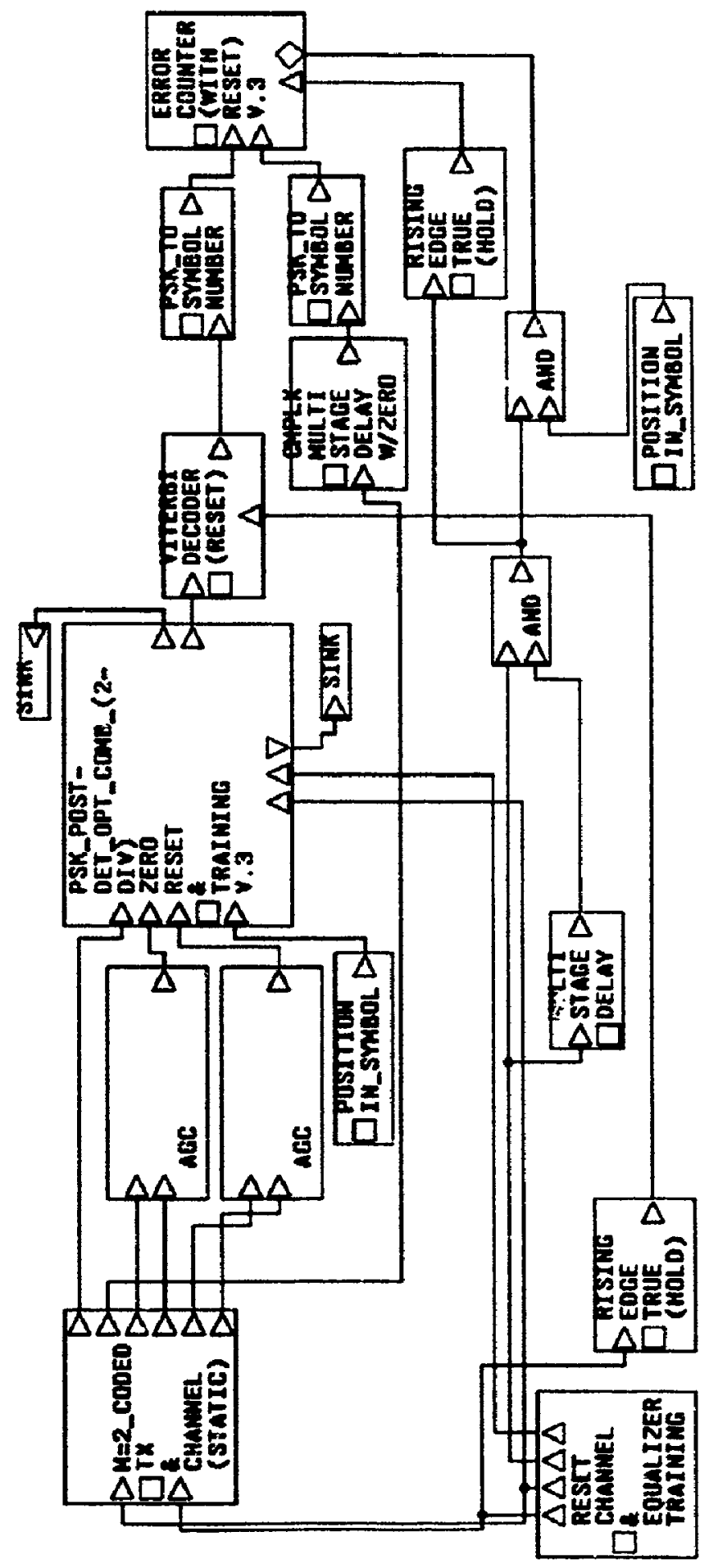

Figure C.1 BOSS module for the two receiver antenna system with coding. 


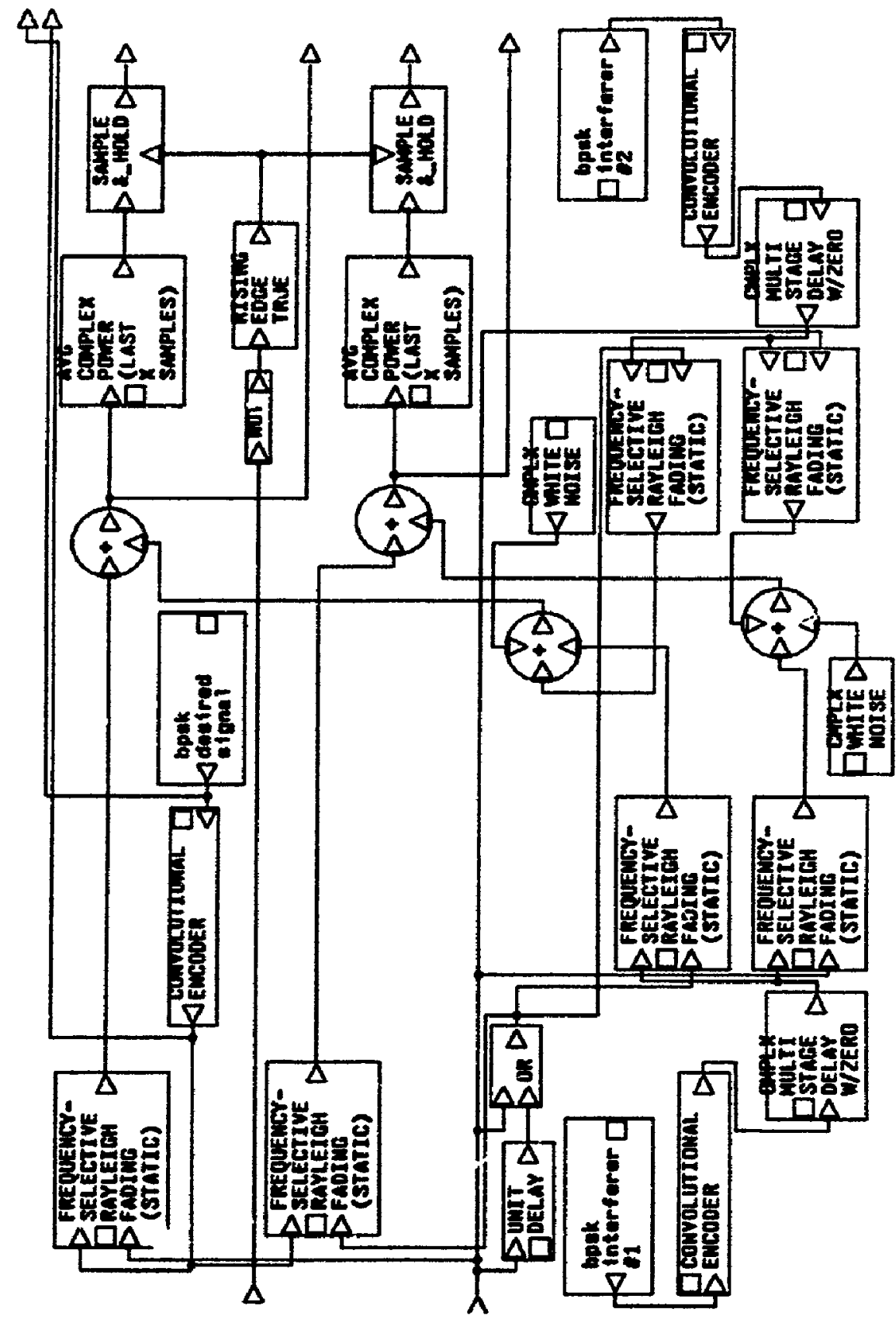

Figure C.2 BOSS module for the (coded) transmitter and channel. Corresponds to the "M=2_CODED Tx \& CHANNEL (STATIC)" module in Figure C.1. 


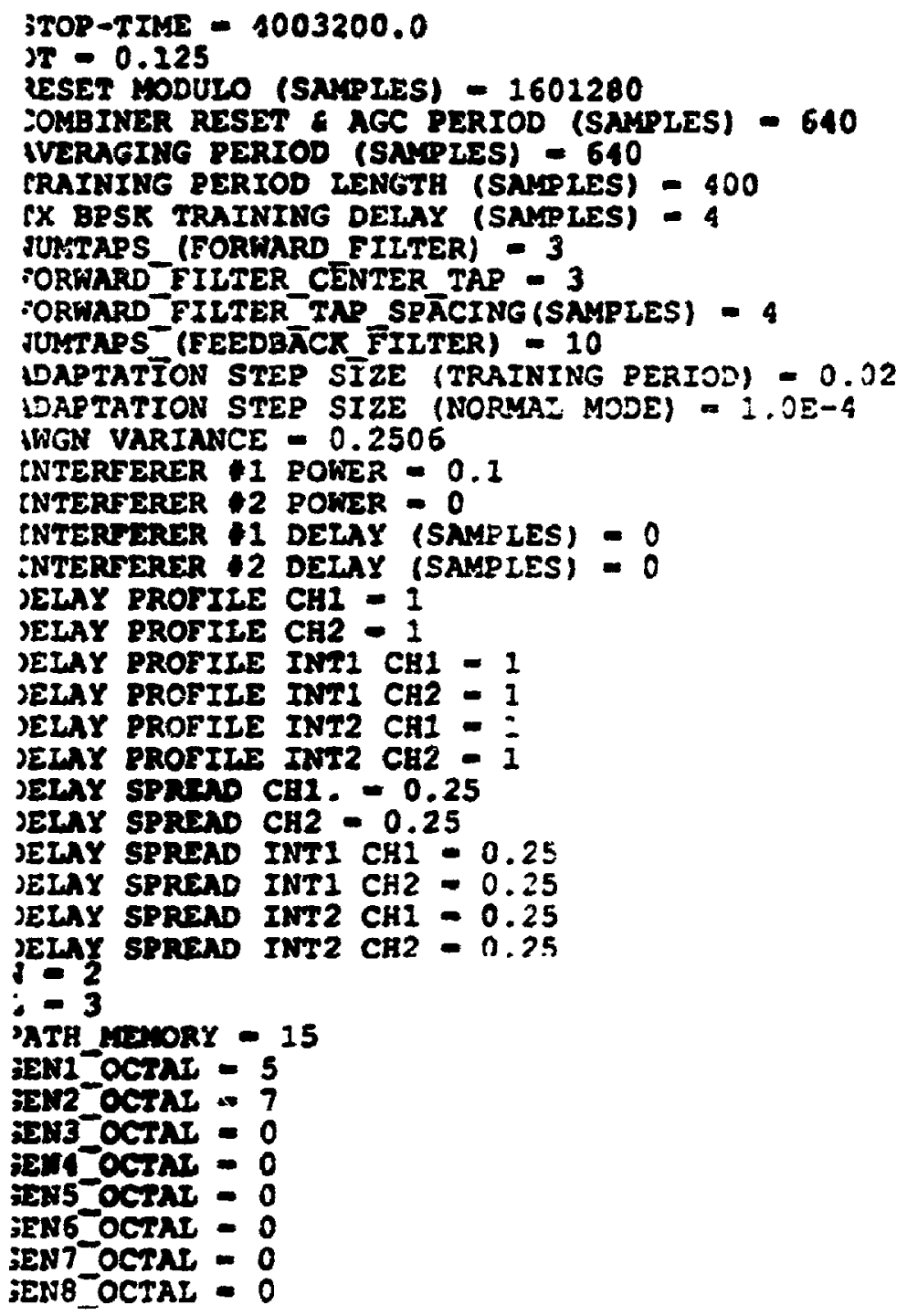

Figure $" .3$ Example of a parameter set for the system in figure C.1. 
cranist *

Totel thene of smols couneed o i 200690 ,

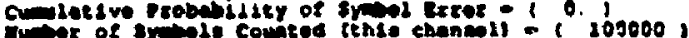

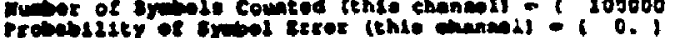

rropelsiter.

Totel L 2 es syote comated - 200000

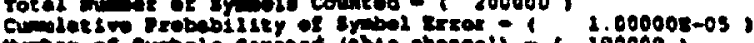

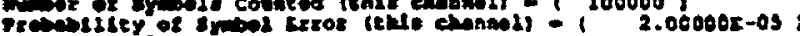

curnte

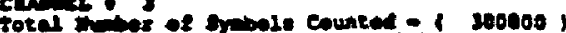

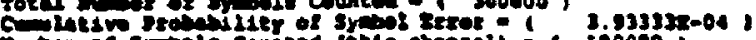

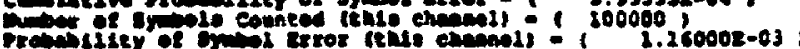

crinutes

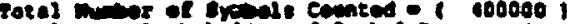

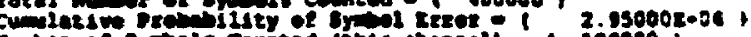

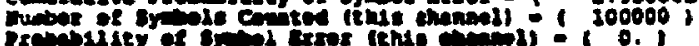

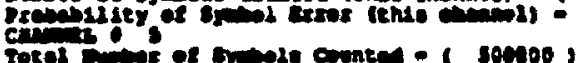

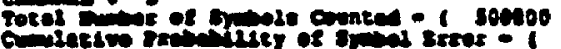

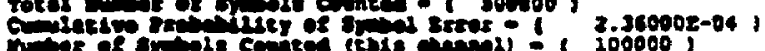

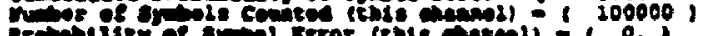

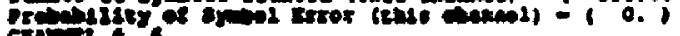

erint 4

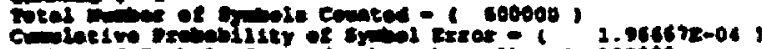

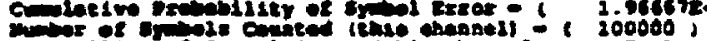

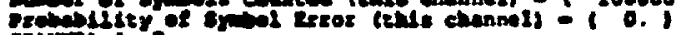

Crint 18

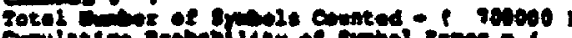

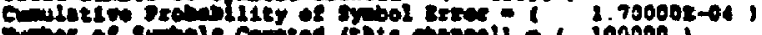

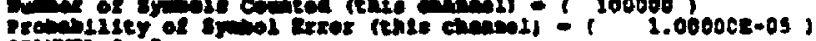

cinim

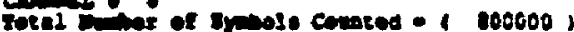

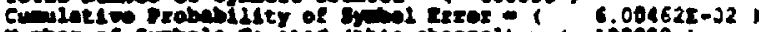

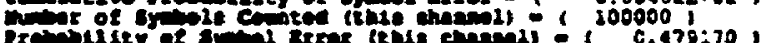

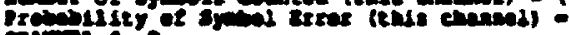

chens:

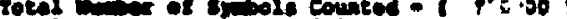

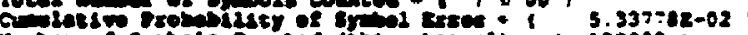

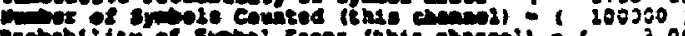

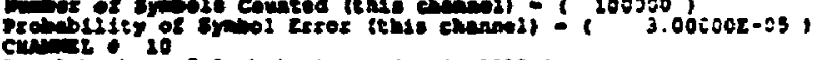

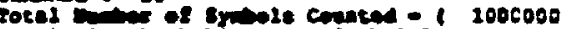

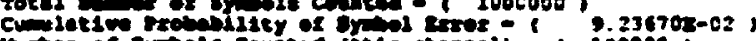

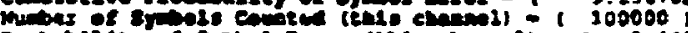

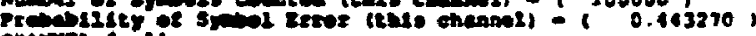

cunget 11

Sotel sume of symols counted - 1 11:0000

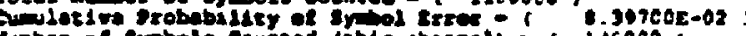

E jucoso

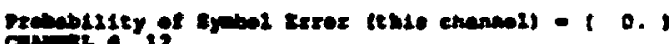

cin-3: 22

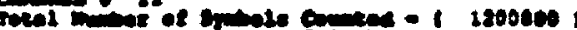

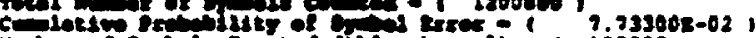

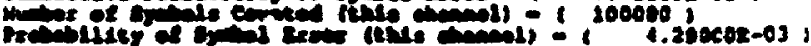

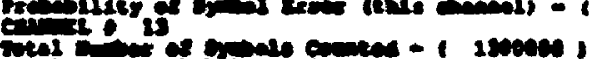

Geal

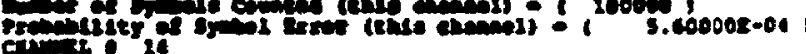

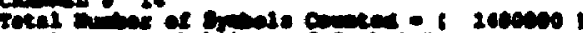

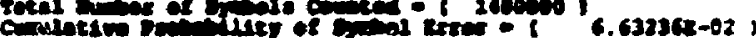

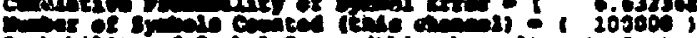

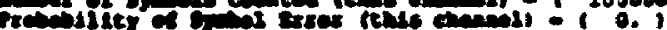

cinners

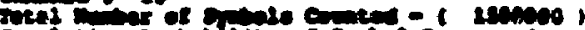

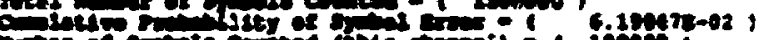

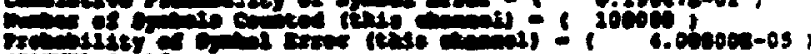

Ex+o 8

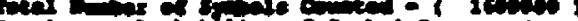

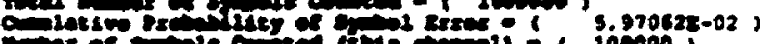

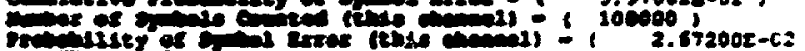

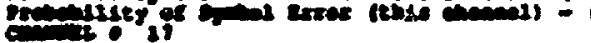

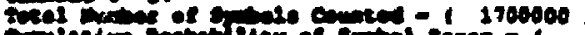

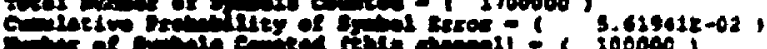

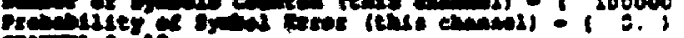

c. 18

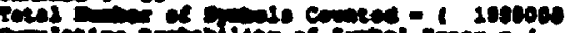

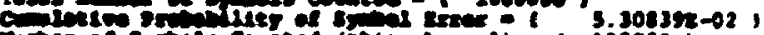

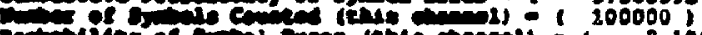

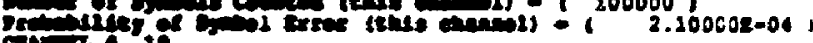

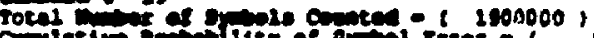

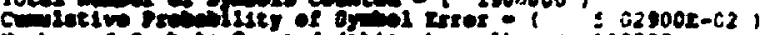

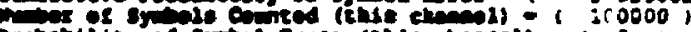

Protectutity of

cunce? 10

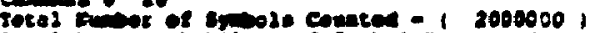

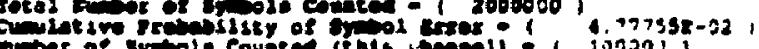

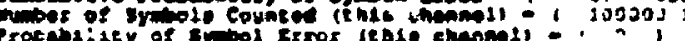

Figure C.t Example of a results set for the system in figure C.I. 


\section{Appendix D Capacity of indoor radio links}

As proposed in the suggestions for further research in Section 6.2 .4 of Chapter 6, it would be of interest to determine the average total capacity and average capacity per portable between $(N: 1)$ portables and a base station with $M$ antennas. This was performed in [18!] for the limiting case of a flat Rayleigh fading channel model, i.e. a single ray. It is now desired to obtain similar bounds for a realistic channel model such as the cluster model [60] employed in Chapter 5. This will provide information as to whether the large number of resolvable uncorrelated multipath echoes at high signaling rates (i.e. frequency-selective case) can improve capacity with respect to the single ray model (flat fading case).

First recall $[183,219]$ the capacity formula for a fixed ${ }^{84}$ scalar dispersive channel with frequency transfer characteristic $H(f)$, i.e. ${ }^{85}$

$$
C=\int_{W} \log _{2}\left[1+\frac{S}{N_{0}}|P(f)|^{2}|H(f)|^{2}\right] d f \quad \text { bit.s/scer. }
$$

where $S$ is the average signal power at the input to the transmitter filter $P(f)$ and $N_{0}$ is the double-sided power spectral density of the additive white Gaussian noise. The transmitter filter $P(f)$ is selected to maximize $C$ subject to an average transmitter power constraint.

For the multi-input, multi-output system shown in Figure D.1, the channel transfer and transmit filter characteristics are now respectively $M \times(V+1)$ and $(V+1) \times$ $(N+1)$ complex matrices. In this case, the channel capacity is $[219]^{86}$

$$
C=\sum_{n=0}^{N} \int_{W} \log _{2}\left[1+\frac{S_{n}}{N_{0}} x_{n}(f)\right] d f \quad \text { bits/scr }
$$

84 By averaging over capacities for a large number of different channel realizations, the "quasi-static capacity" approach considered herein does not evaluate the true channel capacity as it does not incorporate the effect of the time-varying channel's memory.

85 In the non-dispersive case considered in [181], equation D.1 collapses to equation 2.13 in Chapter 2, thus greatly simplifying the analysis.

86 Refer to equation 9 in Brandenburg and Wyner's paper [219]. 


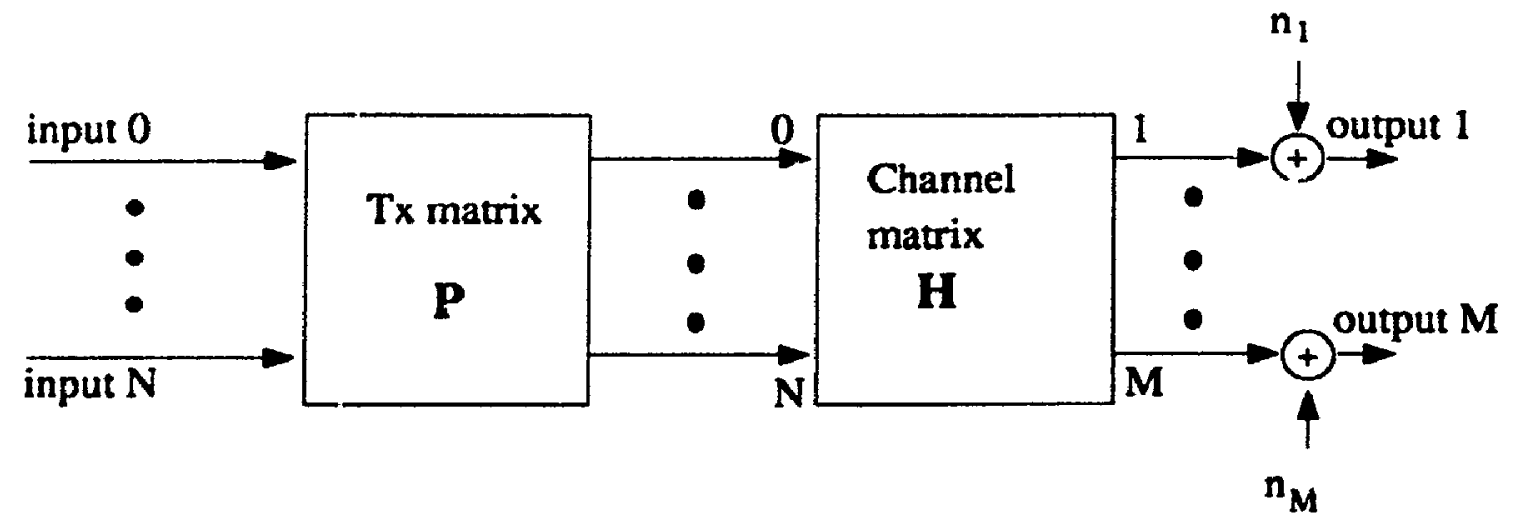

Figure D.1 Multi-input, multi-output system model for $(\mathrm{N}+1)$ portables and a base station with $M$ antennas. Channel matrix is $M \times(N+1)$ while transmitter matrix is $(N+1) \times(N+1)$.

where the $x_{n}(f), n=0,1, \ldots, N$ are the eigenvalues of the matrix ${ }^{87}$ $\mathbf{Y}(f)=\mathbf{P}^{* \mathbf{T}} \mathbf{H}^{* \mathbf{T}} \mathbf{H P}$.

As there is no communication between the portables, $\mathbf{P}(f)$ is a diagonal matrix with elements $P_{n}(f), \quad n=0,1, \ldots, N$. Hence, the total capacity of the system can now be written as

$$
\begin{aligned}
C & =\sum_{n=0}^{N} C_{n} \\
& =\sum_{n=0}^{N} \int_{W} \log _{2}\left[1+\frac{S_{n}}{N_{0}}\left|P_{n}(f)\right|^{2} \lambda_{n}(f)\right] d f \quad \text { bits } / \text { sec }
\end{aligned}
$$

where the $\lambda_{n}(f), n=0,1, \ldots, N$ are now the eigenvalues of $\Lambda(f)=\mathbf{H}^{* \mathbf{T}} \mathbf{H}$. In this way, the original multi-input, multi-output channel is represented as $(N+l)$ parallel channels, each with transfer characteristic $\sqrt{\lambda_{n}(f)}$. The transmit filter characteristics $P_{n}(f)$ must be chosen to maximize the corresponding $C_{n}$ subject to the constraint

$$
\int_{W}\left|P_{n}(f)\right|^{2} d f=1
$$

8) Diagonalizing this matrix via a unitary transformation $\boldsymbol{X}=\Omega^{* \boldsymbol{T}} \Delta \boldsymbol{\Omega}$ yields the diagonal matrix $\Delta$ whose clements are the eigenvalues of $\boldsymbol{Y}$. Recall that a unitary matrix $\boldsymbol{\Omega}$ is a square matrix whose inverse is equal to its conjugate. A unitary transformation will thus not atter the total average transmitted power [183]. 
i.e. each portable's transmit power is set to $S_{n}$.

In our application, the channels zee assumed to be strictly band-limited to a band $W=\frac{1}{T}$ where $T$ is the signaling rate. It is therefore convenient to normalize the capacity formula by the bandwidth W, i.e. $C_{W}=\underset{W}{W}$ bit.s/sfr/Hz. Substituting $z=f T=f$ in equation $D .3$ and dividing by $W$ then yields

$$
\begin{aligned}
C_{W} & =\sum_{n=0}^{N} C_{W^{\prime} n} \\
& =\sum_{n=0}^{N} \int_{-\frac{1}{2}}^{\frac{1}{2}} \log _{2}\left[1+\frac{S_{n}}{N_{0}}\left|P_{n}(z)\right|^{2} \lambda_{n}(z)\right] d z \quad \text { bit.s/.ser/Hz }
\end{aligned}
$$

where each $P_{n}(z)$ is selected to maximize $C_{W n}$ subject to

$$
\int_{-\frac{1}{2}}^{\frac{1}{2}}\left|P_{n}(z)\right|^{2} d z=1
$$

Applying the method of Lagrange multipliers and the calculus of variations [220] as in Amitay and Salz [183], the optimization of the $P_{n}(z)$ under the aforementioned constraint can thus be cast as finding (see Appendix E)

$$
M A X_{\left|P_{n}(z)\right|^{2} \geq 0}\left[\log _{2}\left(1+\frac{S_{n}}{N_{0}}\left|P_{n}(z)\right|^{2} \lambda_{n}(z)\right)-\lambda \frac{S_{n}}{N_{0}}\left|P_{n}(z)\right|^{2}\right]
$$

where $\lambda$ is a Lagrange multiplier. Setting the first derivative (with respect to $\left|P_{n}(z)\right|^{2}$ ) of equation D.7 to zero then yields (see Appendix E)

$$
\left|P_{n}(z)\right|^{2}=\operatorname{MAX}\left[0, \frac{N_{0}}{S_{n}}\left(\frac{1}{\lambda}-\frac{1}{\lambda_{n}(z)}\right)\right]
$$

The Lagrange multiplier is evaluated from the constraint of equation D.6, i.e.

$$
1=\int_{-\frac{1}{2}}^{\frac{1}{2}}\left[\operatorname{MAX}\left(0, \frac{N_{0}}{S_{n}}\left(\frac{1}{\lambda}-\frac{1}{\lambda_{n}(z)}\right)\right)\right] d z
$$


The determination of $\lambda$ and of the interval $Z_{n}$ where $\left|P_{n}(z)\right|^{2}>0$ can be accommodated by a simple iterative procedure ${ }^{88}$. From equations D.5 and D.8, the total normalized capacity is therefore

$$
\begin{aligned}
C_{W} & =\sum_{n=0}^{N} C_{W n} \\
& =\sum_{n=0}^{N} \int_{Z_{n}} \log _{2}\left(\frac{\lambda_{n}(z)}{\lambda}\right) d z
\end{aligned}
$$

The total average normalized capacity $\overline{C_{W}}$ and the average normalized capacity per portable ${ }^{89} \frac{\bar{C} \cdot i}{(i+1)}$ can thus be evaluated by generating a large number (e.g. 10000) of channel set realizations and averaging ${ }^{90}$ over all values of $C_{W}$ calculated by equation D.10. For the simple 9-tap channel model discussed earlier, the elements of the channel matrix ${ }^{91} H(f)$ are obtained via the Fourier transform of

$$
\begin{aligned}
h_{n i}(t) & =\sum_{r=0}^{8} \alpha_{r} \delta\left(t-\frac{r \sigma_{d}}{2}\right) \exp \left(-j \theta_{r}\right) \\
n & =0,1, \ldots, N \text { and } i=1, \ldots, M .
\end{aligned}
$$

i.e.

$$
\begin{aligned}
H_{n i}(f) & =\sum_{r=0}^{8} \alpha_{r} e^{-j\left(r x f \sigma_{d}+\theta_{r}\right)} \\
n & =0,1, \ldots, N \text { and } i=1, \ldots, M .
\end{aligned}
$$

where $\alpha_{r} e^{-j \theta_{r}}=x_{r}+j y_{r}, x_{r}$ and $y_{r}$ being independent zero-mean gaussian variates with a variance proportional to the delay profile (recall Chapter 4). The procedure is essentially similar for the cluster model [60] for which the number of paths is simply larger.

28 Refer to Appendix B in [183]. The procedure involves setting $\lambda^{(1)}=\operatorname{Min}_{|z| \leq t}\left[\lambda_{1}(z)\right]$ to ensure that $\left|P_{n}(z)\right|^{2}$ in equation $D .8$ is non-negative. One obtains the next value of $\lambda$, i.e. $\lambda^{(1)}$, by substituting $D .8$ in $D .6$ and solving for $\lambda$. If $\lambda^{(1)} \leq \lambda^{(0)}$, then $\lambda=\lambda^{(1)}$ and $Z_{n}$ is the whole interval $|z| \leq \frac{1}{2}$. If $\lambda^{(1)}>\lambda^{(0)}$, one finds $Z_{n}$ from $D .8$ and iterates for $\lambda$ until it ceases varying significantly (while updating $Z_{n}$ at each iteration).

89 Nove that the computational requirements ixcrease with the number of portables $(N+1)$.

90 As indicated earlier, this "quasi-static capacity" approach does not evaluate the true channel capacity as it does not incorporate the effect of the time-varying channel's memory.

91 Note that the elements of $\mathbf{H}(f)$ are all independent in this case as opposed to the dually polarized system of [183] where there is cross-coupled interference. 
Finally, it will be informative to compare these capacity bounds with the performance achievable by the uncoded and coded optimum baseband combiner. The various performance curves will yield the value of $\frac{E_{b}}{V_{1}}$ required to achieve a satisfactory average $\mathrm{BER}^{92}$ (e.g. $10^{-3}$ ) for a given normalized data rate (e.g. $1 \mathrm{bit} / \mathrm{sec} / \mathrm{Hz}$ and $2 \mathrm{bits} / \mathrm{sec} / \mathrm{Hz}$ for uncoded BPSK and QPSK respectively). However, the normalized capacity results are a function of the parameter $\frac{S}{N_{11}}$ as per equation D.5. The value of $\frac{f_{0}}{N_{11}}$ corresponding to a value of $C_{W}$ obtained from a given $\frac{S}{N_{11}}$, can be found simply by noting that

$$
S=C E_{b}=C_{W}, W E_{b}
$$

i.e.

$$
\frac{E_{b}}{N_{0}}=\left(\frac{S}{N_{0}}\right)\left(\frac{1}{W C_{W}}\right)
$$

The value of $W=\frac{1}{T}$ employed can be made to correspond to a data rate obtained from a given normalized ms delay spread and its typical absolute value.

92 One could also use outage rate as performance criteria to compare with the capacity bound. 


\section{Appendix E Lagrange multipliers and the calculus of variations}

The problem of selecting a $P_{n}(z)$ which maximizes

under the constraint

$$
\int_{-\frac{1}{2}}^{\frac{1}{2}} \log 2\left[1+\frac{S_{n}}{N_{0}}\left|P_{n}(z)\right|^{2} \lambda_{n}(z)\right] d z
$$

$$
\int_{-\frac{1}{2}}^{\frac{1}{2}}\left|P_{n}(z)\right|^{2} d z=1
$$

can be considered as part of a larger of class of problems where it is desired to find a curve $y(z)$ which makes a given integral ${ }^{93} \int_{z_{1}}^{z_{2}} F\left(z, y, y^{\prime}\right) d z$ a maximum or minimum but at the same time keeps the integral $\int_{z_{1}}^{z_{2}} G\left(z, y, y^{\prime}\right) d z$ equal to some constant. This type of problem in the calculus of variations is one which involves a constraint condition, namely that the latter integral is a constant. A special case of this is the problem of determining that curve having a given perimeter which encloses the largest area. Because of this special case, these problems are often referred to as isoperimetric problems [220].

Such problems can generally be solved by using the method of Lagrange multipliers. To do this, consider the integral

$$
\int_{z_{1}}^{z_{2}}\left[F\left(z, y, y^{\prime}\right)-\lambda G\left(z, y, y^{\prime}\right)\right] d z=\int_{z_{1}}^{z_{2}} H\left(z, y, y^{\prime}\right) d z
$$

where $\lambda$ is a Lagrange multiplier. This integral is an extremum if the Euler equation is satisfied, i.e. if [220]

$$
\frac{\partial}{\partial z}\left(\frac{\partial H}{\partial y^{\prime}}\right)-\frac{\partial H}{\partial y}=0
$$

Note that $y^{\prime}=\frac{\partial y}{\partial z}$. 
from which $\lambda$ and the required curve $y(z)$ can be found.

In our case, we thus form the function ${ }^{44}$

$$
\begin{aligned}
H & =\ln \left[1+\frac{S_{n}}{N_{0}}\left|P_{n}(z)\right|^{2} \lambda_{n}(z)\right]-\lambda \frac{S_{n}}{S_{0}}\left|P_{n}(z)\right|^{2} \\
& =\ln \left[1+\frac{S_{n}}{N_{n}} y \lambda_{n}(z)\right]-\lambda \frac{S_{n}}{N_{n}} y \\
& \text { where } y=\left|P_{n}(z)\right|^{2}
\end{aligned}
$$

Since $H\left(z, y, y^{\prime}\right)$ does not depend explicitly on $y^{\prime}$, the Euler equation is simply

$$
\frac{\partial H}{\partial y}=\frac{\partial}{\partial\left(\left|P_{n}(z)\right|^{2}\right)}\left\{\ln \left[1+\frac{S_{n}}{X_{10}}\left|P_{n}(z)\right|^{2} \lambda_{11}(z)\right]-\lambda \frac{S_{n}}{N_{0}}\left|P_{n}(z)\right|^{2}\right\}=0
$$

This yields

$$
\frac{\left(\frac{S_{u}}{I_{11}}\right) \lambda_{11}(z)}{\left(1+\frac{S_{p}}{X_{1}}\left|P_{u 1}(z)\right|^{2} \lambda_{11}(z)\right)}=\lambda\left(\frac{S_{u}}{S_{0}}\right)
$$

and therefore

$$
\left|P_{n}(z)\right|^{2}=\left(\frac{\lambda_{0}}{S_{n}}\right)\left(\frac{1}{\lambda}-\frac{1}{\lambda_{11}(z)}\right)
$$

which is seen to correspond to equation D.8 in Appendix D.

94 Note that maximizing $\ln (\ldots)$ is equivalent to maximizing $\ln (x, \ldots)=\left(\frac{1}{\ln |\ln | n \mid}\right)$. In addition. the constraint is taken to be $\frac{s_{n}}{x_{w 1}} \int_{-\frac{1}{t}}^{t}\left|P_{n}(z)\right|^{z} d z=\frac{i n}{i}$ instead of $\int_{-\frac{1}{t}}^{\frac{1}{3}}\left|P_{n}(z)\right|^{2} d z=1$. These scalar changes in no way affect the optimization process and are only intended to provide a more compact and elegant solution. 

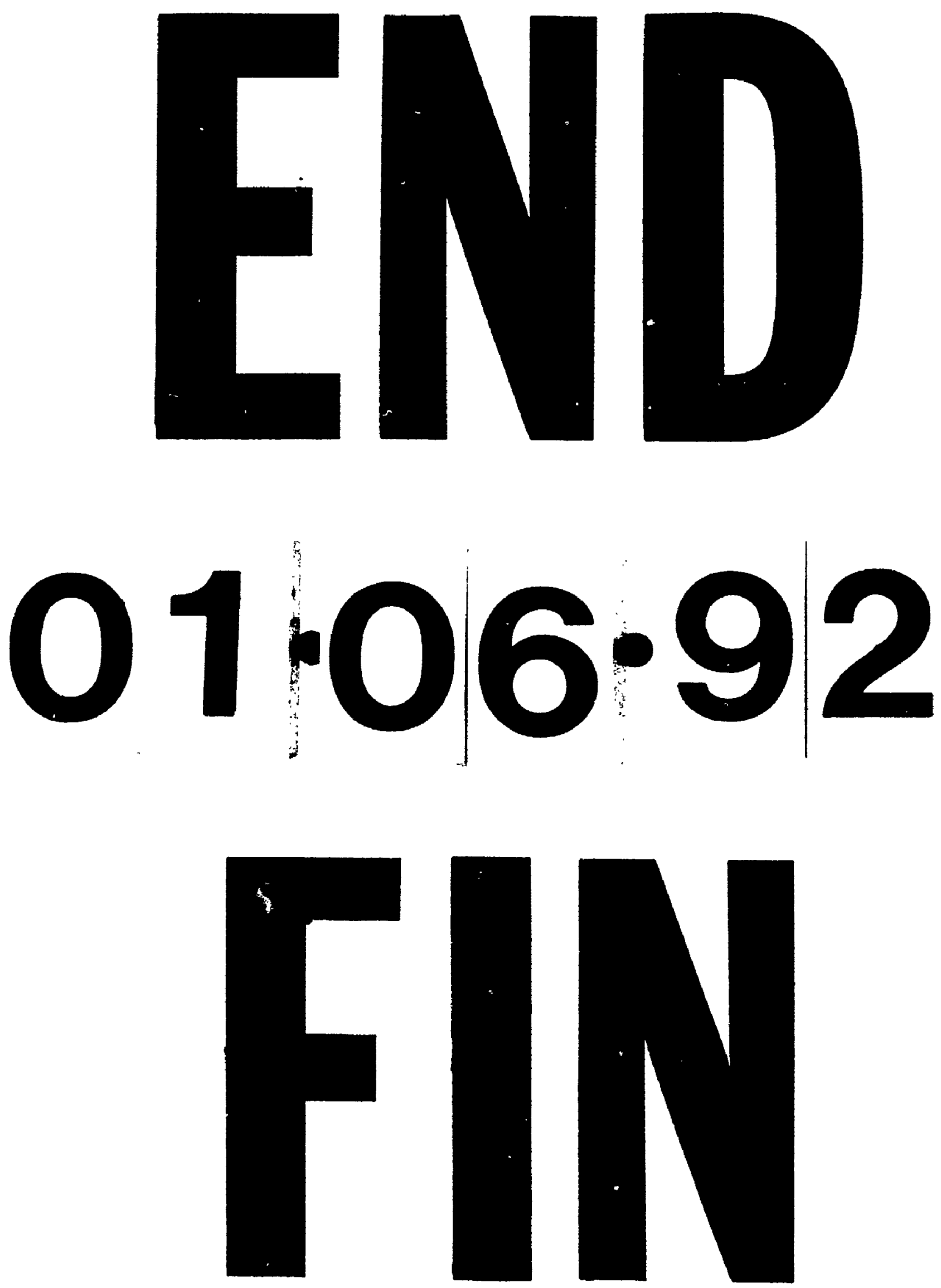